\title{
Cross-Coupling For Cross-Conjugation: Practical Synthesis and Diels-Alder Reactions of [3]Dendralenes
}

\author{
Tanya A. Bradford, ${ }^{\dagger}$ Alan D. Payne, ${ }^{\dagger}$ Anthony C. Willis, ${ }^{\dagger, \ddagger}$ \\ Michael N. Paddon-Row ${ }^{\star, \S}$ and Michael S. Sherburn ${ }^{\star,+, \pi}$
}

Research School of Chemistry, The Australian National University, Canberra

ACT 0200, Australia and School of Chemistry, The University of New South Wales, Sydney, NSW 2052, Australia.

sherburn@rsc.anu.edu.au (synthetic); m.paddonrow@unsw.edu.au (computational)

$\dagger$ The Australian National University

$\S$ The University of New South Wales

I ARC Centre of Excellence for Free Radical Chemistry and Biotechnology

\$ To whom correspondence should be addressed regarding X-ray crystal structures. E-mail: willis@ @rsc.anu.edu.au.

\section{Table of Contents}

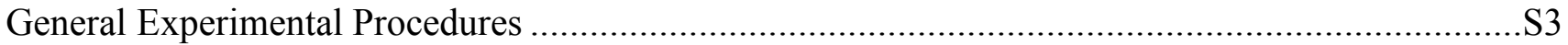

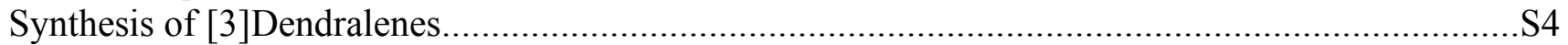

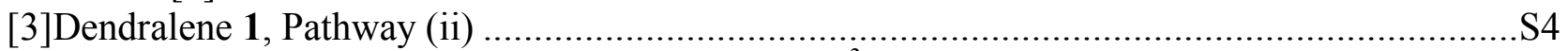

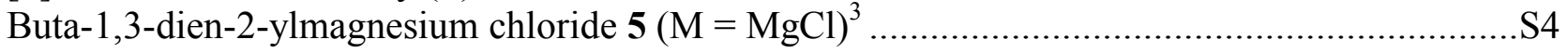

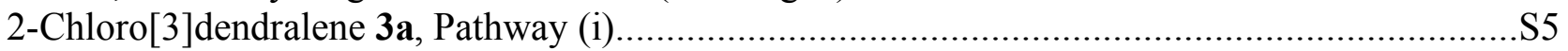

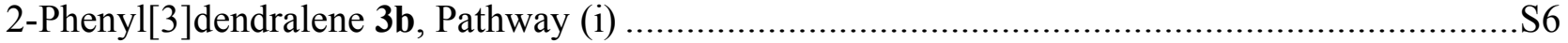

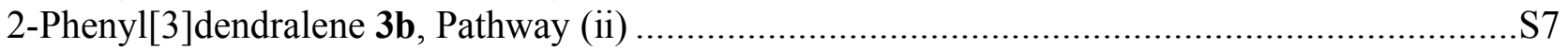

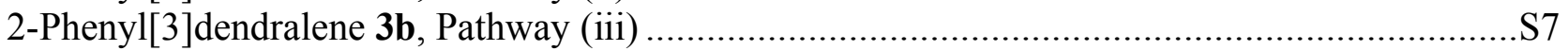

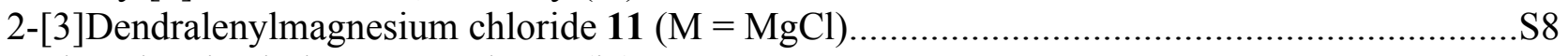

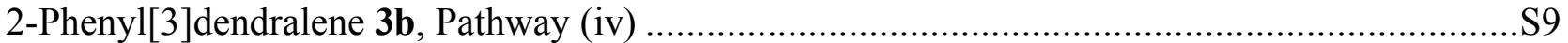

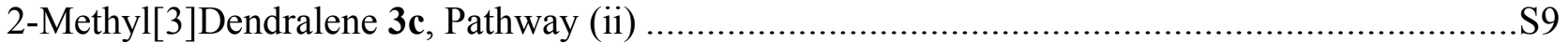

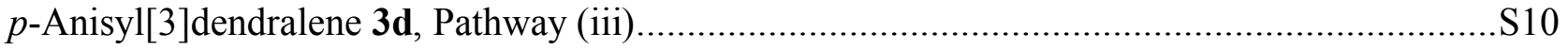

2-(4-Nitrophenyl)[3]dendralene 3e, Pathway (iv) ……......................................................... S11

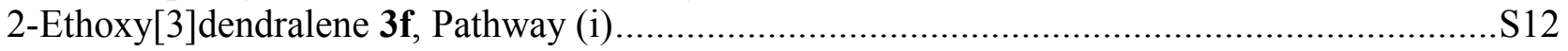

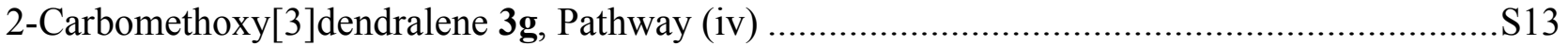

Diels-Alder Adducts of $\mathrm{N}$-methylmaleimide........................................................................S14

General Procedure for the preparation of the Diels-Alder Adducts with $N$-methylmaleimide .....S14

Diels-Alder reaction of 2-chloro[3]dendralene 3a with $N$-methylmaleimide ..............................S14

Diels-Alder reaction of 2-phenyl[3]dendralene 3b with $N$-methylmaleimide.............................S16

Diels-Alder reaction of 2-methyl[3]dendralene 3c with $N$-methylmaleimide.............................S17

Diels-Alder reaction with $p$-anisyl[3]dendralene 3d with $N$-methylmaleimide ...........................S19

Diels-Alder reaction of mono-adduct 16d with $N$-methylmaleimide .......................................S2

Diels-Alder reaction of 2-(4-nitrophenyl)[3]dendralene $3 \mathbf{e}$ with $N$-methylmaleimide..................S22 
Diels-Alder reaction of 2-ethoxy[3]dendralene $3 \mathbf{f}$ with $N$-methylmaleimide S23

Diels-Alder reaction of 2-carbomethoxy[3]dendralene $\mathbf{3 g}$ with $N$-methylmaleimide S25

Diels-Alder reaction of [3]dendralene 1 with $N$-methylmaleimide S26

Lewis Acid promoted Cycloaddition Reactions. S28

Treatment of 2-Phenyl[3]dendralene $\mathbf{3 b}$ with

$N$-Methylmaleimide-Ethylaluminium Dichloride Complex $(1: 1)$ S28

Treatment of 2-Phenyl[3]dendralene $\mathbf{3 b}$ with

$N$-Methylmaleimide-Ethylaluminium Dichloride Complex (1:2) S29

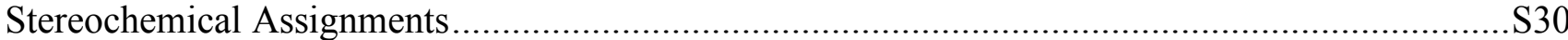

Stereochemical assignments of bis-adducts resulting from thermal and

Lewis acid promoted Diels-Alder reactions . $\mathrm{S} 30$

Stereochemical assignments of bis-adducts resulting from a

high pressure Diels-Alder reaction S35

References S39

Computational Details. S40

Anisotropic Displacement Ellipsoid Plots for 14a and 14d S47

${ }^{1} \mathrm{H}$ and ${ }^{13} \mathrm{C}$ NMR Spectra 


\section{General Experimental Procedures}

${ }^{1} \mathrm{H}$ NMR spectra were recorded at $298 \mathrm{~K}$ in $\mathrm{CDCl}_{3}$ using a $800 \mathrm{MHz}, 600 \mathrm{MHz}, 500 \mathrm{MHz}$ or $300 \mathrm{MHz}$ spectrometer. Residual chloroform ( $\delta 7.26 \mathrm{ppm})$ was used as an internal reference. ${ }^{13} \mathrm{C}$ NMR spectra were recorded at $298 \mathrm{~K}$ in $\mathrm{CDCl}_{3}$ at $125 \mathrm{MHz}$ or $75 \mathrm{MHz}$ with residual chloroform ( $\left.\delta 77.1 \mathrm{ppm}\right)$ as an internal reference. IR spectra were recorded as neat films on $\mathrm{NaCl}$ plates for oils or as $\mathrm{KBr}$ pellets for solid products. Low resolution mass spectra were recorded on an ion trap mass spectrometer using electron impact (EI) ionization mode at 40 or $70 \mathrm{eV}$. Low resolution electrospray ionization spectra were recorded on an ion trap mass spectrometer. High resolution mass spectra were recorded on a mass spectrometer operating at $70 \mathrm{eV}$. Melting points are uncorrected. Analytical TLC was performed with silica gel plates, precoated with silica gel 60 F254 $(0.2 \mathrm{~mm})$. Flash chromatography employed 230-400 mesh silica gel. Enantiomer ratios were determined by chiral GC (SGE CYDEX column, $25 \mathrm{~m} \times 0.22$ $\mathrm{mm}$ ID, $0.25 \mu$ film). Reactions were conducted under a positive pressure of dry argon or nitrogen. Benzene, diethyl ether, toluene and THF were dried over sodium wire and distilled from sodium benzophenone ketyl. Dichloromethane was distilled from calcium hydride. Commercially available chemicals were purified by standard procedures or used as purchased. 


\section{Synthesis of [3]Dendralenes}

\section{[3]Dendralene 1, Pathway (ii)}

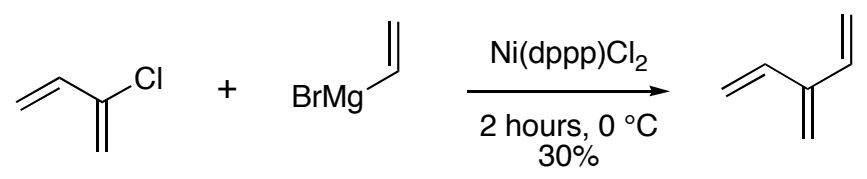

$\mathrm{Ni}(\mathrm{dppp}) \mathrm{Cl}_{2}$ (65 mg, $0.12 \mathrm{mmol}, 0.03$ equiv) was added to a stirred solution of vinyl magnesium bromide (4.70 mL of a $0.90 \mathrm{M}$ THF solution, $4.24 \mathrm{mmol}, 1.06$ equiv) and chloroprene ${ }^{1}$ (354 mg, 4 mmol, 1 equiv) under nitrogen at $0{ }^{\circ} \mathrm{C}$. After two hours the product was distilled $\left(0{ }^{\circ} \mathrm{C}, 10 \mathrm{mmHg}\right)$ from the reaction flask to a liquid nitrogen trap. [3]Dendralene $\mathbf{1}$ was isolated as a colourless THF solution (2.85 mL of a $0.42 \mathrm{M}$ solution, $96 \mathrm{mg}, 30 \%$ ). The compound had an identical ${ }^{1} \mathrm{H}$ n.m.r spectrum to that reported for $1 .{ }^{2}{ }^{1} \mathrm{H}$ NMR (THF solution: $\left.500 \mathrm{MHz}, \mathrm{CDCl}_{3}\right) \delta 6.44(2 \mathrm{H}, \mathrm{dd}, J=17.5$, $11.0 \mathrm{~Hz}), 5.40(2 \mathrm{H}, \mathrm{dd}, J=17.5,1.5 \mathrm{~Hz}), 5.15(2 \mathrm{H}, \mathrm{dd}, J=11.0,1.5 \mathrm{~Hz})$ and $5.14(1 \mathrm{H}, \mathrm{br} . \mathrm{s}) \mathrm{ppm}$.

\section{Buta-1,3-dien-2-ylmagnesium chloride $5(\mathrm{M}=\mathrm{MgCl})^{3}$}

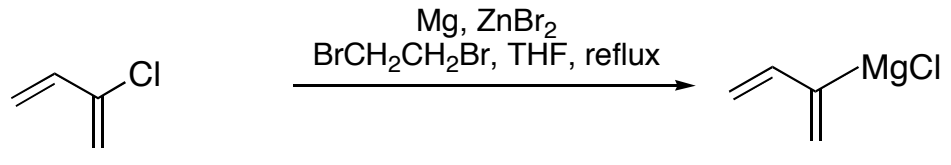

5

This compound was prepared by modification to the reported procedure. ${ }^{3}$ Oven-dried magnesium turnings (7.30 g, $300 \mathrm{mmol}, 1.59$ equiv) in anhydrous THF (50 mL) were stirred under nitrogen at room temperature. 1,2-Dibromoethane $(2.30 \mathrm{~mL}, 27 \mathrm{mmol}, 0.14$ equiv) was added portion wise (exotherm) followed by anhydrous zinc bromide (440 mg, $1.95 \mathrm{mmol}, 0.01 \mathrm{equiv})$. A solution of chloroprene $^{1}$ (16.7 g, $189 \mathrm{mmol}, 1$ equiv) and 1,2-dibromoethane (2.6 mL, $30 \mathrm{mmol}, 0.16$ equiv) in THF (100 mL) was added dropwise to the reaction mixture at such a rate to maintain reflux. The resulting solution was further heated to reflux for two hours. After cooling to room temperature, titration $^{4}$ of the dark green solution of buta-1,3-dien-2-ylmagnesium chloride 5 indicated a 1.08 M THF solution $(89 \%)$. 


\section{2-Chloro[3]dendralene 3a, Pathway (i)}

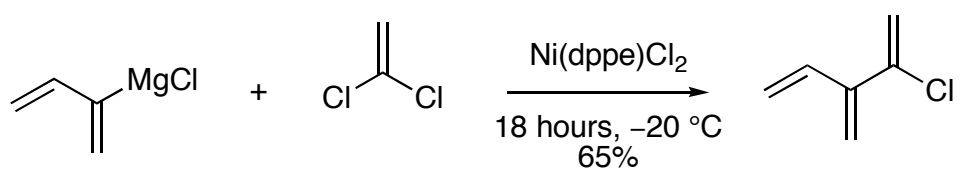

3a

$\mathrm{Ni}$ (dppe) $\mathrm{Cl}_{2}$ (319 mg, $0.52 \mathrm{mmol}, 0.01$ equiv) was added to a mixture of buta-1,3-dien-2-ylmagnesium chloride 5 (50 mL of a 1.0 M THF solution, $50 \mathrm{mmol}, 1$ equiv) and vinylidene chloride (20 mL, 250 mmol, 5 equiv) under nitrogen at $-20{ }^{\circ} \mathrm{C}$ and the resulting mixture was stirred at $-20{ }^{\circ} \mathrm{C}$ for 16 hours. The reaction mixture was poured into a stirred solution of $30-40{ }^{\circ} \mathrm{C}$ petrol: $\mathrm{H}_{2} \mathrm{O}(40: 60,200 \mathrm{~mL})$ and after 15 minutes the aqueous layer was removed. The organic layer was washed with $1 \mathrm{M} \mathrm{HCl}$ (aq.) $(50 \mathrm{~mL})$, sat. $\mathrm{NaHCO}_{3}$ (aq.) $(50 \mathrm{~mL})$, sat. brine $(50 \mathrm{~mL})$, dried over anhydrous magnesium sulfate, filtered and the solvent was removed under reduced pressure $\left(0{ }^{\circ} \mathrm{C}, 20 \mathrm{mmHg}\right)$ to yield 2chloro[3]dendralene 3a $(3.72 \mathrm{~g}, 65 \%)$ as a pale yellow oil. ${ }^{1} \mathrm{H}$ NMR $\left(300 \mathrm{MHz}, \mathrm{CDCl}_{3}\right) \delta 6.40(1 \mathrm{H}$, $\mathrm{dd}, J=17.4,10.8 \mathrm{~Hz}), 5.50(1 \mathrm{H}, \mathrm{d}, J=1.5 \mathrm{~Hz}), 5.47(1 \mathrm{H}, \mathrm{s}), 5.44(1 \mathrm{H}, \mathrm{d}, J=1.5 \mathrm{~Hz}), 5.36(1 \mathrm{H}, \mathrm{s})$, $5.30(1 \mathrm{H}, \mathrm{s})$ and $5.24(1 \mathrm{H}, \mathrm{dm}, J=10.8 \mathrm{~Hz}) \mathrm{ppm} ;{ }^{13} \mathrm{C} \mathrm{NMR}\left(75 \mathrm{MHz}, \mathrm{CDCl}_{3}\right) \delta 144.0(\mathrm{C}), 138.7(\mathrm{C})$, 134.4 (CH), $117.7\left(\mathrm{CH}_{2}\right), 117.6\left(\mathrm{CH}_{2}\right)$ and $114.9\left(\mathrm{CH}_{2}\right)$ ppm; IR (thin film): $v_{\max } 3087,1635$ and 1622 $\mathrm{cm}^{-1}$; UV/vis $\left(\mathrm{CH}_{3} \mathrm{CN}\right) \lambda_{\max } 215 \mathrm{~nm}(\varepsilon=11,000)$; EIMS $(70 \mathrm{eV}) \mathrm{m} / z(\%) 116\left(\mathrm{M}^{+},{ }^{37} \mathrm{Cl}, 6 \%\right), 114\left(\mathrm{M}^{+}\right.$, ${ }^{35} \mathrm{Cl}, 21$ ) and 79 (100); HRMS: calcd for $\mathrm{C}_{6} \mathrm{H}_{7}{ }^{35} \mathrm{Cl}\left[\mathrm{M}^{+}\right]$: 114.0236; found: 114.0235. 


\section{2-Phenyl[3]dendralene 3b, Pathway (i)}

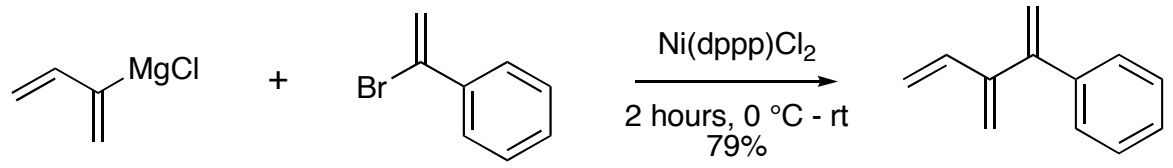

3b

$\mathrm{Ni}(\mathrm{dppp}) \mathrm{Cl}_{2}$ (445 mg, $0.82 \mathrm{mmol}, 0.03$ equiv) was added to a stirred solution of buta-1,3-dien-2ylmagnesium chloride 5 (20 mL of a 1.41 M THF solution, $28.2 \mathrm{mmol}, 1.03$ equiv) and $\alpha-$ bromostyrene (5 g, $27.3 \mathrm{mmol}, 1$ equiv) at $0{ }^{\circ} \mathrm{C}$ under nitrogen. After 30 minutes the reaction mixture was allowed to warm to room temperature and stirred for an additional one and a half hours. The reaction mixture was poured into a stirred solution of $30-40{ }^{\circ} \mathrm{C}$ petrol: $\mathrm{H}_{2} \mathrm{O}(40: 60,200 \mathrm{~mL})$ and the resulting mixture was stirred for 15 minutes. The organic layer was washed with $1 \mathrm{M} \mathrm{HCl}$ (aq.) (50 $\mathrm{mL})$, sat. $\mathrm{NaHCO}_{3}$ (aq.) $(50 \mathrm{~mL})$, sat. brine $(50 \mathrm{~mL})$, dried over anhydrous magnesium sulfate, filtered and the solvent was removed under reduced pressure $\left(0{ }^{\circ} \mathrm{C}, 20 \mathrm{mmHg}\right)$ to yield 2-phenyl[3]dendralene 3b $(3.38 \mathrm{~g}, 79 \%)$ as a yellow oil. ${ }^{1} \mathrm{H}$ NMR $\left(300 \mathrm{MHz}, \mathrm{CDCl}_{3}\right) \delta 7.26(5 \mathrm{H}, \mathrm{m}), 6.36(1 \mathrm{H}, \mathrm{dd}, J=17.0$, $10.5 \mathrm{~Hz}), 5.45(1 \mathrm{H}, \mathrm{s})$ and 5.11, $5.45(5 \mathrm{H}, \mathrm{m} ; 1 \mathrm{H}, \mathrm{s}) \mathrm{ppm} ;{ }^{13} \mathrm{C} \mathrm{NMR}\left(75 \mathrm{MHz}, \mathrm{CDCl}_{3}\right) \delta 148.3(\mathrm{C})$, $146.6(\mathrm{C}), 140.0(\mathrm{C}), 137.5(\mathrm{CH}), 128.3(\mathrm{CH}), 127.6(\mathrm{CH}), 126.8(\mathrm{CH}), 118.4\left(\mathrm{CH}_{2}\right) 117.7\left(\mathrm{CH}_{2}\right)$ and $114.9\left(\mathrm{CH}_{2}\right)$ ppm; IR (thin film): $v_{\max } 3087,3026,3007,1594$ and $1494 \mathrm{~cm}^{-1}$; UV/vis $\left(\mathrm{CH}_{3} \mathrm{CN}\right) \lambda_{\max }$ $201 \mathrm{~nm}(\varepsilon=28,000)$; EIMS (70 eV) m/z (\%) $156\left(\mathrm{M}^{+}, 40 \%\right), 129$ (54) and 105 (100); HRMS: calcd for $\mathrm{C}_{12} \mathrm{H}_{12}\left[\mathrm{M}^{+}\right]:$156.0939; found: 156.0938 . 


\section{2-Phenyl[3]dendralene 3b, Pathway (ii)}
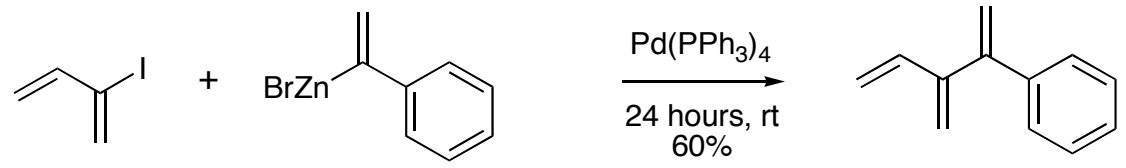

$3 \mathbf{b}$

A solution of anhydrous zinc bromide (480 mg, $2.13 \mathrm{mmol}, 3.45$ equiv) in anhydrous THF (1 mL) was cooled to $0{ }^{\circ} \mathrm{C}$ and $\alpha$-styrylmagnesium bromide ${ }^{5}$ (4.50 mL of a 0.44 M THF solution, $2.00 \mathrm{mmol}, 3.24$ equiv) was added dropwise. The mixture was stirred at $0{ }^{\circ} \mathrm{C}$ for 30 minutes and iodoprene ${ }^{6}(111 \mathrm{mg}$, $0.617 \mathrm{mmol}, 1$ equiv) and tetrakis(triphenylphophine)palladium(0) (42 mg, $0.04 \mathrm{mmol}, 0.06$ equiv) were added. The mixture was stirred at room temperature for 24 hours then diluted in $30-40{ }^{\circ} \mathrm{C}$ petrol $(50 \mathrm{~mL})$ and poured into water $(50 \mathrm{~mL})$. The organic layer was washed with $1 \mathrm{M} \mathrm{HCl}(\mathrm{aq}).(50 \mathrm{~mL})$ and sat. brine $(50 \mathrm{~mL})$, dried over anhydrous magnesium sulfate and concentrated under reduced pressure $\left(0{ }^{\circ} \mathrm{C}, 30 \mathrm{mmHg}\right)$. The resulting oil was subjected to radial chromatography $\left(30-40{ }^{\circ} \mathrm{C}\right.$ petrol) to give 2-phenyl[3]dendralene $\mathbf{3 b}$ as a colourless oil $(58 \mathrm{mg}, 60 \%)$.

\section{2-Phenyl[3]dendralene 3b, Pathway (iii)}
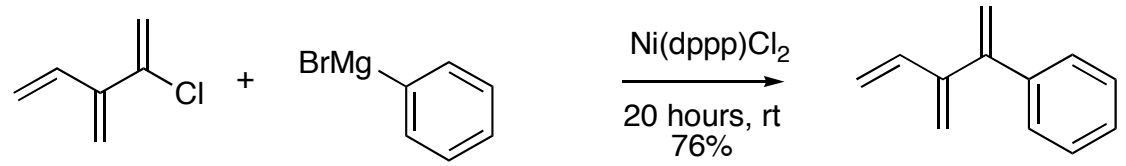

$3 \mathbf{b}$

Bromobenzene $(4.00 \mathrm{~mL}, 5.00 \mathrm{mmol}, 1.25$ equiv) was added in portions to a suspension of magnesium turnings (2.50 g, $103 \mathrm{mmol}, 25.8$ equiv) in anhydrous THF (50 mL). Once the reaction had subsided, the reaction mixture was heated under reflux for 30 minutes and then allowed to cool to room temperature. 2-Chloro[3]dendralene 3a (4.58 g, $4.00 \mathrm{mmol}, 1$ equiv), triphenylphosphine (200 mg, 0.76 mmol, 0.19 equiv) and $\mathrm{Ni}(\mathrm{dppp}) \mathrm{Cl}_{2}(200 \mathrm{mg}, 0.37 \mathrm{mmol}, 0.09$ equiv) were added successively to the reaction mixture. An exotherm was noted, following addition, and the mixture was stirred for 18 hours then poured into sat. brine $(200 \mathrm{~mL})$ and $30-40{ }^{\circ} \mathrm{C}$ petrol $(150 \mathrm{~mL})$ and stirred for 20 minutes. The organic layer was dried over anhydrous magnesium sulfate and concentrated under reduced pressure $(0$ 
${ }^{\circ} \mathrm{C}, 30 \mathrm{mmHg}$ ) to give an oil which was subjected to flash chromatography. Elution with $30-40{ }^{\circ} \mathrm{C}$ petrol gave 2-phenyl[3]dendralene $\mathbf{3 b}$ as a colourless oil $(4.75 \mathrm{~g}, 76 \%)$.

\section{2-[3]Dendralenylmagnesium chloride $11(\mathrm{M}=\mathrm{MgCl})$}

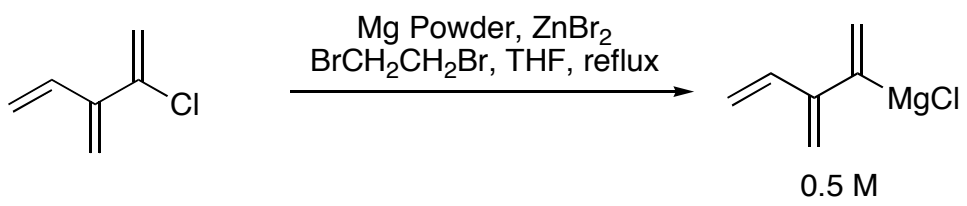

11

A mixture of magnesium powder (1.8 g, $74 \mathrm{mmol}, 2.1$ equiv) in anhydrous THF (30 ml) was placed in an oven-dried $250 \mathrm{ml}$ round bottom flask and 1,2-dibromoethane $(0.5 \mathrm{ml})$ was added in two portions. Once the reaction had subsided, anhydrous zinc bromide $(0.18 \mathrm{~g}, 8.0 \mathrm{mmol}, 0.3 \mathrm{equiv})$ was added and the mixture was heated to reflux for 20 minutes. Then a solution of 2-chloro[3]dendralene 3a (4.0 g, 35 mmol, 1 equiv) and 1,2-dibromoethane $(1 \mathrm{ml})$ in anhydrous THF $(35 \mathrm{ml})$ was added at such a rate as to maintain a vigorous reflux. After the addition was complete, the reaction was heated under reflux for a further 30 minutes. Formation of the Grignard reagent 11 was confirmed by the formation of [3]dendralene 1 upon hydrolysis (with $\mathrm{H}_{2} \mathrm{O}$ and $\mathrm{D}_{2} \mathrm{O}$ ) and by titration (Gilman double titration ca. 95\%). (Note: A concentration of $>0.5 \mathrm{M}$ leads to significant dimerisation products.) 


\section{2-Phenyl[3]dendralene 3b, Pathway (iv)}
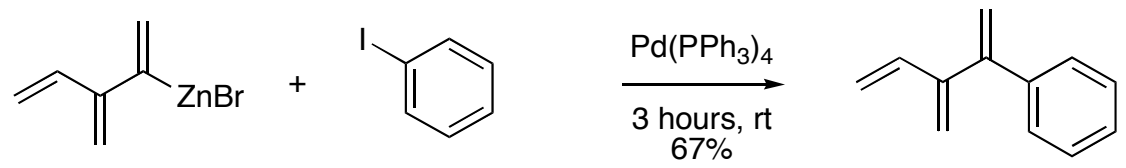

3b

2-[3]Dendralenylmagnesium chloride 11 (8.0 mL of a 0.5 M THF solution, $4.0 \mathrm{mmol}$, 1.6 equiv) was added dropwise to a stirred solution of anhydrous zinc bromide ( $0.9 \mathrm{~g}, 4.0 \mathrm{mmol}, 1.6 \mathrm{guiv})$ in anhydrous $\mathrm{THF}(2 \mathrm{ml})$ at $0{ }^{\circ} \mathrm{C}$ under nitrogen. The reaction mixture was stirred at room temperature for 20 minutes, then iodobenzene $(510 \mathrm{mg}, 2.50 \mathrm{mmol}, 1$ equiv) was added followed by tetrakis(triphenylphosphine)palladium(0) (96 mg, $0.08 \mathrm{mmol}, 0.03 \mathrm{equiv}$ ) and the reaction mixture was stirred for a further three hours. The reaction mixture was poured into water $(50 \mathrm{~mL})$ and extracted with $30-40{ }^{\circ} \mathrm{C}$ petrol $(50 \mathrm{~mL})$. The organic layer was dried over anhydrous magnesium sulphate and concentrated under reduced pressure $\left(0^{\circ} \mathrm{C}, 30 \mathrm{mmHg}\right)$ to give an oil which was subjected to flash chromatography. Elution with $30-40{ }^{\circ} \mathrm{C}$ petrol gave 2-phenyl[3]dendralene $3 \mathbf{b}$ as a colourless oil (264 $\mathrm{mg}, 67 \%)$.

\section{2-Methyl[3]Dendralene 3c, Pathway (ii)}

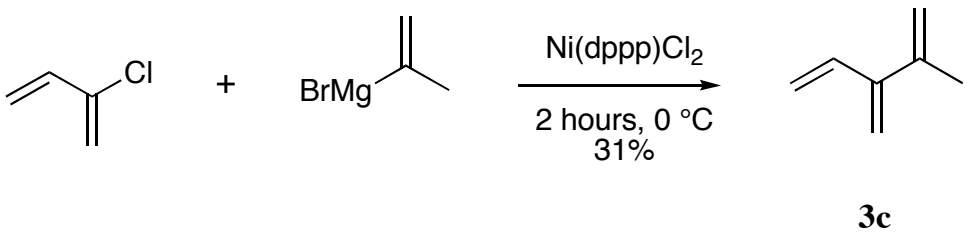

$\mathrm{Ni}(\mathrm{dppp}) \mathrm{Cl}_{2}$ (37 mg, $0.07 \mathrm{mmol}, 0.03$ equiv) was added to a solution of isopropenyl magnesium bromide (5.42 mL of a $0.5 \mathrm{M}$ THF solution, $2.71 \mathrm{mmol}, 1.2$ equiv) and chloroprene ${ }^{1}$ (200 mg, 2.26 mmol, 1 equiv) under nitrogen at $0{ }^{\circ} \mathrm{C}$ and the resulting mixture was stirred for two hours. The reaction mixture was poured into a stirred solution of $30-40{ }^{\circ} \mathrm{C}$ petrol: $\mathrm{H}_{2} \mathrm{O}(40: 60,50 \mathrm{~mL})$ and the resulting mixture was stirred for 15 minutes. The organic layer was washed with $1 \mathrm{M} \mathrm{HCl}$ (aq.) (20 $\mathrm{mL})$, sat. $\mathrm{NaHCO}_{3}$ (aq.) $(20 \mathrm{~mL})$, sat. brine $(20 \mathrm{~mL})$, dried over anhydrous magnesium sulfate and filtered to yield a solution of 2-methyl[3]dendralene 3c as a pale yellow solution in $30-40{ }^{\circ} \mathrm{C}$ petrol (34.5 mL of a $0.02 \mathrm{M}$ solution, $65 \mathrm{mg}, 31 \%$ ). The compound had an identical ${ }^{1} \mathrm{H}$ n.m.r spectrum to that 
reported for $2 .{ }^{7} \mathrm{H}$ NMR $\left(30-40{ }^{\circ} \mathrm{C}\right.$ petrol/THF solution: $\left.300 \mathrm{MHz}, \mathrm{CDCl}_{3}\right) \delta 6.48(1 \mathrm{H}, \mathrm{dd}, J=17.5$, $11.0 \mathrm{~Hz}), 5.37(1 \mathrm{H}$, br. d, $J=17.5 \mathrm{~Hz}), 5.13(1 \mathrm{H}$, br. d, $J=11.0 \mathrm{~Hz}), 5.12(2 \mathrm{H}$, br. d, $J=48.5 \mathrm{~Hz})$, $5.04(2 \mathrm{H}$, br. s) and $1.93(3 \mathrm{H}, \mathrm{s}) \mathrm{ppm}$.

\section{p-Anisyl[3]dendralene 3d, Pathway (iii)}

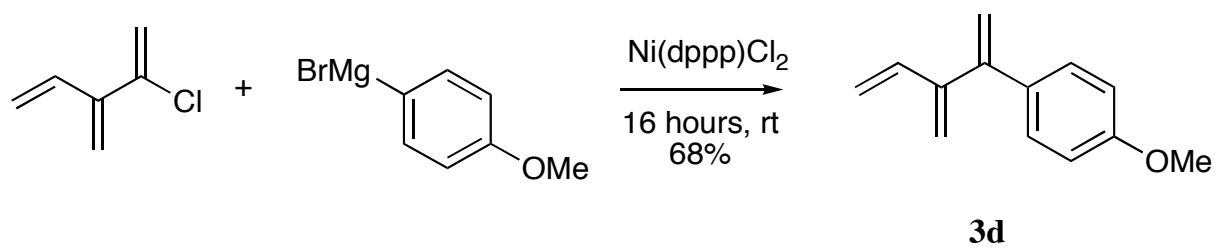

To a stirred solution of magnesium turnings ( $2.0 \mathrm{~g}, 82 \mathrm{mmol}, 8.2$ equiv) in anhydrous THF (20 mL), 1,2-dibromoethane $(1 \mathrm{~mL})$ was added in portions. After the reaction had subsided, the reaction mixture was placed in a water bath (rt) and $p$-bromoanisole $(2.5 \mathrm{~mL}, 20 \mathrm{mmol}, 2.0$ equiv) was added in 5 portions, and the reaction was stirred at room temperature for a further one hour. 2Chloro[3]dendralene 3a (1.15 g, $10 \mathrm{mmol}, 1.0$ equiv) was added followed by $\mathrm{Ni}(\mathrm{dppp}) \mathrm{Cl}_{2}$ (150 mg, $0.28 \mathrm{mmol}, 0.03$ equiv) and the mixture was stirred at room temperature for 18 hours. The reaction mixture was poured into water $(150 \mathrm{~mL})$ and $30-40{ }^{\circ} \mathrm{C}$ petrol $(100 \mathrm{~mL})$ and stirred for 15 minutes. 1 $\mathrm{M} \mathrm{HCl}$ (aq.) (100 mL) was added to disperse the emulsion. The organic layer was washed with water $(100 \mathrm{~mL})$ and dried over anhydrous magnesium sulphate and concentrated under reduced pressure $(0$ $\left.{ }^{\circ} \mathrm{C}, 30 \mathrm{mmHg}\right)$. The resulting oil was subjected to flash chromatography $\left(0-25 \% \mathrm{CHCl}_{3} / 40-60{ }^{\circ} \mathrm{C}\right.$ petrol) to give $p$-anisyl[3]dendralene $\mathbf{3 d}$ as a colourless oil (1.05 g, 68\%). Rf $0.14\left(25: 75 \mathrm{CHCl}_{3}: 40-\right.$ $60{ }^{\circ} \mathrm{C}$ petrol); ${ }^{1} \mathrm{H}$ NMR $\left(300 \mathrm{MHz}, \mathrm{CDCl}_{3}\right) \delta 7.32-7.38(2 \mathrm{H}, \mathrm{m}), 6.81-6.88(2 \mathrm{H}, \mathrm{m}), 6.45(1 \mathrm{H}, \mathrm{dd}, J=$ 16.9, $11.1 \mathrm{~Hz}), 5.45(1 \mathrm{H}, \mathrm{d}, J=1.6 \mathrm{~Hz}), 5.31(1 \mathrm{H}, \mathrm{d}, J=2.2 \mathrm{~Hz}), 5.19(1 \mathrm{H}, \mathrm{m}), 5.15(1 \mathrm{H}, \mathrm{d}, J=1.6$ $\mathrm{Hz}), 5.05(1 \mathrm{H}, \mathrm{d}, J=16.9 \mathrm{~Hz}), 5.04(1 \mathrm{H}, \mathrm{d}, J=11.1 \mathrm{~Hz})$ and $3.81(3 \mathrm{H}, \mathrm{s}) \mathrm{ppm} ;{ }^{13} \mathrm{C}$ NMR $(75 \mathrm{MHz}$, $\left.\mathrm{CDCl}_{3}\right)$ \& $159.1(\mathrm{C}), 148.4(\mathrm{C}), 147.3(\mathrm{C}), 137.3(\mathrm{CH}), 132.4(\mathrm{C}), 127.8(\mathrm{CH}), 118.2\left(\mathrm{CH}_{2}\right), 117.4$ $\left(\mathrm{CH}_{2}\right), 113.5(\mathrm{CH}), 113.1\left(\mathrm{CH}_{2}\right), 55.2\left(\mathrm{CH}_{3}\right) \mathrm{ppm}$; IR (thin film): $v_{\max } 2934$ and $1607 \mathrm{~cm}^{-1} ; \mathrm{UV} / \mathrm{vis}$ (hexane) $\lambda_{\max } 203 \mathrm{~nm}(\varepsilon=117,000), 256 \mathrm{~nm}(\varepsilon=54,000)$. EIMS $(70 \mathrm{eV}) \mathrm{m} / z(\%) 186\left(\mathrm{M}^{+}, 63 \%\right) 185$ (58), 171 (21), 155 (66), 32 (100); HRMS: calcd for $\mathrm{C}_{13} \mathrm{H}_{14} \mathrm{O}$ [M+1: 186.1045; found: 186.1040. 


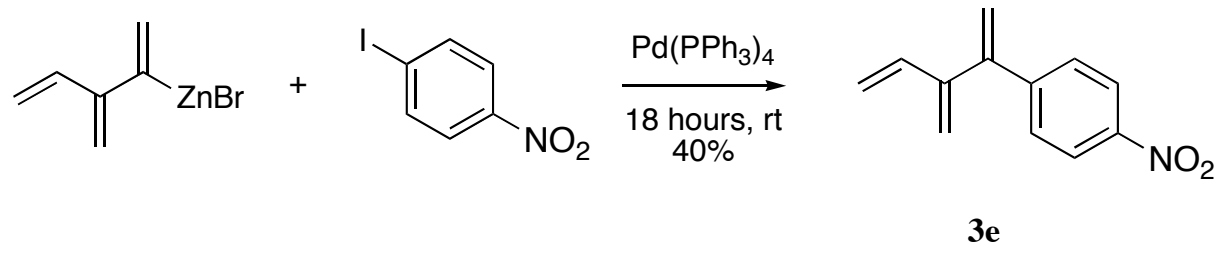

2-[3]Dendralenylmagnesium chloride 11 (20 mL of a $0.5 \mathrm{M}$ THF solution, $10 \mathrm{mmol}, 2$ equiv) was added dropwise to a solution of anhydrous zinc bromide (2.25 g, $10 \mathrm{mmol}, 2$ equiv) in anhydrous THF $(5 \mathrm{~mL})$ at $0{ }^{\circ} \mathrm{C}$ under nitrogen. The reaction mixture was allowed to warm to room temperature for 10 minutes, and 4-iodonitrobenzene (1.25 g, $5.0 \mathrm{mmol}, 1$ equiv) was added, followed by tetrakis(triphenylphosphine)palladium(0) (120 mg, $0.10 \mathrm{mmol}, 0.02$ equiv), and stirring was continued for 18 hours. The reaction mixture was poured into water $(100 \mathrm{~mL})$ and extracted with $30-40^{\circ} \mathrm{C}$ petrol $(2 \times 50 \mathrm{~mL})$. The organic extracts were washed with sat. brine $(100 \mathrm{~mL})$, dried over anhydrous magnesium sulphate and concentrated under reduced pressure $\left(0{ }^{\circ} \mathrm{C}, 30 \mathrm{mmHg}\right)$. The resulting oil was subjected to flash chromatography (0 - 50\% $\mathrm{CH}_{2} \mathrm{Cl}_{2} /$ hexanes) to give 2-(4-nitrophenyl)[3]dendralene 3e as a colourless oil (401 mg, 40\%). ${ }^{1} \mathrm{H}$ NMR (300 MHz, $\left.\mathrm{CDCl}_{3}\right) \delta 8.10-8.17$ (2 H, m), 7.50-7.57 (2 H, m), 6.46 (1 H, dd, $J=17.5,10.6 \mathrm{~Hz}), 5.68$ (1 H, br. s), 5.44 (1 H, br. s), 5.37 (1 H, br. s), 5.21 (1 H, br. s), $5.08(1 \mathrm{H}, \mathrm{d}, J=10.5 \mathrm{~Hz})$, and $4.96(1 \mathrm{H}, \mathrm{d}, J=17.5 \mathrm{~Hz}) \mathrm{ppm} ;{ }^{13} \mathrm{C} \mathrm{NMR}\left(75 \mathrm{MHz}, \mathrm{CDCl}_{3}\right) \delta$ $147.4(\mathrm{C}), 146.5(\mathrm{C}), 146.5(\mathrm{C}), 137.1(\mathrm{C}), 137.0(\mathrm{CH}), 127.7(\mathrm{CH}), 123.8(\mathrm{CH}), 119.7\left(\mathrm{CH}_{2}\right), 118.7$ $\left(\mathrm{CH}_{2}\right)$, and $118.2\left(\mathrm{CH}_{2}\right)$ ppm; IR (thin film): $\nu_{\max } 2850,1595,1517$ and $1344 \mathrm{~cm}^{-1}$; UV/vis (hexane) $\lambda_{\max }$ $198 \mathrm{~nm}(\varepsilon=60,000), 218 \mathrm{~nm}(\varepsilon=50,000), 291 \mathrm{~nm}(\varepsilon=27,000)$. EIMS $(70 \mathrm{eV}) \mathrm{m} / z(\%) 201\left(\mathrm{M}^{+}\right.$, 17\%), 185 (28), 184 (89), 155 (92), 154 (100), 153 (94); HRMS: calcd for $\mathrm{C}_{12} \mathrm{H}_{11} \mathrm{NO}_{2}\left[\mathrm{M}^{+}\right]$: 201.0790; found: 201.0795 . 


\section{2-Ethoxy[3]dendralene 3f, Pathway (i)}
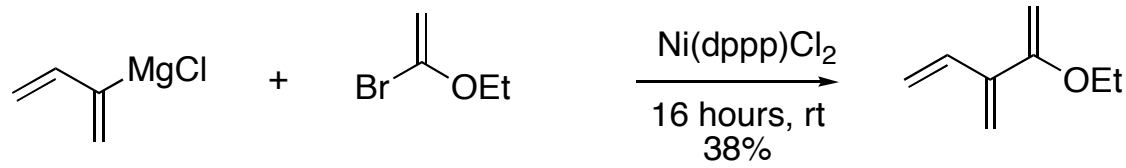

3f

1-Bromo-1-ethyoxyethylene was prepared by modifications to the reported procedure. ${ }^{8}$ A mixture of ethoxyacetylene $(0.80 \mathrm{~g}, 11.4 \mathrm{mmol}, 1.14$ equiv), anhydrous methanol (0.40 mL, $9.86 \mathrm{mmol}, 0.99$ equiv) and anhydrous $\mathrm{CH}_{2} \mathrm{Cl}_{2}(10 \mathrm{~mL})$ was cooled to $-78{ }^{\circ} \mathrm{C}$. Bromotrimethylsilane $(0.8 \mathrm{~mL}, 6.06$ mmol, 0.61 equiv) was added dropwise and the reaction mixture was allowed to warm to room temperature for one hour. The reaction mixture was concentrated under reduced pressure $\left(0{ }^{\circ} \mathrm{C}, 30\right.$ $\mathrm{mmHg})$. The resulting oil was mixed with 2-(1,3-butadienyl)magnesium chloride (10 mL of a $1 \mathrm{M}$ THF solution, $10 \mathrm{mmol}, 1$ equiv), $\mathrm{Ni}(\mathrm{dppp}) \mathrm{Cl}_{2}$ (40 $\mathrm{mg}, 0.074 \mathrm{mmol}, 0.01$ equiv) was added and the mixture was stirred for 18 hours under nitrogen. The reaction mixture was poured into $5 \% \mathrm{Na}_{2} \mathrm{CO}_{3}$ solution $(100 \mathrm{~mL})$ and $30-40{ }^{\circ} \mathrm{C}$ petrol $(100 \mathrm{~mL})$ and stirred for 15 minutes. The organic layer was dried over anhydrous $\mathrm{K}_{2} \mathrm{CO}_{3}$ and concentrated under reduced pressure $\left(0{ }^{\circ} \mathrm{C}, 30 \mathrm{mmHg}\right)$ to give an oil which was subjected to a bulb-to-trap distillation ( $\mathrm{rt}, 1 \mathrm{mmHg}$ ) to give 2-ethoxy[3]dendralene $\mathbf{3 f}$ as a colourless oil (283 mg, 38\%). The compound had identical ${ }^{1} \mathrm{H}$ and ${ }^{13} \mathrm{C}$ n.m.r. spectra to those reported. ${ }^{9}{ }^{1} \mathrm{H}$ NMR $\left(300 \mathrm{MHz}, \mathrm{CDCl}_{3}\right) \delta 6.41(1 \mathrm{H}, \mathrm{dd}, J=17.3,10.7 \mathrm{~Hz}), 5.46(1 \mathrm{H}, \mathrm{dd}, J=17.3,1.8 \mathrm{~Hz}), 5.44(1 \mathrm{H}, \mathrm{d}, J$ $=1.9 \mathrm{~Hz}), 5.24(1 \mathrm{H}, \mathrm{s}), 5.16(1 \mathrm{H}, \mathrm{dd}, J=10.7,1.5 \mathrm{~Hz}), 4.27(1 \mathrm{H}, \mathrm{d}, J=1.5 \mathrm{~Hz}), 4.16(1 \mathrm{H}, \mathrm{d}, J=$ $1.9 \mathrm{~Hz}), 3.80(2 \mathrm{H}, \mathrm{q}, J=6.9 \mathrm{~Hz})$ and $1.38(3 \mathrm{H}, \mathrm{q}, J=6.9 \mathrm{~Hz}) \mathrm{ppm} ;{ }^{13} \mathrm{C} \mathrm{NMR}\left(75 \mathrm{MHz}, \mathrm{CDCl}_{3}\right) \delta$ $159.3(\mathrm{C}), 143.2(\mathrm{C}), 135.5(\mathrm{C}), 116.6\left(\mathrm{CH}_{2}\right), 114.6\left(\mathrm{CH}_{2}\right), 84.8\left(\mathrm{CH}_{2}\right), 63.0\left(\mathrm{CH}_{2}\right)$ and $14.5\left(\mathrm{CH}_{3}\right) \mathrm{ppm}$. 


\section{2-Carbomethoxy[3]dendralene 3g, Pathway (iv)}
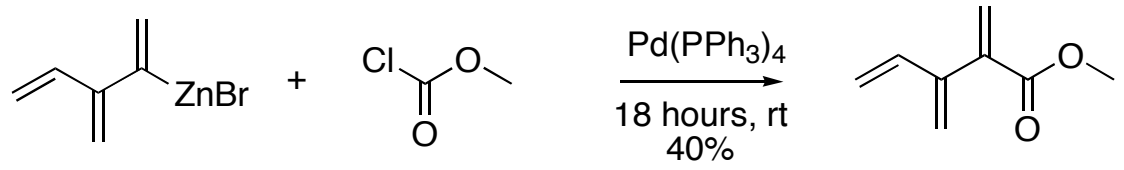

$3 g$

2-[3]Dendralenylmagnesium chloride 11 (10 mL of a $0.5 \mathrm{M}$ THF solution, $5 \mathrm{mmol}$ ) was added dropwise to a solution of anhydrous zinc bromide $(1.13 \mathrm{~g}, 5 \mathrm{mmol})$ in anhydrous $\mathrm{THF}(5 \mathrm{~mL})$ at $-78^{\circ} \mathrm{C}$ under nitrogen. The reaction mixture was allowed to warm to room temperature for 30 minutes then cooled to $-78{ }^{\circ} \mathrm{C}$. Methyl chloroformate $(0.40 \mathrm{~mL}, 490 \mathrm{mg}, 5.18 \mathrm{mmol})$ was added followed by tetrakis(triphenylphosphine)palladium(0) $(75 \mathrm{mg}, 0.065 \mathrm{mmol})$ and the reaction mixture was stirred for a further 30 minutes at $-78{ }^{\circ} \mathrm{C}$. The reaction was allowed to warm to room temperature for 18 hours. The reaction mixture was poured into water $(50 \mathrm{~mL})$ and diethyl ether $(50 \mathrm{~mL})$. A small amount of $1 \mathrm{M}$ $\mathrm{HCl}$ (aq.) was added to break up the emulsion. The organic layer was washed with water $(50 \mathrm{~mL})$, dried over anhydrous magnesium sulfate and concentrated under reduced pressure $\left(0{ }^{\circ} \mathrm{C}, 30 \mathrm{mmHg}\right)$ to give an oil which was subjected to radial chromatography. Elution with $2.5 \% \mathrm{EtOAc} / 40-60{ }^{\circ} \mathrm{C}$ petrol gave 2-carbomethoxy[3]dendralene $\mathbf{3 g}$ as a colourless oil $(278 \mathrm{mg}, 40 \%)$. $\mathrm{Rf} 0.14\left(25: 75 \mathrm{CHCl}_{3}: 40-\right.$ $60{ }^{\circ} \mathrm{C}$ petrol) ${ }^{1} \mathrm{H}$ NMR $\left(300 \mathrm{MHz}, \mathrm{CDCl}_{3}\right) \delta 6.47(1 \mathrm{H}, \mathrm{dd}, J=17.2,10.3 \mathrm{~Hz}), 6.32(1 \mathrm{H}, \mathrm{d}, J=1.7$ Hz), $5.70(1 \mathrm{H}, \mathrm{d}, J=1.7 \mathrm{~Hz}), 5.28$ (1 H, br. s), 5.17 (1 H, br. s), $5.14(1 \mathrm{H}, \mathrm{d}, \mathrm{J}=10.3 \mathrm{~Hz}) 5.05$ (1 H, d, $J=17.2 \mathrm{~Hz}), 3.75(3 \mathrm{H}, \mathrm{s}) \mathrm{ppm} ;{ }^{13} \mathrm{C} \mathrm{NMR}\left(75 \mathrm{MHz}, \mathrm{CDCl}_{3}\right) \delta 166.9(\mathrm{C}), 144.4(\mathrm{C}), 139.8(\mathrm{C}), 137.2$ (CH), $128.3\left(\mathrm{CH}_{2}\right), 118.9\left(\mathrm{CH}_{2}\right), 116.4\left(\mathrm{CH}_{2}\right), 52.2\left(\mathrm{CH}_{3}\right)$ ppm; IR (thin film): $v_{\max } 2953,1727 \mathrm{~cm}^{-1}$; $\mathrm{UV} / \mathrm{vis}$ (hexane) $\lambda_{\max } 219 \mathrm{~nm}(\varepsilon=27,000)$. EIMS (70 eV) $m / z(\%) 138\left(\mathrm{M}^{+}, 69 \%\right), 107(25), 32(100)$; HRMS: calcd for $\mathrm{C}_{8} \mathrm{H}_{10} \mathrm{O}_{2}\left[\mathrm{M}^{+}\right]$: 138.0681; found: 138.0687 . 


\section{Diels-Alder Adducts of $N$-methylmaleimide}

\section{General Procedure for the preparation of the Diels-Alder Adducts with $N$-methylmaleimide}

$N$-methylmaleimide ( 1 or 5 equiv) was added to a stirred solution of 2-substituted [3]dendralene 3 a-e, g-h (1 equiv) in $\mathrm{CH}_{2} \mathrm{Cl}_{2}$ or $\mathrm{CDCl}_{3}(0.01 \mathrm{M}-1 \mathrm{M})$ at $25^{\circ} \mathrm{C}$. When the reaction was deemed complete by ${ }^{1} \mathrm{H}$ NMR analysis (16 - 96 hours) the solvent was removed under reduced pressure and the residue was purified by flash chromatography. Regioisomers resulting from a single cycloaddition and diastereomers resulting from a double cycloaddition were further subjected to HPLC for purification where necessary.

\section{Diels-Alder reaction of 2-chloro[3]dendralene 3a with $N$-methylmaleimide}

Use of one molar equivalent of $N$-methylmaleimide gave a $51 \%$ isolated yield of mono-adduct $13 \mathbf{a}$ and mono-adduct 16a in a 77:23 ratio.

Use of five molar equivalents of $N$-methylmaleimide gave a $65 \%$ isolated yield of bis-adduct 14a, bisadduct 15a and mono-adduct 16a in a 61:12:27 ratio.

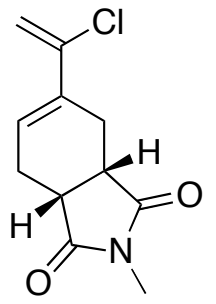

13a

Mono-adduct 13a was isolated as colourless oil; $\mathrm{Rf} 0.36\left(95: 5 \mathrm{CH}_{2} \mathrm{Cl}_{2}: \mathrm{Et}_{2} \mathrm{O}\right) ;{ }^{1} \mathrm{H}$ NMR (300 MHz, $\left.\mathrm{CDCl}_{3}\right) \delta 6.43(1 \mathrm{H}, \mathrm{m}), 5.50(1 \mathrm{H}, \mathrm{d}, J=1.8 \mathrm{~Hz}), 5.38(1 \mathrm{H}, \mathrm{d}, J$ $=1.8 \mathrm{~Hz}), 3.15(2 \mathrm{H}, \mathrm{m}), 2.94(3 \mathrm{H}, \mathrm{s}), 2.86(2 \mathrm{H}, \mathrm{m})$ and $2.35(2 \mathrm{H}, \mathrm{m}) \mathrm{ppm} ;{ }^{13} \mathrm{C}$ NMR (125 MHz, $\left.\mathrm{CDCl}_{3}\right) \delta 179.3(\mathrm{C}), 179.1(\mathrm{C}), 138.9(\mathrm{C}), 135.0(\mathrm{C}), 127.9(\mathrm{CH})$, 112.4 $\left(\mathrm{CH}_{2}\right), 39.6(\mathrm{CH}), 38.7(\mathrm{CH}), 25.3\left(\mathrm{CH}_{2}\right), 24.8\left(\mathrm{CH}_{3}\right)$ and $24.7\left(\mathrm{CH}_{2}\right)$ ppm; IR (thin film): $v_{\max } 2950,1698$ and $1435 \mathrm{~cm}^{-1}$; EIMS $(70 \mathrm{eV}) \mathrm{m} / z(\%) 227\left(\mathrm{M}^{+},{ }^{37} \mathrm{Cl}\right.$, 12\%), $225\left(\mathrm{M}^{+},{ }^{35} \mathrm{Cl}, 36\right), 190$ (15) and 105 (100); HRMS: calcd for $\mathrm{C}_{11} \mathrm{H}_{12}{ }^{35} \mathrm{ClNO}_{2}\left[\mathrm{M}^{+}\right]$: 225.0557; found: 225.0554 . 


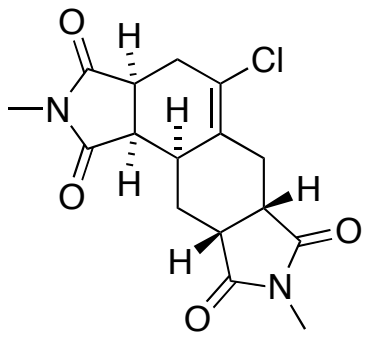

$14 a$

x-ray

Bis-adduct 14a was isolated as colourless prisms, mp $176-177{ }^{\circ} \mathrm{C}(1,2-$ dichloroethane:hexane); Rf 0.07 (95:5 $\left.\mathrm{CH}_{2} \mathrm{Cl}_{2}: \mathrm{Et}_{2} \mathrm{O}\right) ;{ }^{1} \mathrm{H}$ NMR $(300 \mathrm{MHz}$, $\left.\mathrm{CDCl}_{3}\right) \delta 3.24(2 \mathrm{H}, \mathrm{m}), 3.13(2 \mathrm{H}, \mathrm{m}), 2.96(2 \mathrm{H}, \mathrm{m}), 2.95(3 \mathrm{H}, \mathrm{s}), 2.92(3 \mathrm{H}$, s), $2.71(1 \mathrm{H}, \mathrm{m}), 2.47(2 \mathrm{H}, \mathrm{m})$ and $2.16(2 \mathrm{H}, \mathrm{m}) \mathrm{ppm} ;{ }^{13} \mathrm{C} \mathrm{NMR}(75 \mathrm{MHz}$, $\left.\mathrm{CDCl}_{3}\right) \delta 179.7(\mathrm{C}), 179.1(\mathrm{C}), 178.1(\mathrm{C}), 177.1(\mathrm{C}), 130.5(\mathrm{C}), 125.4(\mathrm{C}), 42.9$ $(\mathrm{CH}), 40.3(\mathrm{CH}), 38.9(\mathrm{CH}), 38.7(\mathrm{CH}), 34.9(\mathrm{CH}), 33.4\left(\mathrm{CH}_{2}\right), 25.3\left(\mathrm{CH}_{2}\right)$, $25.2\left(\mathrm{CH}_{3}\right), 25.2\left(\mathrm{CH}_{3}\right)$ and $23.8\left(\mathrm{CH}_{2}\right)$ ppm; IR (thin film): $v_{\max } 2945,2846$ and $1695 \mathrm{~cm}^{-1}$; EIMS $(70 \mathrm{eV}) \mathrm{m} / z(\%) 338\left(\mathrm{M}^{+},{ }^{37} \mathrm{Cl}, 20 \%\right), 336\left(\mathrm{M}^{+},{ }^{35} \mathrm{Cl}, 61\right), 301$ (26) and $49(100)$; HRMS: calcd for $\mathrm{C}_{16} \mathrm{H}_{17}{ }^{35} \mathrm{ClN}_{2} \mathrm{O}_{4}\left[\mathrm{M}^{+}\right]$: 336.0877; found: 336.0878 .

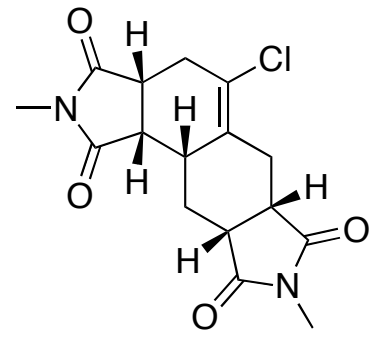

$15 \mathbf{a}$

Bis-adduct 15a was isolated as a colourless oil; Rf 0.07 (95:5 $\left.\mathrm{CH}_{2} \mathrm{Cl}_{2}: \mathrm{Et}_{2} \mathrm{O}\right) ;{ }^{1} \mathrm{H} \mathrm{NMR}\left(500 \mathrm{MHz}, \mathrm{CDCl}_{3}\right) \delta 3.28(1 \mathrm{H}, \mathrm{dd}, J=15.0,6.5$ Hz), $3.21(1 \mathrm{H}, \mathrm{m}), 3.12(2 \mathrm{H}, \mathrm{m}), 3.01(3 \mathrm{H}, \mathrm{s}), 2.98(1 \mathrm{H}, \mathrm{d}, J=1.5 \mathrm{~Hz})$, $2.93(3 \mathrm{H}, \mathrm{s}), 2.86(2 \mathrm{H}, \mathrm{m}), 2.67(1 \mathrm{H}, \mathrm{m}), 2.40(2 \mathrm{H}, \mathrm{m})$ and $1.94(1 \mathrm{H}, \mathrm{br}$. $\mathrm{t}, J=11.5 \mathrm{~Hz}) \mathrm{ppm} ;{ }^{13} \mathrm{C} \mathrm{NMR}\left(125 \mathrm{MHz}, \mathrm{CDCl}_{3}\right) \delta 178.7(\mathrm{C}), 178.3(\mathrm{C})$, $177.0(\mathrm{C}), 176.0(\mathrm{C}), 132.0(\mathrm{CH}), 124.6(\mathrm{C}), 43.1(\mathrm{CH}), 40.7(\mathrm{CH}), 40.0$ $(\mathrm{CH}), 38.4(\mathrm{CH}), 37.9(\mathrm{CH}), 33.5\left(\mathrm{CH}_{2}\right), 25.3\left(\mathrm{CH}_{2}\right) 25.2\left(\mathrm{CH}_{3}\right), 25.0\left(\mathrm{CH}_{3}\right)$ and $24.8\left(\mathrm{CH}_{2}\right) \mathrm{ppm}$; IR (thin film): $v_{\max } 2926,1775,1697$ and $1436 \mathrm{~cm}^{-1}$; EIMS $(70 \mathrm{eV}) \mathrm{m} / \mathrm{z}(\%) 338$ $\left(\mathrm{M}^{+},{ }^{37} \mathrm{Cl}, 6 \%\right), 336\left(\mathrm{M}^{+},{ }^{35} \mathrm{Cl}, 18\right)$ and 69 (100); HRMS: calcd for $\mathrm{C}_{16} \mathrm{H}_{17}{ }^{35} \mathrm{ClN}_{2} \mathrm{O}_{4}\left[\mathrm{M}^{+}\right]$: 336.0877; found: 336.0880 .

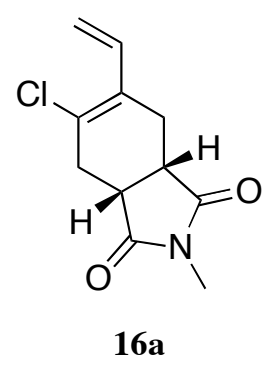

Mono-adduct 16a was isolated as a colourless oil; $\mathrm{Rf} 0.36\left(95: 5 \mathrm{CH}_{2} \mathrm{Cl}_{2}: \mathrm{Et}_{2} \mathrm{O}\right)$; ${ }^{1} \mathrm{H}$ NMR (300 MHz, $\left.\mathrm{CDCl}_{3}\right) \delta 6.81(1 \mathrm{H}, \mathrm{dd}, J=17.4,11.1 \mathrm{~Hz}), 5.39(1 \mathrm{H}, \mathrm{br}$. d, $J=17.4 \mathrm{~Hz}), 5.23(1 \mathrm{H}$, br. d, $J=11.1 \mathrm{~Hz}), 3.18(2 \mathrm{H}, \mathrm{m}), 2.98(2 \mathrm{H}, \mathrm{m})$, $2.97(3 \mathrm{H}, \mathrm{s}), 2.81(1 \mathrm{H}, \mathrm{m})$ and $2.35(1 \mathrm{H}, \mathrm{m}) \mathrm{ppm} ;{ }^{13} \mathrm{C} \mathrm{NMR}(125 \mathrm{MHz}$, $\left.\mathrm{CDCl}_{3}\right) \delta 178.8(\mathrm{C}), 178.7(\mathrm{C}), 131.9(\mathrm{C}), 130.6(\mathrm{CH}), 127.7(\mathrm{C}), 115.9\left(\mathrm{CH}_{2}\right)$, $39.9(\mathrm{CH}), 39.1(\mathrm{CH}), 33.5\left(\mathrm{CH}_{2}\right), 25.3\left(\mathrm{CH}_{3}\right)$ and $24.6\left(\mathrm{CH}_{2}\right)$ ppm; IR (thin film): $v_{\max } 2924,1698,1630$ and $1435 \mathrm{~cm}^{-1}$; EIMS $(70 \mathrm{eV}) \mathrm{m} / z(\%) 227\left(\mathrm{M}^{+},{ }^{37} \mathrm{Cl}, 14 \%\right), 225\left(\mathrm{M}^{+},{ }^{35} \mathrm{Cl}\right.$, 43), 190 (9), 140 (25) and 105 (100); HRMS: calcd for $\mathrm{C}_{11} \mathrm{H}_{12}{ }^{35} \mathrm{ClNO}_{2}\left[\mathrm{M}^{+}\right]$: 225.0557; found: 225.0557. 


\section{Diels-Alder reaction of 2-phenyl[3]dendralene $3 \mathrm{~b}$ with $N$-methylmaleimide}

Use of one molar equivalent of $N$-methylmaleimide gave a $90 \%$ isolated yield of mono-adduct $\mathbf{1 3 b}$ and mono-adduct $\mathbf{1 6 b}$ in a 71:29 ratio.

Use of five molar equivalents of $N$-methylmaleimide gave a $90 \%$ isolated yield of bis-adduct $\mathbf{1 4 b}$, bisadduct $\mathbf{1 5 b}$ and mono-adduct $\mathbf{1 6} \mathbf{b}$ in a 58:6:36 ratio.

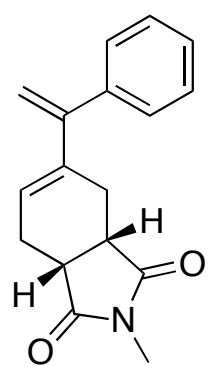

$13 \mathbf{b}$

Mono-adduct 13b was isolated as a colourless oil; Rf 0.30 (30:70 EtOAc:40 $60{ }^{\circ} \mathrm{C}$ petrol); ${ }^{1} \mathrm{H}$ NMR $\left(300 \mathrm{MHz}, \mathrm{CDCl}_{3}\right) \delta 7.28(3 \mathrm{H}, \mathrm{m}), 7.13(2 \mathrm{H}, \mathrm{m}), 5.72$ (1 H, m), $5.28(1 \mathrm{H}, \mathrm{s}), 5.11(1 \mathrm{H}, \mathrm{s}), 3.21(1 \mathrm{H}, \mathrm{m}), 3.11(1 \mathrm{H}, \mathrm{m}), 2.99(3 \mathrm{H}, \mathrm{s})$, $2.92(1 \mathrm{H}, \mathrm{dd}, J=15.0,3.3 \mathrm{~Hz}), 2.65(1 \mathrm{H}, \mathrm{ddd}, J=15.0,6.0,3.0 \mathrm{~Hz})$ and 2.39 $(2 \mathrm{H}, \mathrm{m}) \mathrm{ppm} ;{ }^{13} \mathrm{C} \mathrm{NMR}\left(125 \mathrm{MHz}, \mathrm{CDCl}_{3}\right) \delta 180.1$ (C) 179.7 (C) 149.3 (C), $140.8(\mathrm{C}), 139.6(\mathrm{C}), 128.5(2 \times \mathrm{CH}), 128.1(2 \times \mathrm{CH}), 127.6(\mathrm{CH}), 126.5(\mathrm{CH})$, $113.4\left(\mathrm{CH}_{2}\right), 39.9(\mathrm{CH}), 39.1(\mathrm{CH}), 25.7\left(\mathrm{CH}_{2}\right), 25.1\left(\mathrm{CH}_{3}\right)$ and $24.8\left(\mathrm{CH}_{2}\right)$ ppm; IR (thin film): $v_{\max }$ 2923, 1775 and $1695 \mathrm{~cm}^{-1}$; EIMS (70 eV) m/z (\%) $267\left(\mathrm{M}^{+}, 100 \%\right), 181(25)$, 155 (76), 112 (27) and 77 (25); HRMS: calcd for $\mathrm{C}_{17} \mathrm{H}_{17} \mathrm{NO}_{2}\left[\mathrm{M}^{+}\right]$: 267.1259; found: 267.1262.

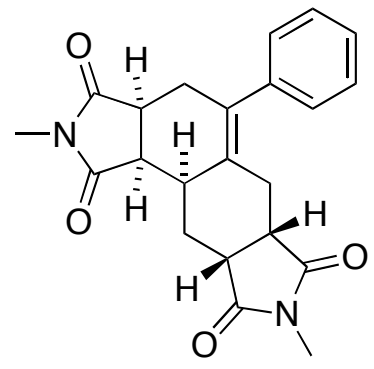

$14 b$

Bis-adduct 14b was isolated as a colourless oil; Rf 0.06 (30:70 EtOAc:40 $-60{ }^{\circ} \mathrm{C}$ petrol); ${ }^{1} \mathrm{H}$ NMR (500 MHz, $\left.\mathrm{CDCl}_{3}\right) \delta 7.25(2 \mathrm{H}, \mathrm{m}), 7.16(1 \mathrm{H}$, m), $6.97(1 \mathrm{H}, \mathrm{m}), 3.18(1 \mathrm{H}, \mathrm{m}), 3.11(1 \mathrm{H}, \mathrm{dd}, J=7.5,7.5 \mathrm{~Hz}), 3.01(3 \mathrm{H}$, m), $2.93(3 \mathrm{H}, \mathrm{s}), 2.87(3 \mathrm{H}, \mathrm{s}), 2.84(1 \mathrm{H}, \mathrm{m}), 2.78(1 \mathrm{H}, \mathrm{m}), 2.37(2 \mathrm{H}, \mathrm{m})$ and $2.11(2 \mathrm{H}, \mathrm{m}) \mathrm{ppm} ;{ }^{13} \mathrm{C} \mathrm{NMR}\left(125 \mathrm{MHz}, \mathrm{CDCl}_{3}\right) \delta 180.1$ (C) 179.6 (C) $179.4(\mathrm{C}), 177.9$ (C), 139.4 (C), 135.8 (C), 130.5 (C), $128.5(2 \times \mathrm{CH})$, $127.8(2 \times \mathrm{CH}), 127.1(\mathrm{CH}), 43.5(\mathrm{CH}), 40.4(\mathrm{CH}), 39.3(\mathrm{CH}), 39.0(\mathrm{CH})$,

$34.4(\mathrm{CH}), 32.0\left(\mathrm{CH}_{2}\right), 25.0\left(\mathrm{CH}_{3}\right), 24.9\left(\mathrm{CH}_{3}\right), 24.8\left(\mathrm{CH}_{2}\right)$ and $23.4\left(\mathrm{CH}_{2}\right)$ ppm; IR (thin film): $v_{\max }$ 2947, 1773, 1694 and $1599 \mathrm{~cm}^{-1}$; EIMS (70 eV) m/z (\%) $378\left(\mathrm{M}^{+}, 100 \%\right), 292$ (69), 267 (58), 165 (50) and 112 (79); HRMS: calcd for $\mathrm{C}_{22} \mathrm{H}_{22} \mathrm{~N}_{2} \mathrm{O}_{4}\left[\mathrm{M}^{+}\right]$: 378.1580; found: 378.1579 . 


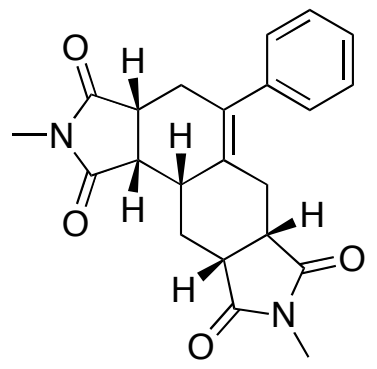

15b

Bis-adduct 15b was isolated as a colourless oil; Rf 0.06 (30:70 EtOAc:40 - $60{ }^{\circ} \mathrm{C}$ petrol); ${ }^{1} \mathrm{H}$ NMR (500 MHz, $\left.\mathrm{CDCl}_{3}\right) \delta 7.29(2 \mathrm{H}, \mathrm{m}), 7.22(1 \mathrm{H}$, m), $6.96(1 \mathrm{H}, \mathrm{m}), 3.22$ (1 H, br. dd, $J=7.0,7.0 \mathrm{~Hz}), 3.13(1 \mathrm{H}$, br. dd, $J=$ 8.5, 5.0 Hz), $3.00(3 \mathrm{H}, \mathrm{m}), 2.95(3 \mathrm{H}, \mathrm{s}), 2.88$ (3 H, s), $2.80(2 \mathrm{H}, \mathrm{m}), 2.55$ $(1 \mathrm{H}, \mathrm{m}), 2.44(3 \mathrm{H}, \mathrm{m})$ and $1.94(1 \mathrm{H}, \mathrm{br} . \mathrm{dd}, J=11.0,11.0 \mathrm{~Hz}) \mathrm{ppm} ;{ }^{13} \mathrm{C}$ NMR (125 MHz, $\mathrm{CDCl}_{3}$ ) $\delta 179.3$ (C) 178.9 (C) 178.9 (C), 177.7 (C), $139.7(\mathrm{C}), 134.8(\mathrm{C}), 132.4(\mathrm{C}), 128.7(2 \times \mathrm{CH}), 127.9(2 \times \mathrm{CH}), 127.3$ $(\mathrm{CH}), 43.5(\mathrm{CH}), 40.7(\mathrm{CH}), 40.1(\mathrm{CH}), 39.5(\mathrm{CH}), 37.3(\mathrm{CH}), 31.7\left(\mathrm{CH}_{2}\right), 25.2\left(\mathrm{CH}_{2}\right), 25.0\left(\mathrm{CH}_{2}\right)$, $24.9\left(\mathrm{CH}_{3}\right)$ and $24.9\left(\mathrm{CH}_{3}\right)$ ppm; IR (thin film): $v_{\max } 2951,1774$ and $1696 \mathrm{~cm}^{-1}$; EIMS $(70 \mathrm{eV}) \mathrm{m} / z(\%)$ $378\left(\mathrm{M}^{+}, 100 \%\right), 293$ (21), 267 (11), 165 (18) and 112 (26); HRMS: calcd for $\mathrm{C}_{22} \mathrm{H}_{22} \mathrm{~N}_{2} \mathrm{O}_{4}\left[\mathrm{M}^{+}\right]$: 378.1580; found: 378.1580 .

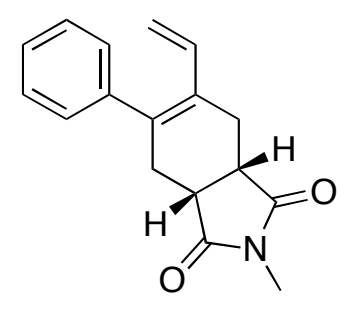

16b

Mono-adduct 16b was isolated as a colourless oil; Rf 0.30 (30:70 EtOAc:40 $60{ }^{\circ} \mathrm{C}$ petrol); ${ }^{1} \mathrm{H}$ NMR (500 MHz, $\left.\mathrm{CDCl}_{3}\right) \delta 7.25(2 \mathrm{H}, \mathrm{m}), 7.19(1 \mathrm{H}, \mathrm{m}), 7.04$ $(2 \mathrm{H}, \mathrm{m}), 6.45(1 \mathrm{H}, \mathrm{dd}, J=17.5,10.5 \mathrm{~Hz}), 5.29(1 \mathrm{H}, \mathrm{d}, J=17.5 \mathrm{~Hz}), 4.95$ (1 $\mathrm{H}, \mathrm{d}, J=10.5 \mathrm{~Hz}), 3.17(2 \mathrm{H}, \mathrm{m}), 3.07(1 \mathrm{H}, \mathrm{dd}, J=14.5,2.5 \mathrm{~Hz}), 2.93(1 \mathrm{H}$, $\mathrm{dd}, J=15.5,2.5 \mathrm{~Hz}), 2.89(3 \mathrm{H}, \mathrm{s}), 2.62(1 \mathrm{H}, \mathrm{dd}, J=15.0,6.0 \mathrm{~Hz})$ and $2.27(1$ $\mathrm{H}$, ddd, $J=13.0,6.5,1.5 \mathrm{~Hz}) \mathrm{ppm} ;{ }^{13} \mathrm{C} \mathrm{NMR}\left(125 \mathrm{MHz}, \mathrm{CDCl}_{3}\right) \delta 180.0(\mathrm{C})$, $179.6(\mathrm{C}), 140.3(\mathrm{C}), 137.5(\mathrm{C}), 134.3(\mathrm{CH}), 131.8(\mathrm{C}), 128.9(2 \times \mathrm{CH}), 128.2(2 \times \mathrm{CH}), 127.3(\mathrm{CH})$, $113.8\left(\mathrm{CH}_{2}\right) 40.0(\mathrm{CH}), 39.9(\mathrm{CH}), 32.0\left(\mathrm{CH}_{2}\right) 25.1\left(\mathrm{CH}_{3}\right)$ and $24.3\left(\mathrm{CH}_{2}\right)$ ppm; IR (thin film): $v_{\max }$ 2926, 1776, 1698 and $1436 \mathrm{~cm}^{-1}$; EIMS (70 eV) m/z (\%) $267\left(\mathrm{M}^{+}, 100 \%\right), 182$ (38) and $128(34)$; HRMS: calcd for $\mathrm{C}_{17} \mathrm{H}_{17} \mathrm{NO}_{2}\left[\mathrm{M}^{+}\right]$: 267.1259; found: 267.1257 .

\section{Diels-Alder reaction of 2-methyl[3]dendralene 3c with $N$-methylmaleimide}

Use of five molar equivalents of $N$-methylmaleimide gave a $64 \%$ isolated yield of bis-adduct $14 \mathbf{c}$, bisadduct $\mathbf{1 5 c}$ and mono-adduct $\mathbf{1 6 c}$ in a 56:8:36 ratio. 


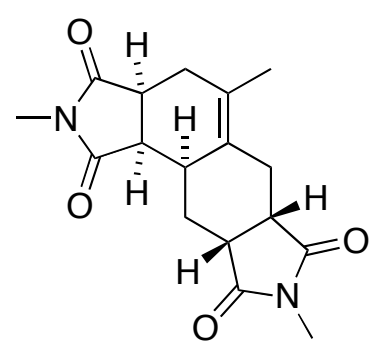

14c

Bis-adduct 14c was isolated as a colourless oil; Rf 0.06 (30:70 EtOAc:40 $60^{\circ} \mathrm{C}$ petrol); ${ }^{1} \mathrm{H}$ NMR (500 MHz, $\left.\mathrm{CDCl}_{3}\right) \delta 3.18(1 \mathrm{H}, \mathrm{m}), 3.08(1 \mathrm{H}, \mathrm{m})$, $3.01(1 \mathrm{H}, \mathrm{m}), 2.94(2 \mathrm{H}, \mathrm{m}), 2.90(3 \mathrm{H}, \mathrm{s}), 2.86(3 \mathrm{H}, \mathrm{s}), 2.65(1 \mathrm{H}, \mathrm{td}, J=$ 14.0, $5.5 \mathrm{~Hz}), 2.51(1 \mathrm{H}, \mathrm{dd}, J=14.5,1.5 \mathrm{~Hz}), 2.32(1 \mathrm{H}, \mathrm{ddd}, J=14.0,5.0$, $2.5 \mathrm{~Hz}), 2.10(2 \mathrm{H}, \mathrm{m}), 1.99(1 \mathrm{H}, \mathrm{m})$ and $1.65(3 \mathrm{H}, \mathrm{s}) \mathrm{ppm} ;{ }^{13} \mathrm{C}$ NMR $(125$ $\left.\mathrm{MHz}, \mathrm{CDCl}_{3}\right) \delta 180.2(\mathrm{C}), 179.9(\mathrm{C}), 179.4(\mathrm{C}), 178.0(\mathrm{C}), 130.6(\mathrm{C}), 127.1$ (C), $43.4(\mathrm{CH}), 39.9(\mathrm{CH}), 39.6(\mathrm{CH}), 39.1(\mathrm{CH}), 33.8(\mathrm{CH}), 31.0\left(\mathrm{CH}_{2}\right)$, $25.0\left(\mathrm{CH}_{3}\right), 24.9\left(\mathrm{CH}_{3}\right), 24.2\left(\mathrm{CH}_{2}\right), 23.7\left(\mathrm{CH}_{2}\right)$ and $19.0\left(\mathrm{CH}_{3}\right)$ ppm; IR (thin film): $v_{\max } 2945,2849$, 1773, 1694 and $1435 \mathrm{~cm}^{-1}$; EIMS (70 eV) $\mathrm{m} / z$ (\%) $316\left(\mathrm{M}^{+}, 100 \%\right), 301$ (50) and 230 (38); HRMS: calcd for $\mathrm{C}_{17} \mathrm{H}_{20} \mathrm{~N}_{2} \mathrm{O}_{4}\left[\mathrm{M}^{+}\right]$: 316.1423; found: 316.1423 .

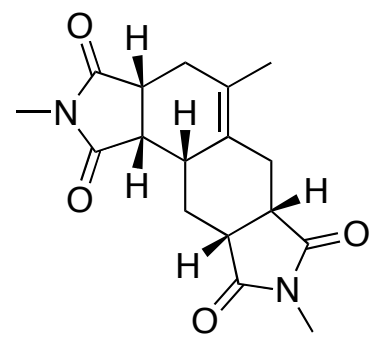

$15 \mathrm{c}$

Bis-adduct 15c was isolated as a colourless oil; Rf 0.06 (30:70 EtOAc:40 $60{ }^{\circ} \mathrm{C}$ petrol); ${ }^{1} \mathrm{H}$ NMR $\left(500 \mathrm{MHz}, \mathrm{CDCl}_{3}\right) \delta 3.09(2 \mathrm{H}, \mathrm{m}), 3.00(3 \mathrm{H}, \mathrm{s})$, $2.97(1 \mathrm{H}, \mathrm{m}), 2.88(3 \mathrm{H}, \mathrm{s}), 2.79(2 \mathrm{H}, \mathrm{m}), 2.62(1 \mathrm{H}, \mathrm{dd}, J=15.0,1.5 \mathrm{~Hz})$, $2.33(3 \mathrm{H}, \mathrm{m}), 2.23(1 \mathrm{H}, \mathrm{m}), 1.86(1 \mathrm{H}, \mathrm{dd}, J=11.0,11.0 \mathrm{~Hz})$ and $1.74(3$ $\mathrm{H}, \mathrm{s}) \mathrm{ppm} ;{ }^{13} \mathrm{C}$ NMR (125 MHz, $\left.\mathrm{CDCl}_{3}\right) \delta 179.5(\mathrm{C}), 179.4(\mathrm{C}), 179.1$ (C), $177.8(\mathrm{C}), 129.4(\mathrm{C}), 128.7(\mathrm{C}), 43.7(\mathrm{CH}), 40.4(\mathrm{CH}), 40.1(\mathrm{CH}), 39.5$ $(\mathrm{CH}), 36.6(\mathrm{CH}), 31.1\left(\mathrm{CH}_{2}\right), 25.2\left(\mathrm{CH}_{2}\right), 25.0\left(\mathrm{CH}_{3}\right), 24.9\left(\mathrm{CH}_{3}\right), 24.0$ $\left(\mathrm{CH}_{2}\right)$ and $19.3\left(\mathrm{CH}_{3}\right) \mathrm{ppm}$; IR (thin film): $v_{\max } 2925,1773,1694$ and $1435 \mathrm{~cm}^{-1}$; EIMS (70 eV) $\mathrm{m} / z(\%)$ $316\left(\mathrm{M}^{+}, 100 \%\right), 231$ (24) and 205 (34); HRMS: calcd for $\mathrm{C}_{17} \mathrm{H}_{20} \mathrm{~N}_{2} \mathrm{O}_{4}\left[\mathrm{M}^{+}\right]$: 316.1423; found: 316.1429 .

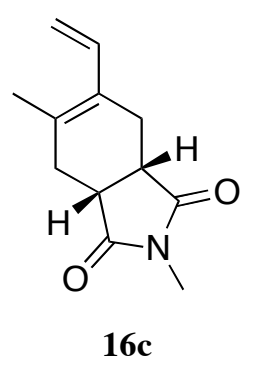

Mono-adduct 16c was isolated as a colourless oil; Rf 0.29 (30:70 EtOAc:40 - 60 ${ }^{\circ} \mathrm{C}$ petrol); ${ }^{1} \mathrm{H}$ NMR $\left(500 \mathrm{MHz}, \mathrm{CDCl}_{3}\right) \delta 6.64(1 \mathrm{H}, \mathrm{dd}, J=17.5,11.0 \mathrm{~Hz}), 5.21(1$ $\mathrm{H}, \mathrm{d}, J=17.0 \mathrm{~Hz}), 5.00(1 \mathrm{H}, \mathrm{d}, J=11.0 \mathrm{~Hz}), 3.07(2 \mathrm{H}, \mathrm{m}), 2.89(3 \mathrm{H}, \mathrm{s}), 2.87(1$ $\mathrm{H}, \mathrm{dd}, J=15.0,3.0 \mathrm{~Hz}), 2.51(1 \mathrm{H}, \mathrm{dd}, J=15.5,3.5 \mathrm{~Hz}), 2.35(1 \mathrm{H}, \mathrm{dd}, J=15.5$, $7.0 \mathrm{~Hz}), 2.17(1 \mathrm{H}, \mathrm{m})$ and $1.80(3 \mathrm{H}, \mathrm{s}) \mathrm{ppm} ;{ }^{13} \mathrm{C} \mathrm{NMR}\left(125 \mathrm{MHz}, \mathrm{CDCl}_{3}\right) \delta 179.9$ (C), $179.8(\mathrm{C}), 133.0(\mathrm{C}), 132.7(\mathrm{CH}), 129.1(\mathrm{C}), 112.3\left(\mathrm{CH}_{2}\right), 39.7(\mathrm{CH}), 39.4$ 
$(\mathrm{CH}), 31.5\left(\mathrm{CH}_{2}\right), 25.0\left(\mathrm{CH}_{3}\right), 23.4\left(\mathrm{CH}_{2}\right)$ and $19.2\left(\mathrm{CH}_{3}\right) \mathrm{ppm}$; IR (thin film): $v_{\max } 2946,2854,1776$, 1698 and $1434 \mathrm{~cm}^{-1}$; EIMS (70 eV) m/z (\%) $205\left(\mathrm{M}^{+}, 100 \%\right), 190$ (6), 178 (4) and 120 (67); HRMS: calcd for $\mathrm{C}_{12} \mathrm{H}_{15} \mathrm{NO}_{2}\left[\mathrm{M}^{+}\right]$: 205.1103; found: 205.1104 .

\section{Diels-Alder reaction with $p$-anisyl[3]dendralene $3 d$ with $N$-methylmaleimide}

Use of one molar equivalent of $N$-methylmaleimide gave a $86 \%$ (80\% conversion) isolated yield of mono-adduct 13d and mono-adduct $\mathbf{1 6 d}$ in a 73:27 ratio.

Use of five molar equivalents of $N$-methylmaleimide gave a $67 \%$ isolated yield of bis-adduct $\mathbf{1 4 d}$, bisadduct $\mathbf{1 5 d}$ and mono-adduct $\mathbf{1 6 d}$ in a 65:7:28 ratio.

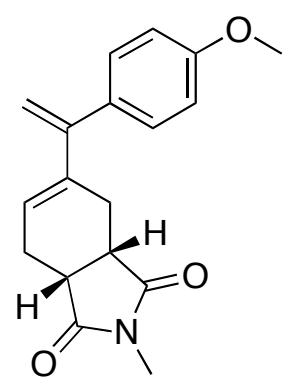

$13 d$

Mono-adduct 13d was isolated as a colourless oil; $\mathrm{Rf} 0.43$ (95:5 $\left.\mathrm{CH}_{2} \mathrm{Cl}_{2}: \mathrm{Et}_{2} \mathrm{O}\right) ;{ }^{1} \mathrm{H} \mathrm{NMR}\left(500 \mathrm{MHz}, \mathrm{CDCl}_{3}\right) \delta 7.07(2 \mathrm{H}, \mathrm{d}, J=9.0 \mathrm{~Hz}), 6.45$ $(2 \mathrm{H}, \mathrm{d}, J=9.0 \mathrm{~Hz}), 5.77(1 \mathrm{H}, \mathrm{m}), 5.20(1 \mathrm{H}, \mathrm{s}), 5.06(1 \mathrm{H}, \mathrm{s}) 3.81(3 \mathrm{H}, \mathrm{s})$, $3.19(1 \mathrm{H}, \mathrm{m}), 3.10(1 \mathrm{H}, \mathrm{m}), 2.89$ (1 H, dd, $J=15.5,3.5 \mathrm{~Hz}), 2.65(1 \mathrm{H}$, ddd, $J=16.0,7.0,3.5 \mathrm{~Hz}), 2.42(1 \mathrm{H}, \mathrm{qt}, J=7.0,2.5 \mathrm{~Hz})$ and $2.34(1 \mathrm{H}, \mathrm{m})$ ppm; ${ }^{13} \mathrm{C}$ NMR (125 MHz, $\left.\mathrm{CDCl}_{3}\right) \delta 180.2$ (C) 179.7 (C) 159.2 (C), 148.8 (C), $139.9(\mathrm{CH}), 133.1(\mathrm{C}), 129.6(2 \times \mathrm{CH}), 126.2(\mathrm{CH}), 113.5(2 \times \mathrm{CH})$, $112.5\left(\mathrm{CH}_{2}\right), 55.4\left(\mathrm{CH}_{3}\right), 39.9(\mathrm{CH}), 39.1(\mathrm{CH}), 26.0\left(\mathrm{CH}_{2}\right), 25.1\left(\mathrm{CH}_{3}\right)$ and $24.8\left(\mathrm{CH}_{2}\right)$ ppm; IR (thin film): $v_{\max }$ 2924, 1775, 1700, 1607 and $1510 \mathrm{~cm}^{-1}$; EIMS (70 eV) m/z (\%) $297\left(\mathrm{M}^{+}, 100 \%\right), 185$ (76), 163 (24), 133 (25) and 105 (58); HRMS: calcd for $\mathrm{C}_{18} \mathrm{H}_{19} \mathrm{NO}_{3}\left[\mathrm{M}^{+}\right]$: 297.1365; found: 297.1364.

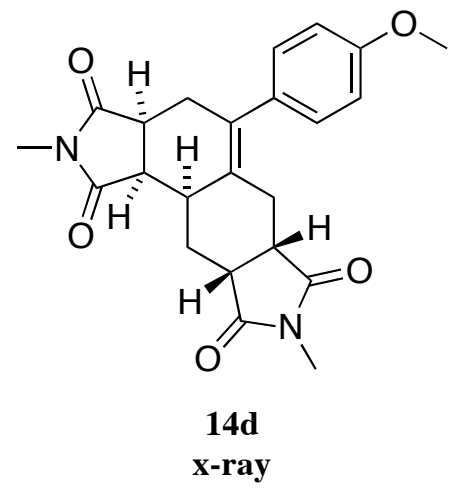

Bis-adduct 14d was isolated as colourless prisms, mp $215-216{ }^{\circ} \mathrm{C}$ $\left(\mathrm{CH}_{2} \mathrm{Cl}_{2}: 40-60{ }^{\circ} \mathrm{C}\right.$ petrol); $\mathrm{Rf} 0.10\left(95: 5 \mathrm{CH}_{2} \mathrm{Cl}_{2}: \mathrm{Et}_{2} \mathrm{O}\right) ;{ }^{1} \mathrm{H} \mathrm{NMR}(600$ $\left.\mathrm{MHz}, \mathrm{CDCl}_{3}\right) \delta 6.99(2 \mathrm{H}, \mathrm{d}, J=9.0 \mathrm{~Hz}), 6.86(2 \mathrm{H}, \mathrm{d}, J=9.0 \mathrm{~Hz})$, $3.78(3 \mathrm{H}, \mathrm{s}), 3.24(1 \mathrm{H}, \mathrm{m}), 3.16(1 \mathrm{H}, \mathrm{dd}, J=7.2 \mathrm{~Hz}), 3.01(4 \mathrm{H}, \mathrm{m})$, $3.00(3 \mathrm{H}, \mathrm{s}), 2.92(3 \mathrm{H}, \mathrm{s}), 2.83(1 \mathrm{H}, \mathrm{ddd}, J=13.8,13.8,5.4 \mathrm{~Hz}), 2.42$ $(2 \mathrm{H}, \mathrm{m})$ and $2.18(2 \mathrm{H}, \mathrm{m}) \mathrm{ppm} ;{ }^{13} \mathrm{C} \mathrm{NMR}\left(75 \mathrm{MHz}, \mathrm{CDCl}_{3}\right) \delta 180.1$ 
(C), 179.7 (C), 179.5 (C), 177.9 (C), 158.6 (C), 135.4 (C), 131.8 (C), 129.6 (C), $129.1(2 \times$ CH) 113.8 $(2 \times \mathrm{CH}), 55.3\left(\mathrm{CH}_{3}\right), 43.5(\mathrm{CH}), 40.4(\mathrm{CH}), 39.4(\mathrm{CH}), 39.0(\mathrm{CH}), 34.5(\mathrm{CH}), 32.1\left(\mathrm{CH}_{2}\right) 25.1\left(\mathrm{CH}_{3}\right)$, $25.0\left(\mathrm{CH}_{3}\right), 24.8\left(\mathrm{CH}_{2}\right)$ and $23.4\left(\mathrm{CH}_{2}\right)$ ppm; IR (thin film): $v_{\max } 2946,1772,1693,1607$ and $1512 \mathrm{~cm}^{-1}$; EIMS (70 eV) $m / z(\%) 408\left(\mathrm{M}^{+}, 100 \%\right), 322$ (13), 296 (12) and 112 (18); HRMS: calcd for $\mathrm{C}_{23} \mathrm{H}_{24} \mathrm{~N}_{2} \mathrm{O}_{5}$ $\left[\mathrm{M}^{+}\right]$: 408.1685; found: 408.1687; Found: C, 67.46; H, 5.89; N, 6.83. $\mathrm{C}_{23} \mathrm{H}_{24} \mathrm{~N}_{2} \mathrm{O}_{5}$ requires C, 67.63; $\mathrm{H}$, $5.92 ; \mathrm{N}, 6.86 \%$.

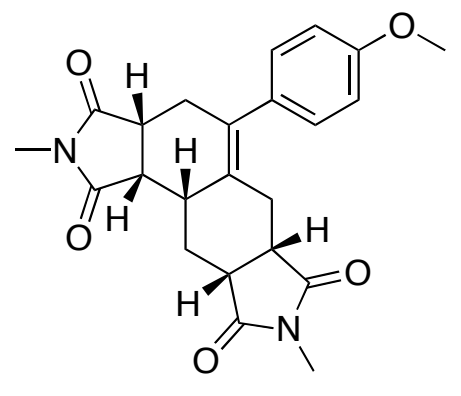

15d

Bis-adduct 15d was isolated as a colourless oil; Rf 0.10 (95:5 $\left.\mathrm{CH}_{2} \mathrm{Cl}_{2}: \mathrm{Et}_{2} \mathrm{O}\right) ;{ }^{1} \mathrm{H} \mathrm{NMR}\left(300 \mathrm{MHz}, \mathrm{CDCl}_{3}\right) \delta 6.94(2 \mathrm{H}, \mathrm{d}, J=9.0 \mathrm{~Hz})$, $6.85(2 \mathrm{H}, \mathrm{d}, J=9.0 \mathrm{~Hz}), 3.80(3 \mathrm{H}, \mathrm{s}), 3.24(1 \mathrm{H}, \mathrm{m}), 3.15(1 \mathrm{H}, \mathrm{m})$, $3.02(2 \mathrm{H}, \mathrm{m}), 2.99(3 \mathrm{H}, \mathrm{s}), 2.90(3 \mathrm{H}, \mathrm{s}), 2.80(2 \mathrm{H}, \mathrm{m}), 2.55(1 \mathrm{H}, \mathrm{m})$, $2.43(3 \mathrm{H}, \mathrm{m})$ and $1.95(1 \mathrm{H}, \mathrm{br} . \mathrm{dd}, J=12.6,12.6 \mathrm{~Hz}) \mathrm{ppm} ;{ }^{13} \mathrm{C} \mathrm{NMR}$ $\left(75 \mathrm{MHz}, \mathrm{CDCl}_{3}\right) \delta 179.4$ (C) 179.0 (C) 178.9 (C), 177.8 (C), 158.8 (C), 134.3 (C), 132.1 (C), $131.5(\mathrm{C}), 129.1(2 \times \mathrm{CH}), 114.0(2 \times \mathrm{CH})$, $55.4\left(\mathrm{CH}_{3}\right), 43.5(\mathrm{CH}), 40.7(\mathrm{CH}), 40.1(\mathrm{CH}), 39.6(\mathrm{CH}), 37.3(\mathrm{CH}), 31.8\left(\mathrm{CH}_{2}\right), 25.2\left(\mathrm{CH}_{2}\right), 25.0$ $\left(\mathrm{CH}_{2}\right)$ and $24.9\left(2 \times \mathrm{CH}_{3}\right) \mathrm{ppm}$; IR (thin film): $v_{\max }$ 2952, 1774, 1697, 1608 and $1511 \mathrm{~cm}^{-1}$; EIMS (70 eV) $m / z(\%) 408\left(\mathrm{M}^{+}, 100 \%\right), 297$ (13) and 108 (33); HRMS: calcd for $\mathrm{C}_{23} \mathrm{H}_{24} \mathrm{~N}_{2} \mathrm{O}_{5}\left[\mathrm{M}^{+}\right]$: 408.1685 ; found: 408.1684 .

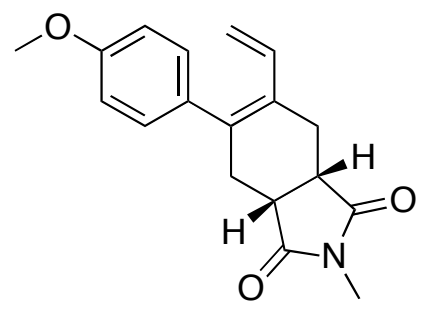

16d

Mono-adduct 16d was isolated as a colourless oil; Rf 0.43 (95:5 $\left.\mathrm{CH}_{2} \mathrm{Cl}_{2}: \mathrm{Et}_{2} \mathrm{O}\right) ;{ }^{1} \mathrm{H} \mathrm{NMR}\left(500 \mathrm{MHz}, \mathrm{CDCl}_{3}\right) \delta 7.06(2 \mathrm{H}, \mathrm{d}, J=9.0 \mathrm{~Hz})$, $6.85(2 \mathrm{H}, \mathrm{d}, J=9.0 \mathrm{~Hz}), 6.53(1 \mathrm{H}, \mathrm{dd}, J=17.0,11.0 \mathrm{~Hz}), 5.33(1 \mathrm{H}, \mathrm{d}, J$ $=17.0 \mathrm{~Hz}), 5.00(1 \mathrm{H}, \mathrm{d}, J=11.0 \mathrm{~Hz}), 3.80(3 \mathrm{H}, \mathrm{s}), 3.23(2 \mathrm{H}, \mathrm{m}), 3.11(1$ $\mathrm{H}, \mathrm{dd}, J=14.5,2.5 \mathrm{~Hz}), 2.97(1 \mathrm{H}, \mathrm{dd}, J=16.0,2.5 \mathrm{~Hz}), 2.94(3 \mathrm{H}, \mathrm{s})$, $2.66(1 \mathrm{H}, \mathrm{dd}, J=16.0,7.0 \mathrm{~Hz})$ and $2.32(1 \mathrm{H}, \mathrm{dd}, J=14.5,6.5 \mathrm{~Hz}) \mathrm{ppm}$;

${ }^{13} \mathrm{C}$ NMR $\left(75 \mathrm{MHz}, \mathrm{CDCl}_{3}\right) \delta 180.0$ (C) 179.6 (C) 158.8 (C), 137.1 (C), 134.5 (CH), 132.6 (C), 131.1(C), $130.2(2 \times \mathrm{CH}), 113.6(2 \times \mathrm{CH}), 113.4\left(\mathrm{CH}_{2}\right), 55.4\left(\mathrm{CH}_{3}\right), 40.0(2 \times \mathrm{CH}), 32.1\left(\mathrm{CH}_{2}\right), 25.1$ $\left(\mathrm{CH}_{3}\right)$ and $24.4\left(\mathrm{CH}_{2}\right)$ ppm; IR (thin film): $v_{\max } 2954,1775,1697,1607$ and $1510 \mathrm{~cm}^{-1}$; EIMS (70 eV) $m / z(\%) 297\left(\mathrm{M}^{+}, 100 \%\right), 282(25)$ and 266 (36); HRMS: calcd for $\mathrm{C}_{18} \mathrm{H}_{19} \mathrm{NO}_{3}\left[\mathrm{M}^{+}\right]$: 297.1365; found: 297.1365 . 


\section{Diels-Alder reaction of mono-adduct $16 \mathrm{~d}$ with $N$-methylmaleimide}

Use of five molar equivalents of $N$-methylmaleimide gave a $83 \%$ isolated yield of bis-adduct $\mathbf{1 7 d}$ and bis-adduct $\mathbf{1 8 d}$ in a 70:30 ratio.

Mono-adduct 16d (18 mg, $0.06 \mathrm{mmol}, 1$ equiv) and $N$-methylmaleimide (34 mg, $0.30 \mathrm{~mol}, 5$ equiv) were dissolved in $\mathrm{CDCl}_{3}(1.5 \mathrm{~mL})$ and the resulting solution was placed in a high-pressure reaction vessel for $40 \mathrm{~h}$ at $19 \mathrm{kbar}$ at room temperature. The solvent was removed under reduced pressure and the residue was purified by flash chromatography $\left(95: 5 \mathrm{CH}_{2} \mathrm{Cl}_{2}: \mathrm{Et}_{2} \mathrm{O}\right)$ to yield bisadducts $\mathbf{1 7 d}: \mathbf{1 8 d}$ 70:30 (21 $\mathrm{mg}, 83 \%)$ as a colourless oil. Bis-adducts $\mathbf{1 7 d}$ and $\mathbf{1 8 d}$ were further separated by normal phase HPLC (0.8:99.2 $\left.\mathrm{MeOH}: \mathrm{CHCl}_{3}\right)$.

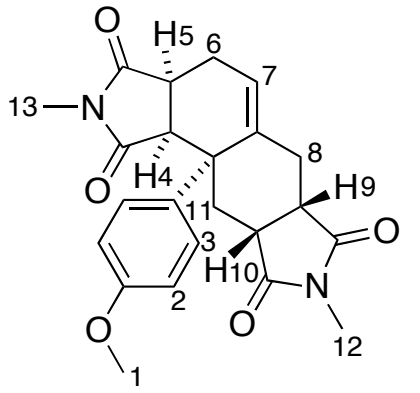

17d

Bis-adduct 17d was isolated as a colourless oil; $\operatorname{Rf} 0.07$ (95:5 $\left.\mathrm{CH}_{2} \mathrm{Cl}_{2}: \mathrm{Et}_{2} \mathrm{O}\right) ;{ }^{1} \mathrm{H} \mathrm{NMR}\left(600 \mathrm{MHz}, \mathrm{CDCl}_{3}\right) \delta 7.09(2 \mathrm{H}-3, \mathrm{~d}, J=9.0$ Hz), 6.77 (2 H-2, d, $J=9.0 \mathrm{~Hz}), 5.96(1 \mathrm{H}-7, \mathrm{dt}, J=7.5,3.0 \mathrm{~Hz}), 3.77$ (3 H-1, s), 3.59 (1 H-11 $\beta$, dd, $J=14.5,7.5 \mathrm{~Hz}), 3.43(1 \mathrm{H}-4, \mathrm{~d}, J=8.0$

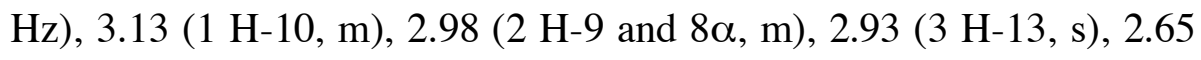

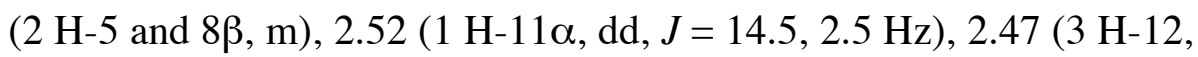
s), $2.27(1 \mathrm{H}-6 \beta, \mathrm{dd}, J=17.0,7.5 \mathrm{~Hz})$ and $1.99(1 \mathrm{H}-6 \alpha, \mathrm{m}) \mathrm{ppm} ;{ }^{13} \mathrm{C}$ NMR (125 MHz, $\mathrm{CDCl}_{3}$ ) $\delta 179.9$ (C) 179.8 (C) 178.7 (C), 177.6 (C), 158.5 (C), 138.6 (C), 131.0 (C), $128.4(2 \times \mathrm{CH}-3), 124.4(\mathrm{CH}-7), 113.8(2 \times \mathrm{CH}-2), 55.3\left(\mathrm{CH}_{3}-1\right), 51.9(\mathrm{CH}-4), 44.7(\mathrm{C}), 39.0(\mathrm{CH}-5)$, 38.1 (CH-9), 37.6 (CH-10), $31.8\left(\mathrm{CH}_{2}-11\right), 29.2\left(\mathrm{CH}_{2}-8\right), 25.7\left(\mathrm{CH}_{2}-6\right), 25.0\left(\mathrm{CH}_{3}-13\right)$ and $24.9\left(\mathrm{CH}_{3}-\right.$ 12) ppm; IR (thin film): $v_{\max } 2943,1772,1695,1607$ and $1510 \mathrm{~cm}^{-1}$; EIMS $(70 \mathrm{eV}) \mathrm{m} / z(\%) 408\left(\mathrm{M}^{+}\right.$, 68\%), 297 (100), 272 (39) and 108 (66); HRMS: calcd for $\mathrm{C}_{23} \mathrm{H}_{24} \mathrm{~N}_{2} \mathrm{O}_{5}\left[\mathrm{M}^{+}\right]$: 408.1685; found: 408.1685 .

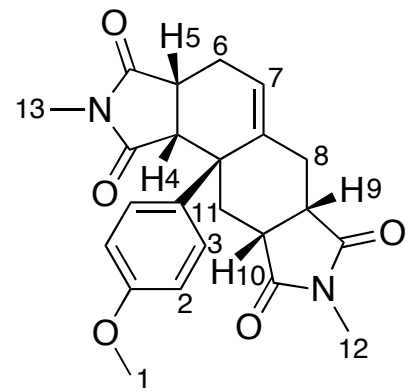

18d
Bis-adduct 18d was isolated as a colourless oil; $\operatorname{Rf} 0.07$ (95:5 $\mathrm{CH}_{2} \mathrm{Cl}_{2}: \mathrm{Et}_{2} \mathrm{O}$ ); ${ }^{1} \mathrm{H}$ NMR (800 MHz, $\mathrm{CDCl}_{3}$ ) $\delta 7.27$ (2 H-3, br. s), 6.87 (2 H-2, br. s), 5.95 (1 H-7, dt, $J=7.2,3.2 \mathrm{~Hz}), 3.80$ (3 H-1, s), 3.58 (1 $\mathrm{H}-4, \mathrm{~d}, J=8.0 \mathrm{~Hz}), 3.06(1 \mathrm{H}-8, \mathrm{dd}, J=13.6,13.6 \mathrm{~Hz}), 2.94(3 \mathrm{H}-12$, 
s), 2.92 (3 H-13, s), 2.87 (1 H-11, m), 2.83 (2 H-5 and 10, m), 2.37 (2 H-8 and 6ß, m), 2.27 (2 H-9 and $11, \mathrm{~m})$ and $1.95(1 \mathrm{H}-6 \alpha, \mathrm{m}) \mathrm{ppm} ;{ }^{13} \mathrm{C} \mathrm{NMR}\left(75 \mathrm{MHz}, \mathrm{CDCl}_{3}, 50{ }^{\circ} \mathrm{C}\right) \delta 179.6(\mathrm{C}) 179.5(\mathrm{C}) 179.1(\mathrm{C})$, 177.3 (C), 158.9 (C), 140.0 (C), 132.1 (C), 127.7 (2 × CH-3), $123.6(\mathrm{CH}-7), 114.7$ (2 × CH-2), 55.5 $\left(\mathrm{CH}_{3}-1\right)$, 50.4 (CH-4), $46.0(\mathrm{C}), 39.7$ (CH-10), 38.0 (CH-5), $37.3(\mathrm{CH}-9), 33.0\left(\mathrm{CH}_{2}-8\right), 30.7\left(\mathrm{CH}_{2}-11\right)$, $25.8\left(\mathrm{CH}_{2}-6\right), 25.0\left(\mathrm{CH}_{3}-12\right)$ and $24.8\left(\mathrm{CH}_{3}-13\right) \mathrm{ppm}$; IR (thin film): $v_{\max } 2950,1772,1698,1606$ and $1510 \mathrm{~cm}^{-1}$; EIMS (70 eV) m/z (\%) $408\left(\mathrm{M}^{+}, 72 \%\right), 297$ (100), 282 (25) and 108 (54); HRMS: calcd for $\mathrm{C}_{23} \mathrm{H}_{24} \mathrm{~N}_{2} \mathrm{O}_{5}\left[\mathrm{M}^{+}\right]:$408.1685; found: 408.1685 .

Diels-Alder reaction of 2-(4-nitrophenyl)[3]dendralene $\quad 3 e \quad$ with $N$-methylmaleimide

Use of five molar equivalents of $N$-methylmaleimide gave a $94 \%$ isolated yield of bis-adduct 14e, bisadduct 15e and mono-adduct $\mathbf{1 6 e}$ in a 68:5:27 ratio.

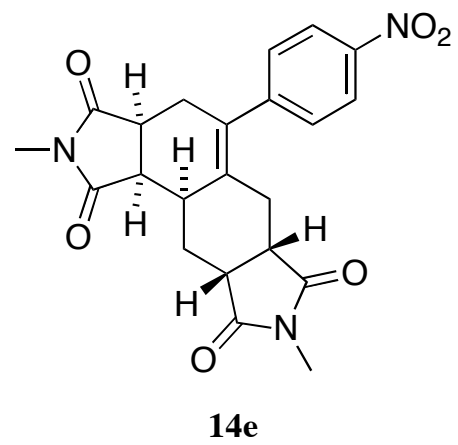

Bis-adduct 14e was isolated as a colourless prisms, mp $236-237$ ${ }^{\circ} \mathrm{C}\left(\mathrm{CH}_{2} \mathrm{Cl}_{2}\right.$ :hexane); Rf 0.11 (50:50 EtOAc:hexane); ${ }^{1} \mathrm{H}$ NMR (300 $\left.\mathrm{MHz}, \mathrm{CDCl}_{3}\right) \delta 8.71-8.24(2 \mathrm{H}, \mathrm{m}), 7.24-7.31(2 \mathrm{H}, \mathrm{m}), 3.11-3.33$ (4 H, m), 2.91-3.03 (2 H, m), $3.02(3 \mathrm{H}, \mathrm{s}), 2.96(3 \mathrm{H}, \mathrm{s}), 2.83(1 \mathrm{H}$, ddd, $J=14.0,5.8 \mathrm{~Hz}), 2.42-2.54,(2 \mathrm{H}, \mathrm{m})$ and $2.14-2.28(2 \mathrm{H}, \mathrm{m})$ ppm. ${ }^{13} \mathrm{C} \mathrm{NMR}\left(75 \mathrm{MHz}, \mathrm{CDCl}_{3}\right) \delta 179.7$ (C), $179.4(\mathrm{C}), 179.1(\mathrm{C})$, 177.3 (C), 146.7 (C), 146.0 (C), 134.0 (C), 133.5 (C), 128.9 (CH), $123.8(\mathrm{CH}), 43.1(\mathrm{CH}), 40.2(\mathrm{CH}), 39.0(\mathrm{CH}), 38.7(\mathrm{CH}), 34.6(\mathrm{CH}), 31.5\left(\mathrm{CH}_{2}\right), 29.6\left(\mathrm{CH}_{2}\right), 25.1$ $\left(\mathrm{CH}_{3}\right), 25.0\left(\mathrm{CH}_{3}\right), 24.8\left(\mathrm{CH}_{2}\right)$ and $23.2\left(\mathrm{CH}_{2}\right)$ ppm; IR (thin film): $v_{\max }$ 2946, 1773, 1699 and 1517 $\mathrm{cm}^{-1}$; EIMS (70 eV) m/z (\%) $423\left(\mathrm{M}^{+}, 100 \%\right), 406$ (89) and 112 (88); HRMS: calcd for $\mathrm{C}_{22} \mathrm{H}_{21} \mathrm{~N}_{3} \mathrm{O}_{6}$ $\left[\mathrm{M}^{+}\right]$: 432.1430; found: 423.1427 .

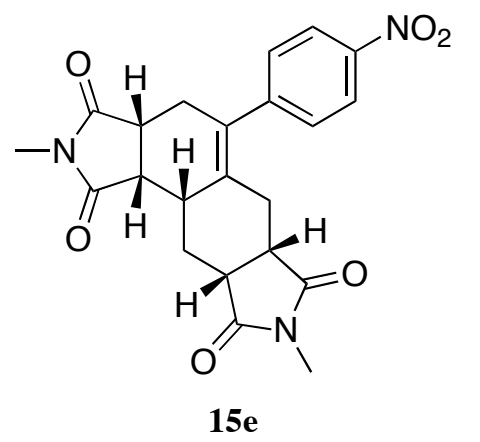

Bis-adduct 15e was isolated as a colourless oil; Rf 0.05 (50:50 EtOAc:hexane); ${ }^{1} \mathrm{H}$ NMR (300 MHz, $\left.\mathrm{CDCl}_{3}\right) \delta 8.17-8.23(2 \mathrm{H}, \mathrm{m})$, 7.14-7.21 (2 H, m), 3.31 ( $1 \mathrm{H}, \mathrm{dd}, J=8.7,1.4 \mathrm{~Hz}), 3.23(1 \mathrm{H}, \mathrm{dd}, J=$ 8.7, 4.8 Hz), 2.75- $3.07(10 \mathrm{H}, \mathrm{m}), 2.62(1 \mathrm{H}, \mathrm{ddd}, J=14.8,6.3,4.0$ 
$\mathrm{Hz}), 2.44-2.54(3 \mathrm{H}, \mathrm{m})$ and $2.01(1 \mathrm{H}$, br. dd, $J=13.5,13.5 \mathrm{~Hz}) \mathrm{ppm} .{ }^{13} \mathrm{C} \mathrm{NMR}\left(75 \mathrm{MHz}, \mathrm{CDCl}_{3}\right) \delta$ 178.9 (C), 178.4 (C), 178.4 (C), 177.1 (C), 146.8 (C), 146.2 (C), 135.6 (C), 133.0 (C), 128.8 (C), 124.0 (C), $43.0(\mathrm{CH}), 40.5(\mathrm{CH}), 39.1(\mathrm{CH}), 37.4(\mathrm{CH}), 31.2\left(\mathrm{CH}_{2}\right), 25.1\left(\mathrm{CH}_{2}\right), 25.0\left(\mathrm{CH}_{3}\right), 24.9\left(\mathrm{CH}_{2}\right)$ and $24.8\left(\mathrm{CH}_{2}\right) \mathrm{ppm}$; IR (thin film): $v_{\max } 2915,1695$ and $1516 \mathrm{~cm}^{-1}$; ESIMS (70 eV) $\mathrm{m} / z(\%) 445\left(\mathrm{M}^{+}+\mathrm{Na}^{+}\right.$, 17\%), 423 (4), 366 (22), 365 (100) and 337 (47); HRMS: calcd for $\mathrm{C}_{22} \mathrm{H}_{21} \mathrm{~N}_{3} \mathrm{O}_{6} \mathrm{Na}\left[\mathrm{M}^{+}+\mathrm{Na}\right]$ : 446.1328; found: 446.1328 .

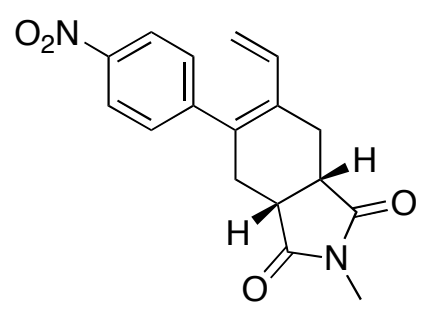

$16 \mathbf{e}$

Mono-adduct 16e was isolated as a colourless oil; Rf 0.27 (30:70 EtOAc:40 - $60{ }^{\circ} \mathrm{C}$ petrol); ${ }^{1} \mathrm{H}$ NMR $\left(300 \mathrm{MHz}, \mathrm{CDCl}_{3}\right) \delta 8.16-8.22(2 \mathrm{H}$, m), 7.26-7.32 (2 H, m), $6.42(1 \mathrm{H}, \mathrm{dd}, J=17.5,11.0 \mathrm{~Hz}), 5.46(1 \mathrm{H}, \mathrm{d}, J$ $=17.5 \mathrm{~Hz}), 5.13(1 \mathrm{H}, J=11.0 \mathrm{~Hz}), 3.23-3.36(2 \mathrm{H}, \mathrm{m}), 3.21(1 \mathrm{H}, \mathrm{dd}, J$ = 14.7, $2.2 \mathrm{~Hz}), 3.05(1 \mathrm{H}, \mathrm{dd}, J=15.7 \mathrm{~Hz}), 2.96(3 \mathrm{H}, \mathrm{s}), 2.70(1 \mathrm{H}, \mathrm{dd}$, $J=15.4,5.8 \mathrm{~Hz})$ and $2.35(1 \mathrm{H}, \mathrm{dd}, J=15.4,6.6 \mathrm{~Hz}) \mathrm{ppm} .{ }^{13} \mathrm{C} \mathrm{NMR}(75$

$\mathrm{MHz}, \mathrm{CDCl}_{3}$ ) $\delta 179.6$ (C) 179.1 (C) 146.9 (C), 146.7 (C), 135.1 (C), 134.3 (C), 133.1 (CH), 129.8 $(\mathrm{CH}), 123.5(\mathrm{CH}), 116.1\left(\mathrm{CH}_{2}\right), 39.8(\mathrm{CH}), 39.5(\mathrm{CH}), 31.5\left(\mathrm{CH}_{2}\right), 25.1\left(\mathrm{CH}_{3}\right)$ and $24.5\left(\mathrm{CH}_{2}\right)$ ppm; IR (thin film): $v_{\max } 2917,1776,1698$ and $1618 \mathrm{~cm}^{-1}$; EIMS (70 eV) $\mathrm{m} / z(\%) 312\left(\mathrm{M}^{+}, 30 \%\right), 311$ (22) and 295 (100); HRMS: calcd for $\mathrm{C}_{17} \mathrm{H}_{16} \mathrm{~N}_{2} \mathrm{O}_{4}\left[\mathrm{M}^{+}\right]$: 312.1110; found: 312.1109 .

\section{Diels-Alder reaction of 2-ethoxy[3]dendralene 3f with $N$-methylmaleimide}

Use of five molar equivalents of $N$-methylmaleimide gave a $41 \%$ isolated yield of bis-adduct $\mathbf{1 4 f}$, bisadduct 15f and mono-adduct $\mathbf{1 6 f}$ in a 81:9:10 ratio.

A mixture of 2-ethoxy[3]dendralene $3 \mathbf{f}(69 \mathrm{mg}, 0.56 \mathrm{mmol}, 1$ equiv), $N$-methylmaleimide (200 mg, 1.8 mmol, 3.2 equiv), calcium hydride $(10 \mathrm{mg})$ and anhydrous $\mathrm{CH}_{2} \mathrm{Cl}_{2}(5 \mathrm{~mL})$ was stirred at room temperature for 18 hours. The reaction mixture was filtered and concentrated under reduced pressure $(0$ ${ }^{\circ} \mathrm{C}, 30 \mathrm{mmHg}$ ) to give an oil which was subjected to radial chromatography. Elution with $30-50 \%$ EtOAc/hexanes gave mono-adduct $\mathbf{1 6 f}$ as a colourless oil $(5 \mathrm{mg}, 4 \%)$, bis-adduct $\mathbf{1 4 f}$ as a colourless solid (64 mg, 33\%), bis-adduct $\mathbf{1 5 f}$ as a colourless oil (8 $\mathrm{mg}, 4 \%$ ). 


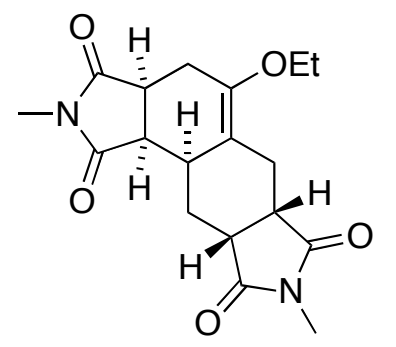

$14 f$

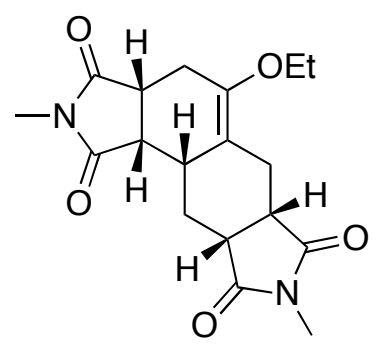

$15 f$

The compound had identical ${ }^{1} \mathrm{H}$ and ${ }^{13} \mathrm{C}$ n.m.r spectra to those reported for 14f. ${ }^{9}$ Bis-adduct 14f was isolated as colourless prisms, mp $135-138{ }^{\circ} \mathrm{C}$ (EtOAc:hexane), Lit. ${ }^{9} 113-116^{\circ} \mathrm{C}$; Rf 0.21 (30:70 EtOAc:hexane).

Bis-adduct 15f was isolated as a colourless oil; Rf 0.12 (30:70 EtOAc:40 $-60{ }^{\circ} \mathrm{C}$ petrol); ${ }^{1} \mathrm{H}$ NMR $\left(300 \mathrm{MHz}, \mathrm{CDCl}_{3}\right) \delta 3.72(1 \mathrm{H}, \mathrm{dq}, J=9.7,7.0$ $\mathrm{Hz}), 3.56(1 \mathrm{H}, \mathrm{dq}, J=9.7,7.0 \mathrm{~Hz}), 3.24(1 \mathrm{H}, \mathrm{dd}, J=14.8,6.7 \mathrm{~Hz}), 3.19$ $(1 \mathrm{H}, \mathrm{dd}, J=17.0,8.2 \mathrm{~Hz}), 3.06(1 \mathrm{H}$, br. d, $J=8.6 \mathrm{~Hz}), 2.99(3 \mathrm{H}, \mathrm{s}), 2.93$ $(3 \mathrm{H}, \mathrm{s}), 2.62-2.88(3 \mathrm{H}, \mathrm{m}), 2.20-2.44(4 \mathrm{H}, \mathrm{m}), 1.74(1 \mathrm{H}$, br. dd, $J=$ $11.5,11.5 \mathrm{~Hz})$ and $1.16(3 \mathrm{H}, \mathrm{t}, J=7.0 \mathrm{~Hz}) \mathrm{ppm} ;{ }^{13} \mathrm{C} \mathrm{NMR}(125 \mathrm{MHz}$, $\left.\mathrm{CDCl}_{3}\right) \delta 179.2(\mathrm{C}), 179.2(\mathrm{C}), 179.0(\mathrm{C}), 177.3(\mathrm{C}), 147.3(\mathrm{C}), 114.6(\mathrm{C})$,

$64.7(\mathrm{CH} 2), 43.3(\mathrm{CH}), 40.5(\mathrm{CH}), 40.1(\mathrm{CH}), 39.0(\mathrm{CH}), 35.7(\mathrm{CH}), 29.7\left(\mathrm{CH}_{2}\right), 25.5\left(\mathrm{CH}_{2}\right), 25.0$ $\left(\mathrm{CH}_{3}\right), 25.8\left(\mathrm{CH}_{3}\right), 20.7\left(\mathrm{CH}_{2}\right)$ and $15.1\left(\mathrm{CH}_{3}\right)$ ppm; IR (thin film): $v_{\max } 2975,1774$ and $1696 \mathrm{~cm}^{-1}$; EIMS (70 eV) $\mathrm{m} / \mathrm{z}(\%) 346\left([M]^{+}, 59 \%\right), 333$ (11) and 317 (100); HRMS: calc for $\mathrm{C}_{30} \mathrm{H}_{29} \mathrm{~N}_{3} \mathrm{O}_{6}[M]^{+}$: 346.1529; found: 346.1530 .

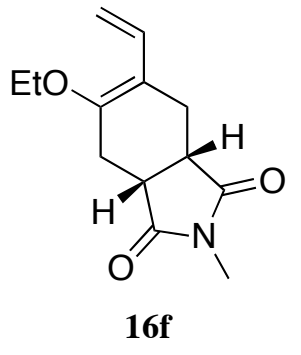

Mono-adduct 16f was isolated as a colourless oil; Rf 0.41 (30:70 EtOAc: $40-60{ }^{\circ} \mathrm{C}$ petrol); ${ }^{1} \mathrm{H}$ NMR $\left(300 \mathrm{MHz}, \mathrm{CDCl}_{3}\right) \delta 6.82(1 \mathrm{H}, \mathrm{dd}, J$ = 17.6, $11.0 \mathrm{~Hz}), 5.13(1 \mathrm{H}, \mathrm{d}, J=17.6 \mathrm{~Hz}), 4.93(1 \mathrm{H}, \mathrm{d}, J=11.0 \mathrm{~Hz})$, $3.83(1 \mathrm{H}, \mathrm{dq}, J=9.6,7.0 \mathrm{~Hz}), 3.66(1 \mathrm{H}, \mathrm{dq}, J=9.6,7.0 \mathrm{~Hz}), 3.07-3.22$ $(2 \mathrm{H}, \mathrm{m}), 2.94(3 \mathrm{H}, \mathrm{s}), 2.93(1 \mathrm{H}, \mathrm{dd}, J=15.2,2.7 \mathrm{~Hz}), 2.83(1 \mathrm{H}, \mathrm{dd}, J=$ $15.8,2.2 \mathrm{~Hz}) 2.42(1 \mathrm{H}, \mathrm{dd}, J=15.6,6.9 \mathrm{~Hz}), 2.24(1 \mathrm{H}, \mathrm{dd}, J=15.1,3.0$ $\mathrm{Hz})$ and $1.20(3 \mathrm{H}, \mathrm{t}, J=7.0 \mathrm{~Hz}) \mathrm{ppm} .{ }^{13} \mathrm{C} \mathrm{NMR}\left(75 \mathrm{MHz}, \mathrm{CDCl}_{3}\right) \delta 179.7(\mathrm{C}), 179.4(\mathrm{C}), 149.8(\mathrm{C})$, $130.0(\mathrm{CH}), 115.9\left(\mathrm{CH}_{2}\right), 110.6(\mathrm{C}), 64.6\left(\mathrm{CH}_{2}\right), 39.9(\mathrm{CH}), 39.1(\mathrm{CH}), 25.1\left(\mathrm{CH}_{2}\right), 24.9\left(\mathrm{CH}_{2}\right), 22.6$ $\left(\mathrm{CH}_{3}\right)$ and $15.1\left(\mathrm{CH}_{3}\right)$ ppm; IR (thin film): $v_{\max } 2925$ and $1699 \mathrm{~cm}^{-1}$; EIMS $(70 \mathrm{eV}) \mathrm{m} / z(\%) 235\left(\mathrm{M}^{+}\right.$, $100 \%$ ) and 220 (16); HRMS: calcd for $\mathrm{C}_{13} \mathrm{H}_{17} \mathrm{NO}_{3}\left[\mathrm{M}^{+}\right]$: 235.1208; found: 235.1210. 


\section{Diels-Alder reaction of 2-carbomethoxy[3]dendralene $3 \mathrm{~g}$ with $\mathrm{N}$-methylmaleimide}

Use of five molar equivalents of $N$-methylmaleimide gave a $66 \%$ isolated yield of bis-adduct $\mathbf{1 4 g}$, bisadduct $\mathbf{1 5 g}$ and mono-adduct $\mathbf{1 6 g}$ in a 68:8:24 ratio.

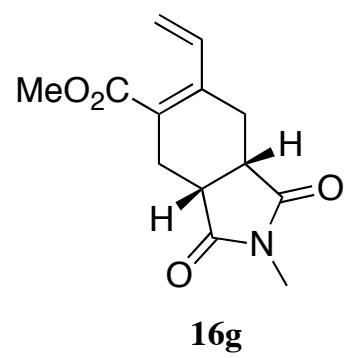

Mono-adduct 16g was isolated as a colourless oil; Rf 0.27 (30:70 EtOAc:40 - $60{ }^{\circ} \mathrm{C}$ petrol); ${ }^{1} \mathrm{H}$ NMR $\left(300 \mathrm{MHz}, \mathrm{CDCl}_{3}\right) \delta 7.46(1 \mathrm{H}, \mathrm{dd}, J$ = 17.5, $11.1 \mathrm{~Hz}), 5.63(1 \mathrm{H}, \mathrm{d}, J=17.5 \mathrm{~Hz}), 5.38(1 \mathrm{H}, \mathrm{d}, J=11.1 \mathrm{~Hz})$, $3.75(3 \mathrm{H}, \mathrm{s}), 3.10-3.22$ (3 H, m), 3.01-3.10 (1 H, m), 2.93 (3 H, s), 2.48$2.59(1 \mathrm{H}, \mathrm{m})$ and 2.35-2.45 $(1 \mathrm{H}, \mathrm{m}) \mathrm{ppm} .{ }^{13} \mathrm{C} \mathrm{NMR}\left(75 \mathrm{MHz}, \mathrm{CDCl}_{3}\right) \delta$ $178.9(\mathrm{C}), 178.7$ (C), 166.5 (C), 145.6 (C), $133.4(\mathrm{CH}), 125.8$ (C), 119.4 $\left(\mathrm{CH}_{2}\right)$, $51.9\left(\mathrm{CH}_{3}\right), 39.4(\mathrm{CH}), 39.0(\mathrm{CH}), 26.1\left(\mathrm{CH}_{2}\right), 25.2\left(\mathrm{CH}_{2}\right)$ and $25.1\left(\mathrm{CH}_{3}\right)$ ppm; IR (thin film): $v_{\max } 2952$ and $1699 \mathrm{~cm}^{-1}$; EIMS (70 eV) m/z (\%) $249\left(\mathrm{M}^{+}, 67 \%\right), 217$ (27), 132 (53), 105 (93) and 104 (100); HRMS: calcd for $\mathrm{C}_{13} \mathrm{H}_{15} \mathrm{NO}_{4}\left[\mathrm{M}^{+}\right]$: 249.1001; found: 249.1007 .

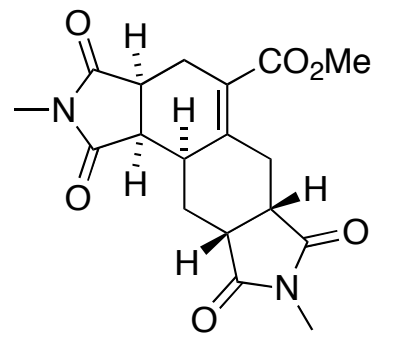

$14 \mathrm{~g}$

Bis-adduct 14g was recrystallised to give colourless prisms, mp 233 - 237 ${ }^{\circ} \mathrm{C}\left(\mathrm{CH}_{2} \mathrm{Cl}_{2}\right.$ :hexane); Rf 0.14 (50:50 EtOAc:hexane); ${ }^{1} \mathrm{H}$ NMR (300 MHz, $\left.\mathrm{CDCl}_{3}\right) \delta 4.12(1 \mathrm{H}, \mathrm{dd}, J=15.7,2.1 \mathrm{~Hz}), 3.73(3 \mathrm{H}, \mathrm{s}), 3.32(1 \mathrm{H}, \mathrm{d}, J=$ $15.5 \mathrm{~Hz}), 3.09-3.28(3 \mathrm{H}, \mathrm{m}), 3.05(1 \mathrm{H}, \mathrm{dd}, J=8.9,5.5 \mathrm{~Hz}), 2.93(3 \mathrm{H}, \mathrm{s})$, $2.88(3 \mathrm{H}, \mathrm{s}), 2.81(1 \mathrm{H}, \mathrm{dd}, J=13.0,5.2 \mathrm{~Hz}), 2.41(1 \mathrm{H}, \mathrm{ddd}, J=14.4,4.8$, $2.4 \mathrm{~Hz}) 2.26-2.38(1 \mathrm{H} \mathrm{m})$ and 1.81-1.95 (2 H, m) ppm; ${ }^{13} \mathrm{C} \mathrm{NMR}(75 \mathrm{MHz}$, $\left.\mathrm{CDCl}_{3}\right) \delta 179.7(\mathrm{C}), 178.7(\mathrm{C}), 178.3$ (C), $177.1(\mathrm{C}), 148.8$ (C), 126.0 (C), $137.2(\mathrm{C}), 134.1(\mathrm{C}), 131.7(\mathrm{C}), 129.5(\mathrm{CH}), 129.0(\mathrm{CH}), 126.3(\mathrm{CH}), 53.4(\mathrm{CH}), 45.1(\mathrm{CH}), 43.9(\mathrm{C})$, $40.2(\mathrm{CH}), 39.5(\mathrm{CH}), 39.3(\mathrm{CH}), 38.4(\mathrm{CH}), 37.8(\mathrm{CH}), 36.7(\mathrm{CH}), 32.9\left(\mathrm{CH}_{2}\right), 32.6\left(\mathrm{CH}_{2}\right), 28.3$ $\left(\mathrm{CH}_{2}\right), 26.2\left(\mathrm{CH}_{2}\right), 25.4\left(\mathrm{CH}_{3}\right), 25.2\left(\mathrm{CH}_{3}\right)$ and $24.9\left(\mathrm{CH}_{2}\right)$ ppm; IR $(\mathrm{KBr} \operatorname{disc}) v=2952,1774$ and 1693 $\mathrm{cm}^{-1}$; EIMS (70 eV) m/z (\%) $360\left(\mathrm{M}^{+}, 2 \%\right), 329$ (30), 328 (100) and 300 (30); HRMS: calc for $\mathrm{C}_{18} \mathrm{H}_{20} \mathrm{~N}_{2} \mathrm{O}_{6}[M]^{+}: 360.1321$; found 360.1323. 


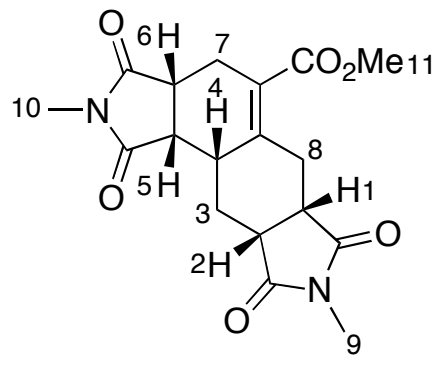

$15 \mathrm{~g}$

Bis-adduct 15g was isolated as a colourless oil; Rf $0.05(50: 50$ EtOAc:hexane); ${ }^{1} \mathrm{H}$ NMR (800 MHz, $\left.\mathrm{CDCl}_{3}\right) \delta 4.07(1 \mathrm{H}-8 \alpha, \mathrm{dd}, J=$ 15.2, $6.4 \mathrm{~Hz}$ ), 3.76 (3 H-11, s), 3.39 (1 H-7 , dd, $J=16.0,1.6 \mathrm{~Hz}$ ), 3.21 ( $1 \mathrm{H}-6$, ddd, $J=8.9,7.1,1.4 \mathrm{~Hz}), 3.14(1 \mathrm{H}-5$, dd, $J=8.8,6.4$ $\mathrm{Hz}), 3.02$ (3 H-9, s), 2.88 (3 H-10, s), 2.81 (2 H-1 and 2, m), 2.49 (1 H-3 $\beta$, dd, $J=26.7,12.8 \mathrm{~Hz}), 2.42(1 \mathrm{H}-3 \alpha, \mathrm{m}) 2.35(1 \mathrm{H}-4, \mathrm{~m}), 2.22$ $(1 \mathrm{H}-7 \beta, \mathrm{m})$ and $2.00(1 \mathrm{H}-8 \beta$, dd, $J=14.7,14.7 \mathrm{~Hz}) \mathrm{ppm} .{ }^{13} \mathrm{C}$ NMR $\left(75 \mathrm{MHz}, \mathrm{CDCl}_{3}\right) \delta 178.4(\mathrm{C}), 178.3(\mathrm{C}), 176.8(\mathrm{C}), 165.9(\mathrm{C}), 149.6(\mathrm{C}), 125.3(\mathrm{C}), 52.0\left(\mathrm{CH}_{3}-11\right)$, 42.7 (CH-5), 39.7 (CH-6), 39.5 (CH-2), 38.3 (CH-4), 38.2 (CH-1), $26.1\left(\mathrm{CH}_{2}-7\right), 25.4\left(\mathrm{CH}_{2}-8\right), 25.1$ $\left(\mathrm{CH}_{3}-9\right), 24.9\left(\mathrm{CH}_{3}-10\right)$ and $24.6\left(\mathrm{CH}_{2}-3\right) \mathrm{ppm}$; IR (thin film): $v_{\max } 2919$ and $1697 \mathrm{~cm}^{-1}$; EIMS (70 eV) $m / z(\%) 360\left(\mathrm{M}^{+}, 4 \%\right), 329$ (26), 328 (100) and 243 (35); HRMS: calcd for $\mathrm{C}_{18} \mathrm{H}_{20} \mathrm{~N}_{2} \mathrm{O}_{6}$ [ $\left.\mathrm{M}^{+}\right]: 360.1321$; found: 360.1333 .

\section{Diels-Alder reaction of [3]dendralene 1 with $N$-methylmaleimide}

Use of five molar equivalents of $N$-methylmaleimide gave a $66 \%$ isolated yield of bis-adduct $14 \mathbf{h}$ and bis-adduct $\mathbf{1 5 h}$ in a 90:10 ratio.

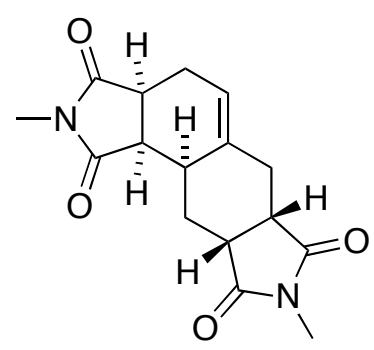

14h

Bis-adduct 14h was isolated as a colourless oil. $\operatorname{Rf}$ 0.13 (95:5 $\left.\mathrm{CH}_{2} \mathrm{Cl}_{2}: \mathrm{Et}_{2} \mathrm{O}\right) ;{ }^{1} \mathrm{H}$ NMR $\left(300 \mathrm{MHz}, \mathrm{CDCl}_{3}\right) \delta 5.66(1 \mathrm{H}, \mathrm{m}), 3.23(1 \mathrm{H}, \mathrm{m})$, $3.08(3 \mathrm{H}, \mathrm{m}), 2.95(3 \mathrm{H}, \mathrm{s}), 2.91(3 \mathrm{H}, \mathrm{s}), 2.69(3 \mathrm{H}, \mathrm{m}), 2.40(2 \mathrm{H}, \mathrm{m})$ and $2.05(2 \mathrm{H}, \mathrm{m}) \mathrm{ppm} ;{ }^{13} \mathrm{C} \mathrm{NMR}\left(75 \mathrm{MHz}, \mathrm{CDCl}_{3}\right) \delta 180.0(\mathrm{C}), 179.6(\mathrm{C})$, $179.5(\mathrm{C}), 177.8(\mathrm{C}), 136.6(\mathrm{C}), 122.8(\mathrm{CH}), 43.2(\mathrm{CH}), 40.1(\mathrm{CH}), 39.3(2$ $\times \mathrm{CH}), 33.1(\mathrm{CH}), 29.0\left(\mathrm{CH}_{2}\right), 25.0\left(\mathrm{CH}_{3}\right), 24.9\left(\mathrm{CH}_{3}\right), 24.7\left(\mathrm{CH}_{2}\right)$ and 23.3 $\left(\mathrm{CH}_{2}\right)$ ppm; IR (thin film): $v_{\max }$ 2948, 1772 and $1694 \mathrm{~cm}^{-1}$; EIMS (70 eV) $m / z(\%) 303\left(\mathrm{M}^{+1}, 64 \%\right), 302\left(\mathrm{M}^{+}, 100\right), 287$ (27) and 217 (44); HRMS: calcd for $\mathrm{C}_{16} \mathrm{H}_{18} \mathrm{~N}_{2} \mathrm{O}_{4}\left[\mathrm{M}^{+}\right]$: 302.1267 ; found 302.1266 . 


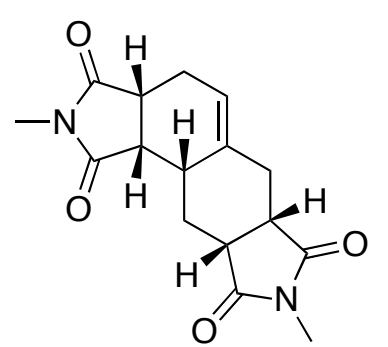

15h

Bis-adduct $15 \mathbf{h}$ was isolated as a colourless oil; $\mathrm{Rf}$ 0.13 (95:5 $\left.\mathrm{CH}_{2} \mathrm{Cl}_{2}: \mathrm{Et}_{2} \mathrm{O}\right) ;{ }^{1} \mathrm{H} \mathrm{NMR}\left(500 \mathrm{MHz}, \mathrm{CDCl}_{3}\right) \delta 5.74(1 \mathrm{H}, \mathrm{m}), 3.14(2 \mathrm{H}, \mathrm{m})$, $3.00(3 \mathrm{H}, \mathrm{s}), 2.95(2 \mathrm{H}, \mathrm{m}), 2.90(3 \mathrm{H}, \mathrm{s}), 2.84$ (1 H, m), 2.75 (1 H, dd, $J=$ 14.5, $7.0 \mathrm{~Hz}), 2.67(1 \mathrm{H}, \mathrm{m}), 2.36(2 \mathrm{H}, \mathrm{m}), 2.32(1 \mathrm{H}, \mathrm{m})$ and $2.15(1 \mathrm{H}, \mathrm{m})$ ppm; ${ }^{13} \mathrm{C}$ NMR (125 MHz, $\left.\mathrm{CDCl}_{3}\right) \delta 179.6$ (C), 179.1 (C), 178.9 (C), 177.6 (C), $138.4(\mathrm{C}), 121.6(\mathrm{CH}), 43.4(\mathrm{CH}), 40.2(\mathrm{CH}), 40.2(\mathrm{CH}), 39.3(\mathrm{CH})$, $35.9(\mathrm{CH}), 28.8\left(\mathrm{CH}_{2}\right), 25.0\left(\mathrm{CH}_{3}\right), 24.9\left(\mathrm{CH}_{3}\right), 24.8\left(\mathrm{CH}_{2}\right)$ and $24.8\left(\mathrm{CH}_{2}\right)$ ppm; IR (thin film): $v_{\max } 2925,1773,1694$ and $1435 \mathrm{~cm}^{-1}$; EIMS (70 eV) $\mathrm{m} / z$ (\%) $302\left(\mathrm{M}^{+}, 81 \%\right), 217$ (15), 191 (45) and 57 (100); HRMS: calcd for $\mathrm{C}_{16} \mathrm{H}_{18} \mathrm{~N}_{2} \mathrm{O}_{4}\left[\mathrm{M}^{+}\right]$: 302.1267; found: 302.1253. 


\section{Lewis Acid promoted Cycloaddition Reactions}

\section{Treatment of 2-Phenyl[3]dendralene 3b with $N$-Methylmaleimide-Ethylaluminium Dichloride Complex (1:1)}

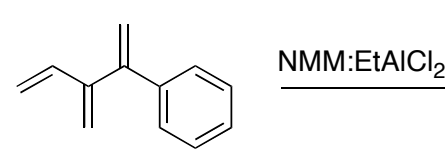

3b

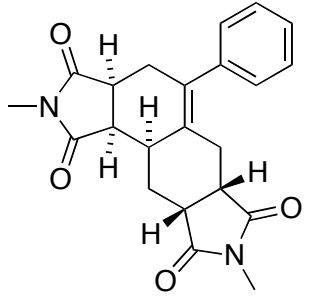

14b

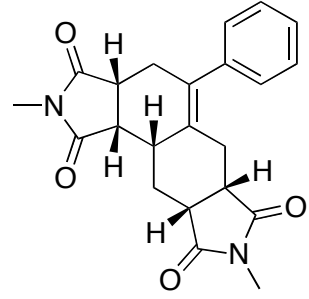

$15 b$

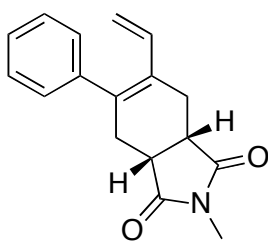

16b

Note: Commercially available N-methylmaleimide must be recrystallised from diethyl ether prior to use.

Ethylaluminium dichloride $(1.0 \mathrm{~mL}$ of a $1 \mathrm{M}$ solution in hexanes, $1.0 \mathrm{mmol}, 4.5$ equiv) was added dropwise to a stirred solution of $N$-methylmaleimide (116 mg, $1.04 \mathrm{mmol}, 4.64$ equiv) in anhydrous toluene $(0.6 \mathrm{~mL})$ at $-78{ }^{\circ} \mathrm{C}$ under nitrogen. The clear solution immediately turned deep red. After stirring for 15 minutes at $-78{ }^{\circ} \mathrm{C}$, a solution of 2-phenyl[3]dendralene $\mathbf{3 b}$ (35 $\mathrm{mg}, 0.22 \mathrm{mmol}, 1$ equiv) in toluene $(0.3 \mathrm{~mL})$ was added. The mixture was stirred at $-78{ }^{\circ} \mathrm{C}$ for one hour, then allowed to warm to room temperature of 15 minutes (during which time the red colour faded) and cooled again to -78 ${ }^{\circ} \mathrm{C}$. Water $(10 \mathrm{~mL})$ was added to the reaction and the mixture was allowed to warm to room temperature. The aqueous layer was acidified with $1 \mathrm{M} \mathrm{HCl}$ (aq.) and extracted with $\mathrm{CH}_{2} \mathrm{Cl}_{2}(10 \mathrm{~mL}$ ). The organic phase was dried over anhydrous magnesium sulphate and concentrated under reduced pressure $(20 \mathrm{mmHg})$ to give an oily solid. The solid was subjected to radial chromatography $(30-50 \%$ EtOAc $/ 40-60{ }^{\circ} \mathrm{C}$ petrol) to give the mono-adduct $\mathbf{1 6 b}(9 \mathrm{mg}, 15 \%)$, the bis-adduct $\mathbf{1 4 b}(15 \mathrm{mg}, 17 \%)$ and bis-adduct 15b (29 mg, 35\%) (Overall yield 67\%). 
<smiles>C=CC(=C)C(=C)c1ccccc1</smiles>

$\stackrel{\mathrm{NMM}: 2 \mathrm{EtAICl}}{\longrightarrow}$

$3 \mathbf{b}$

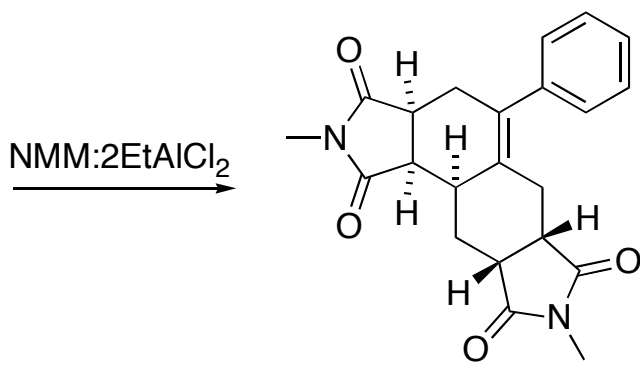

14b

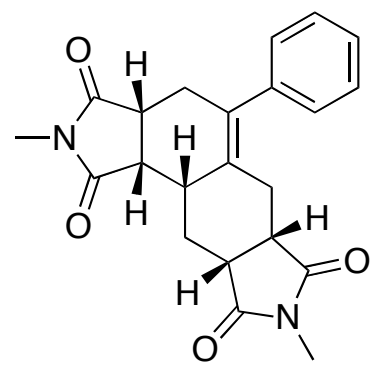

15b

Note: Commercially available $N$-methylmaleimide must be recrystallised from diethyl ether prior to use.

Ethylaluminium dichloride (3.0 $\mathrm{mL}$ of a $1 \mathrm{M}$ solution in hexanes, $3.0 \mathrm{mmol}, 19.5 \mathrm{equiv})$ was added dropwise to a stirred solution of $N$-methylmaleimide (111 mg, $1.0 \mathrm{mmol}, 6.49$ equiv) in anhydrous toluene $(0.6 \mathrm{~mL})$ at $-78{ }^{\circ} \mathrm{C}$ under nitrogen. The clear solution immediately turned deep red. After stirring for two hours at $-78^{\circ} \mathrm{C}$, a solution of 2-phenyl[3]dendralene $\mathbf{3 b}$ (24 $\mathrm{mg}, 0.154 \mathrm{mmol}, 1$ equiv) in toluene $(0.3 \mathrm{~mL})$ was added. The mixture was stirred at $-78{ }^{\circ} \mathrm{C}$ for one hour, then was allowed to warm to room temperature and stirred for 15 minutes (during which the red colour faded) and cooled again to $-78{ }^{\circ} \mathrm{C}$. Water $(10 \mathrm{~mL})$ was added and the resulting mixture was allowed to warm to room temperature. The aqueous layer was acidified with $1 \mathrm{M} \mathrm{HCl}$ (aq.) and extracted with $\mathrm{CH}_{2} \mathrm{Cl}_{2}(10 \mathrm{~mL}$ ). The organic phase was dried over anhydrous magnesium sulphate and concentrated under reduced pressure to give an oily solid. The solid was subjected to radial chromatography $(30-50 \%$ EtOAc/40 $60{ }^{\circ} \mathrm{C}$ petrol) to give the bis-adduct $14 \mathbf{b}(13 \mathrm{mg}, 22 \%)$ and bis-adduct $15 \mathbf{b}$ (26 mg, 45\%) (Overall yield $67 \%)$. 


\section{Stereochemical Assignments}

Stereochemical assignments of bis-adducts resulting from thermal and Lewis acid promoted Diels-Alder reactions

\section{Bis-adducts 14a-h}

The stereochemistry of each of the major bis-adducts 14b, 14c, 14e, 14f, 14g and 14h was assigned by comparison of ${ }^{1} \mathrm{H}$ NMR spectra with those of $\mathbf{1 4 a}$ and $\mathbf{1 4 d}$. Single crystal x-ray analyses of $\mathbf{1 4 a}$ and 14d secured stereochemical assignments of these derivatives.

Similarities between ${ }^{1} \mathrm{H}$ NMR spectra of major bis-adducts $\mathbf{1 4 a - h}$ are highlighted in Figure 1. 


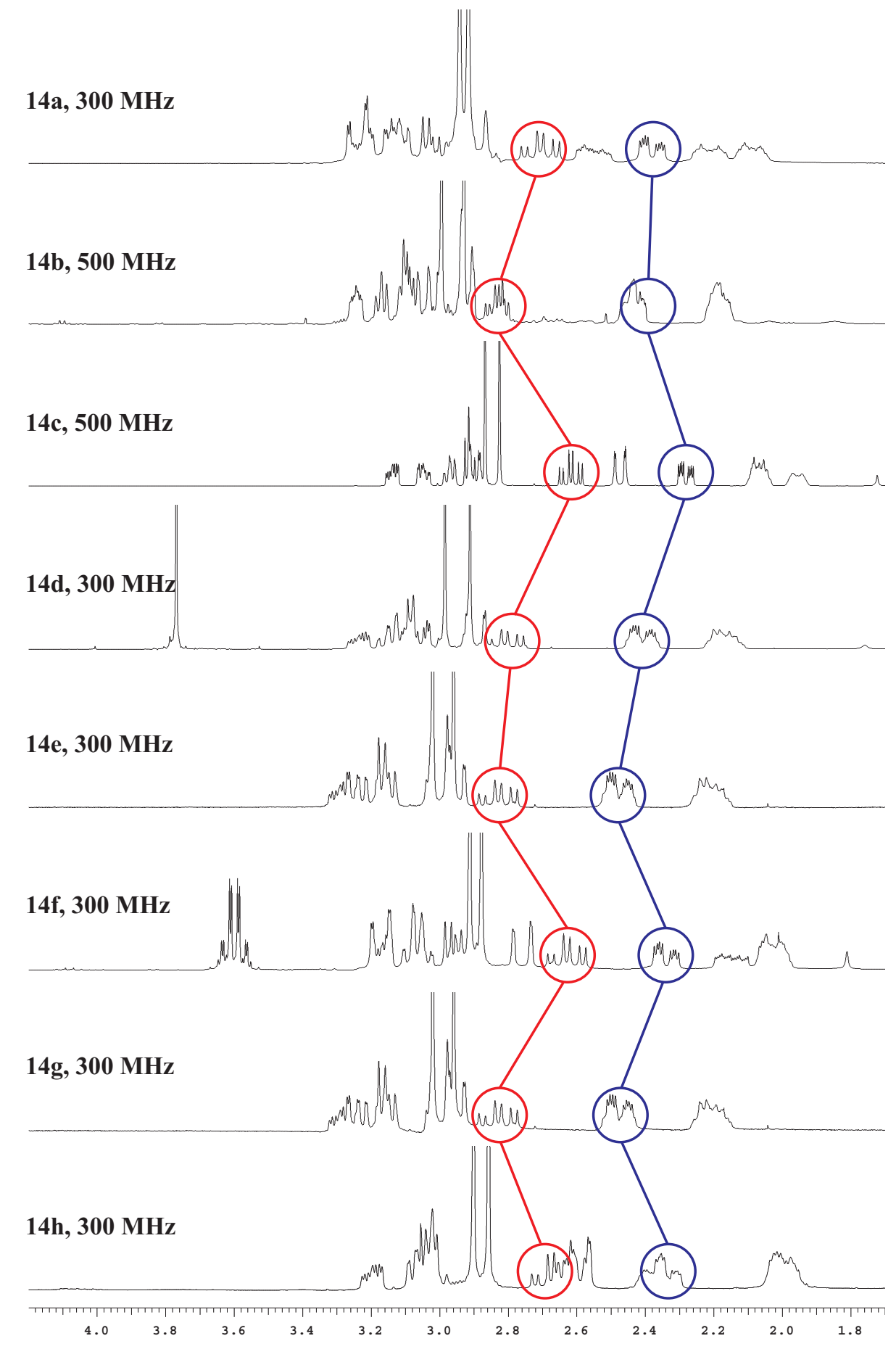

Figure 1. ${ }^{1} \mathrm{H}$ NMR spectra of major bis-adducts 14a-h 


\section{Bis-adducts 15 a-h}

The stereochemistry of each of the minor bis-adducts $\mathbf{1 5 a - f}$ and $\mathbf{h}$ was assigned by comparison of ${ }^{1} \mathrm{H}$ NMR spectra with those of $\mathbf{1 5 g}$; the stereochemistry of $\mathbf{1 5 g}$ was secured through 2D NMR experiments. The NOESY spectrum and pertinent nOe's are depicted in Figure 2.

Similarities between ${ }^{1} \mathrm{H}$ NMR spectra of major bis-adducts $\mathbf{1 5 a - h}$ are highlighted in Figure 3. 


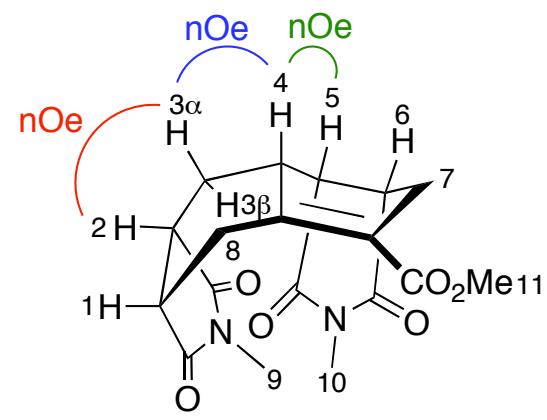

$15 \mathrm{~g}$

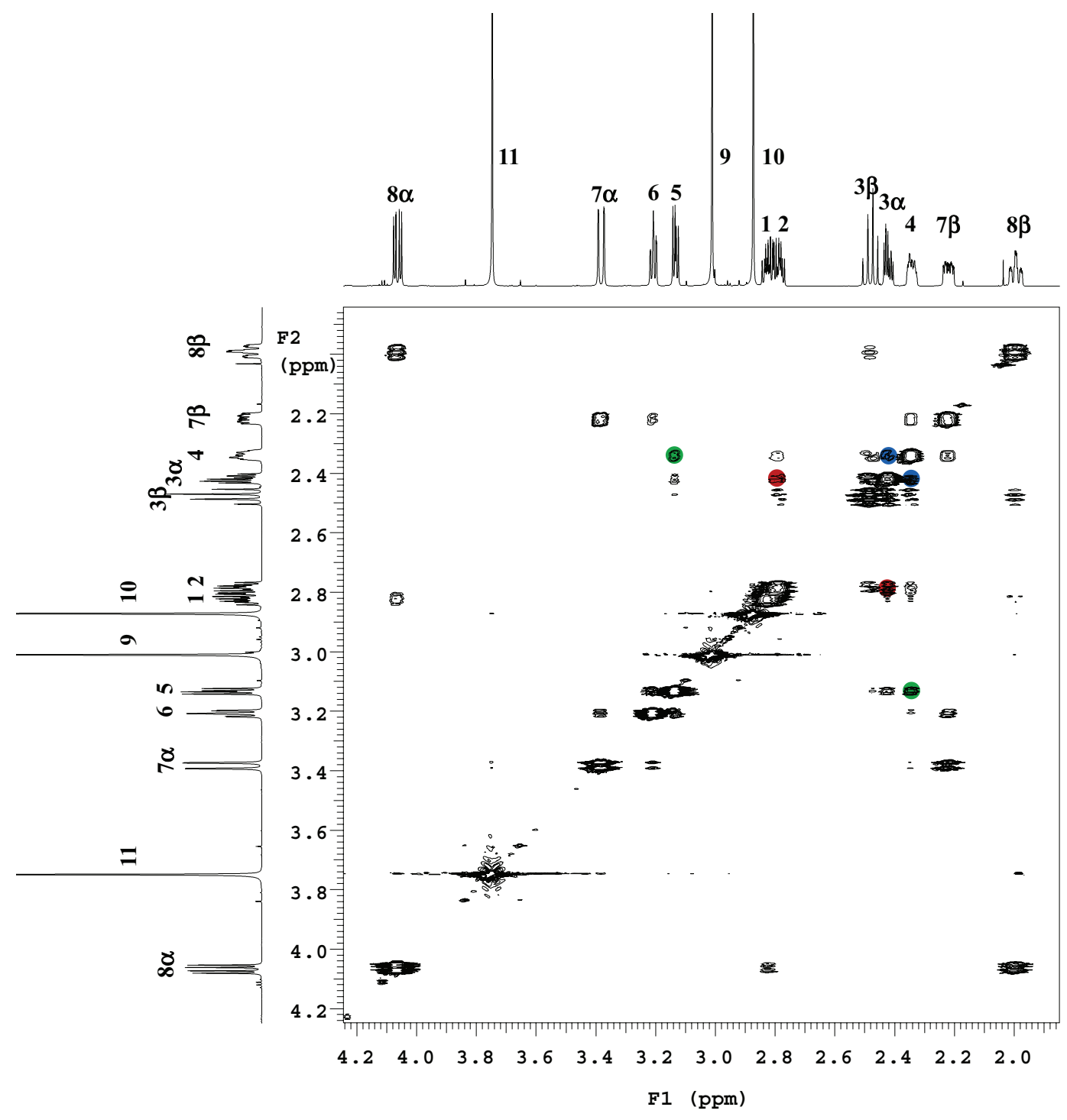

Figure 2. NOESY spectrum of bis-adduct $15 \mathrm{~g}$ 


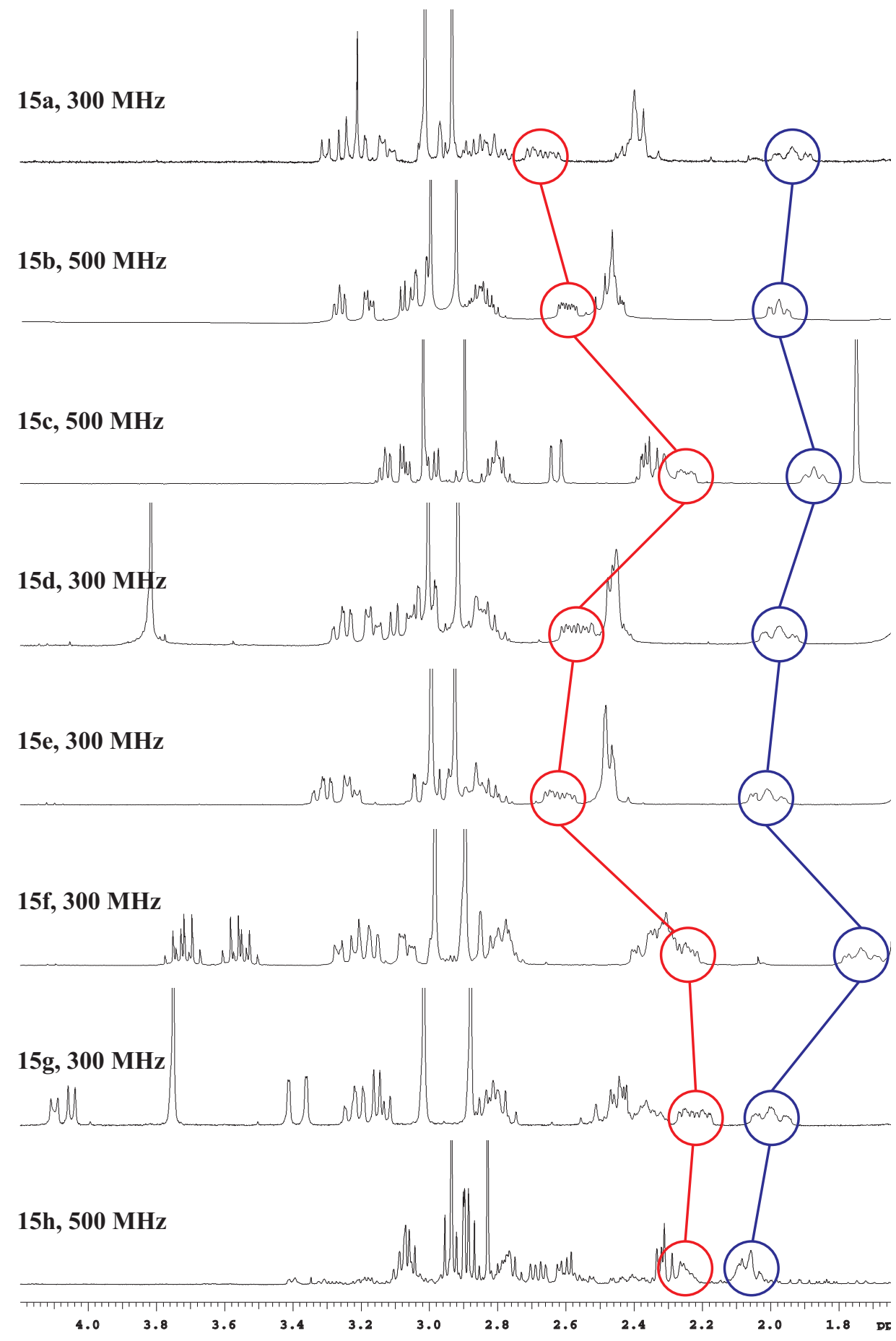

Figure 3. ${ }^{1} \mathrm{H}$ NMR spectra of minor bis-adducts 15a-h 
Stereochemical assignments of bis-adducts resulting from a high pressure Diels-Alder reaction

\section{Bis-adduct 17d}

The stereochemistry of the major bis-adduct 17d was secured through 2D NMR experiments. The NOESY spectrum and pertinent nOe's are depicted in Figure 4. 


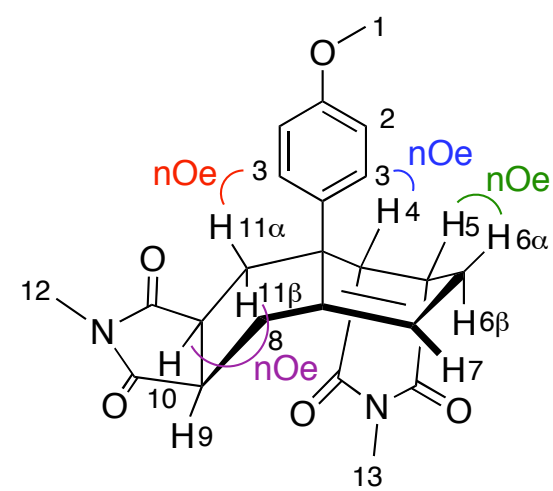

17d

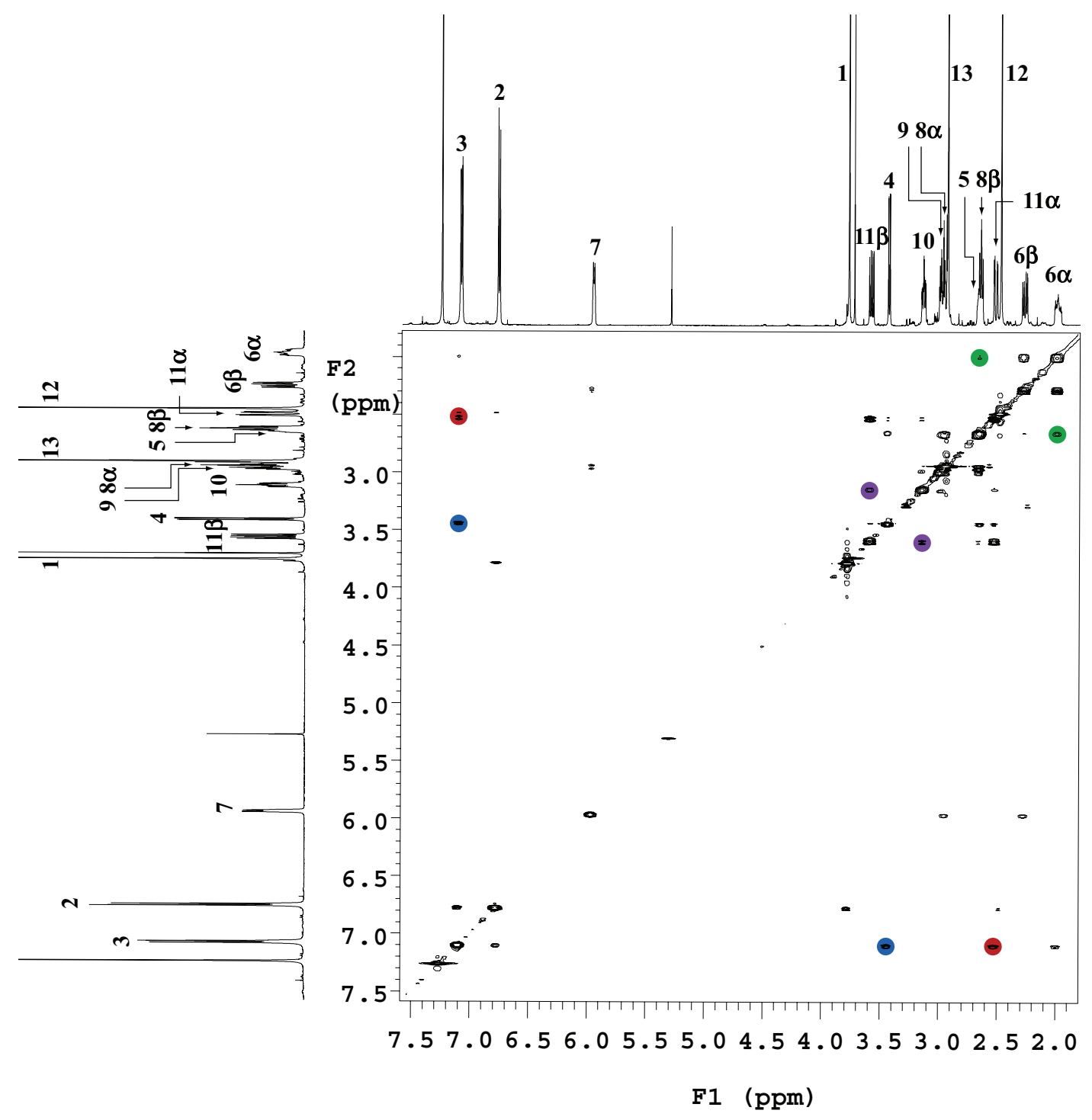

Figure 4. NOESY spectrum of bis-adduct 17d 


\section{Bis-adduct 18d}

The stereochemistry of the minor bis-adduct 18d was secured through 2D NMR experiments. The NOESY spectrum and pertinent nOe's are depicted in Figure 5. 


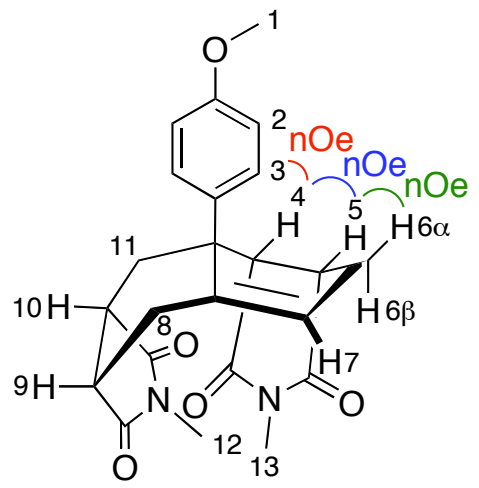

18d

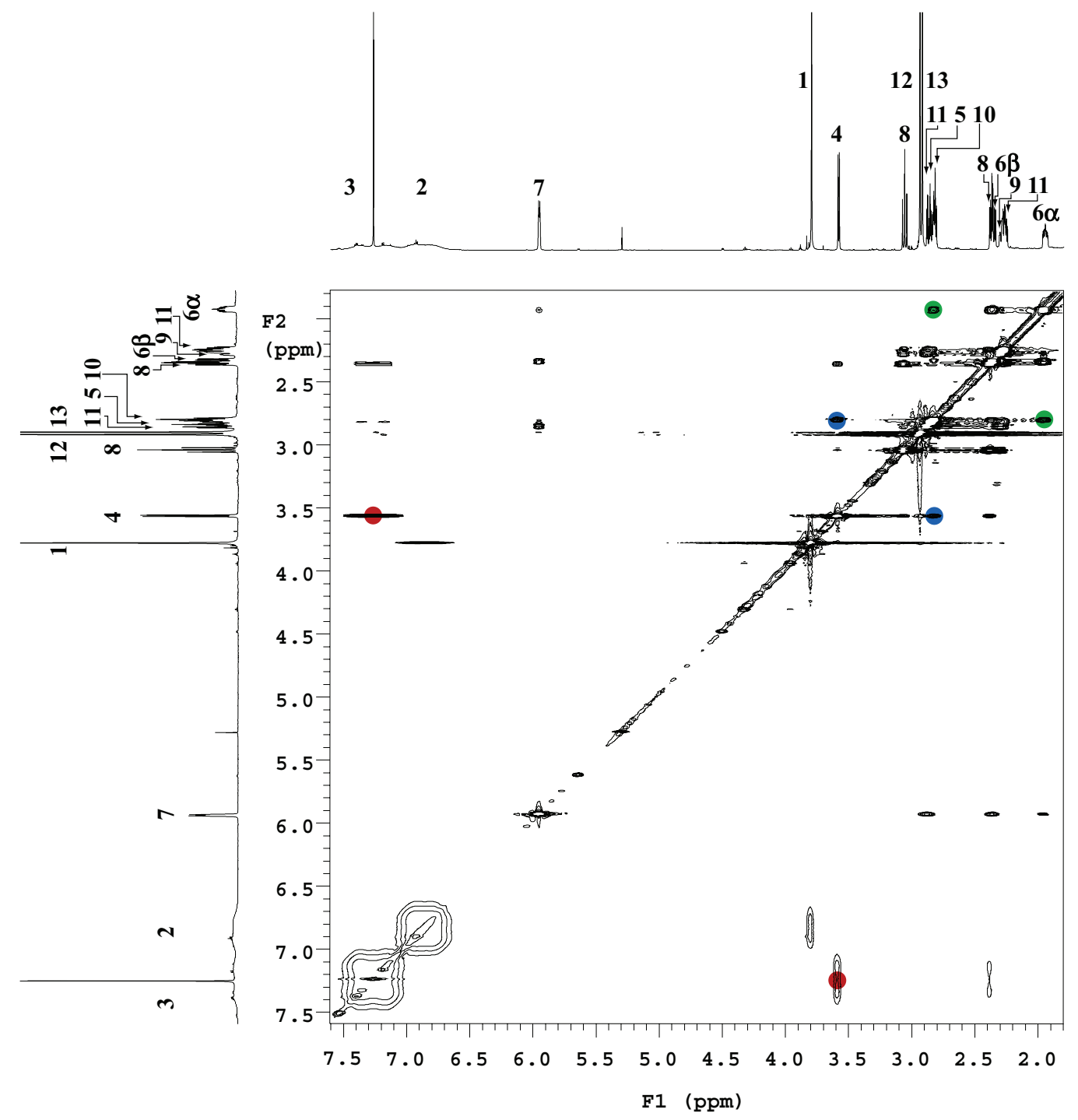

Figure 5. NOESY spectrum of bis-adduct 18d 


\section{References}

(1) Chloroprene, which is not commercially available in Australia, was prepared following the patented procedure:

Tassara, J. P.; Baudoin, M. Eur. Pat. Appl. 94-202577, 1994; Chem. Abstr. 1994, 122, 290321.

(2) Fielder, S.; Rowan, D. D.; Sherburn, M. S. Angew Chem., Int. Ed. 2000, 39, 4331 - 4333.

(3) Nunomoto, S.; Yamashita, Y. J. Org. Chem. 1979, 44, 4788 - 4791.

(4) Love, B.E.; Jones, E.G. J. Org. Chem. 1999, 64, 3755 - 3756.

(5) Lawson, K.R.; Upton, R.M. Eur. Pat. Appl. EP0206313, 1986.

(6) Carothers, W.H.; Berchet, G.J. J. Am. Chem. Soc. 1933, 55, 2807.

(7) Pornet, J.; Randrianoeline, B.; Maginia, C. L. J. Organometal. Chem. 1979, 174, 1 - 13.

(8) Yu, W.; Jin, Z. J. Am. Chem. Soc. 2000, 122, $9840-9841$.

(9) Kanemasa, S.; Sakoh, H.; Wada, E.; Tsuge, O. Bull. Chem. Soc. Jpn. 1985, 58, 3312 - 3319. 


\section{Computational Details}

All calculations were carried out using the B3LYP/6-31G(d) theoretical model and Gaussian 03:

Frisch, M. J.; Trucks, G. W.; Schlegel, H. B.; Scuseria, G. E.; Robb, M. A.; Cheeseman, J. R.; Montgomery, J. A., Jr.; Vreven, T.; Kudin, K. N.; Burant, J. C.; Millam, J. M.; Iyengar, S. S.; Tomasi, J.; Barone, V.; Mennucci, B.; Cossi, M.; Scalmani, G.; Rega, N.; Petersson, G. A.; Nakatsuji, H.; Hada, M.; Ehara, M.; Toyota, K.; Fukuda, R.; Hasegawa, J.; Ishida, M.; Nakajima, T.; Honda, Y.; Kitao, O.; Nakai, H.; Klene, M.; Li, X.; Knox, J. E.; Hratchian, H. P.; Cross, J. B.; Adamo, C.; Jaramillo, J.; Gomperts, R.; Stratmann, R. E.; Yazyev, O.; Austin, A. J.; Cammi, R.; Pomelli, C.; Ochterski, J. W.; Ayala, P. Y.; Morokuma, K.; Voth, G. A.; Salvador, P.; Dannenberg, J. J.; Zakrzewski, V. G.; Dapprich, S.; Daniels, A. D.; Strain, M. C.; Farkas, O.; Malick, D. K.; Rabuck, A. D.; Raghavachari, K.; Foresman, J. B.; Ortiz, J. V.; Cui, Q.; Baboul, A. G.; Clifford, S.; Cioslowski, J.; Stefanov, B. B.; Liu, G.; Liashenko, A.; Piskorz, P.; Komaromi, I.; Martin, R. L.; Fox, D. J.; Keith, T.; Al-Laham, M. A.; Peng, C. Y.; Nanayakkara, A.; Challacombe, M.; Gill, P. M. W.; Johnson, B.; Chen, W.; Wong, M. W.; Gonzalez, C.; Pople, J. A. Gaussian 2003, Revision D.01; Gaussian, Inc.: Pittsburgh, PA, 2003.

Eight TSs were located, four associated with the reactive diene bearing the 2-chlorovinyl substituent, denoted by A, A1, A2, and A3, and four TSs associated with the reactive diene bearing the 2-chloro-3vinyl substituents, denoted by $\mathbf{B}, \mathbf{B 1}, \mathbf{B 2}$, and $\mathbf{B 3}$. TSs $\mathbf{A}$ and $\mathbf{B}$ are those depicted in Figure 2 in the paper.

The Cartesian coordinates, energies, enthalpies and free energies of the eight TSs are listed below, in decreasing order of free energy (most negative first).

N_2-ChloroVinyl_Butdien_NMe_Maleimid_3: TS A MPR_ALTIX_APAC RB3LYP/6-31G(d)

SCF Energy $($ no zpe $)=-1091.7043893 \mathrm{au}$; Energy+zpe $(0 \mathrm{~K})=-1091.4954323 \mathrm{au}$

Enthalpy $(298 \mathrm{~K})=-1091.4799123 \mathrm{au}$; Gibbs free energy $(298 \mathrm{~K})=-1091.5376253 \mathrm{au}$

$\begin{array}{llll}\mathrm{C} & 0.281364 & -2.541330 & 0.225932 \\ \mathrm{C} & -0.715673 & -1.667843 & -0.145269 \\ \mathrm{C} & -1.185882 & -0.626474 & 0.697919 \\ \mathrm{C} & -0.615648 & -0.454204 & 1.954675 \\ \mathrm{H} & 0.440812 & -2.817541 & 1.262001 \\ \mathrm{H} & 0.708973 & -3.217074 & -0.508222 \\ \mathrm{H} & -1.023030 & -1.648976 & -1.186966 \\ \mathrm{H} & -0.230849 & -1.312907 & 2.489940 \\ \mathrm{C} & 1.563331 & -0.067330 & 1.516913 \\ \mathrm{C} & 2.045039 & -1.062696 & 0.673766 \\ \mathrm{C} & 1.368656 & 1.169322 & 0.697582 \\ \mathrm{C} & 2.139265 & -0.503423 & -0.705847 \\ \mathrm{O} & 2.571533 & -1.019327 & -1.717988 \\ \mathrm{O} & 1.049465 & 2.287599 & 1.062409 \\ \mathrm{H} & 1.758916 & 0.015295 & 2.578437\end{array}$




$\begin{array}{llll}\mathrm{H} & 2.647216 & -1.915089 & 0.958586 \\ \mathrm{C} & -2.114130 & 0.400308 & 0.158790 \\ \mathrm{C} & -2.115392 & 1.706770 & 0.436035 \\ \mathrm{H} & -2.859140 & 2.372672 & 0.013891 \\ \mathrm{H} & -1.345144 & 2.139236 & 1.066123 \\ \mathrm{~N} & 1.626052 & 0.803918 & -0.622842 \\ \mathrm{C} & 1.511075 & 1.711579 & -1.749201 \\ \mathrm{H} & 2.105124 & 2.612748 & -1.572125 \\ \mathrm{H} & 1.882749 & 1.186876 & -2.630694 \\ \mathrm{H} & 0.466869 & 2.002363 & -1.899160 \\ \mathrm{H} & -0.909736 & 0.382300 & 2.579501 \\ \mathrm{Cl} & -3.350891 & -0.233034 & -0.947915\end{array}$

\#\#\#\#\#\#\#\#\#\#\#\#\#\#\#\#\#\#\#\#\#\#\#

N_2-ChloroVinyl_ButdieneNMe_Maleimid_2: TS A1 MPR_ALTIX_APAC RB3LYP/6-31G(d) SCF Energy $($ no zpe $)=-1091.7034545 \mathrm{au}$; Energy+zpe $(0 \mathrm{~K})=-1091.4946245 \mathrm{au}$

Enthalpy $(298 \mathrm{~K})=-1091.4790005 \mathrm{au}$; Gibbs free energy $(298 \mathrm{~K})=-1091.5372415 \mathrm{au}$

$\begin{array}{llll}\mathrm{C} & -0.591950 & 2.229026 & -1.328565 \\ \mathrm{C} & 0.501994 & 1.401930 & -1.243798 \\ \mathrm{C} & 1.192195 & 1.141346 & -0.028815 \\ \mathrm{C} & 0.728718 & 1.705921 & 1.157943 \\ \mathrm{H} & -0.752202 & 3.044461 & -0.633059 \\ \mathrm{H} & -1.158172 & 2.284573 & -2.253170 \\ \mathrm{H} & 0.745482 & 0.785280 & -2.103201 \\ \mathrm{H} & 0.271717 & 2.688287 & 1.148118 \\ \mathrm{C} & -1.358382 & 0.917582 & 1.365482 \\ \mathrm{C} & -2.069849 & 1.139605 & 0.195413 \\ \mathrm{C} & -1.002358 & -0.538868 & 1.415657 \\ \mathrm{C} & -2.160157 & -0.147653 & -0.552504 \\ \mathrm{O} & -2.756474 & -0.395384 & -1.582846 \\ \mathrm{O} & -0.469585 & -1.162647 & 2.312940 \\ \mathrm{H} & -1.461021 & 1.477177 & 2.286566 \\ \mathrm{H} & -2.778062 & 1.934461 & 0.004217 \\ \mathrm{C} & 2.279402 & 0.139651 & 0.038242 \\ \mathrm{C} & 3.269861 & 0.137006 & 0.936806 \\ \mathrm{H} & 4.025990 & -0.638722 & 0.953020 \\ \mathrm{H} & 3.343951 & 0.930209 & 1.672791 \\ \mathrm{~N} & -1.419641 & -1.078164 & 0.196277 \\ \mathrm{C} & -1.266556 & -2.476488 & -0.164118 \\ \mathrm{H} & -1.575176 & -3.109449 & 0.672207 \\ \mathrm{H} & -1.900744 & -2.662134 & -1.032563 \\ \mathrm{H} & -0.225059 & -2.699228 & -0.413192 \\ \mathrm{H} & 1.145937 & 1.394411 & 2.109575 \\ \mathrm{Cl} & 2.289753 & -1.136017 & -1.198616\end{array}$

\#\#\#\#\#\#\#\#\#\#\#\#\#\#\#\#\#\#\#\#\#\#\#\# 
N_2-ChloroVinyl_Butdien_NMe_Maleimid_1: TS A2 MPR_ALTIX_APAC RB3LYP/6-31G(d)

SCF Energy (no zpe) $=-1091.7019071 \mathrm{au}$; Energy+zpe $(0 \mathrm{~K})=-1091.4931041 \mathrm{au}$

Enthalpy $(298 \mathrm{~K})=-1091.4774531 \mathrm{au}$; Gibbs free energy $(298 \mathrm{~K})=-1091.5361791 \mathrm{au}$

$\begin{array}{llll}\mathrm{C} & 0.936253 & 2.549287 & -0.199076 \\ \mathrm{C} & -0.156663 & 1.977342 & 0.411542 \\ \mathrm{C} & -1.093562 & 1.155189 & -0.274368 \\ \mathrm{C} & -0.872202 & 0.848053 & -1.612898 \\ \mathrm{H} & 0.924606 & 2.823128 & -1.248113 \\ \mathrm{H} & 1.694821 & 3.039017 & 0.403502 \\ \mathrm{H} & -0.186259 & 1.974893 & 1.497402 \\ \mathrm{H} & -0.378620 & 1.570737 & -2.249455 \\ \mathrm{C} & 1.162198 & -0.144468 & -1.636144 \\ \mathrm{C} & 2.057758 & 0.664810 & -0.945770 \\ \mathrm{C} & 0.844485 & -1.319329 & -0.764348 \\ \mathrm{C} & 2.289384 & 0.064969 & 0.403107 \\ \mathrm{O} & 3.047673 & 0.429023 & 1.282014 \\ \mathrm{O} & 0.223735 & -2.327763 & -1.033380 \\ \mathrm{H} & 1.096303 & -0.243557 & -2.712027 \\ \mathrm{H} & 2.796625 & 1.324942 & -1.380586 \\ \mathrm{C} & -2.179147 & 0.524184 & 0.513010 \\ \mathrm{C} & -2.696903 & 1.021204 & 1.642234 \\ \mathrm{H} & -3.504309 & 0.523091 & 2.165258 \\ \mathrm{H} & -2.333974 & 1.957318 & 2.053014 \\ \mathrm{~N} & 1.446241 & -1.053061 & 0.473321 \\ \mathrm{C} & 1.372382 & -1.949362 & 1.612428 \\ \mathrm{H} & 1.572179 & -2.975379 & 1.291841 \\ \mathrm{H} & 2.123889 & -1.625879 & 2.334538 \\ \mathrm{H} & 0.379131 & -1.913153 & 2.070769 \\ \mathrm{H} & -1.504674 & 0.131576 & -2.124296 \\ \mathrm{Cl} & -2.879916 & -0.963333 & -0.135439\end{array}$

\#\#\#\#\#\#\#\#\#\#\#\#\#\#\#\#\#\#\#\#\#\#\#\#

N_2-ChloroVinyl_Butdien_NMe_Maleimid_4: TS A3 MPR_ALTIX_APAC RB3LYP/6-31G(d) SCF Energy $($ no zpe $)=-1091.7022195 \mathrm{au}$; Energy+zpe $(0 \mathrm{~K})=-1091.4933495 \mathrm{au}$ Enthalpy $(298 \mathrm{~K})=-1091.4777885 \mathrm{au}$; Gibbs free energy $(298 \mathrm{~K})=-1091.5358205 \mathrm{au}$
C $\quad-0.839232 \quad 2.544835 \quad-0.450616$
C $\quad 0.183107 \quad 1.765223 \quad-0.939497$
C $\quad 1.085561 \quad 1.051615 \quad-0.105208$
C $\quad 0.956927 \quad 1.150189 \quad 1.276747$
$\mathrm{H} \quad-0.776959 \quad 3.027305 \quad 0.517247$
$\mathrm{H} \quad-1.577258 \quad 2.958355-1.131181$
$\mathrm{H} \quad 0.200966 \quad 1.558817 \quad-2.005872$
$\mathrm{H} \quad 0.589647 \quad 2.068191 \quad 1.718715$
$\begin{array}{llll}\text { C } & -1.085574 & 0.333946 & 1.669458\end{array}$
$\begin{array}{llll}\text { C } & -2.016593 & 0.961233 & 0.849202\end{array}$
$\begin{array}{llll}\text { C } & -0.848683 & -1.041288 & 1.121614\end{array}$
$\begin{array}{llll}\text { C } & -2.360430 & 0.035293 & -0.268671\end{array}$ 


$\begin{array}{llll}\mathrm{O} & -3.169738 & 0.179481 & -1.165298 \\ \mathrm{O} & -0.195926 & -1.951581 & 1.591966 \\ \mathrm{H} & -0.949019 & 0.519851 & 2.727314 \\ \mathrm{H} & -2.696782 & 1.754504 & 1.128828 \\ \mathrm{C} & 1.987975 & 0.051244 & -0.724856 \\ \mathrm{C} & 1.805243 & -0.572202 & -1.894693 \\ \mathrm{H} & 2.544892 & -1.253921 & -2.297220 \\ \mathrm{H} & 0.899887 & -0.417237 & -2.471281 \\ \mathrm{~N} & -1.554634 & -1.100275 & -0.084719 \\ \mathrm{C} & -1.569851 & -2.264438 & -0.948954 \\ \mathrm{H} & -1.814393 & -3.159626 & -0.370347 \\ \mathrm{H} & -2.327204 & -2.094790 & -1.716135 \\ \mathrm{H} & -0.589447 & -2.408017 & -1.415351 \\ \mathrm{H} & 1.568773 & 0.534848 & 1.926504 \\ \mathrm{Cl} & 3.467291 & -0.311968 & 0.180077\end{array}$

\section{\#\#\#\#\#\#\#\#\#\#\#\#\#\#\#\#\#\#\#\#\#\#\#\#}

N_2-Cl_3_Vinyl_Butdien_NMe_Maleimid_2: TS B MPR_ALTIX_APAC RB3LYP/6-31G(d)

SCF Energy (no zpe) $=-1091.7012515 \mathrm{au} ;$ Energy+zpe $(0 \overline{\mathrm{K}})=-1091.4928025 \mathrm{au}$

Enthalpy $(298 \mathrm{~K})=-1091.4769425 \mathrm{au}$; Gibbs free energy $(298 \mathrm{~K})=-1091.5357535 \mathrm{au}$

$\begin{array}{llll}\mathrm{C} & -0.412158 & -1.703615 & 1.540251 \\ \mathrm{C} & -1.168609 & -1.149512 & 0.536232 \\ \mathrm{C} & -1.575127 & 0.221152 & 0.507063 \\ \mathrm{C} & -1.187740 & 1.027839 & 1.574607 \\ \mathrm{H} & -0.465644 & -1.305567 & 2.544624 \\ \mathrm{H} & -0.027321 & -2.712025 & 1.441783 \\ \mathrm{H} & -1.085608 & 0.625948 & 2.574836 \\ \mathrm{C} & 1.063914 & 1.002072 & 1.491708 \\ \mathrm{C} & 1.515938 & -0.309794 & 1.490469 \\ \mathrm{C} & 1.190036 & 1.534492 & 0.096080 \\ \mathrm{C} & 1.917986 & -0.666496 & 0.099222 \\ \mathrm{O} & 2.435453 & -1.684193 & -0.318324 \\ \mathrm{O} & 0.991773 & 2.663538 & -0.308253 \\ \mathrm{H} & 1.088445 & 1.683769 & 2.332316 \\ \mathrm{H} & 1.901348 & -0.866242 & 2.334346 \\ \mathrm{C} & -2.185780 & 0.816471 & -0.694070 \\ \mathrm{C} & -2.934642 & 1.926805 & -0.698497 \\ \mathrm{H} & -3.333759 & 2.325532 & -1.626016 \\ \mathrm{H} & -3.183714 & 2.469757 & 0.209287 \\ \mathrm{~N} & 1.597170 & 0.449024 & -0.689250 \\ \mathrm{C} & 1.832835 & 0.515465 & -2.119442 \\ \mathrm{H} & 2.871519 & 0.255865 & -2.343357 \\ \mathrm{H} & 1.176436 & -0.182081 & -2.648432 \\ \mathrm{H} & 1.625370 & 1.537924 & -2.439021 \\ \mathrm{H} & -1.365778 & 2.096667 & 1.518811 \\ \mathrm{H} & -2.021199 & 0.295637 & -1.632539 \\ \mathrm{Cl} & -1.418707 & -2.146611 & -0.904177\end{array}$


N_2-Cl_3_Vinyl_Butdien_NMe_Maleimid_1: TS B1 MPR_ALTIX_APAC RB3LYP/6-31G(d)

SCF Energy (no zpe) $=-1091.7018743 \mathrm{au}$; Energy+zpe $(0 \mathrm{~K})=-1091.4932883 \mathrm{au}$

Enthalpy $(298 \mathrm{~K})=-1091.4775363 \mathrm{au}$; Gibbs free energy $(298 \mathrm{~K})=-1091.5356493 \mathrm{au}$

C $\quad-1.109978 \quad-1.520230 \quad 1.444716$

$\begin{array}{llll}\text { C } & -1.588434 & -0.622018 & 0.521410\end{array}$

$\begin{array}{llll}\text { C } & -1.401546 & 0.792337 & 0.616448\end{array}$

C $\quad-0.714486 \quad 1.271594 \quad 1.727467$

$\mathrm{H} \quad-1.006518 \quad-1.231335 \quad 2.482362$

$\mathrm{H} \quad-1.145965 \quad-2.584248 \quad 1.241941$

$\begin{array}{llll}\mathrm{H} & -0.752307 & 0.746718 & 2.672658\end{array}$

C $\quad 1.337173 \quad 0.363926 \quad 1.555871$

C $\quad 1.218164 \quad-1.013391 \quad 1.425437$

$\begin{array}{llll}\text { C } & 1.667283 & 0.923573 & 0.205843\end{array}$

$\begin{array}{llll}\text { C } & 1.432380 & -1.371030 & -0.006149\end{array}$

$\begin{array}{llll}\mathrm{O} & 1.474910 & -2.468848 & -0.526022\end{array}$

$\begin{array}{llll}\mathrm{O} & 1.941398 & 2.069992 & -0.099781\end{array}$

$\begin{array}{llll}\mathrm{H} & 1.641186 & 0.894532 & 2.449366\end{array}$

$\begin{array}{llll}\mathrm{H} & 1.351763 & -1.754258 & 2.202384\end{array}$

$\begin{array}{llll}\text { C } & -1.773627 & 1.681074 & -0.501277\end{array}$

$\begin{array}{llll}\text { C } & -1.185699 & 2.842602 & -0.816983\end{array}$

$\mathrm{H} \quad-1.551244 \quad 3.432644 \quad-1.652176$

$\begin{array}{llll}\mathrm{H} & -0.314196 & 3.226031 & -0.295257\end{array}$

$\begin{array}{llll}\mathrm{N} & 1.602152 & -0.149276 & -0.682069\end{array}$

$\begin{array}{llll}\text { C } & 1.800761 & -0.027025 & -2.114212\end{array}$

$\begin{array}{llll}\mathrm{H} & 2.753488 & 0.467860 & -2.323189\end{array}$

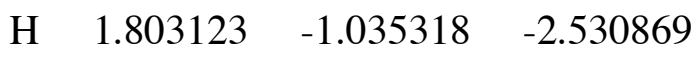

$\begin{array}{llll}\mathrm{H} & 0.992028 & 0.559427 & -2.560407\end{array}$

$\begin{array}{llll}\mathrm{H} & -0.512733 & 2.334597 & 1.806313\end{array}$

$\mathrm{H} \quad-2.602057 \quad 1.331967 \quad-1.111610$

$\begin{array}{llll}\mathrm{Cl} & -2.193767 & -1.298901 & -0.997179\end{array}$

\section{\#\#\#\#\#\#\#\#\#\#\#\#\#\#\#\#\#\#\#\#\#\#\#\#\#}

N_2-Cl_3_Vinyl_Butdien_NMe_Maleimid_4: TS B2 MPR_ALTIX_APAC RB3LYP/6-31G(d)

SCF Energy (no zpe) $=-1091.6986044 \mathrm{au}$; Energy+zpe $(0 \mathrm{~K})=-1091.4901124$ au

Enthalpy $(298 \mathrm{~K})=-1091.4742974 \mathrm{au}$; Gibbs free energy $(298 \mathrm{~K})=-1091.5327064 \mathrm{au}$
C $\quad-0.611720 \quad-1.509942 \quad 1.633436$
$\begin{array}{llll}\text { C } & -1.310371 & -0.738397 & 0.736206\end{array}$
C $\quad-1.328144 \quad 0.692720 \quad 0.781210$

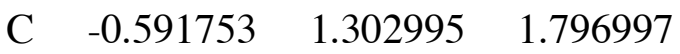
$\mathrm{H} \quad-0.434918 \quad-1.158942 \quad 2.641209$
$\mathrm{H} \quad-0.504516 \quad-2.575590 \quad 1.468535$
$\mathrm{H} \quad-0.462242 \quad 0.831281 \quad 2.762183$
C $\quad 1.518994 \quad 0.726312 \quad 1.380480$
C $\quad 1.619420 \quad-0.656318 \quad 1.325425$ 


$\begin{array}{llll}\mathrm{C} & 1.557002 & 1.244608 & -0.027293 \\ \mathrm{C} & 1.708427 & -1.066774 & -0.103795 \\ \mathrm{O} & 1.882558 & -2.172400 & -0.577595 \\ \mathrm{O} & 1.564580 & 2.398434 & -0.415275 \\ \mathrm{H} & 1.846118 & 1.356414 & 2.198006 \\ \mathrm{H} & 1.955386 & -1.318718 & 2.111951 \\ \mathrm{C} & -1.863442 & 1.585954 & -0.264161 \\ \mathrm{C} & -2.950711 & 1.424120 & -1.028723 \\ \mathrm{H} & -3.245948 & 2.205920 & -1.722639 \\ \mathrm{H} & -3.584656 & 0.546476 & -0.985409 \\ \mathrm{~N} & 1.569305 & 0.118653 & -0.849561 \\ \mathrm{C} & 1.552017 & 0.171616 & -2.299916 \\ \mathrm{H} & 2.256893 & 0.930196 & -2.649431 \\ \mathrm{H} & 1.840574 & -0.813920 & -2.668674 \\ \mathrm{H} & 0.550548 & 0.420605 & -2.665346 \\ \mathrm{H} & -0.515606 & 2.386436 & 1.804219 \\ \mathrm{H} & -1.309616 & 2.520107 & -0.351053 \\ \mathrm{Cl} & -1.926622 & -1.590028 & -0.684286\end{array}$

\#\#\#\#\#\#\#\#\#\#\#\#\#\#\#\#\#\#\#\#\#\#\#

N_2-Cl_3_Vinyl_Butdien_NMe_Maleimid_3: TS B3 MPR_ALTIX_APAC RB3LYP/6-31G(d) SCF Energy (no zpe) $=-1091.6986043 \mathrm{au}$; Energy+zpe $(0 \mathrm{~K})=-1091.4901123 \mathrm{au}$ Enthalpy $(298 \mathrm{~K})=-1091.4742983 \mathrm{au}$; Gibbs free energy $(298 \mathrm{~K})=-1091.5327053 \mathrm{au}$
$\begin{array}{llll}\text { C } & -0.611682 & -1.509857 & 1.633527\end{array}$
$\begin{array}{llll}\text { C } & -1.310324 & -0.738488 & 0.736142\end{array}$
$\begin{array}{llll}\text { C } & -1.328331 & 0.692614 & 0.781005\end{array}$
C $\quad-0.592065 \quad 1.303183 \quad 1.796706$
$\mathrm{H} \quad-0.435027 \quad-1.158718 \quad 2.641277$
$\mathrm{H} \quad-0.504321 \quad-2.575512 \quad 1.468751$

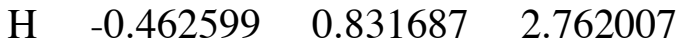
$\begin{array}{llll}\text { C } & 1.518687 & 0.726847 & 1.380452\end{array}$
$\begin{array}{llll}\text { C } & 1.619456 & -0.655781 & 1.325694\end{array}$
$\begin{array}{llll}\text { C } & 1.556831 & 1.244849 & -0.027434\end{array}$
$\begin{array}{llll}\text { C } & 1.708737 & -1.066526 & -0.103415\end{array}$
$\begin{array}{llll}\mathrm{O} & 1.883138 & -2.172217 & -0.576969\end{array}$
$\begin{array}{llll}\mathrm{O} & 1.564210 & 2.398593 & -0.415671\end{array}$
$\begin{array}{llll}\mathrm{H} & 1.845637 & 1.357170 & 2.197878\end{array}$
$\begin{array}{llll}\mathrm{H} & 1.955453 & -1.317940 & 2.112410\end{array}$
$\begin{array}{llll}\text { C } & -1.863741 & 1.585463 & -0.264647\end{array}$
$\begin{array}{llll}\text { C } & -2.951537 & 1.423427 & -1.028401\end{array}$

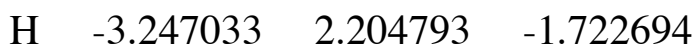
$\begin{array}{llll}\mathrm{H} & -3.585656 & 0.545950 & -0.984034\end{array}$
$\begin{array}{llll}\mathrm{N} & 1.569553 & 0.118732 & -0.849460\end{array}$
$\begin{array}{llll}\text { C } & 1.552427 & 0.171344 & -2.299826\end{array}$
$\begin{array}{llll}\mathrm{H} & 2.257657 & 0.929520 & -2.649503\end{array}$
$\mathrm{H} \quad 1.840592 \quad-0.814413 \quad-2.668307$
$\begin{array}{llll}\mathrm{H} & 0.551090 & 0.420663 & -2.665402\end{array}$ 

$\begin{array}{llll}\mathrm{H} & -0.516089 & 2.386642 & 1.803693\end{array}$
$\mathrm{H} \quad-1.309640 \quad 2.519374 \quad-0.352415$
Cl $\quad-1.926263 \quad-1.590223 \quad-0.684421$
\#\#\#\#\#\#\#\#\#\#\#\#\#\#\#\#\#\#\#\#\#\#\#\#

Powerbook:\% TSs 45 -> 


\section{Anisotropic Displacement Ellipsoid Plots for 14a and 14d}

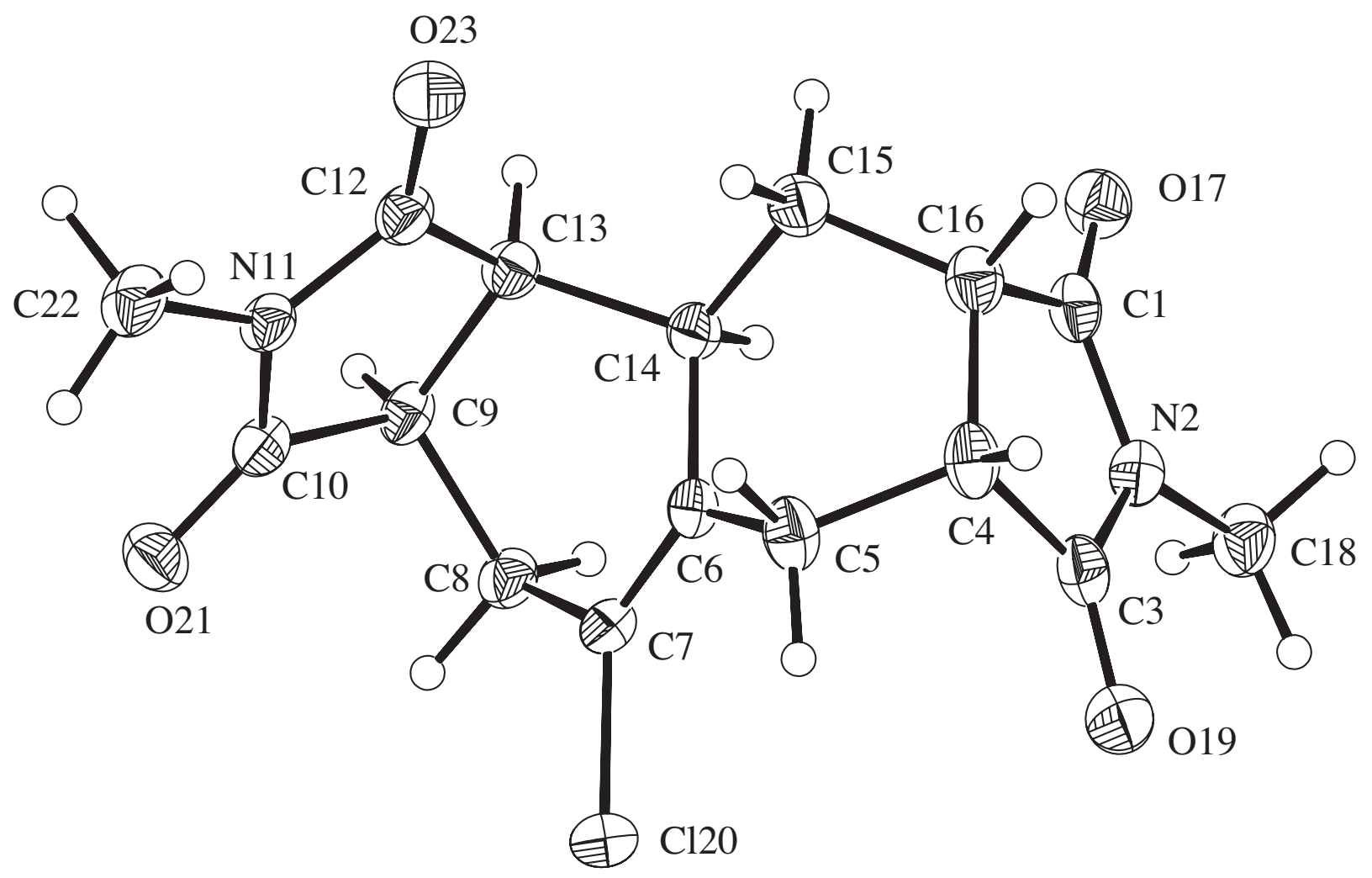

FIGURE S1. Molecular structure of compound 14a (CCDC 654085) with labeling of selected atoms. Anisotropic displacement ellipsoids show $30 \%$ probability levels. Hydrogen atoms are drawn as circles with small radii. 


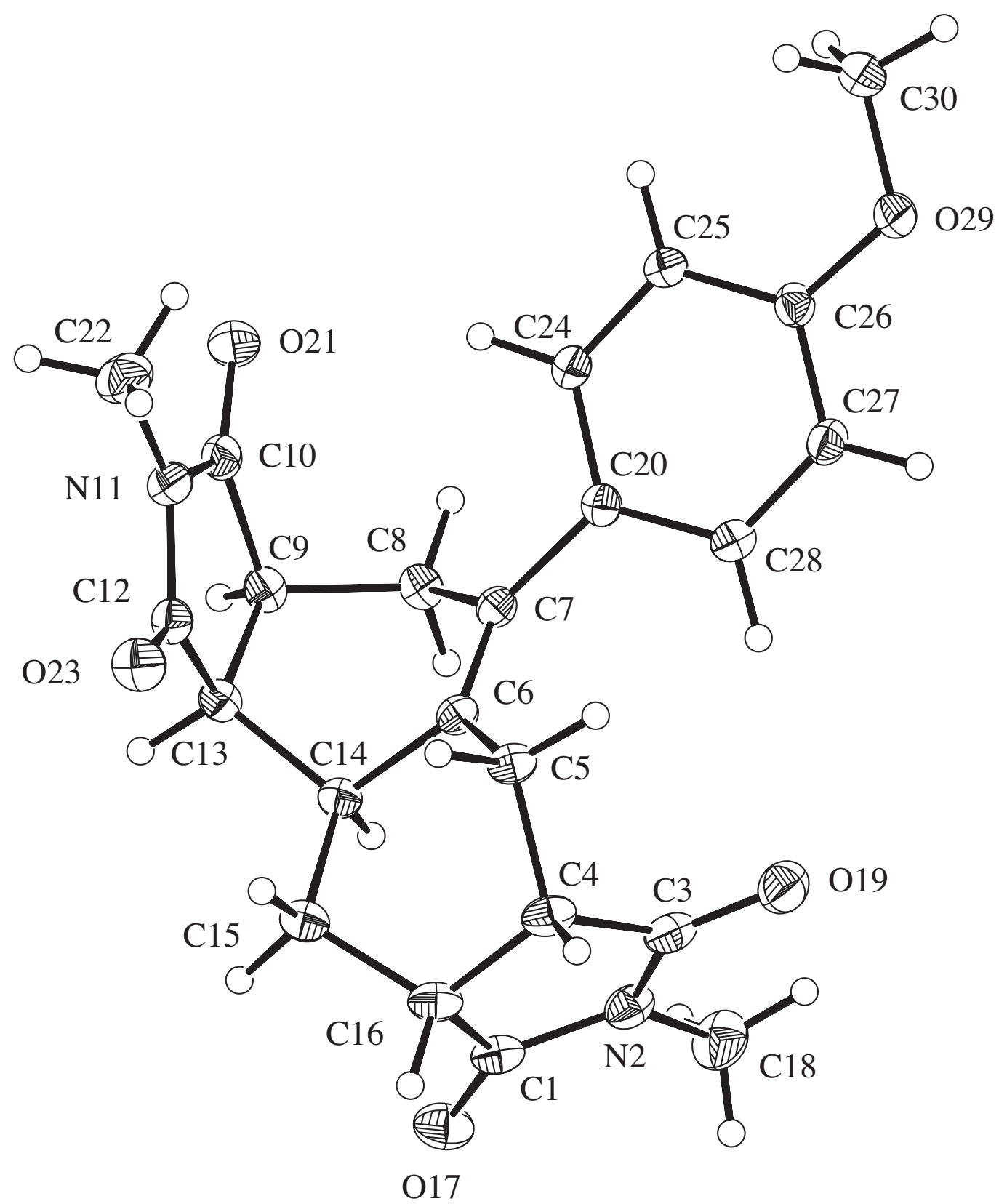

FIGURE S2. Molecular structure of compound 14d (CCDC 654086) with labeling of selected atoms. Anisotropic displacement ellipsoids show $30 \%$ probability levels. Hydrogen atoms are drawn as circles with small radii. 


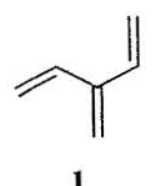

THF solution: $500 \mathrm{MHz}, \mathrm{CDCl}_{3}$

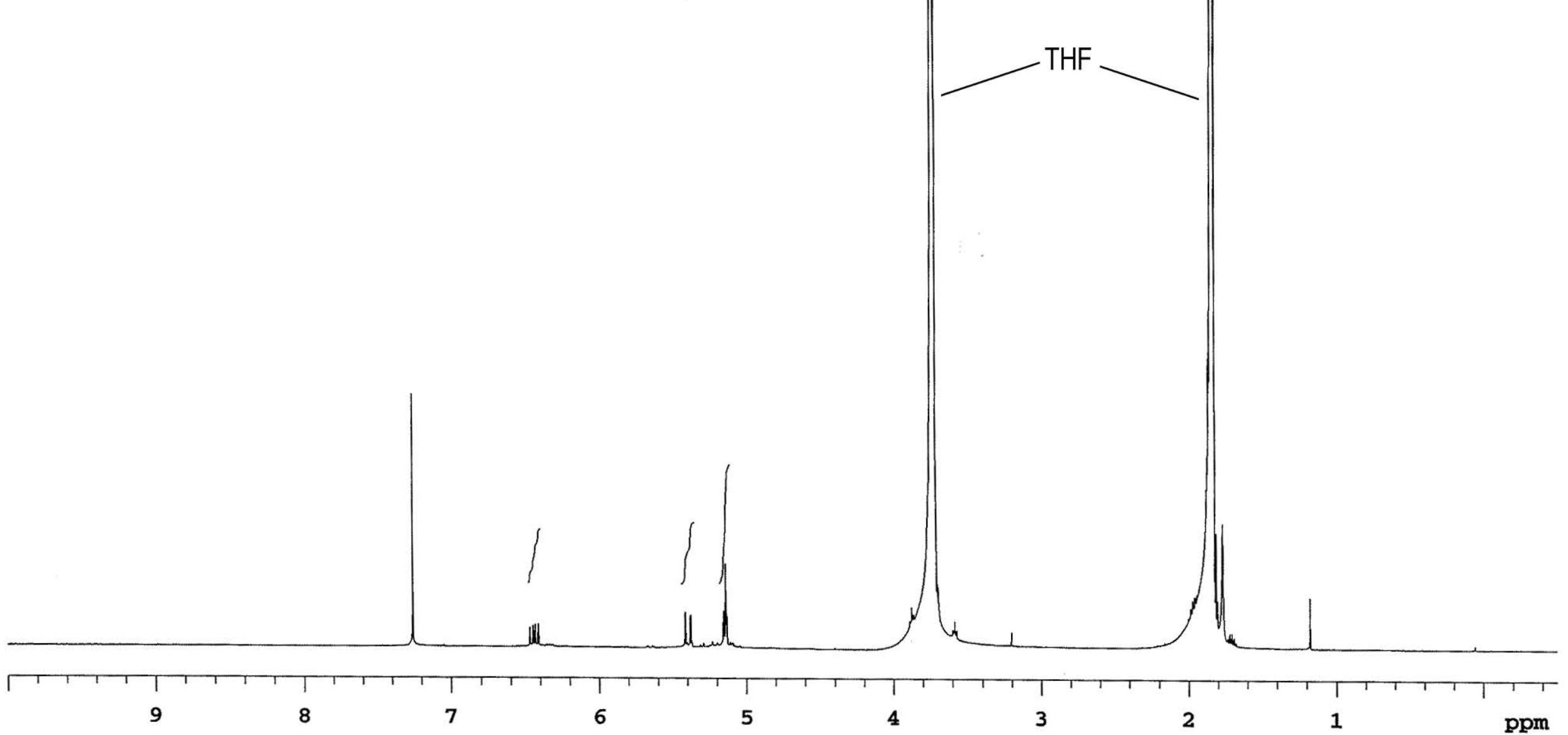




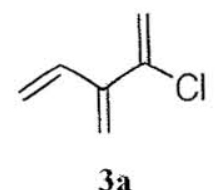

$300 \mathrm{MHz}, \mathrm{CDCl}_{3}$

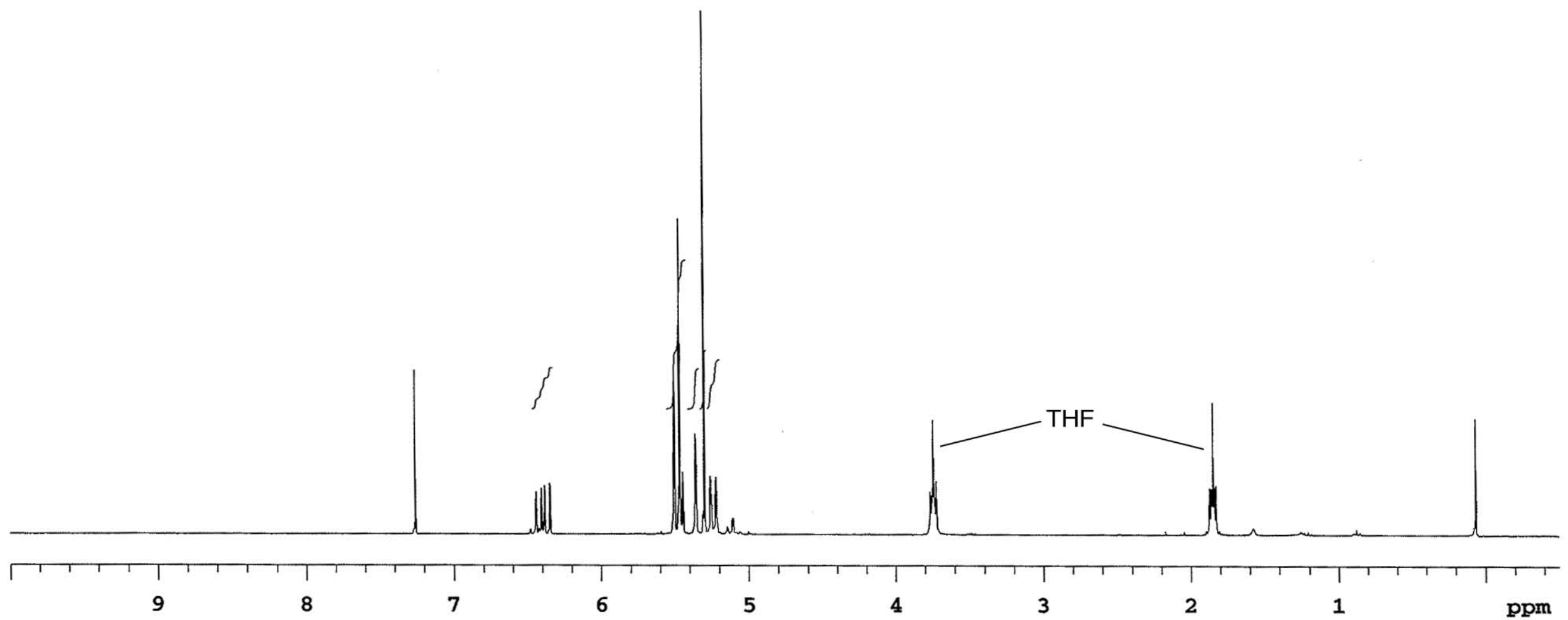




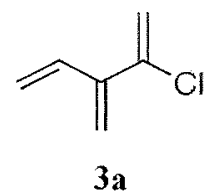

$75 \mathrm{MHz}^{\mathrm{CDCl}_{3}}$

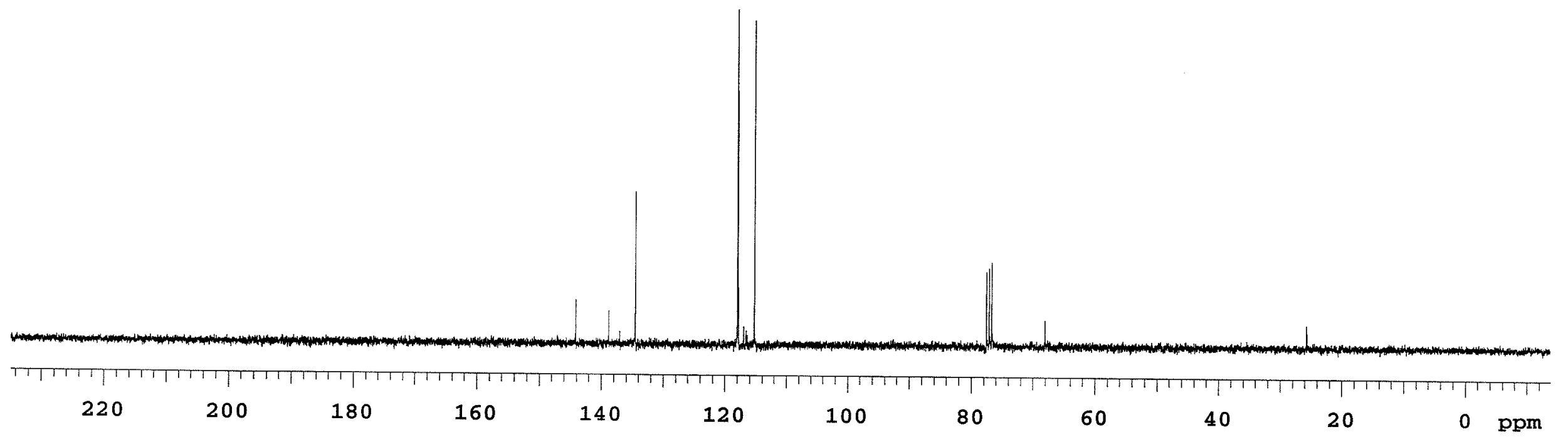




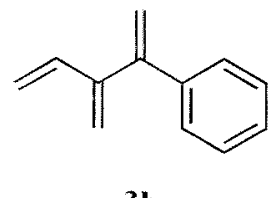

$300 \mathrm{MHz} \mathrm{CDCl}_{3}$

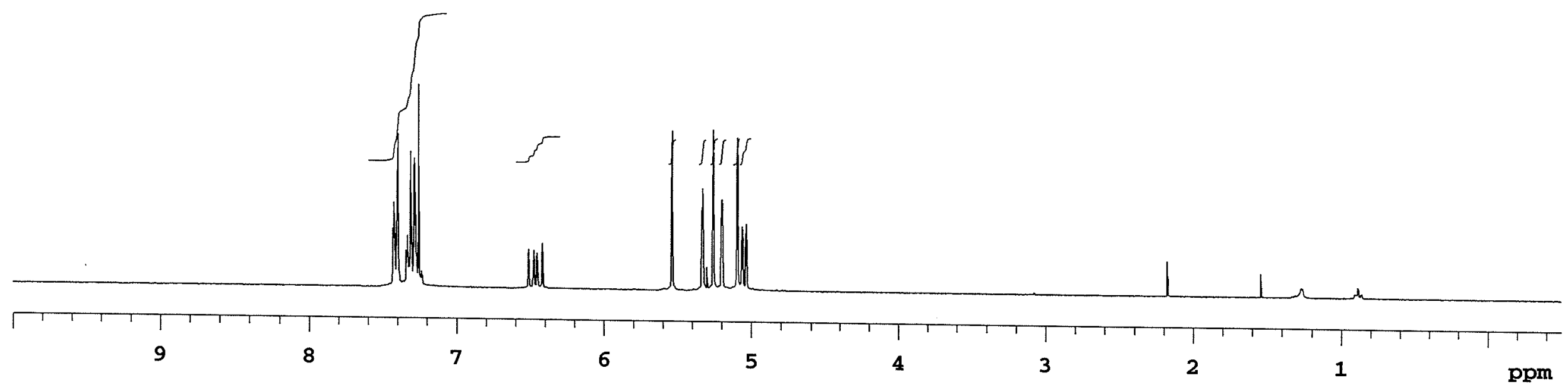




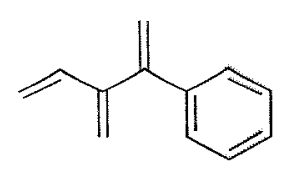

3b

$75 \mathrm{MHz}, \mathrm{CDCl}_{3}$

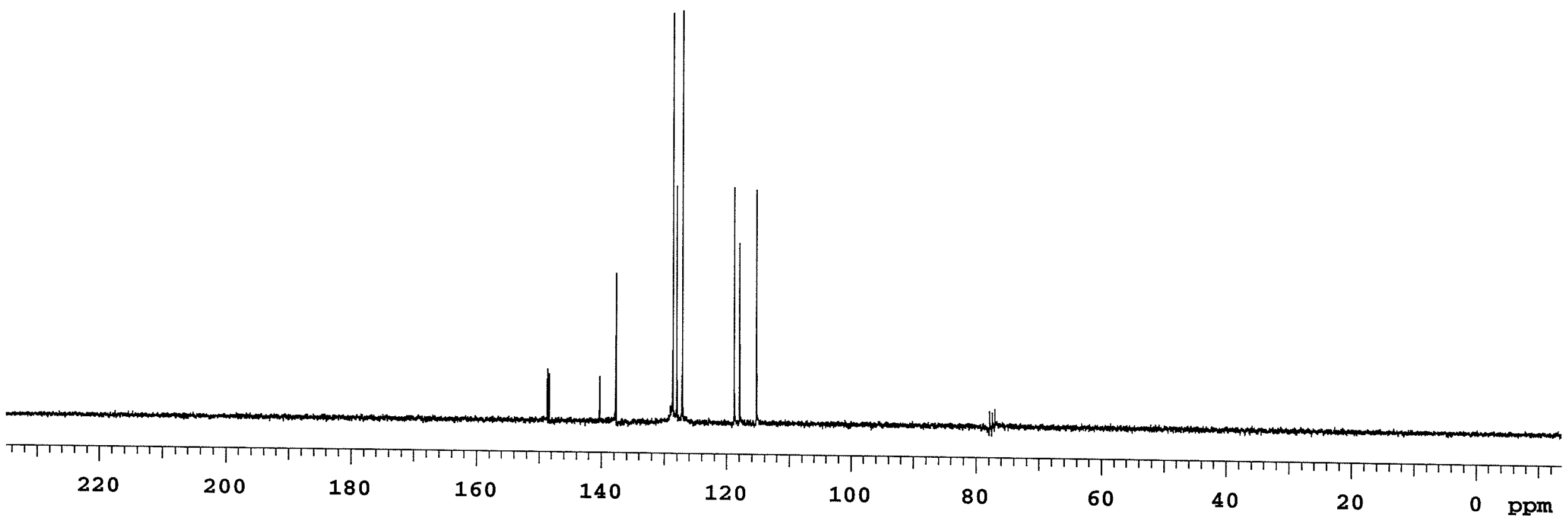




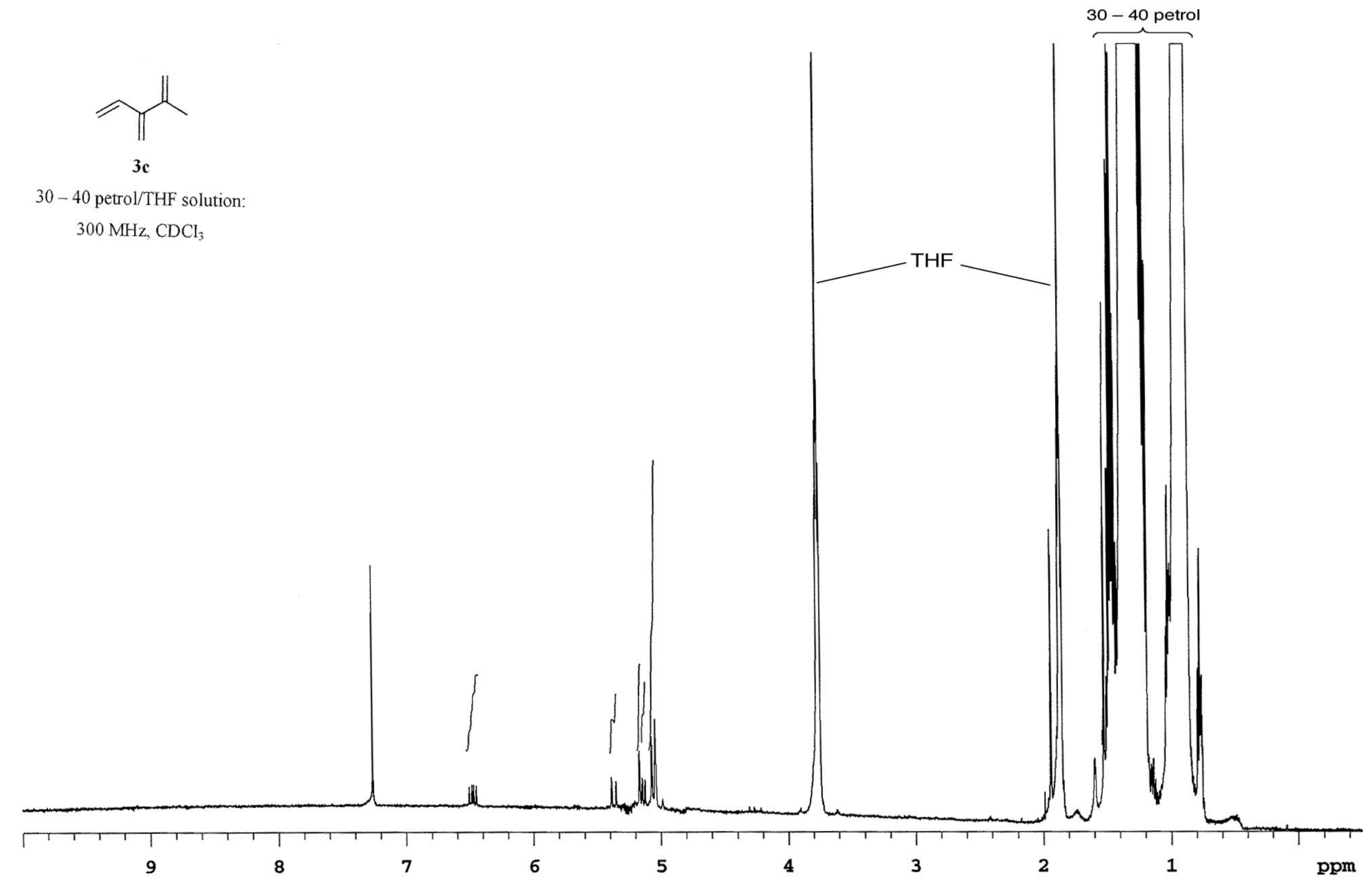




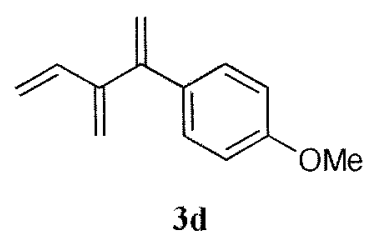

$300 \mathrm{MHz}_{3} \mathrm{CDCl}_{3}$

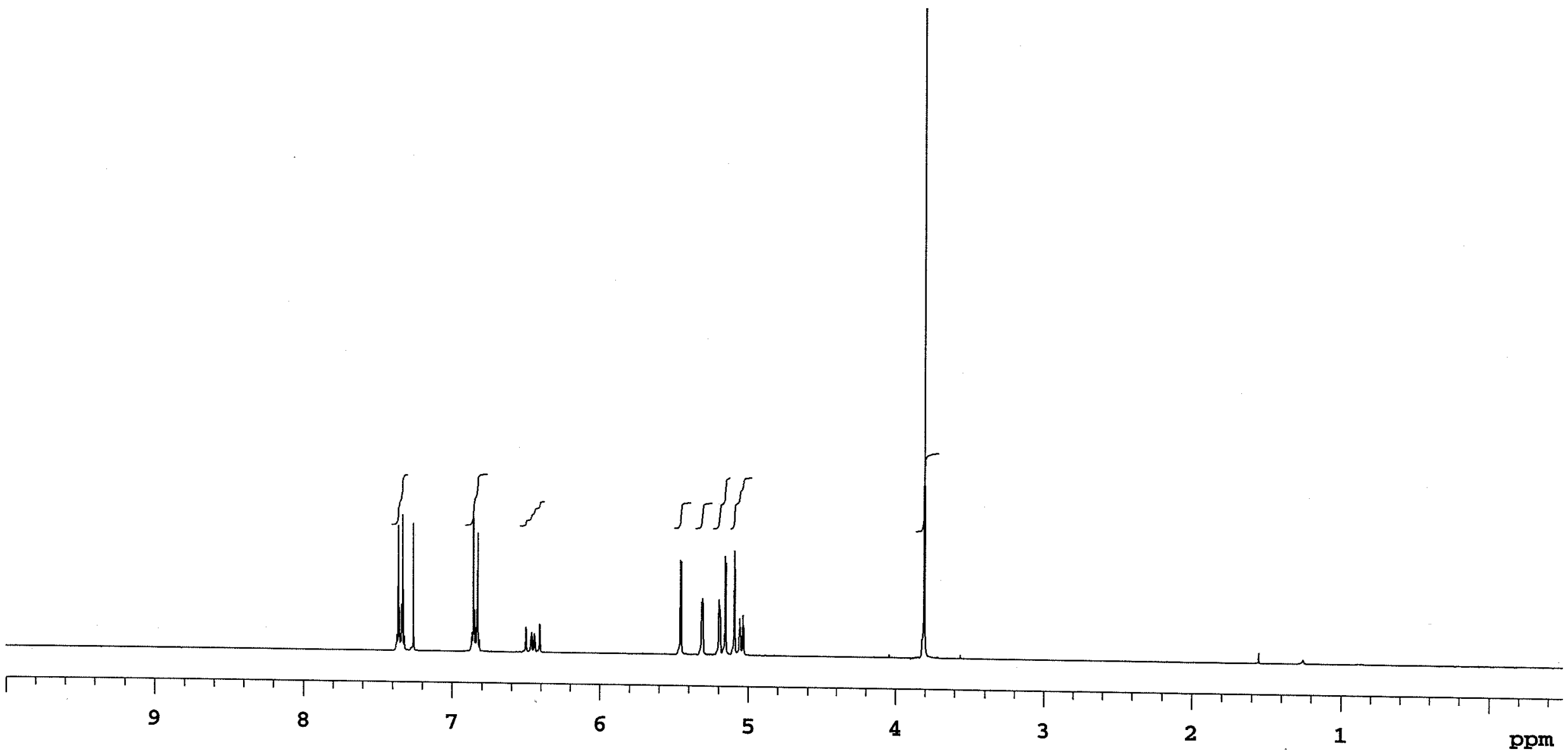


<smiles>C=CC(=C)C(=C)c1ccc(OC)cc1</smiles>

3d

$75 \mathrm{MHz}_{2} \mathrm{CDCl}_{3}$

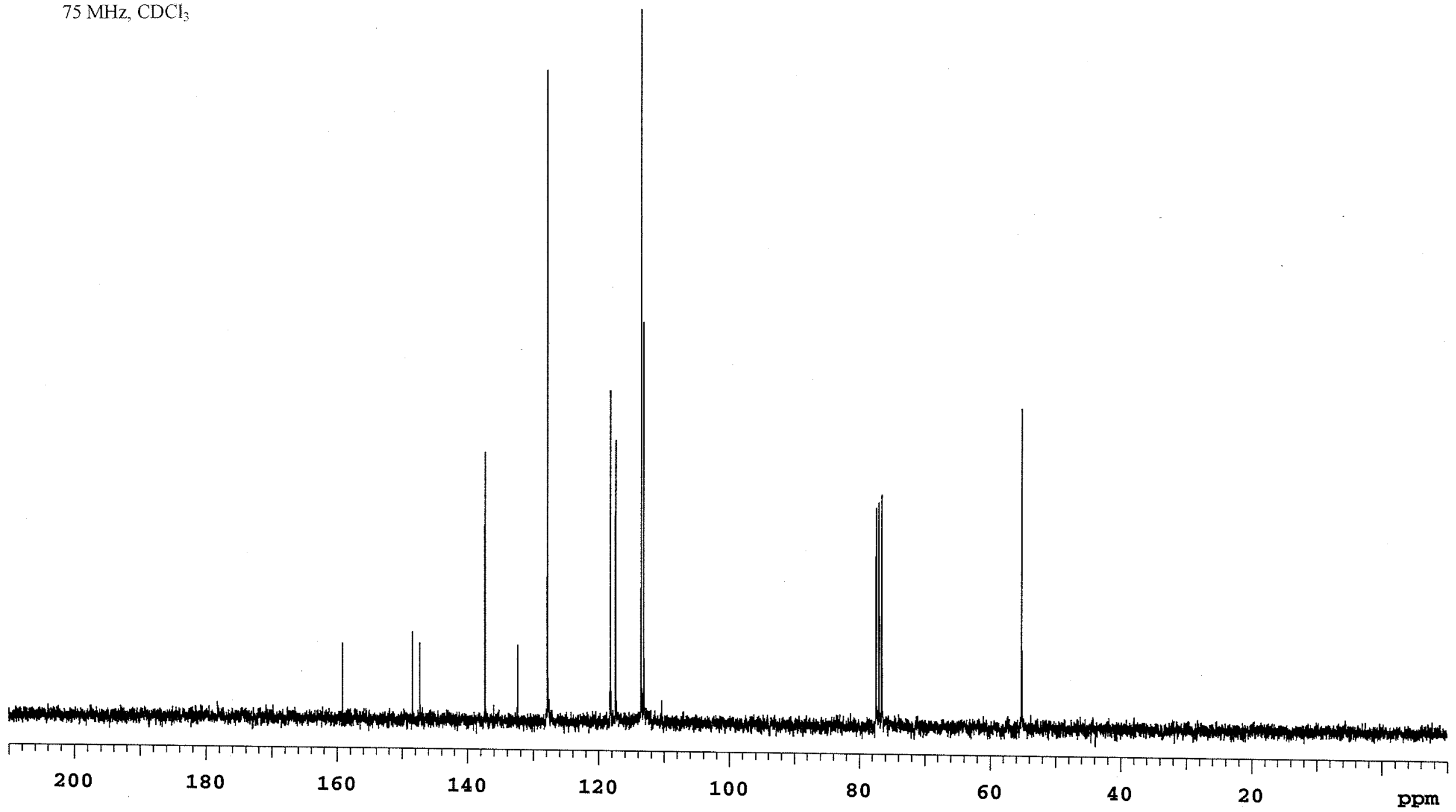

S56 
<smiles>C=CC(=C)C(=C)c1ccc([N+](=O)[O-])cc1</smiles>

$3 \mathfrak{e}$

$300 \mathrm{MHz}_{2} \mathrm{CDCl}_{3}$

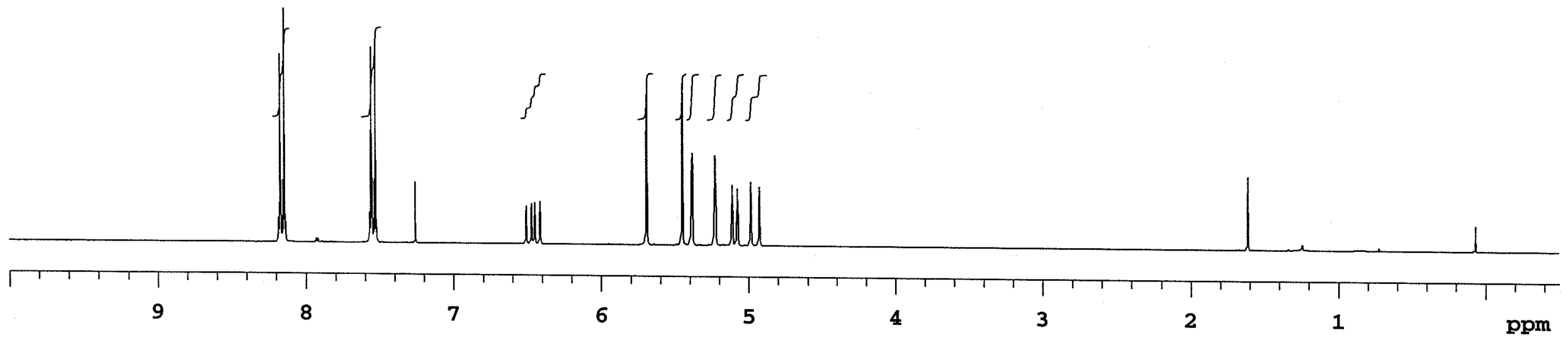

S57 


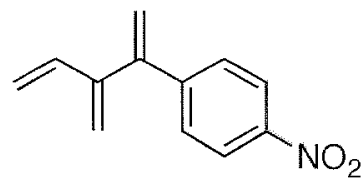

$3 \mathbf{e}$

$75 \mathrm{MHz}, \mathrm{CDCl}_{3}$

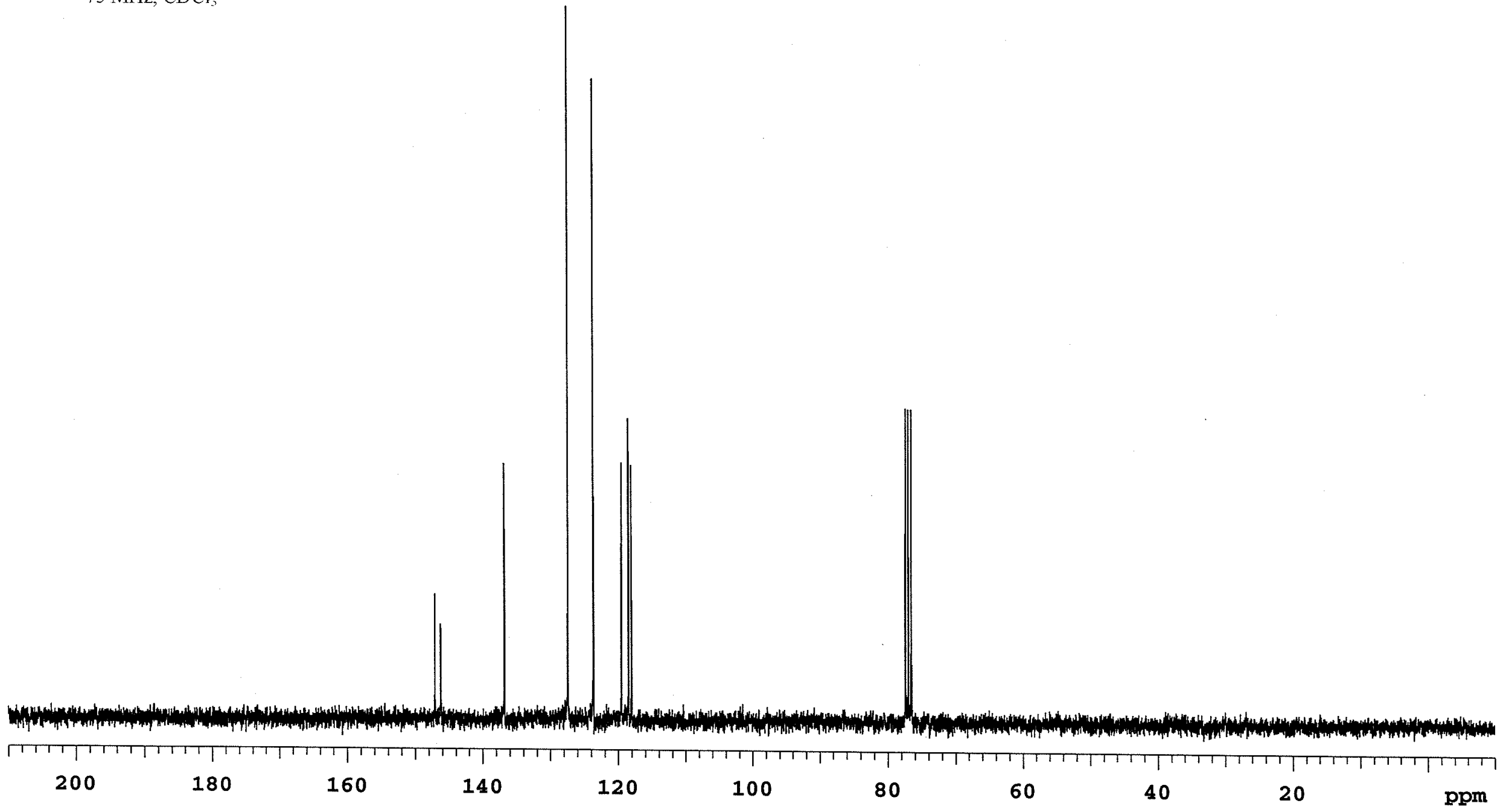


$\prod_{3 \mathbf{f}}^{1} \mathrm{OEt}$

$300 \mathrm{MHz}, \mathrm{CDCl}_{3}$

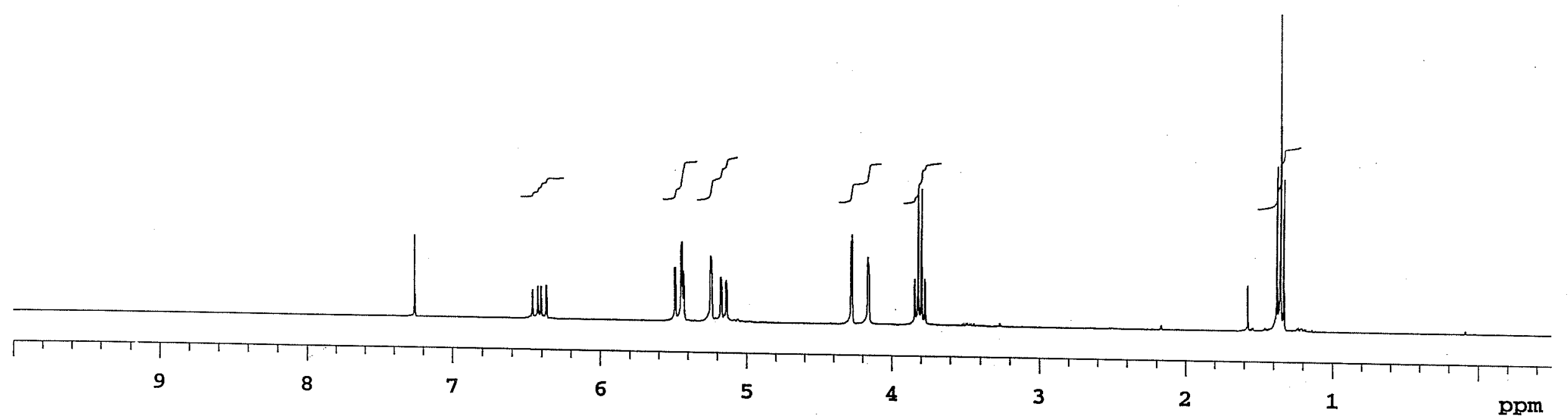




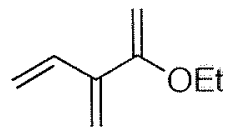

$3 f$

$75 \mathrm{MHz}_{2} \mathrm{CDCl}_{3}$

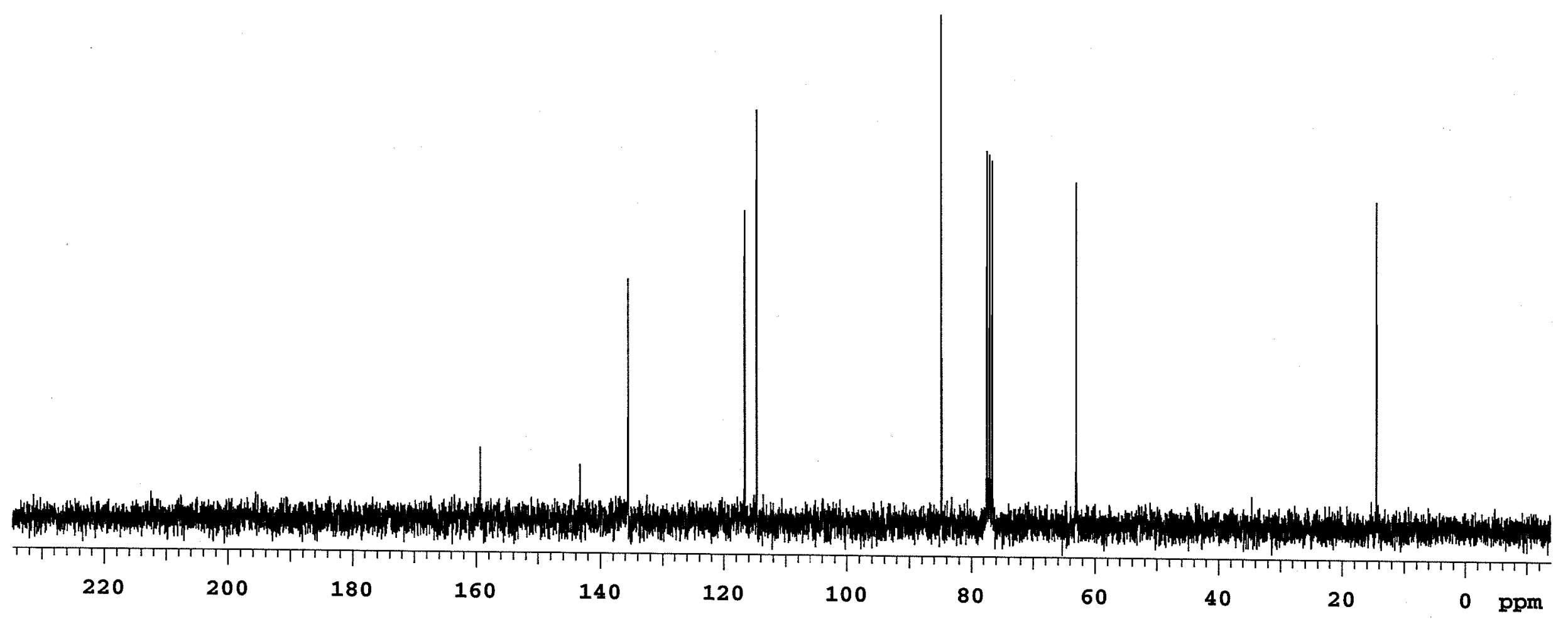




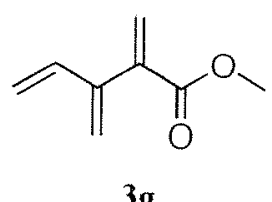

$300 \mathrm{MHz}_{3} \mathrm{CDCl}_{3}$

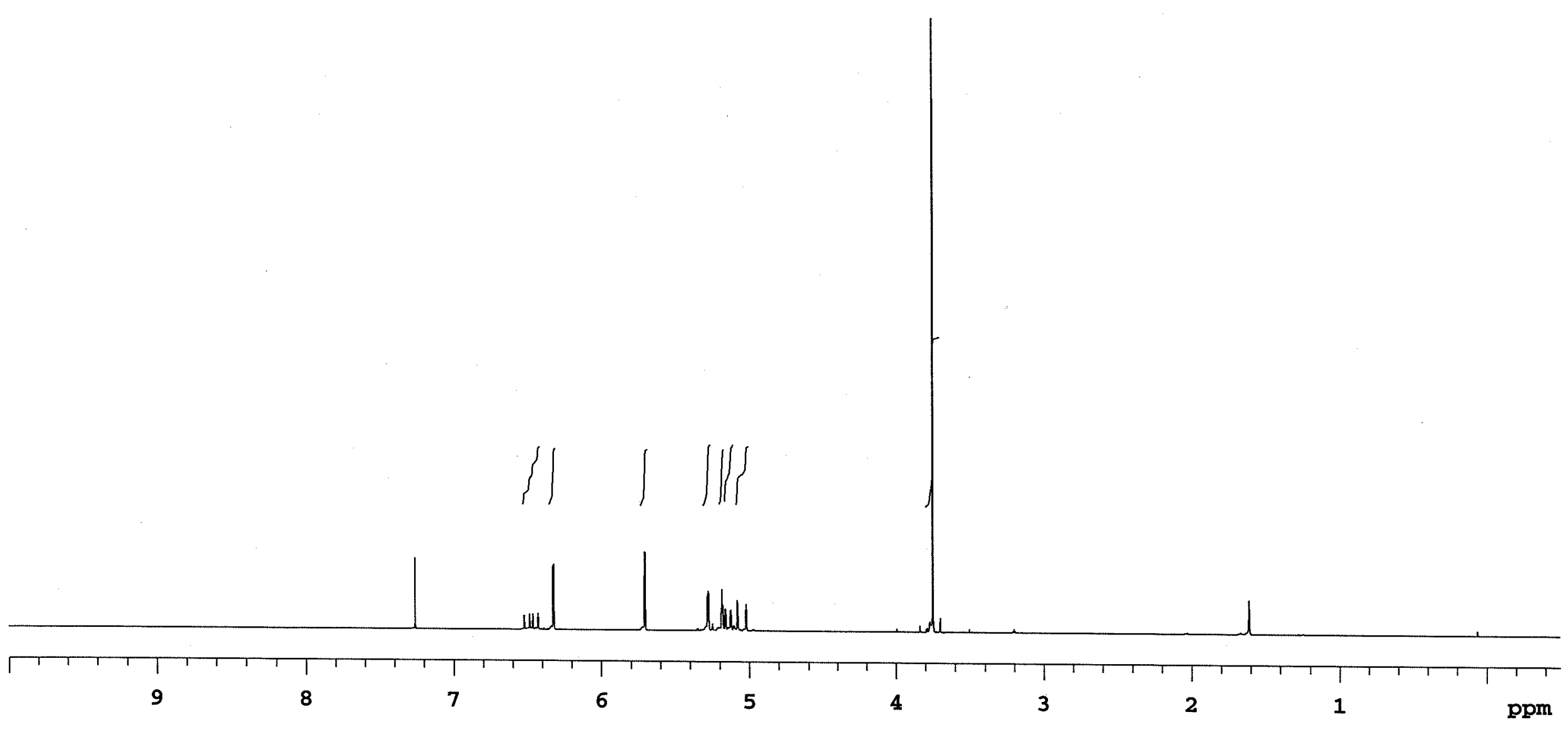




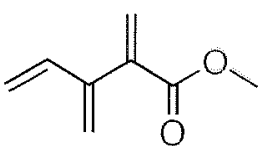

$3 g$

$75 \mathrm{MHz}, \mathrm{CDCl}_{5}$

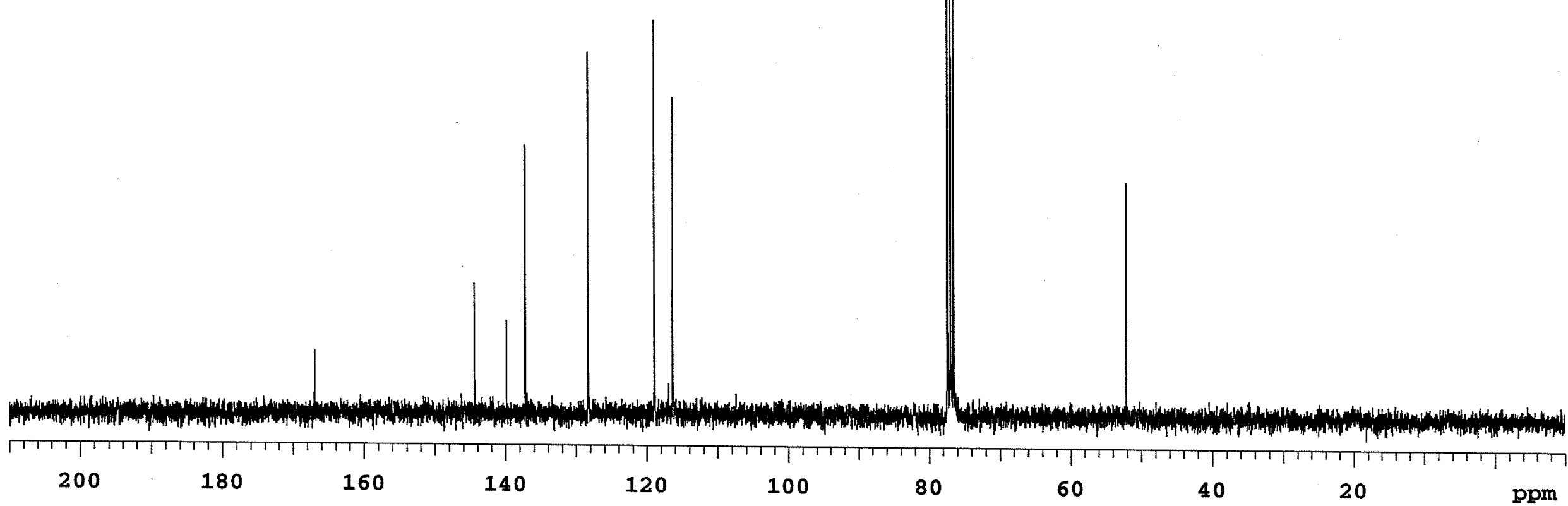




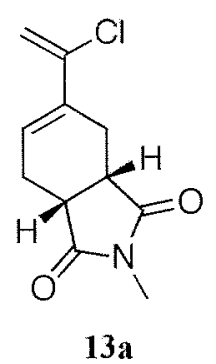

$300 \mathrm{MHz}_{2} \mathrm{CDCl}_{3}$

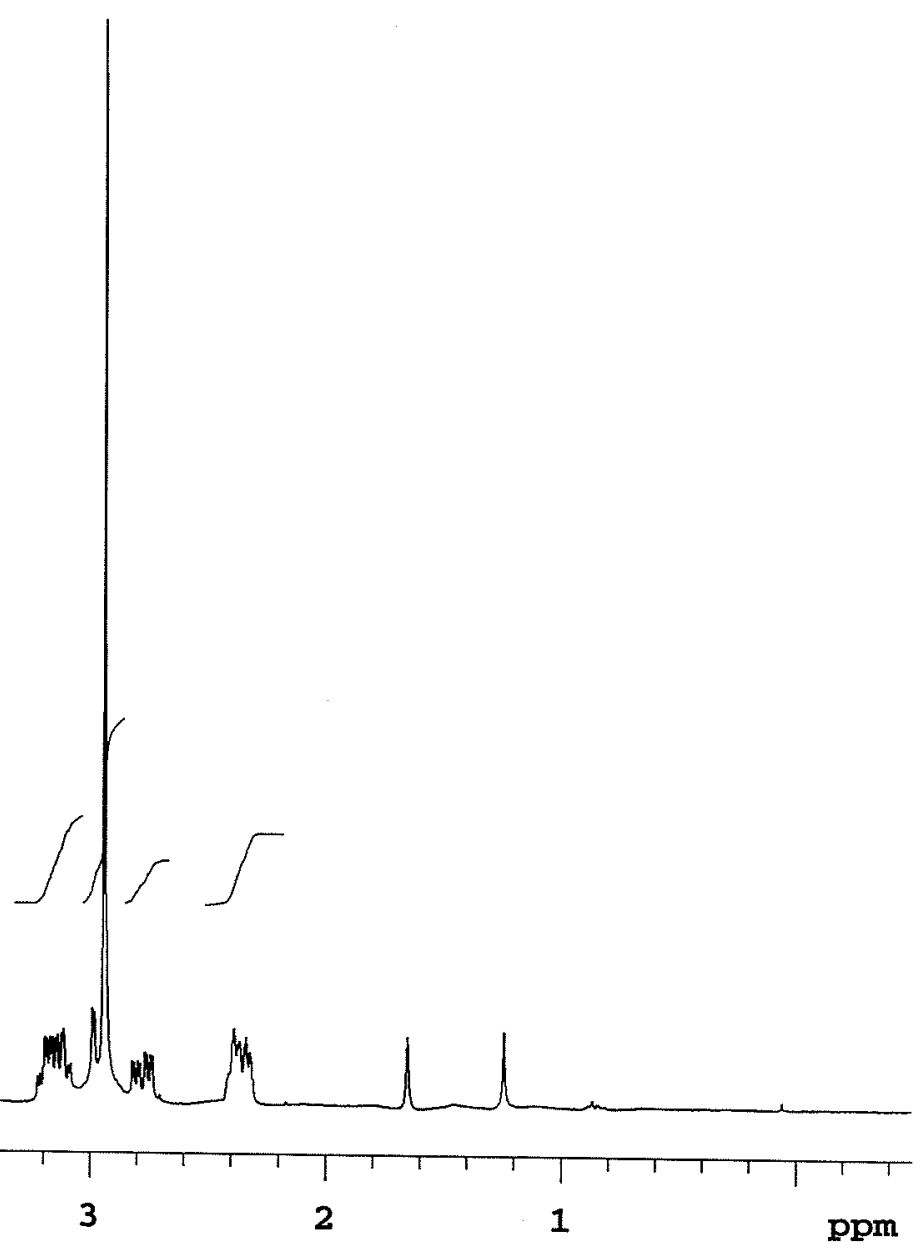




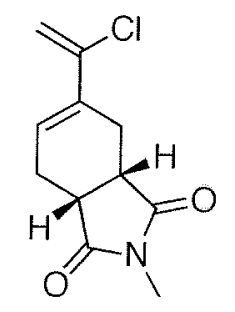

$13 a$

$125 \mathrm{MHz} \mathrm{CDCl}_{3}$

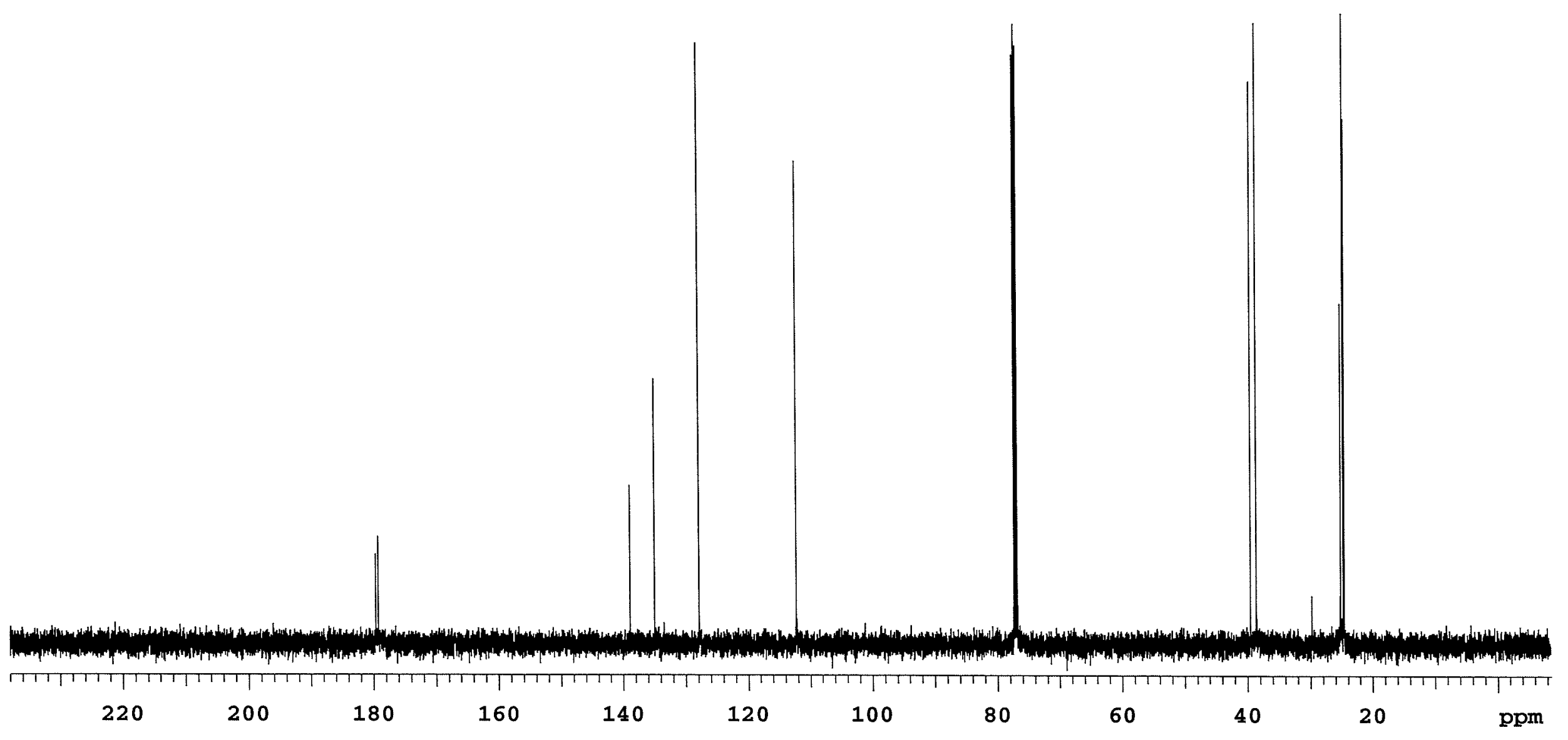




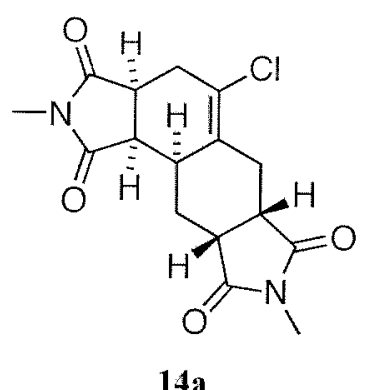

$300 \mathrm{MHz}, \mathrm{CDCl}_{3}$

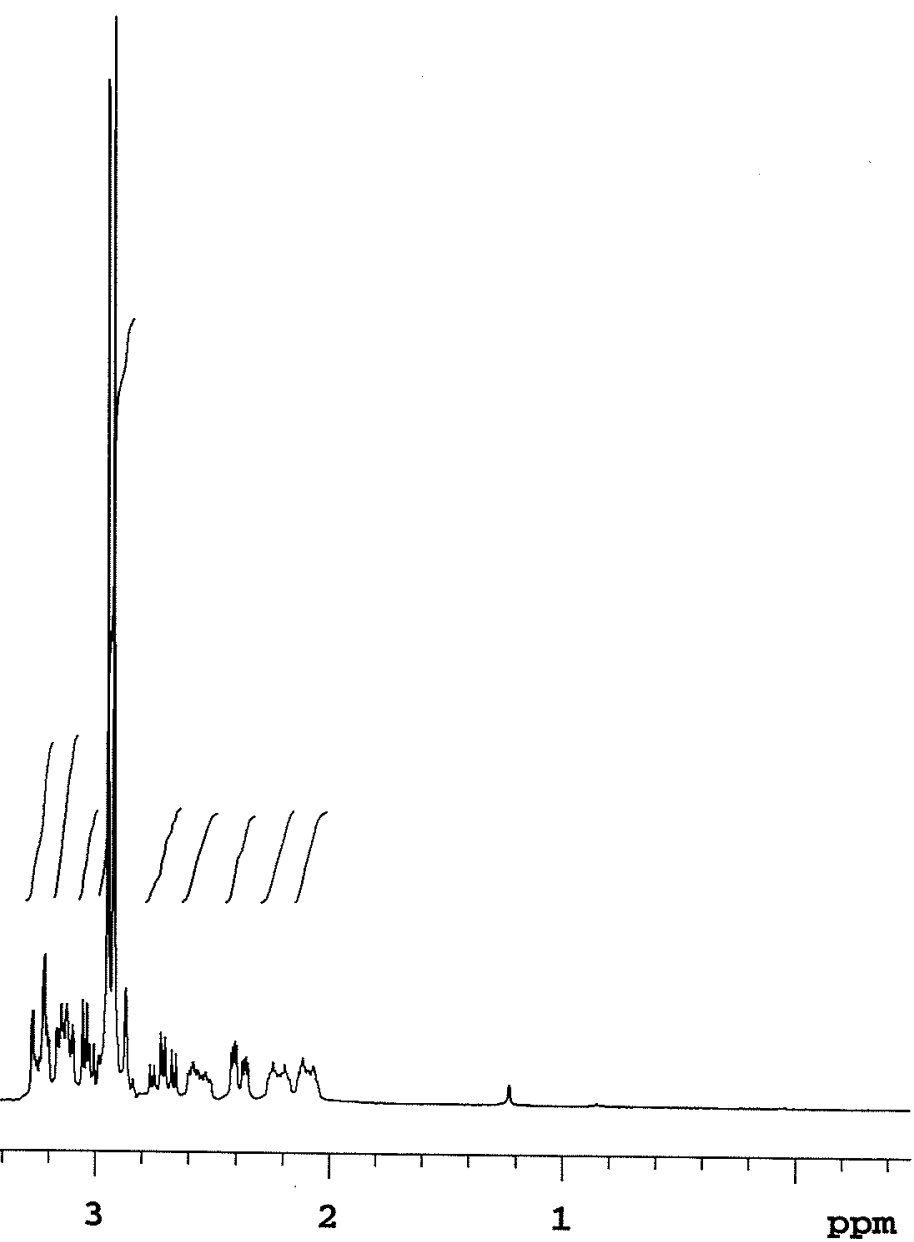




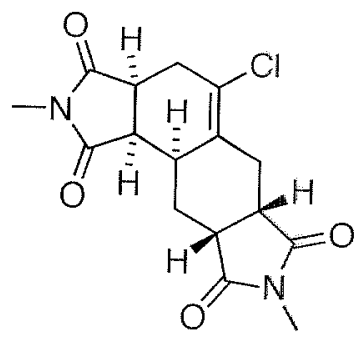

$14 a$

$75 \mathrm{MHz}, \mathrm{CDCl}_{3}$

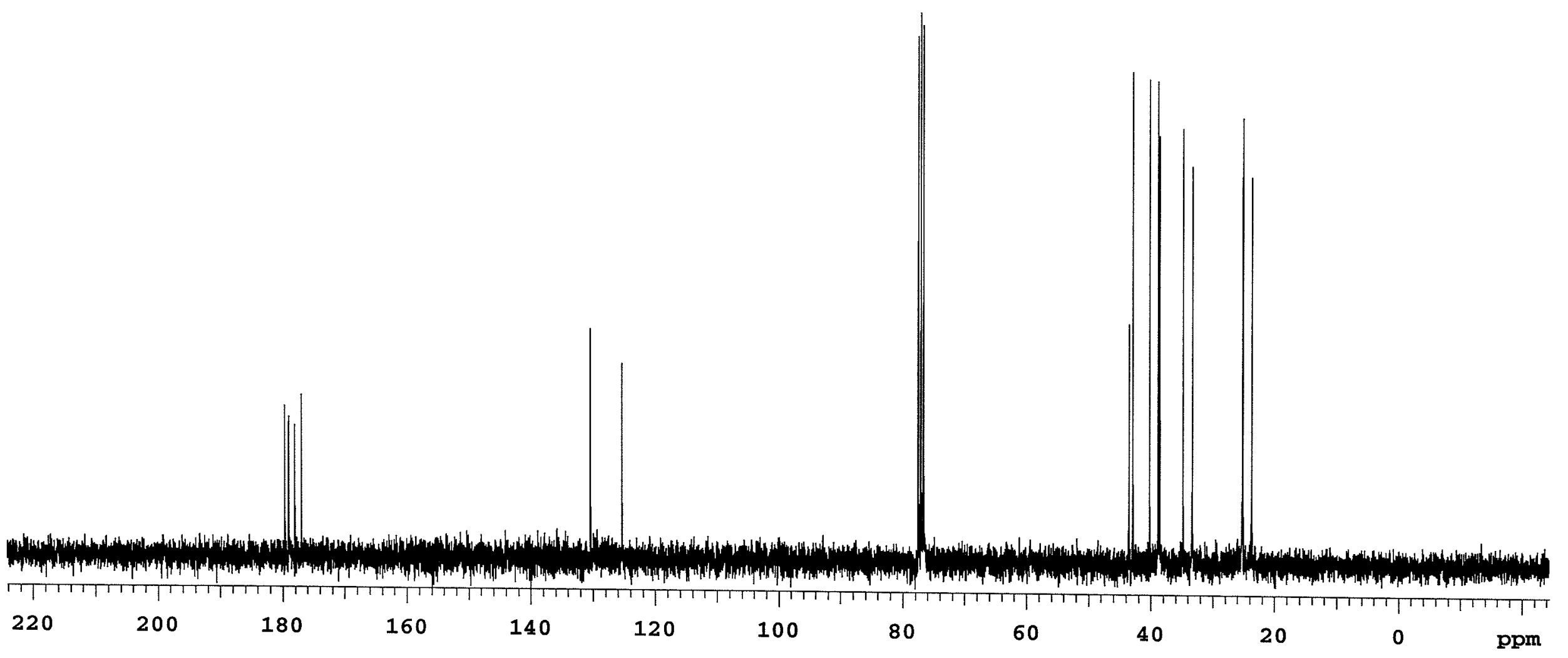




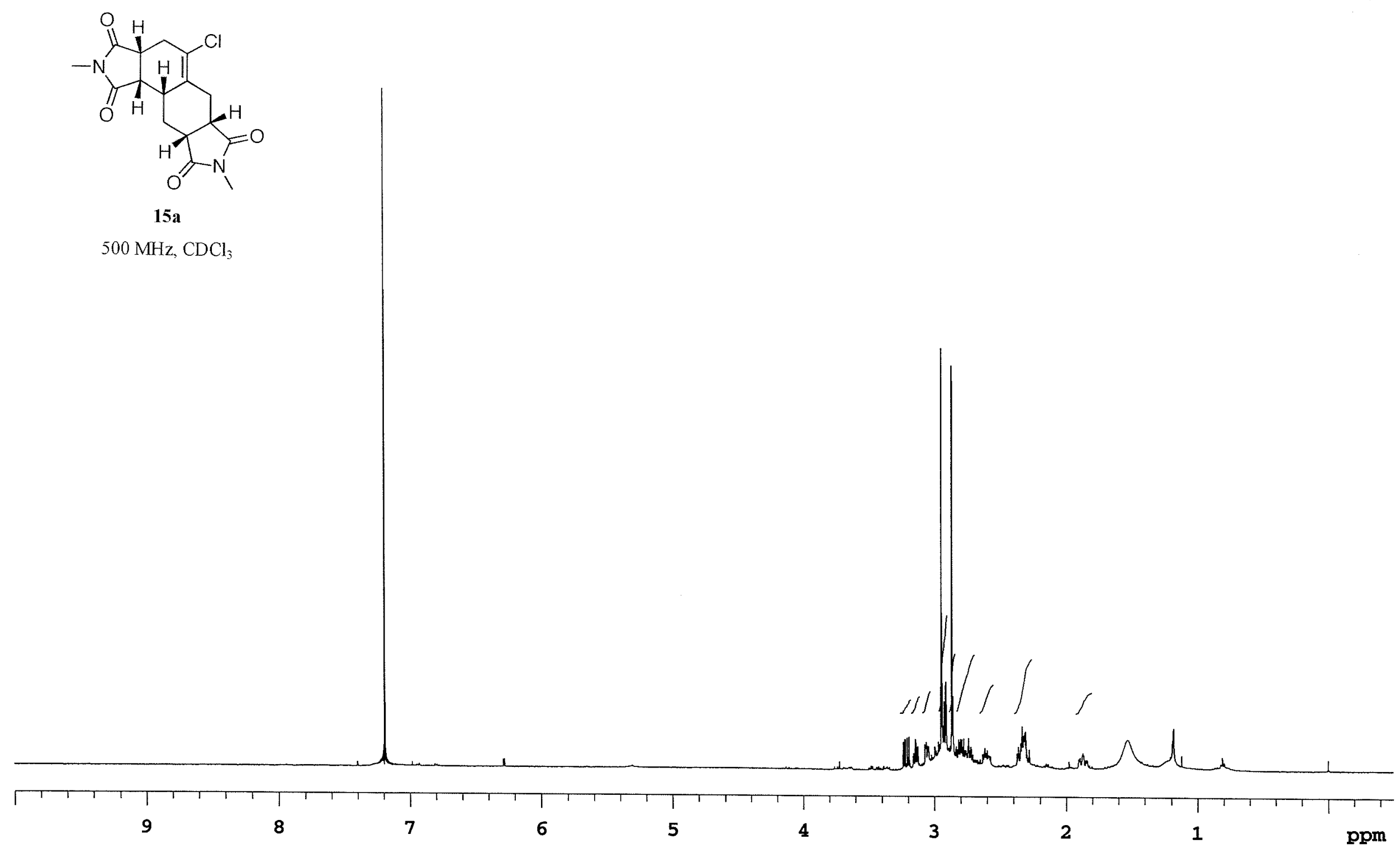




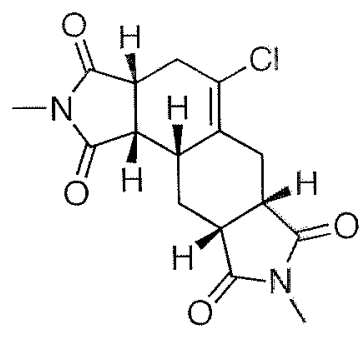

$15 a$

$125 \mathrm{MHz}, \mathrm{CDCl}_{3}$

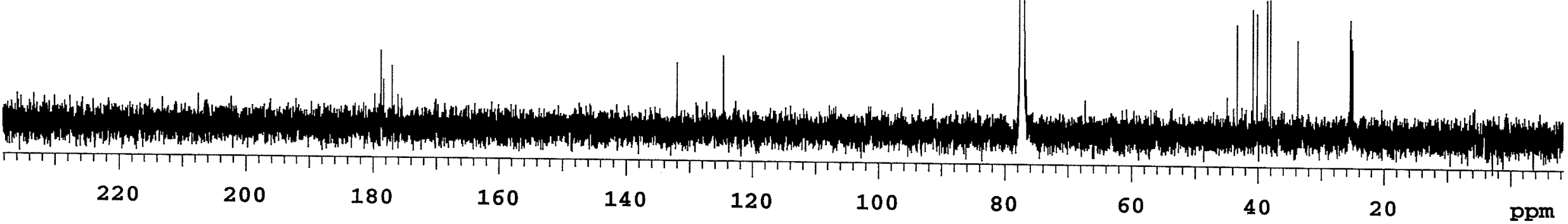




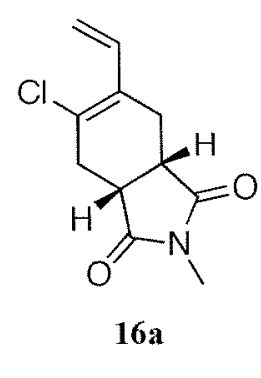

$300 \mathrm{MHz}_{2} \mathrm{CDCl}_{3}$

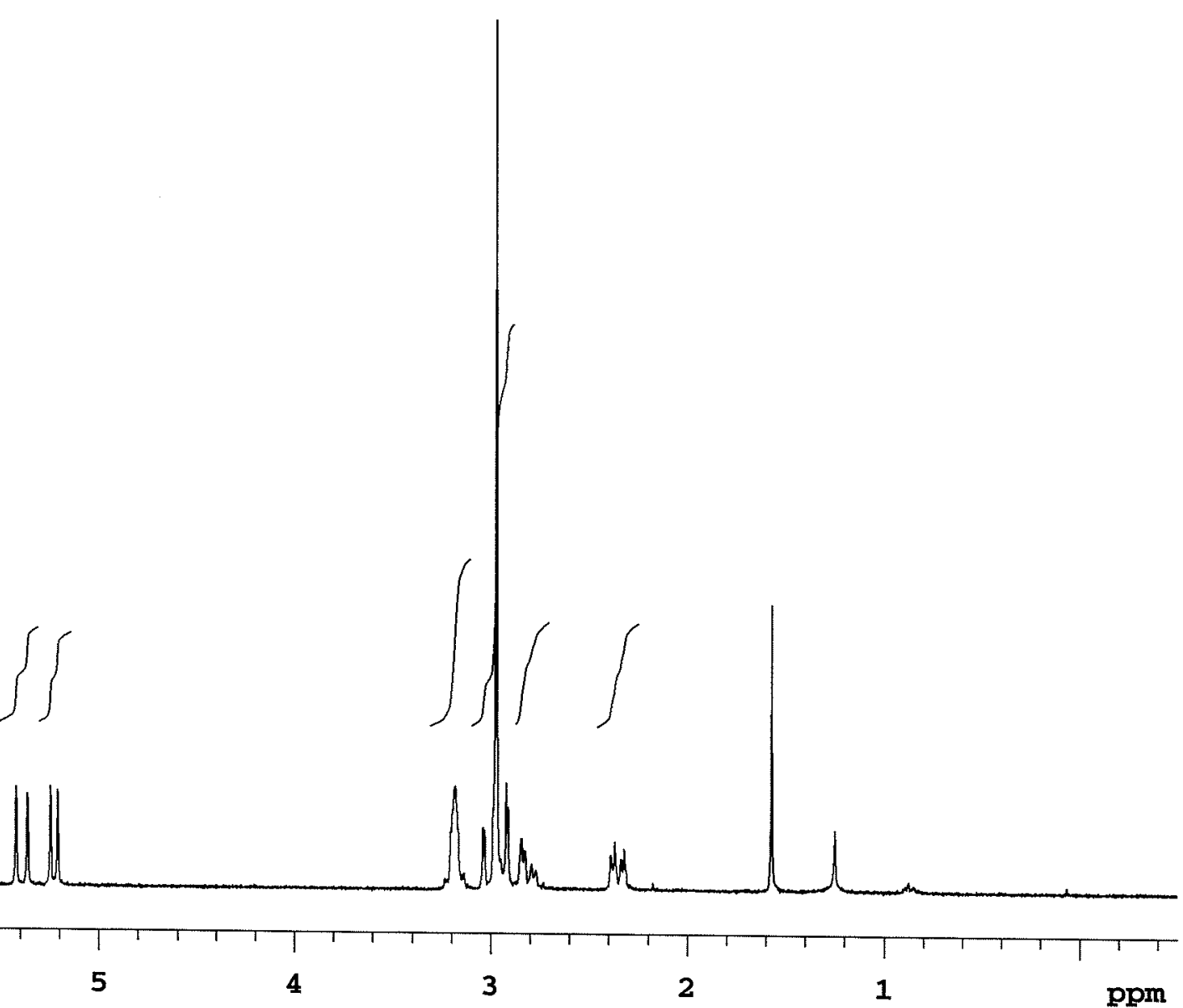




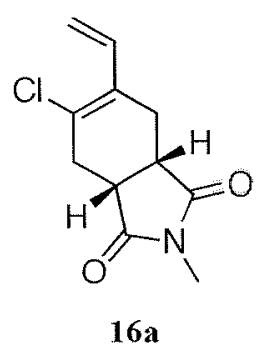

$125 \mathrm{MHz} \mathrm{CDCl}_{3}$

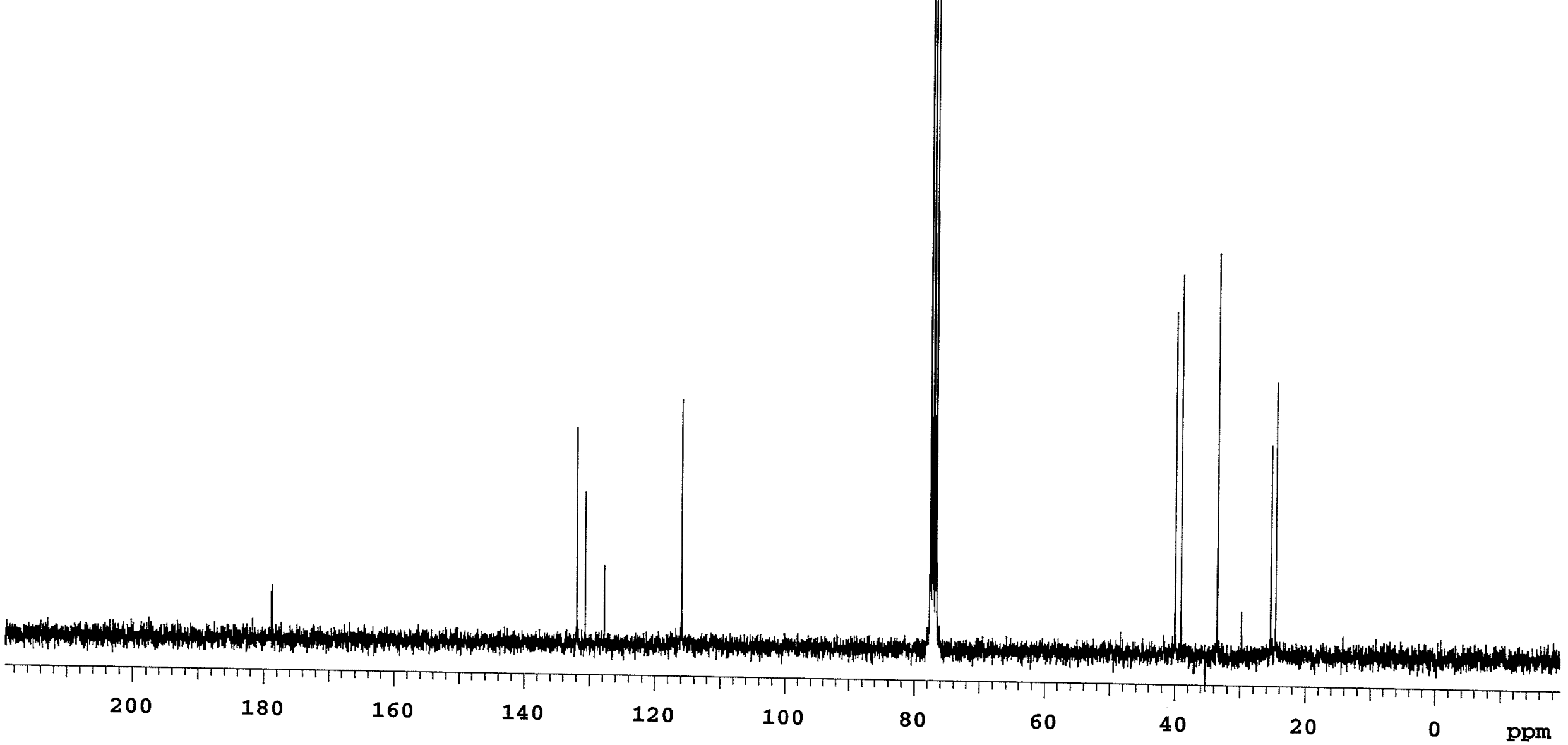




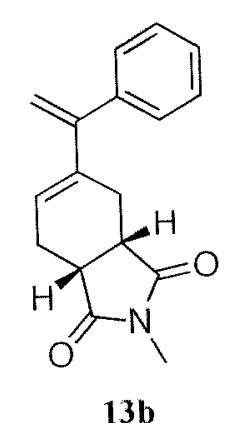

$300 \mathrm{MHz}, \mathrm{CDCl}_{3}$

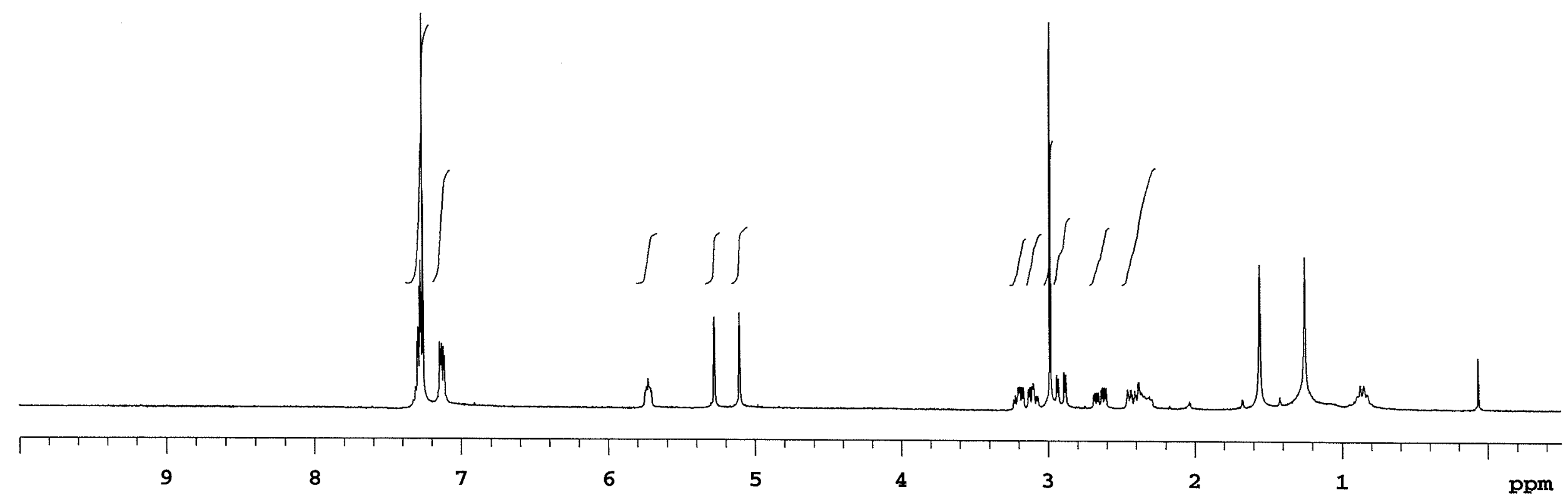




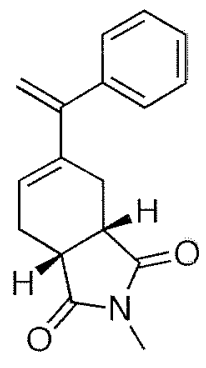

13b

$125 \mathrm{MHz} \mathrm{CDCl}_{3}$

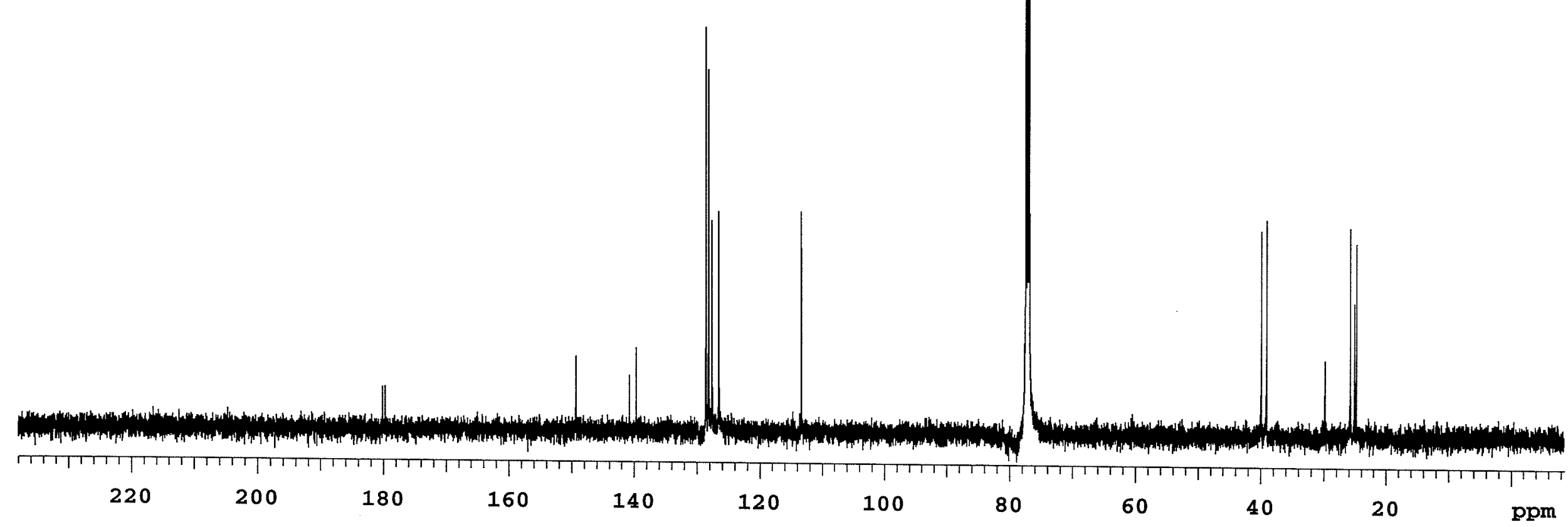




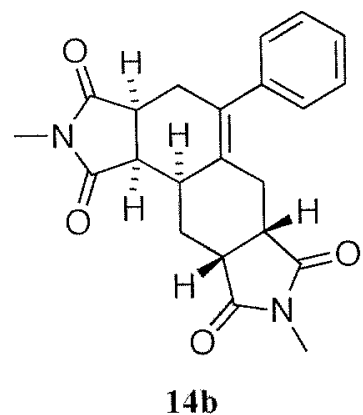

$500 \mathrm{MHz}, \mathrm{CDCl}_{3}$

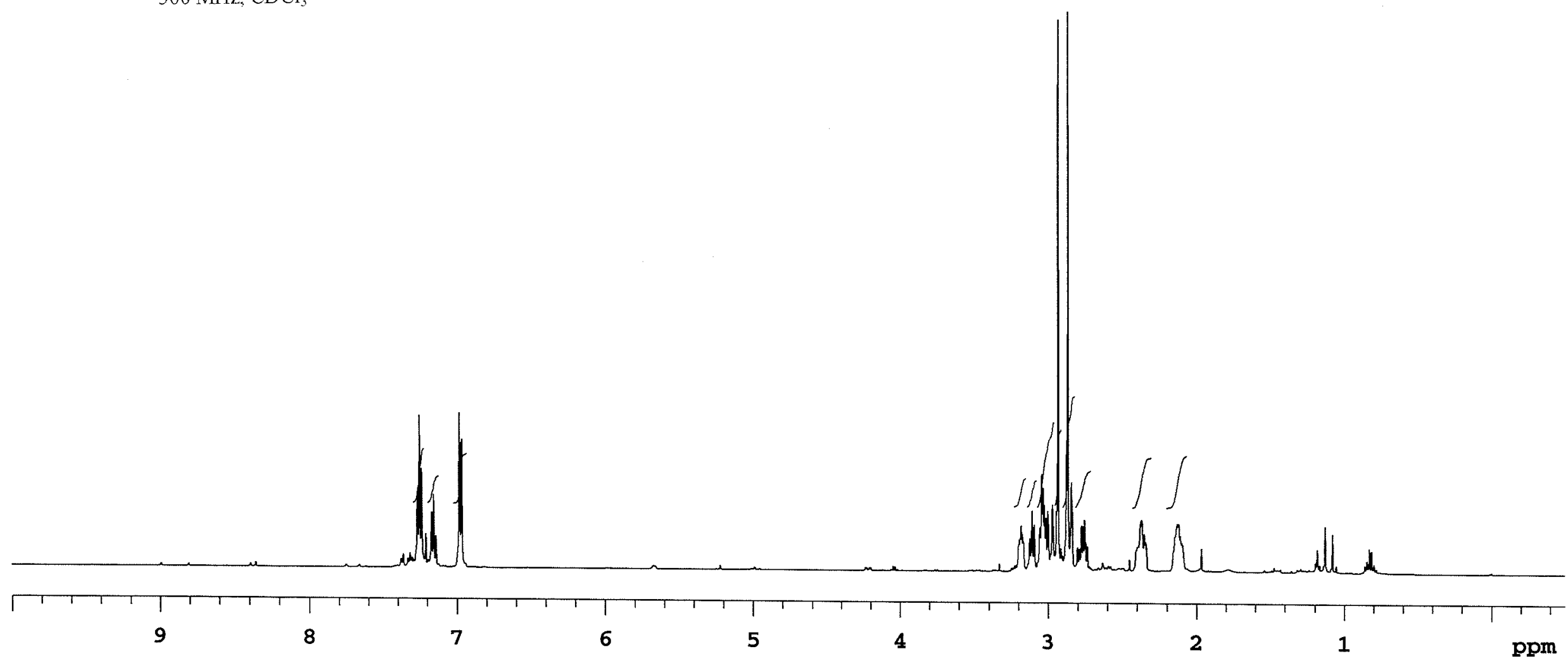




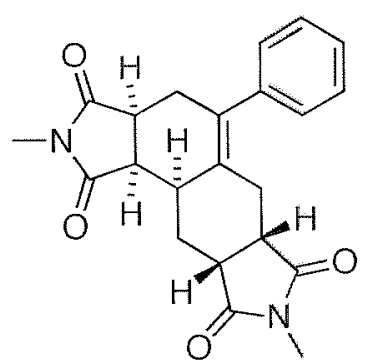

14b

$125 \mathrm{MHz} \mathrm{CDCl}_{3}$

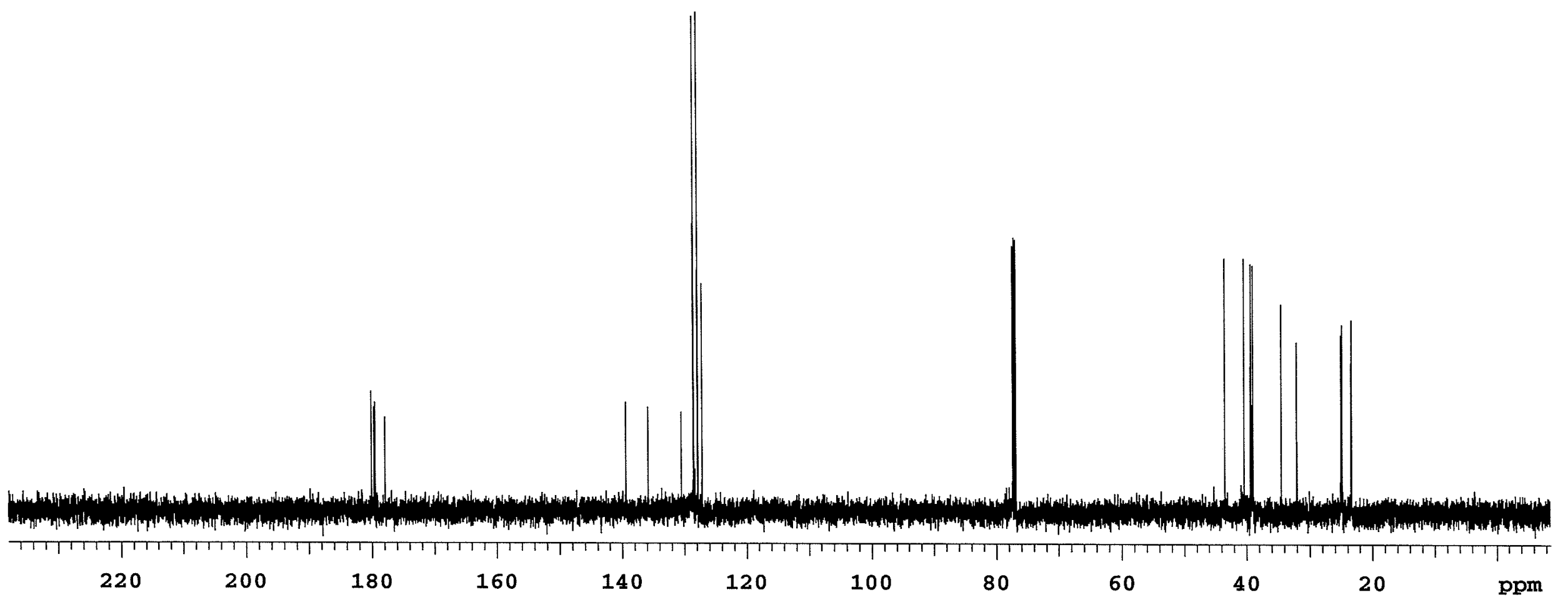




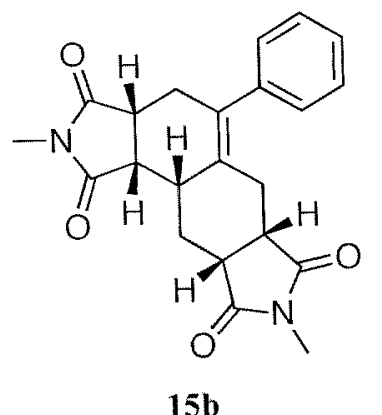

$500 \mathrm{MHz}, \mathrm{CDCl}_{3}$

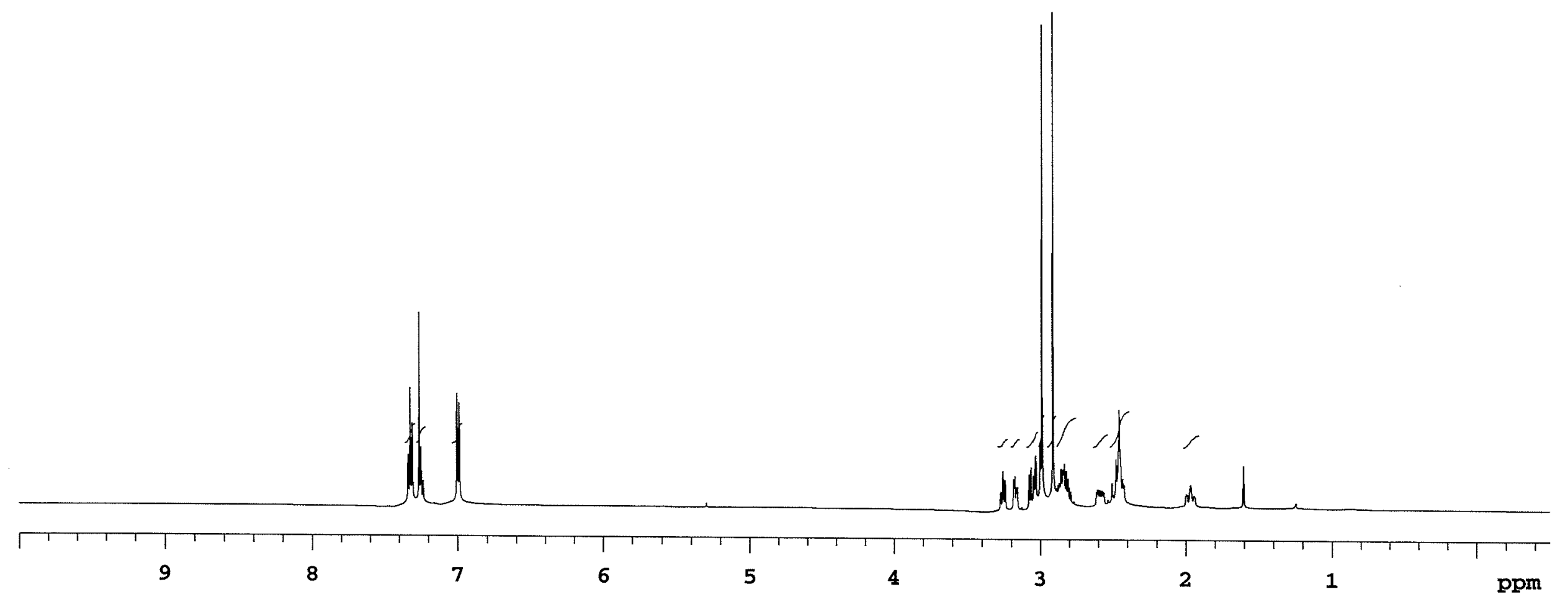




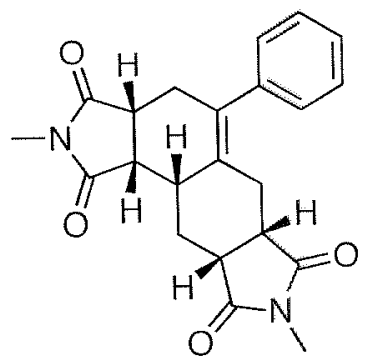

$15 \mathrm{~b}$

$125 \mathrm{MHz}_{2} \mathrm{CDCl}_{3}$

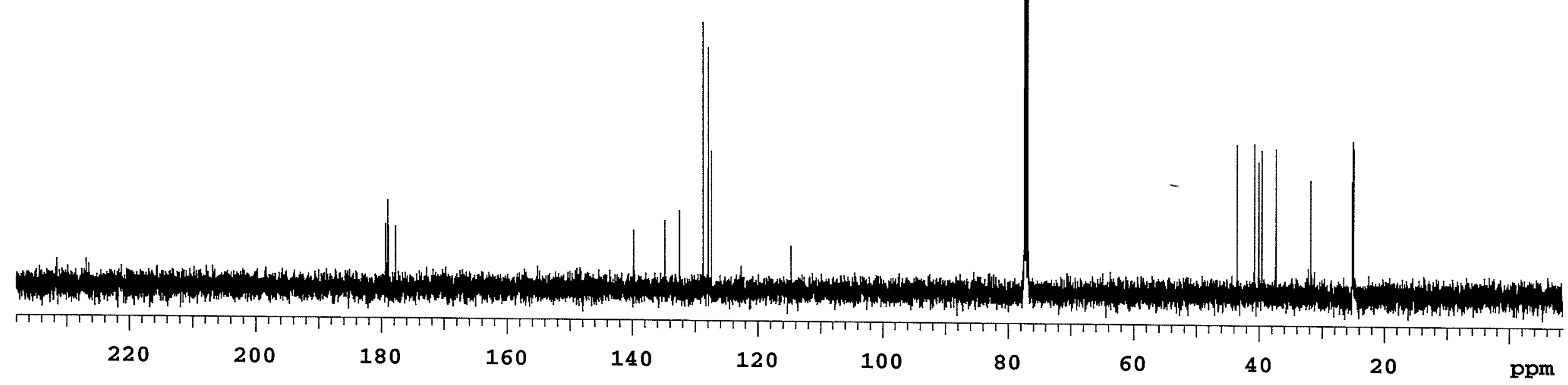




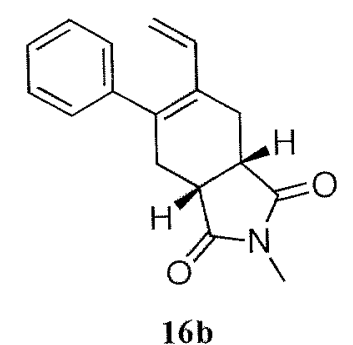

$500 \mathrm{MHz}_{2} \mathrm{CDCl}_{3}$

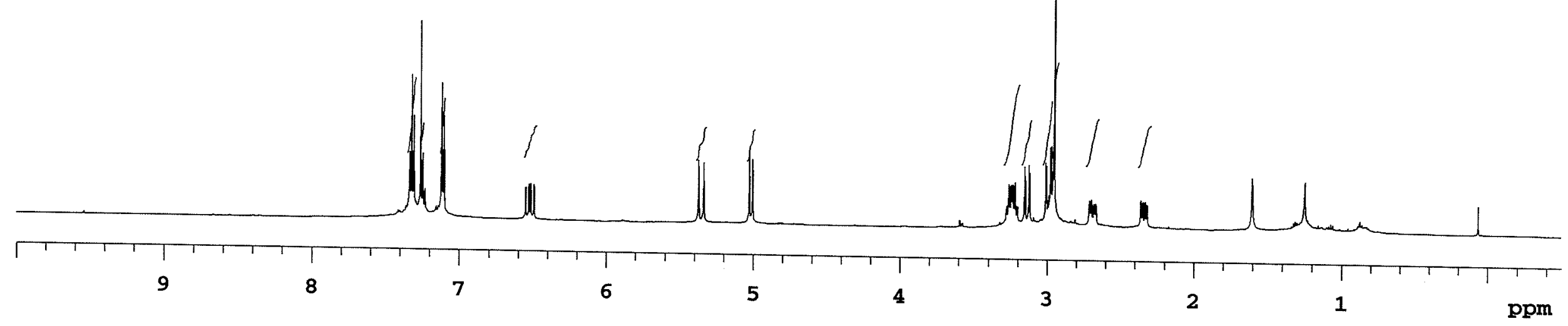




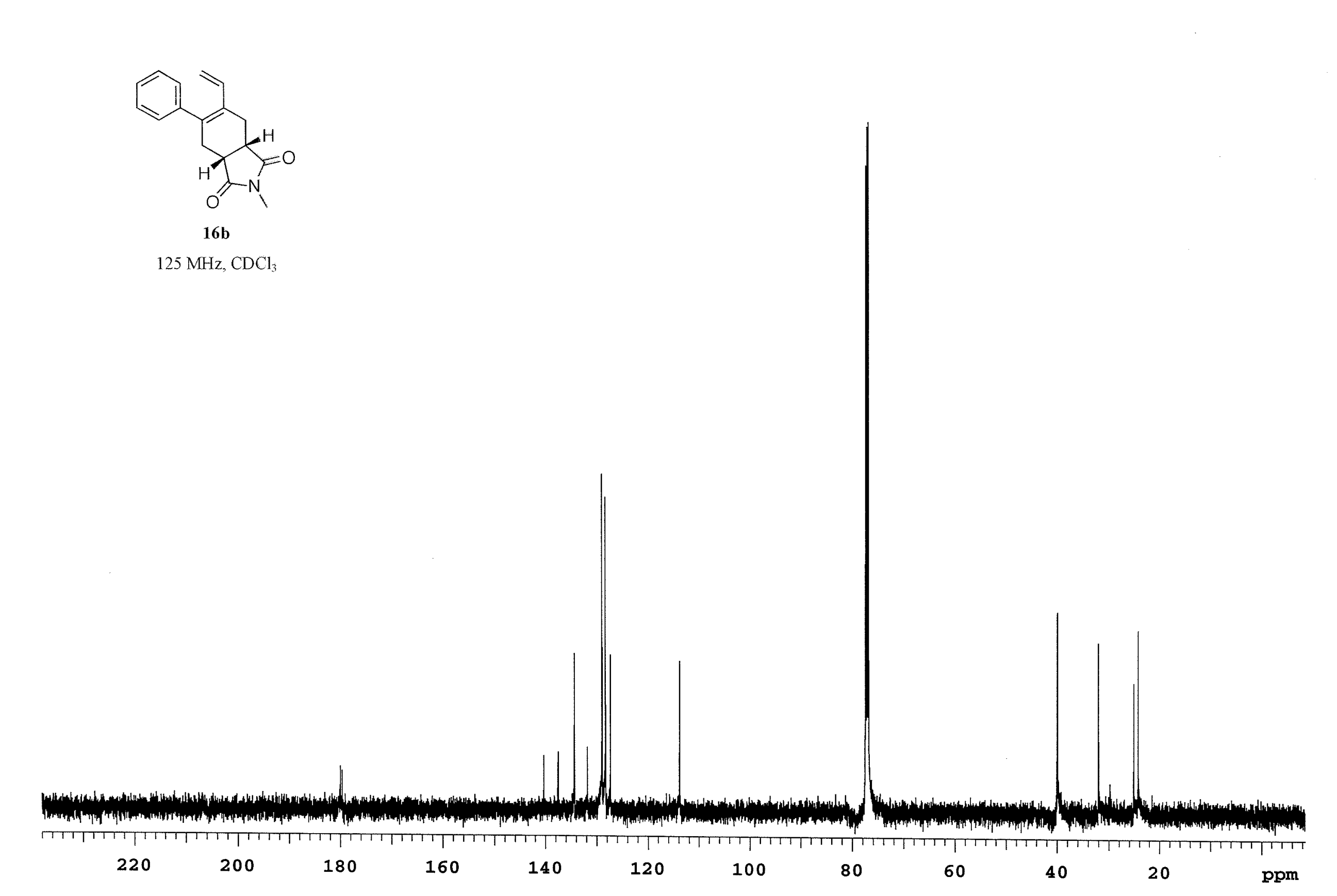




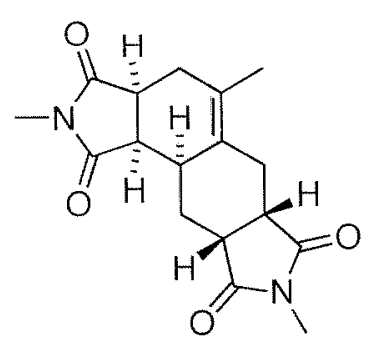

$14 \mathrm{c}$

$500 \mathrm{MHz}_{2} \mathrm{CDCl}_{3}$

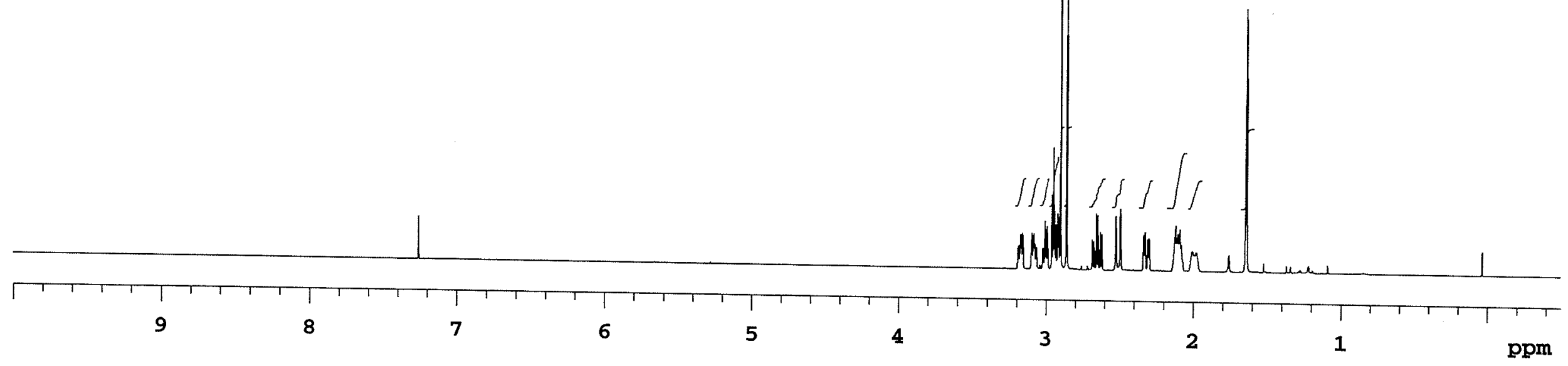




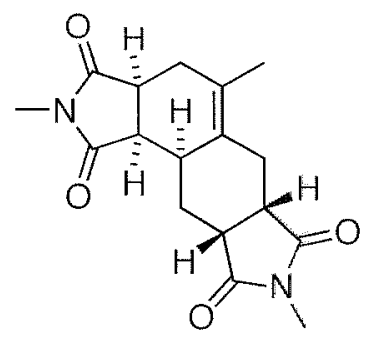

$14 \mathrm{c}$

$125 \mathrm{MHz} \mathrm{CDCl}_{3}$

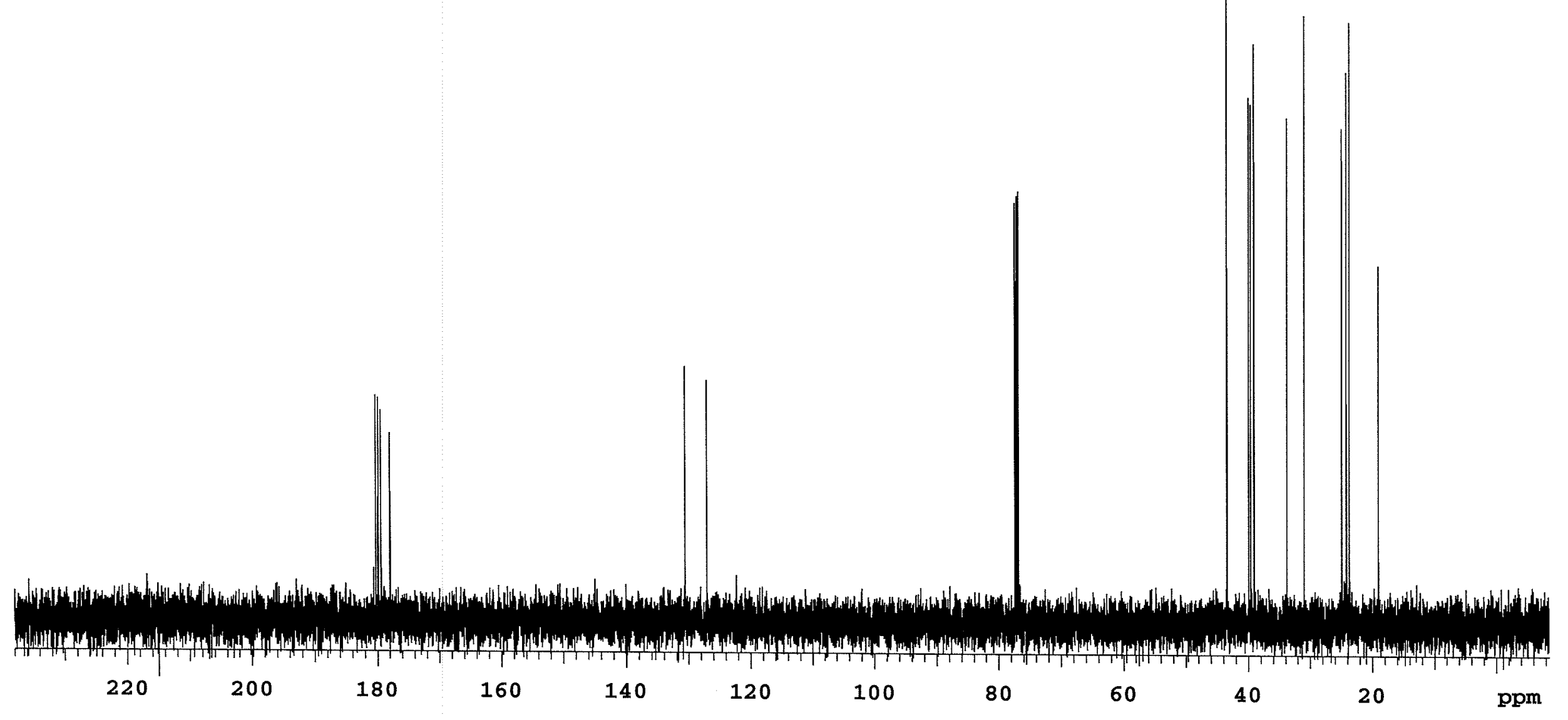




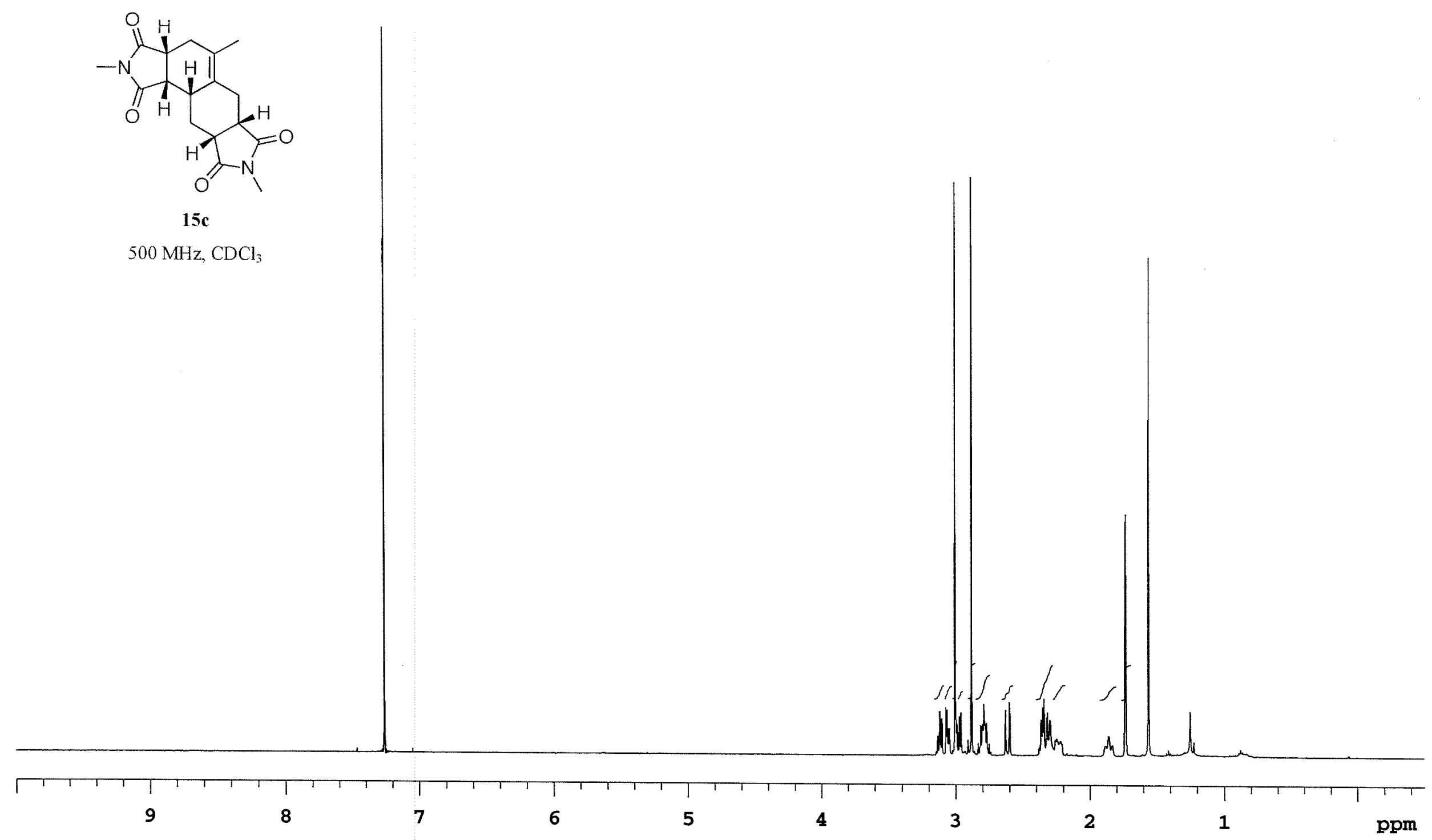




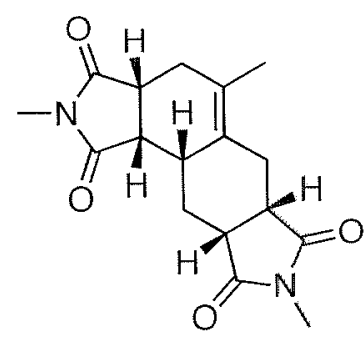

$15 \mathrm{c}$

$125 \mathrm{MHz}_{2} \mathrm{CDCl}_{3}$

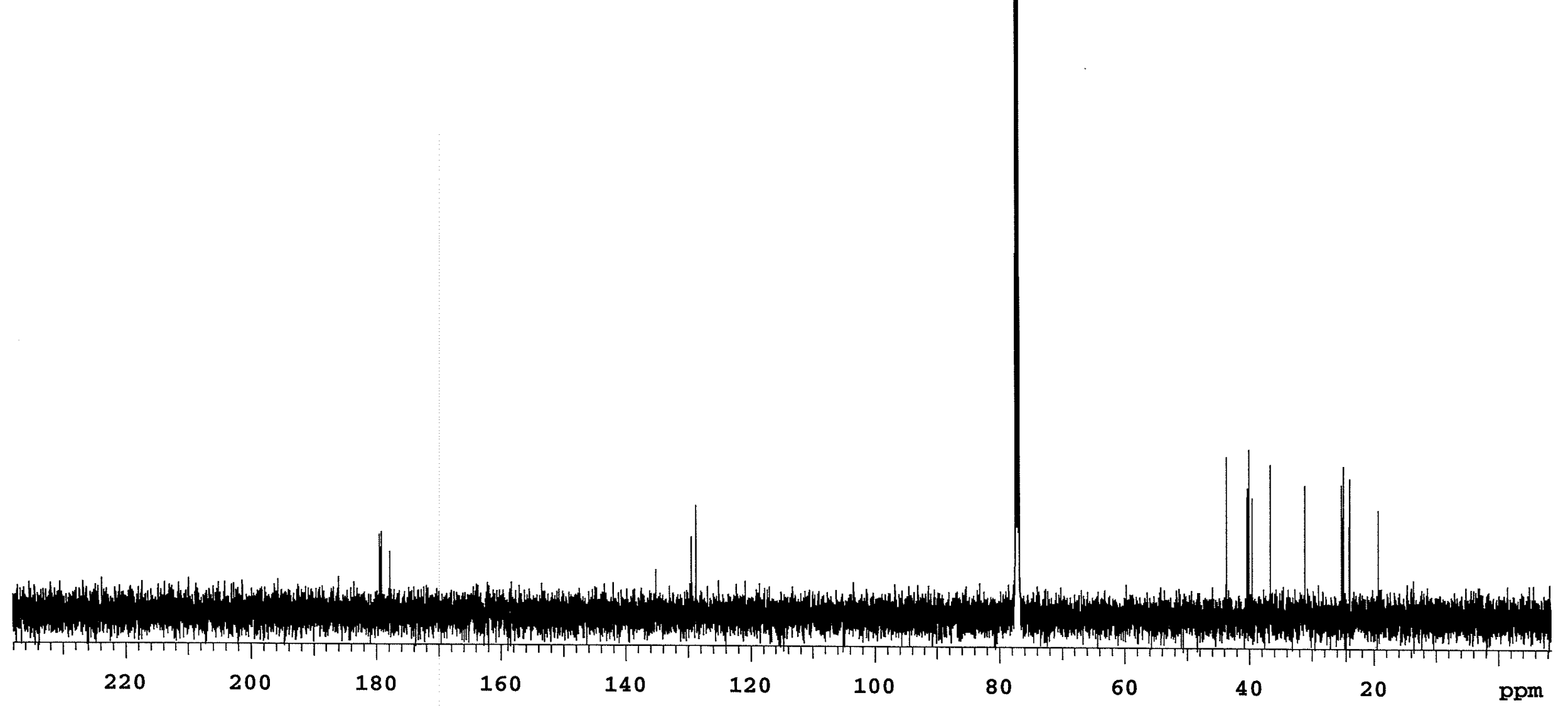




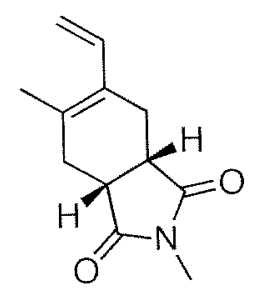

$16 \mathrm{c}$

$500 \mathrm{HMz}_{3} \mathrm{CDCl}_{3}$

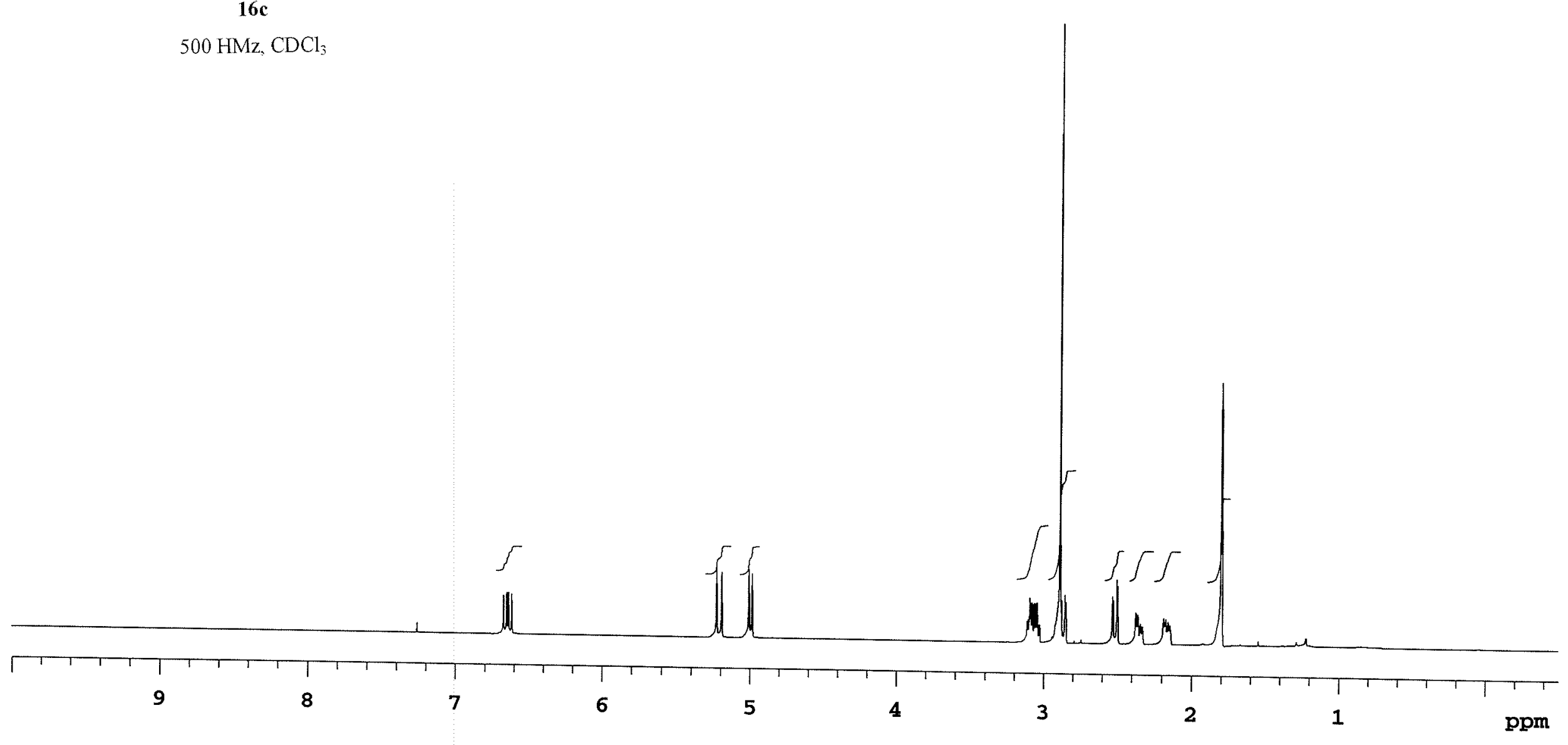




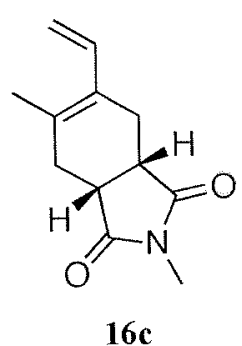

$125 \mathrm{HMz} \mathrm{CDCl}_{3}$

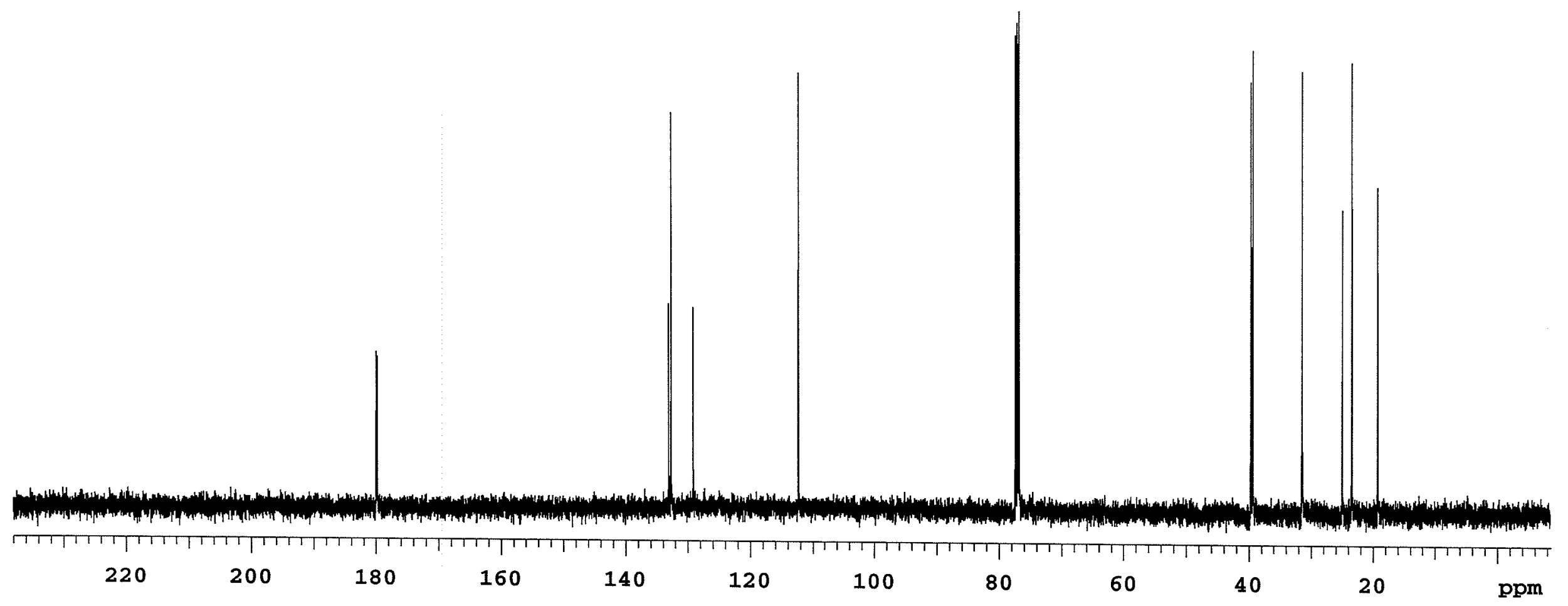




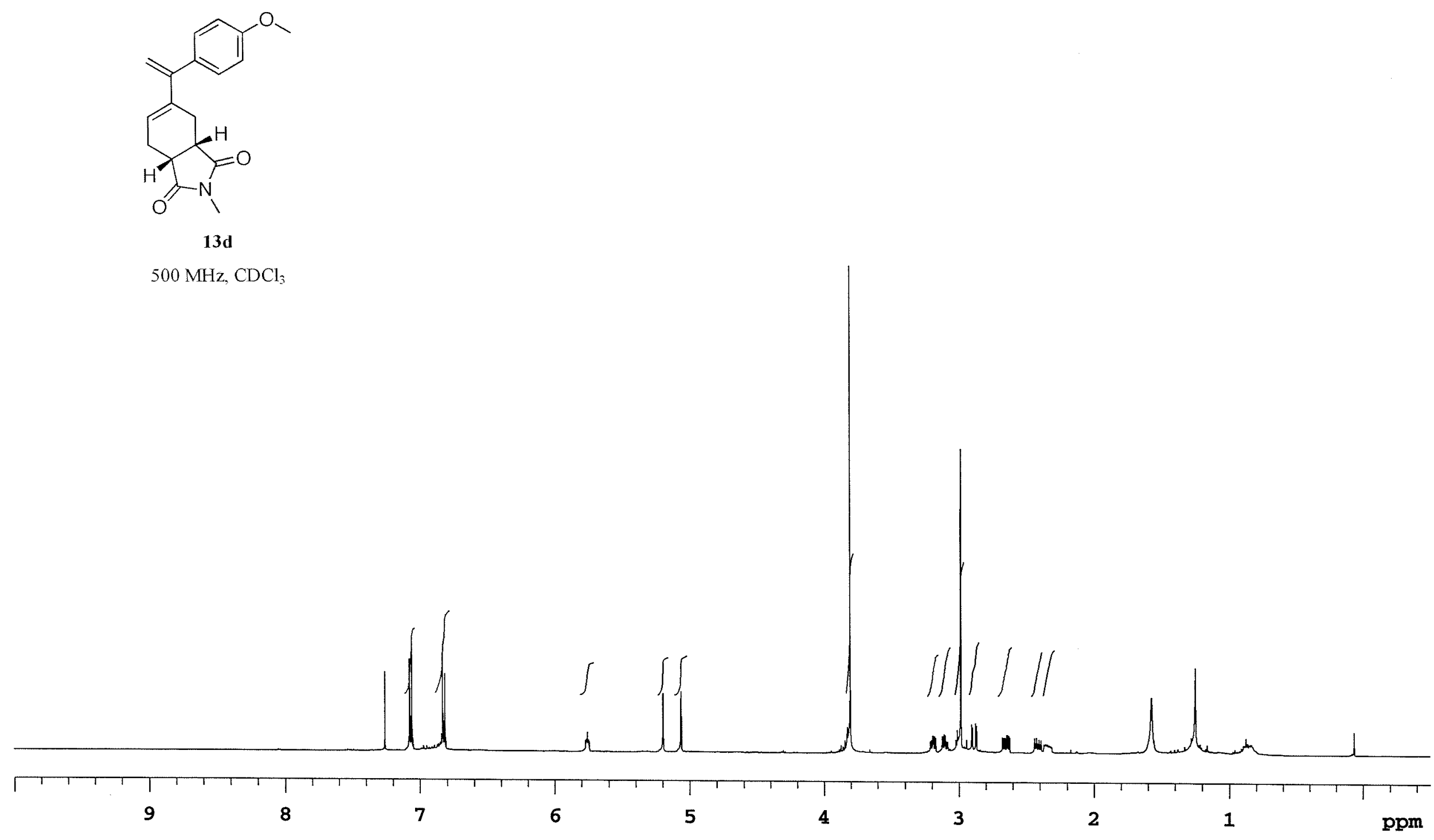




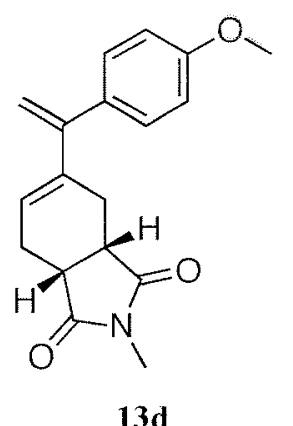

$125 \mathrm{MHz}_{2} \mathrm{CDCl}_{3}$

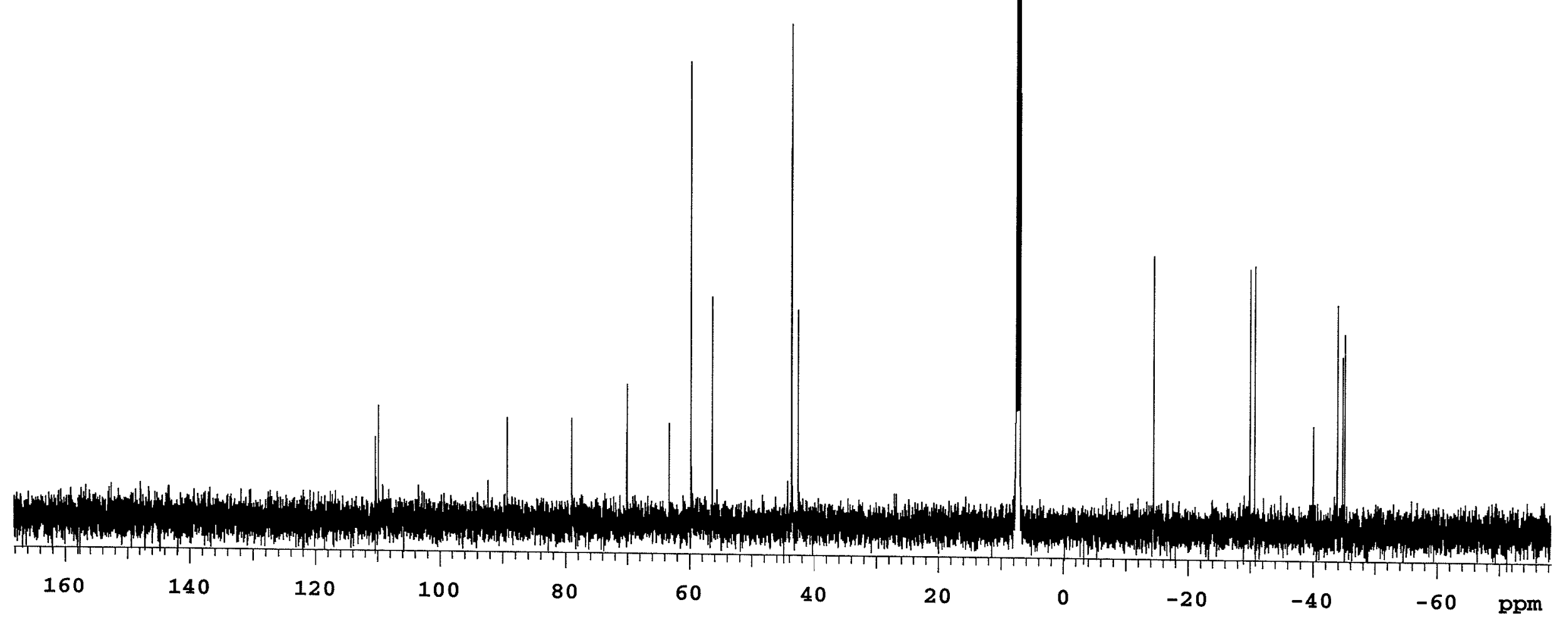




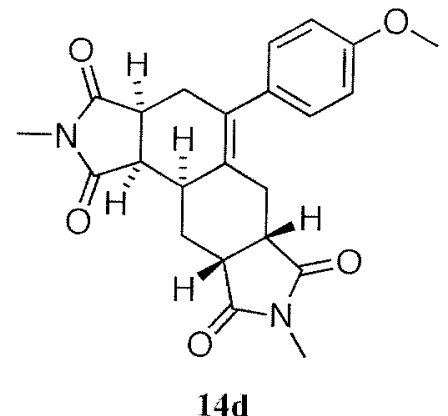

$600 \mathrm{MHz}, \mathrm{CDCl}_{3}$

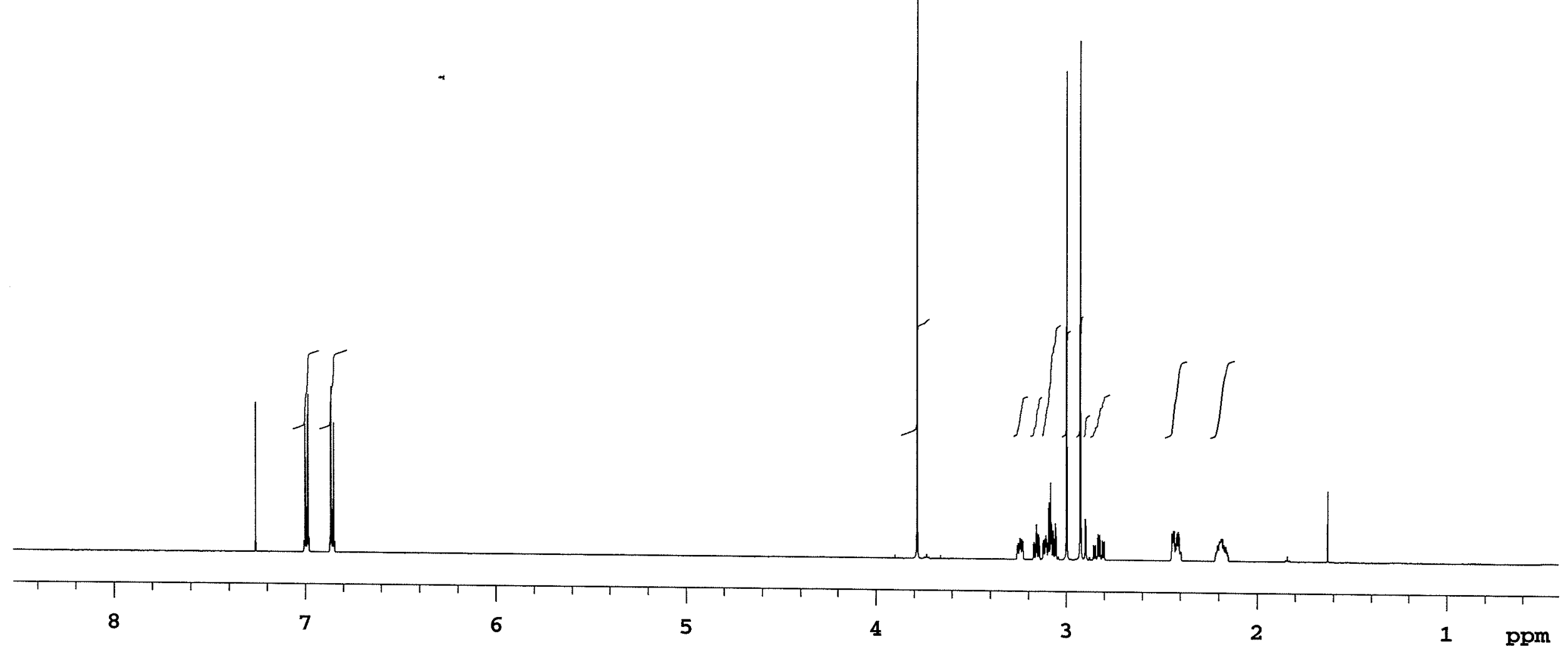




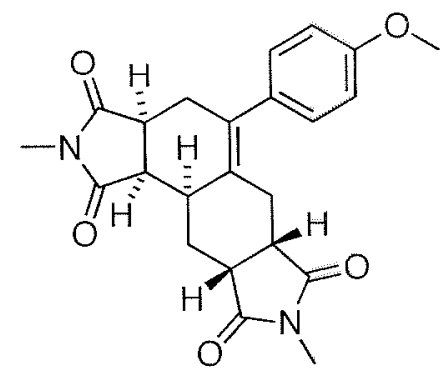

$14 d$

$75 \mathrm{HMz}_{2} \mathrm{CDCl}_{3}$

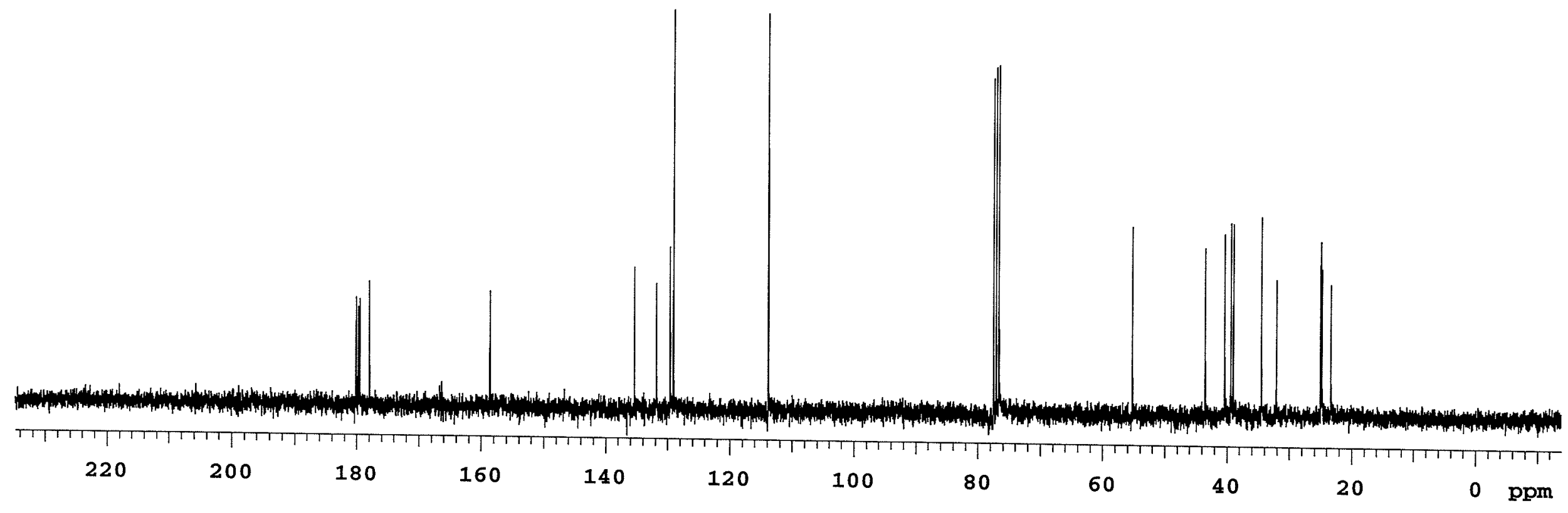




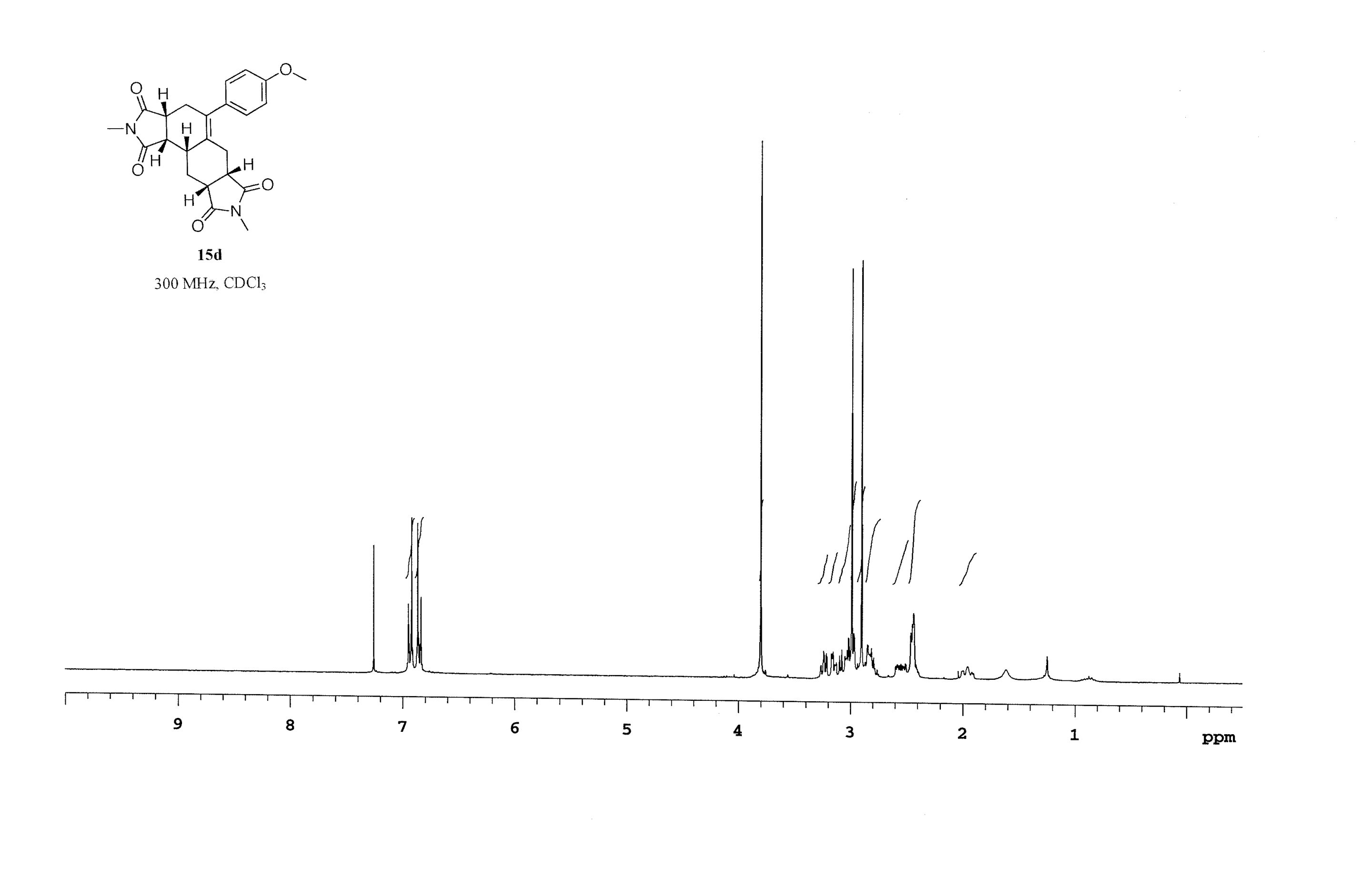




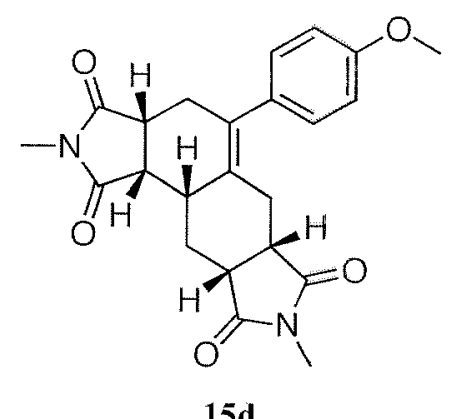

$75 \mathrm{MHz}_{2} \mathrm{CDCl}_{3}$

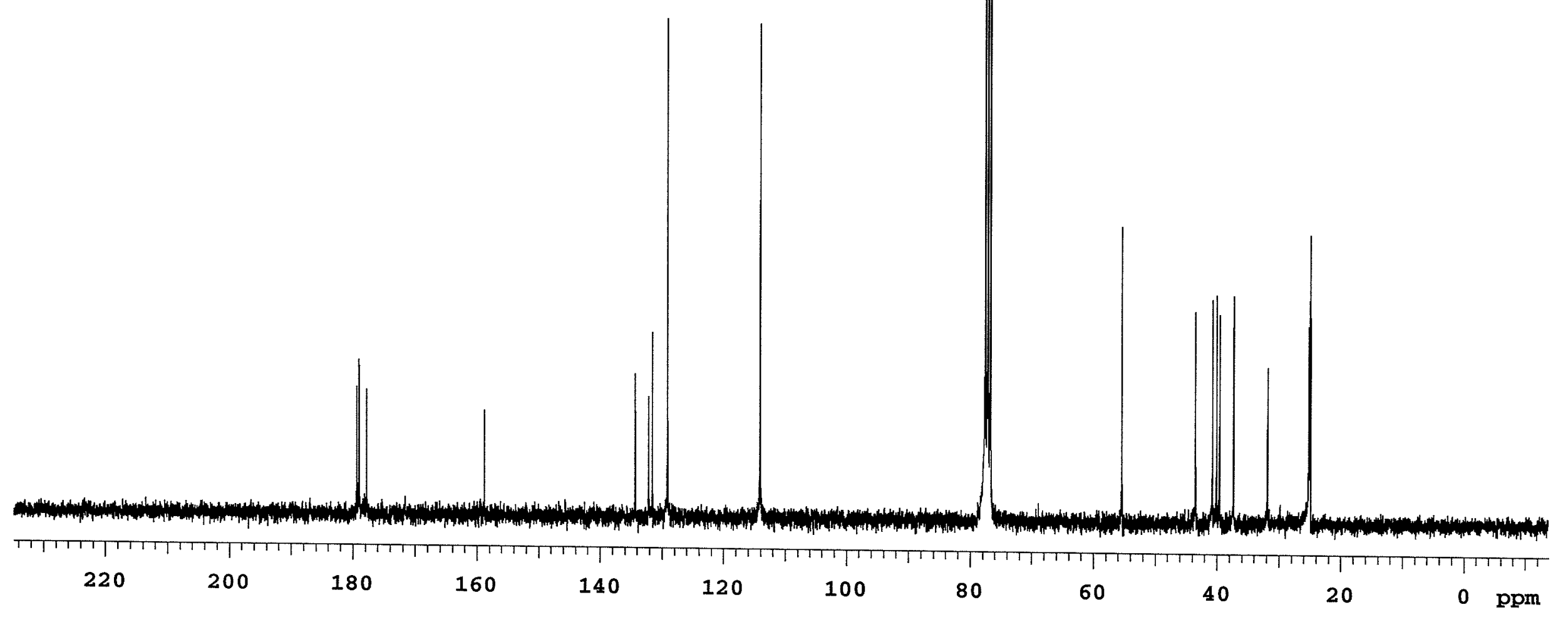




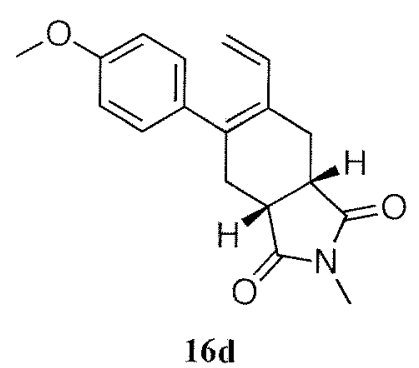

$500 \mathrm{MHz}, \mathrm{CDCl}_{3}$

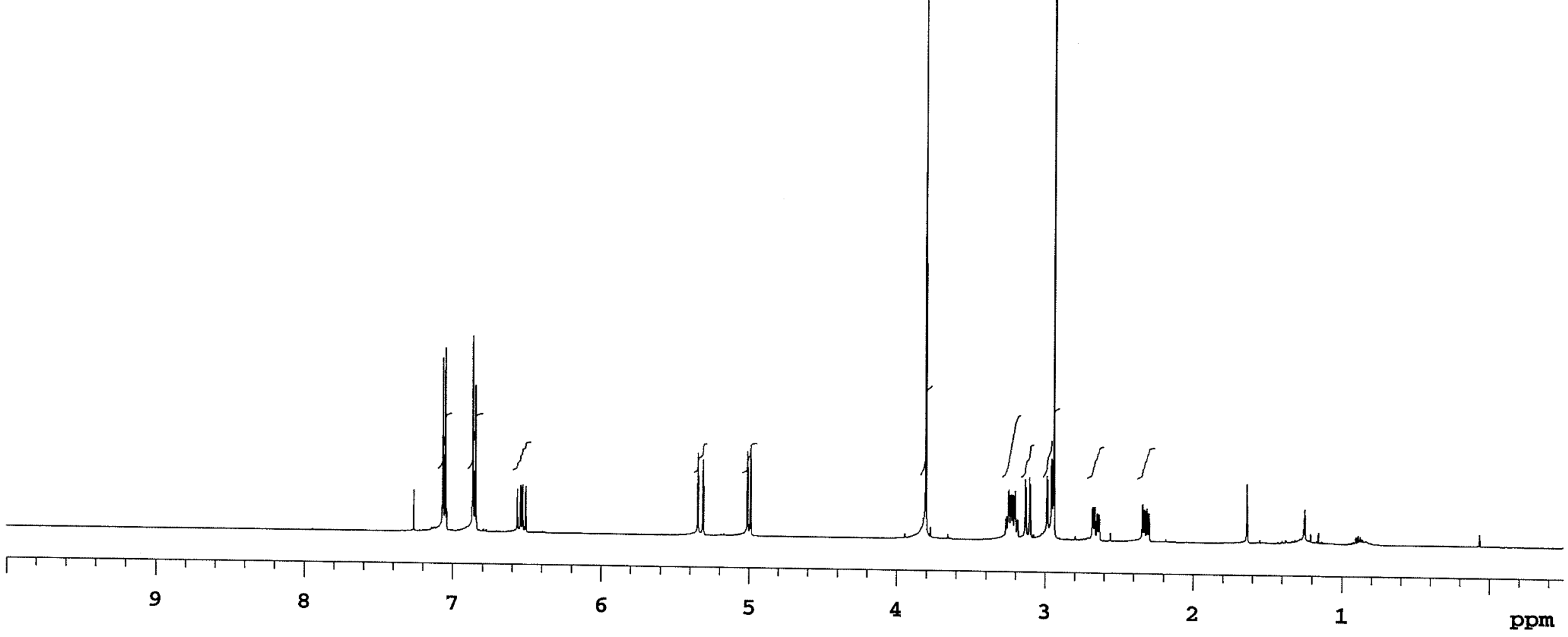




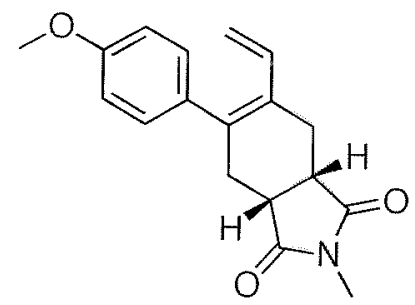

16d

$75 \mathrm{MHz}_{2} \mathrm{CDCl}_{3}$

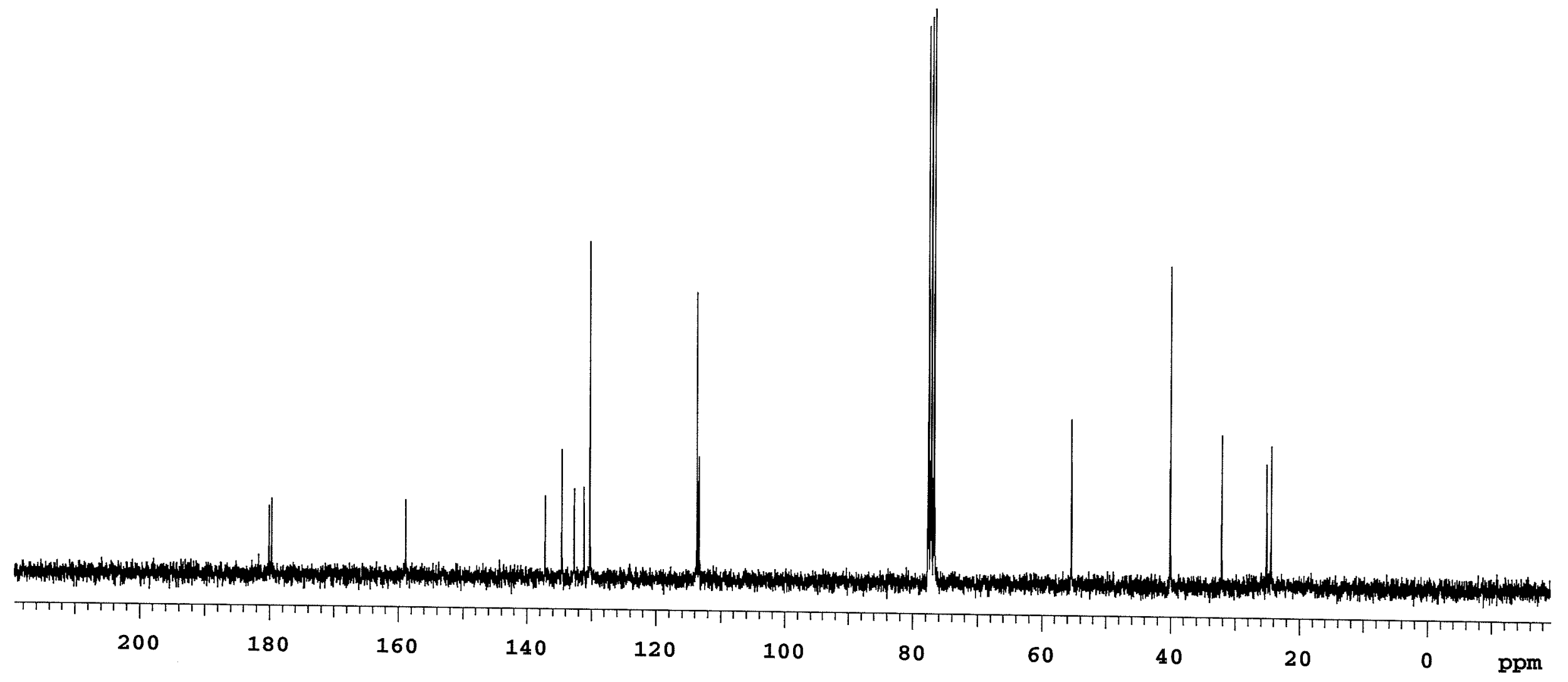




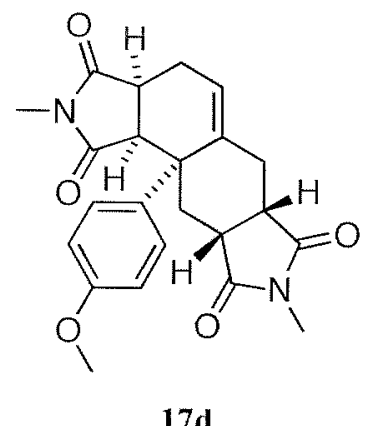

$600 \mathrm{MHz}_{3} \mathrm{CDCl}_{3}$

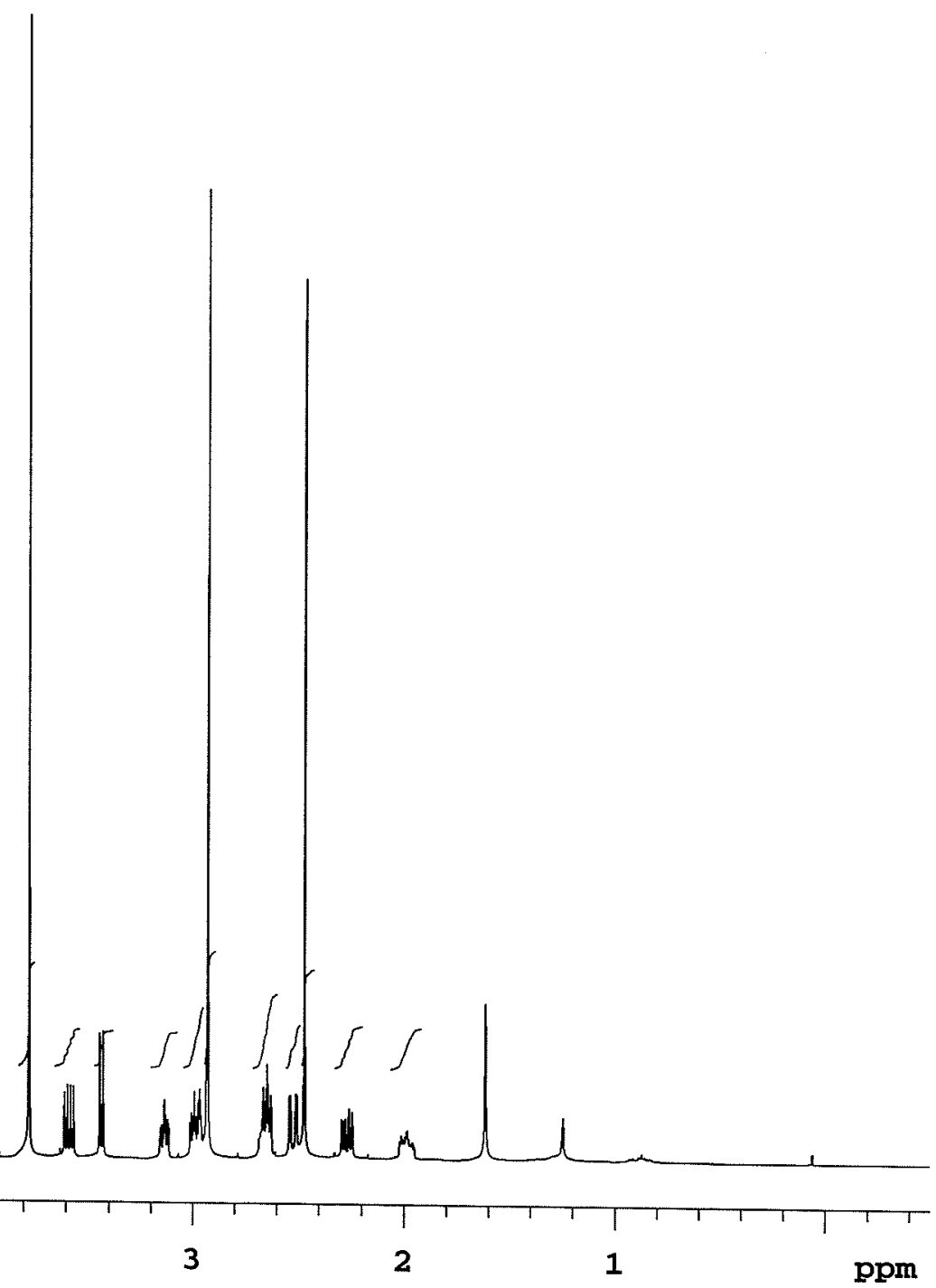




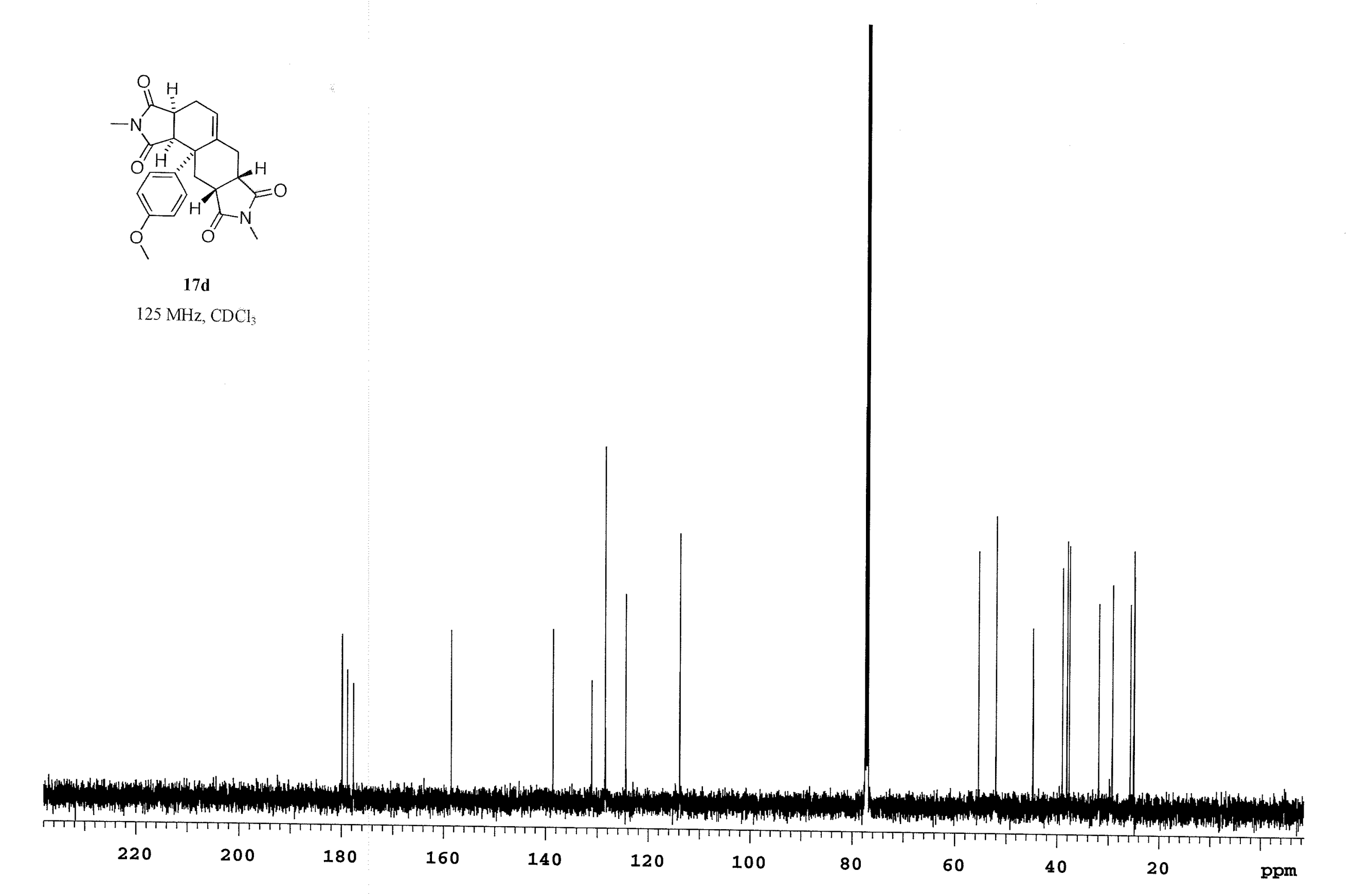




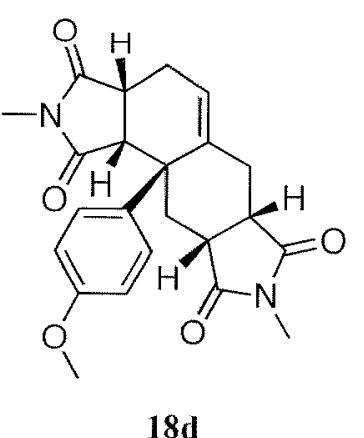

$800 \mathrm{MHz}, \mathrm{CDCl}_{3}$

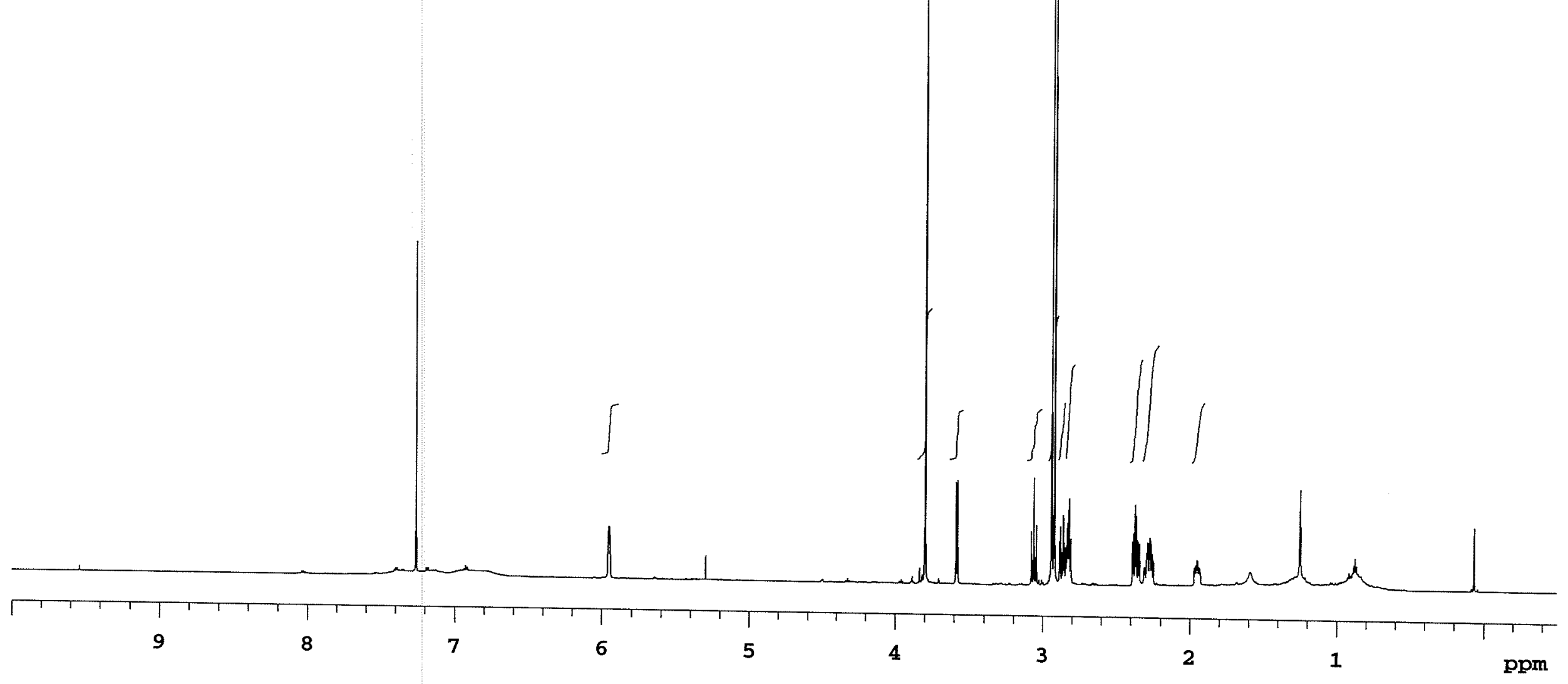




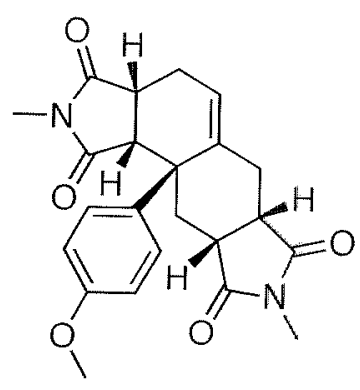

$18 d$

$75 \mathrm{MHz} \mathrm{CDCl}_{3}, 50{ }^{\circ} \mathrm{C}$

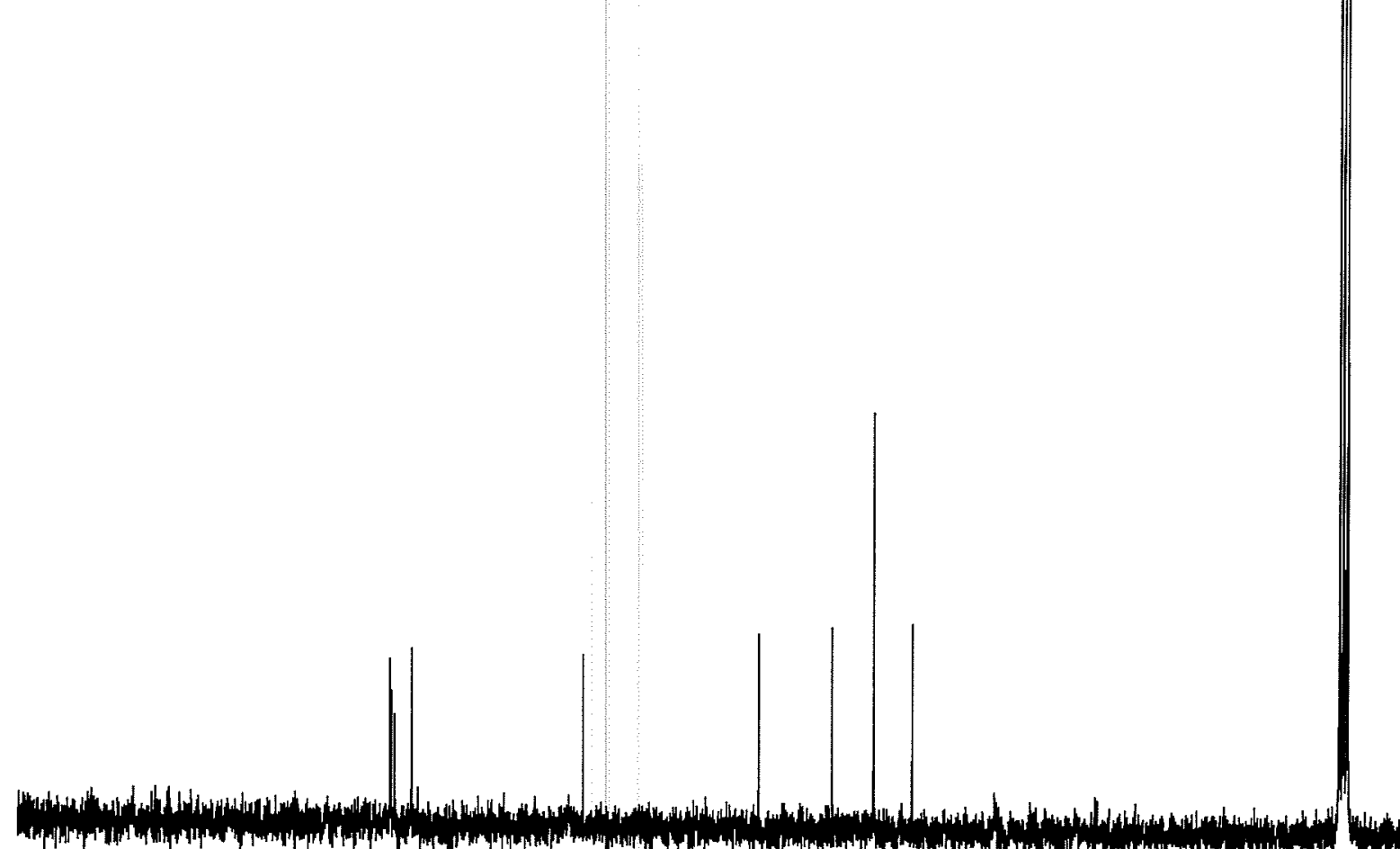

A r.

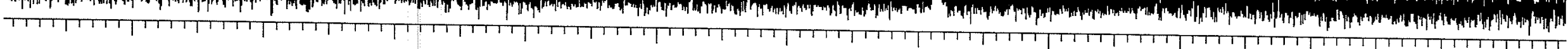

200

160

140

120

100

80

60

40

20

0

ppm 


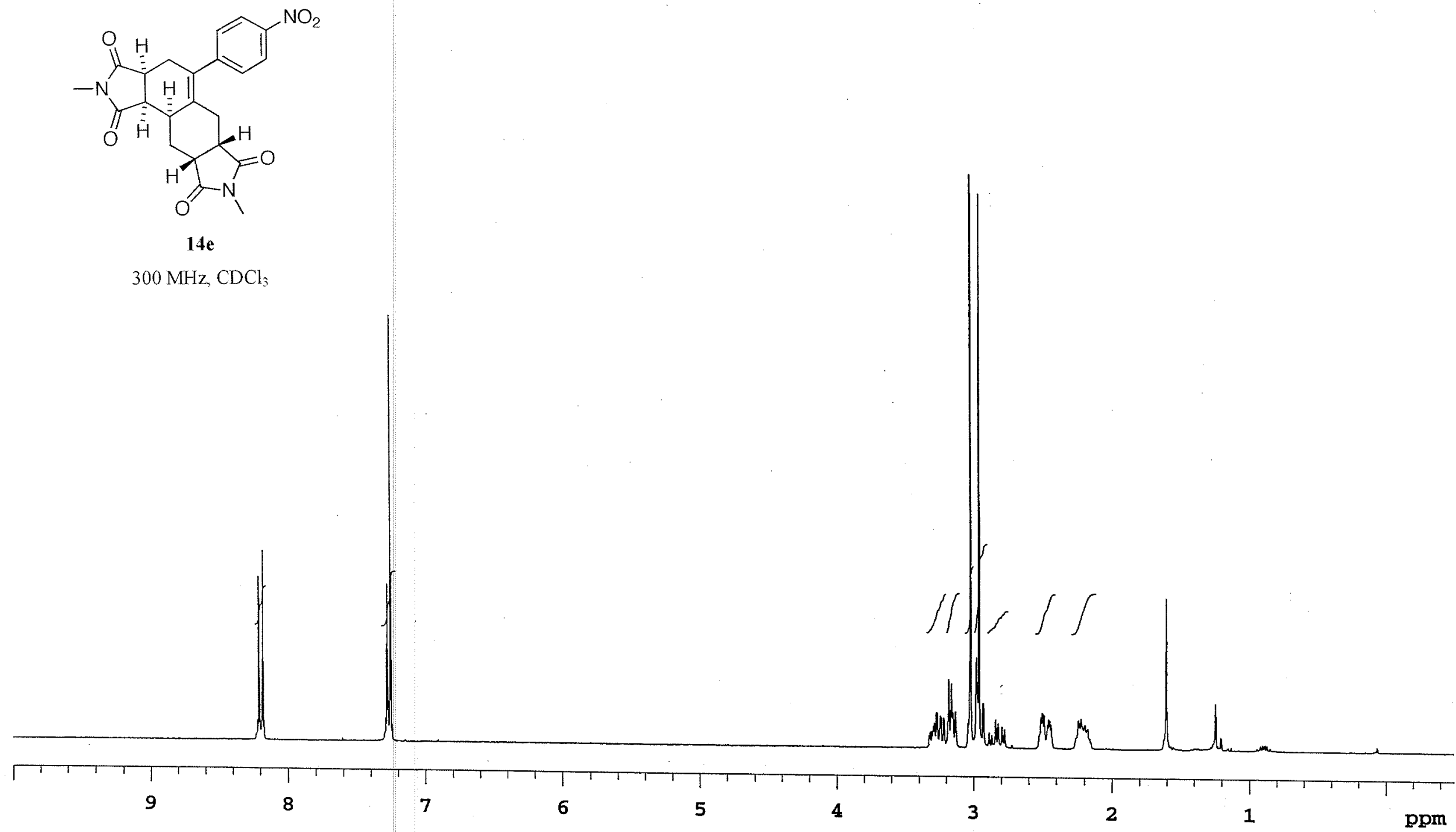




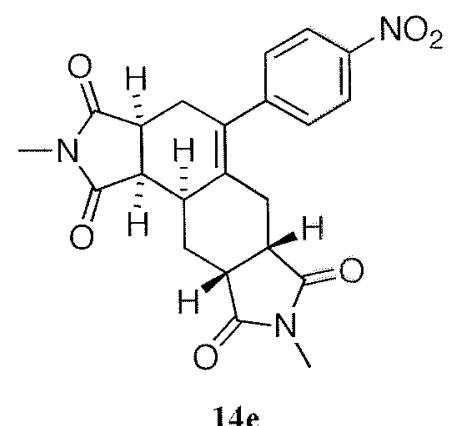

$75 \mathrm{MHz}, \mathrm{CDCl}_{3}$
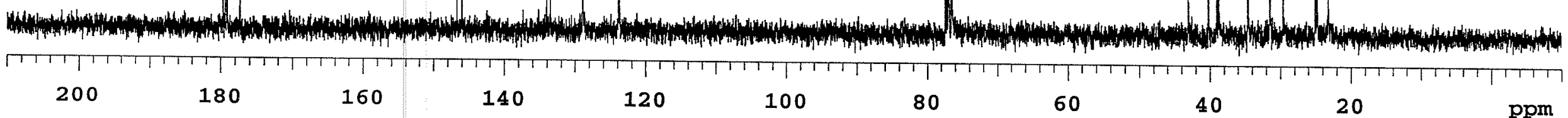


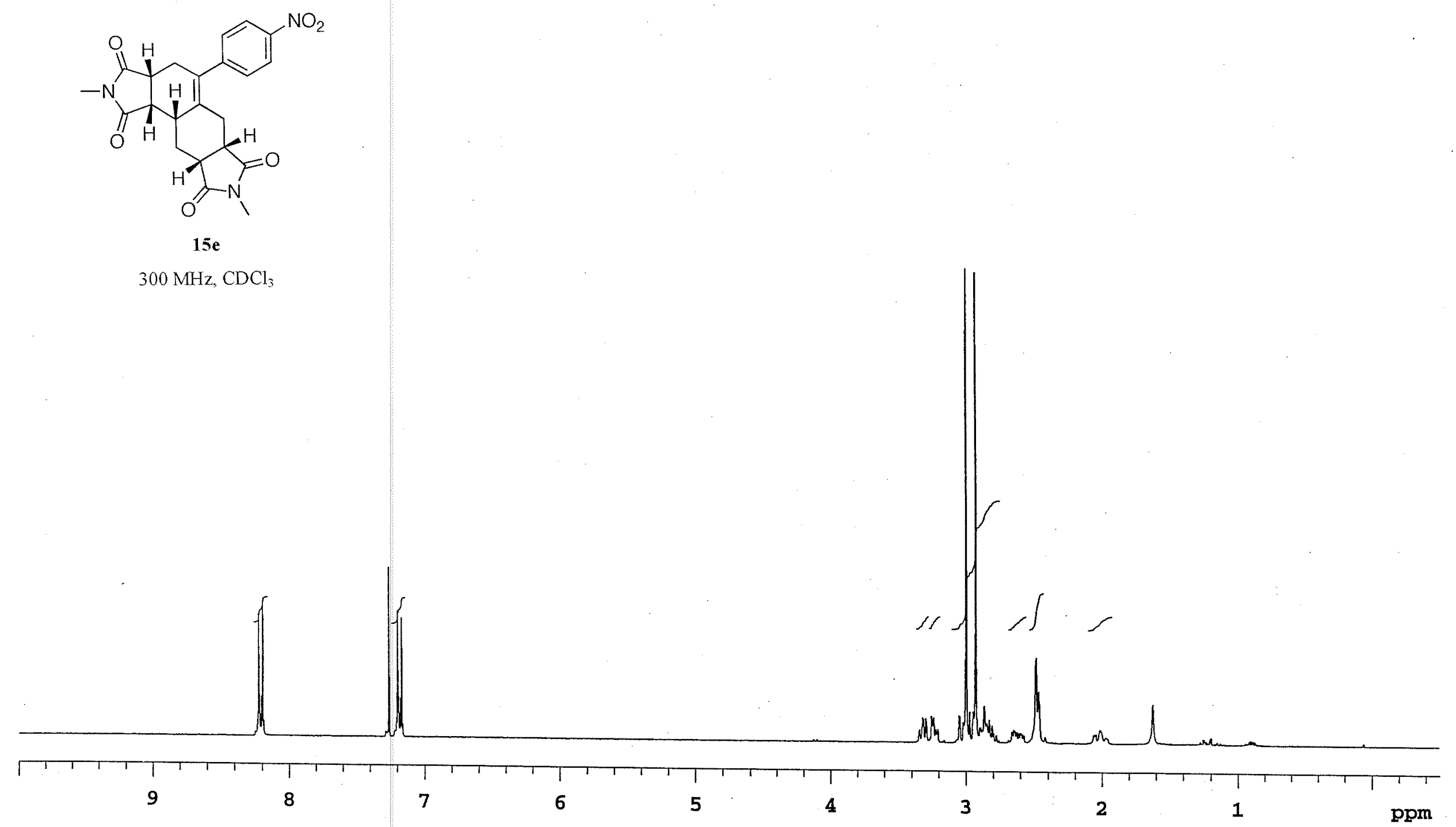




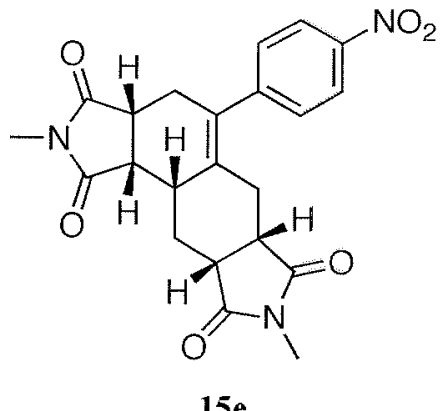

$15 \mathrm{e}$

$75 \mathrm{MHz}_{2} \mathrm{CDCl}_{3}$

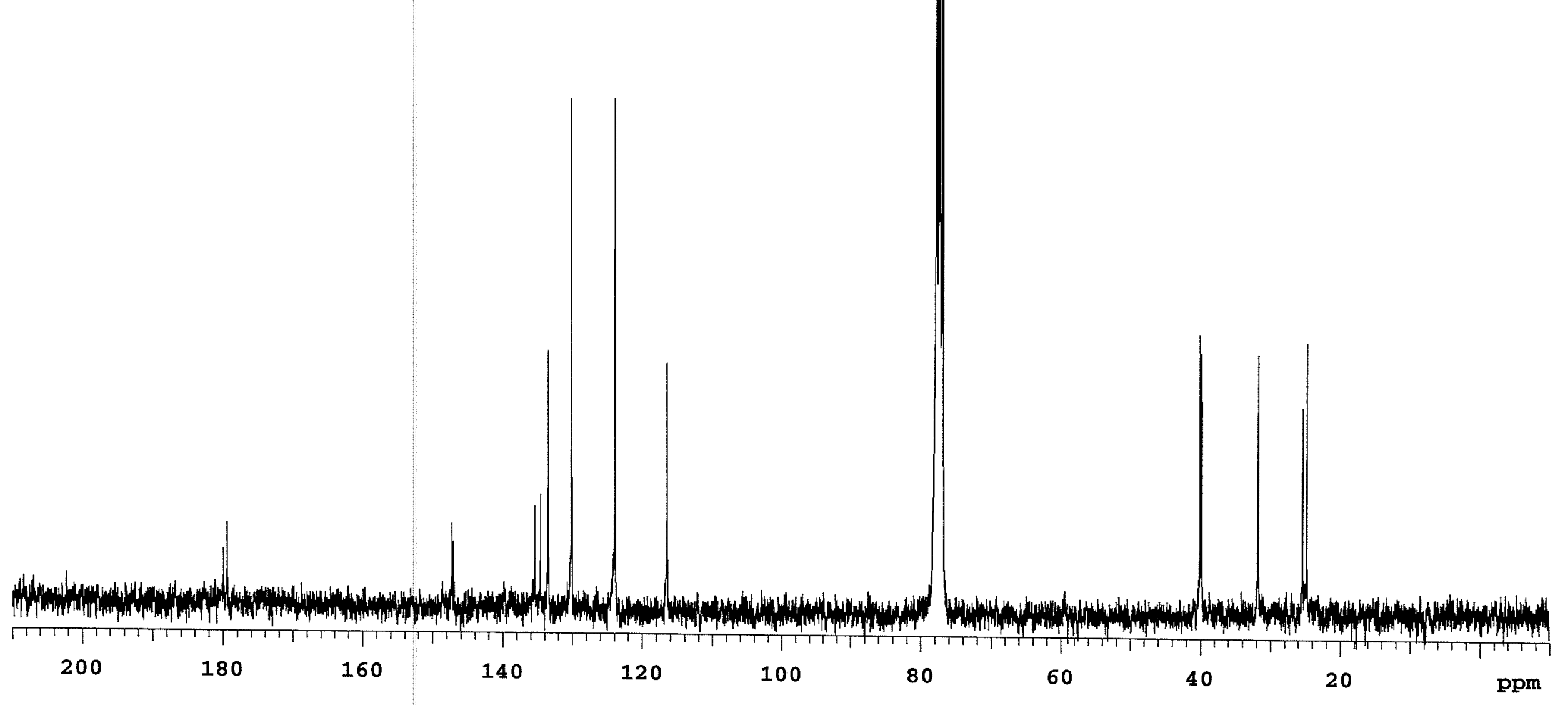




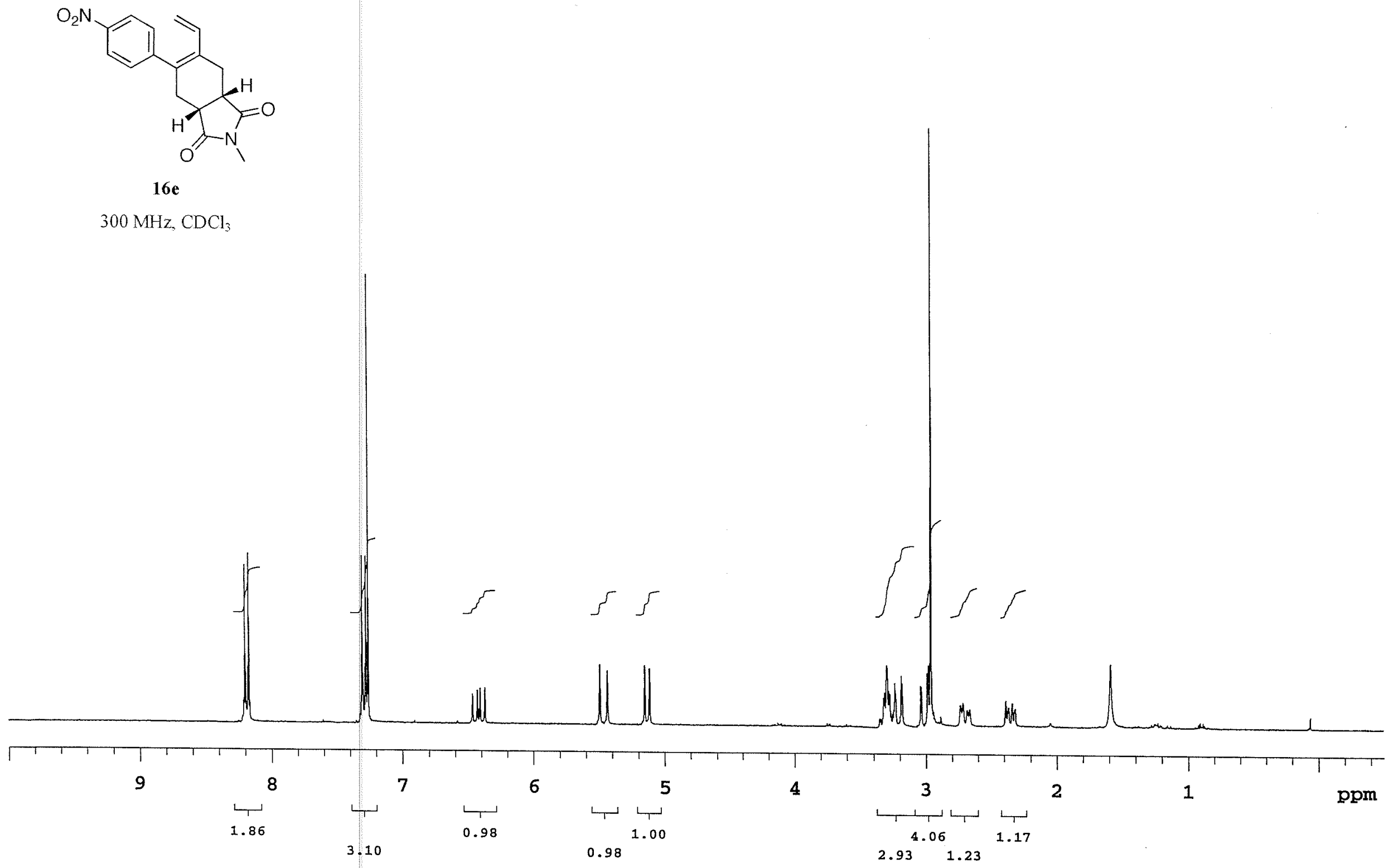




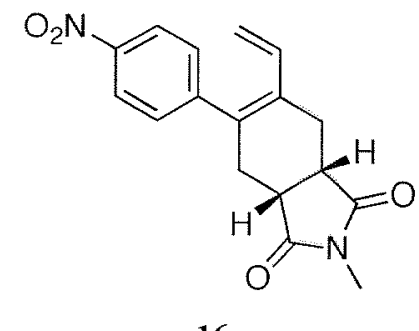

$16 \mathrm{e}$

$75 \mathrm{MHz} \mathrm{CDCl}_{3}$

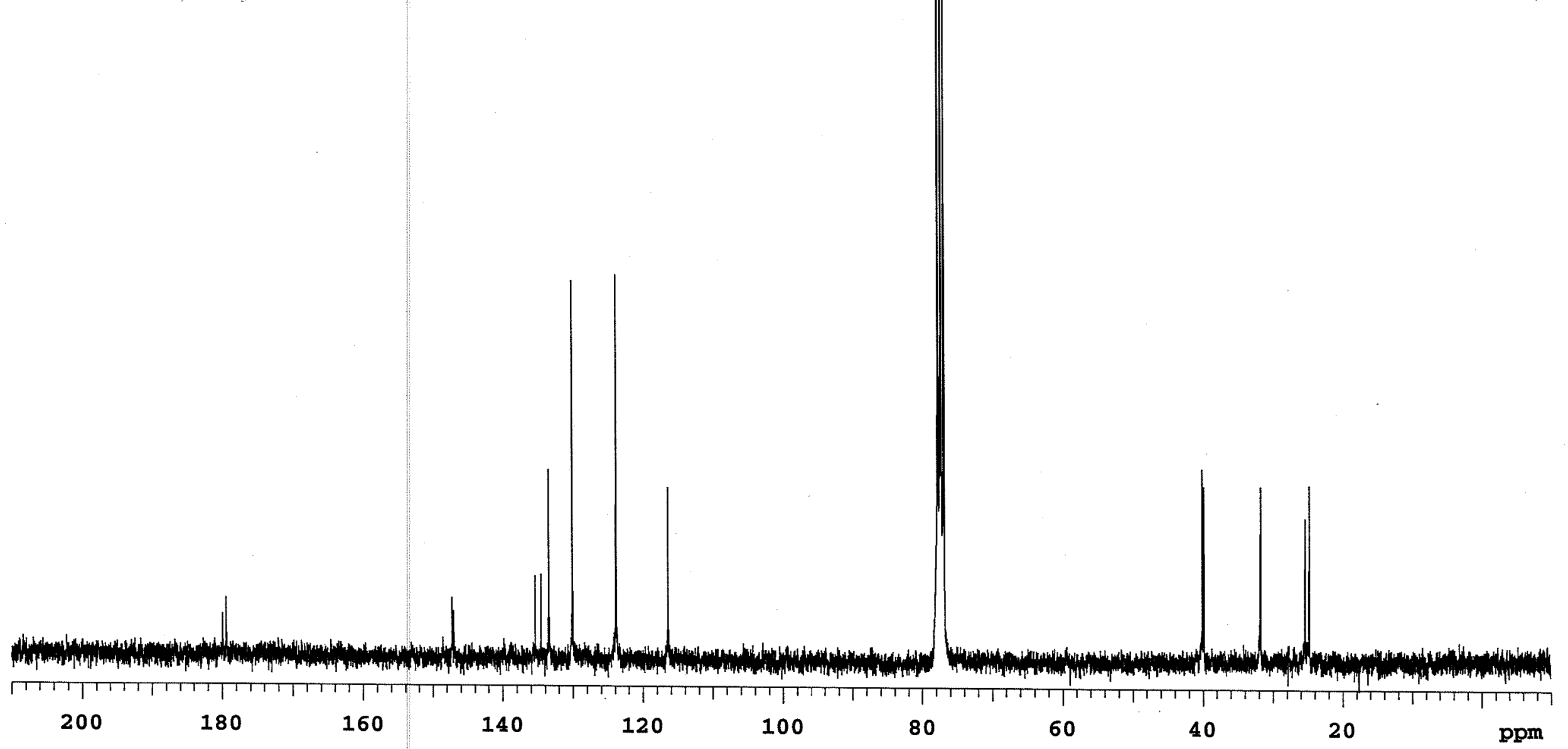




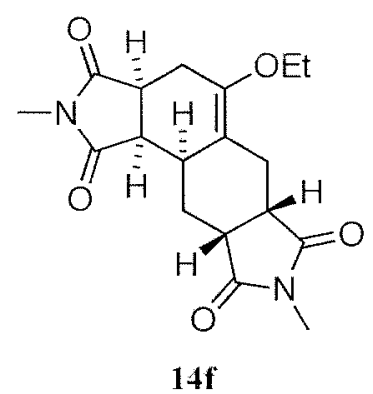

$300 \mathrm{MHz}, \mathrm{CDCl}_{3}$

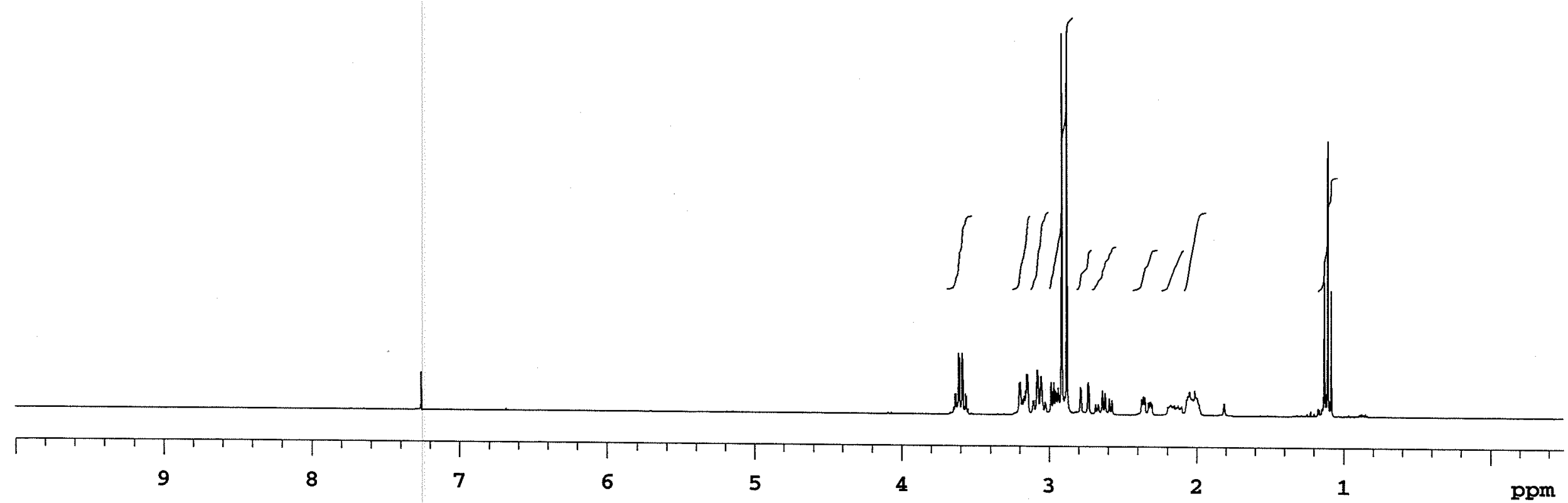




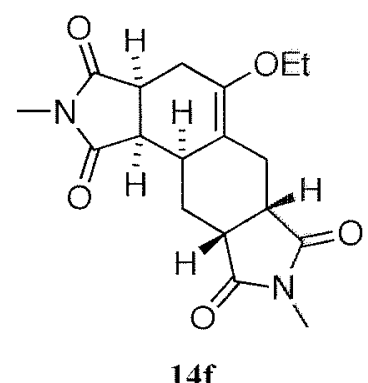

$75 \mathrm{MHz}, \mathrm{CDCl}_{3}$

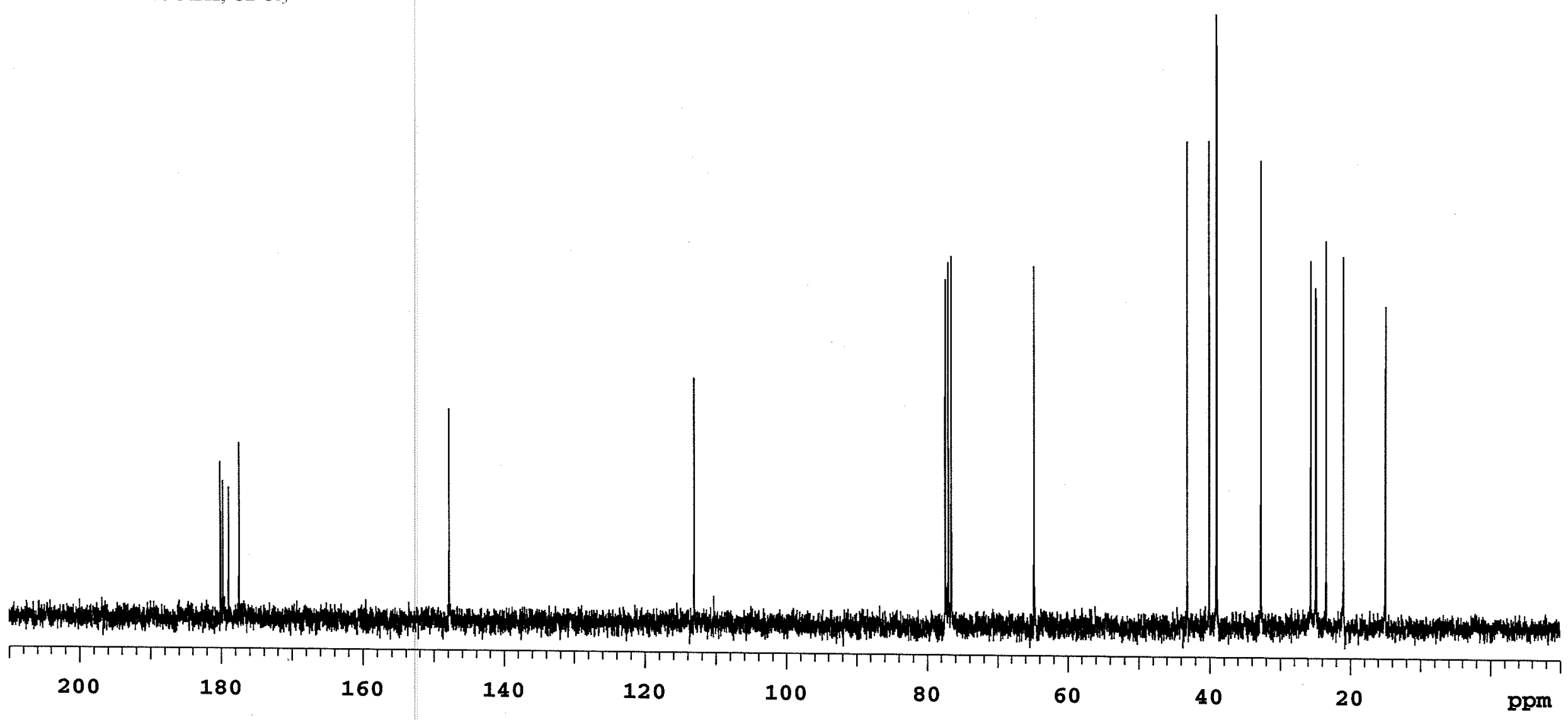




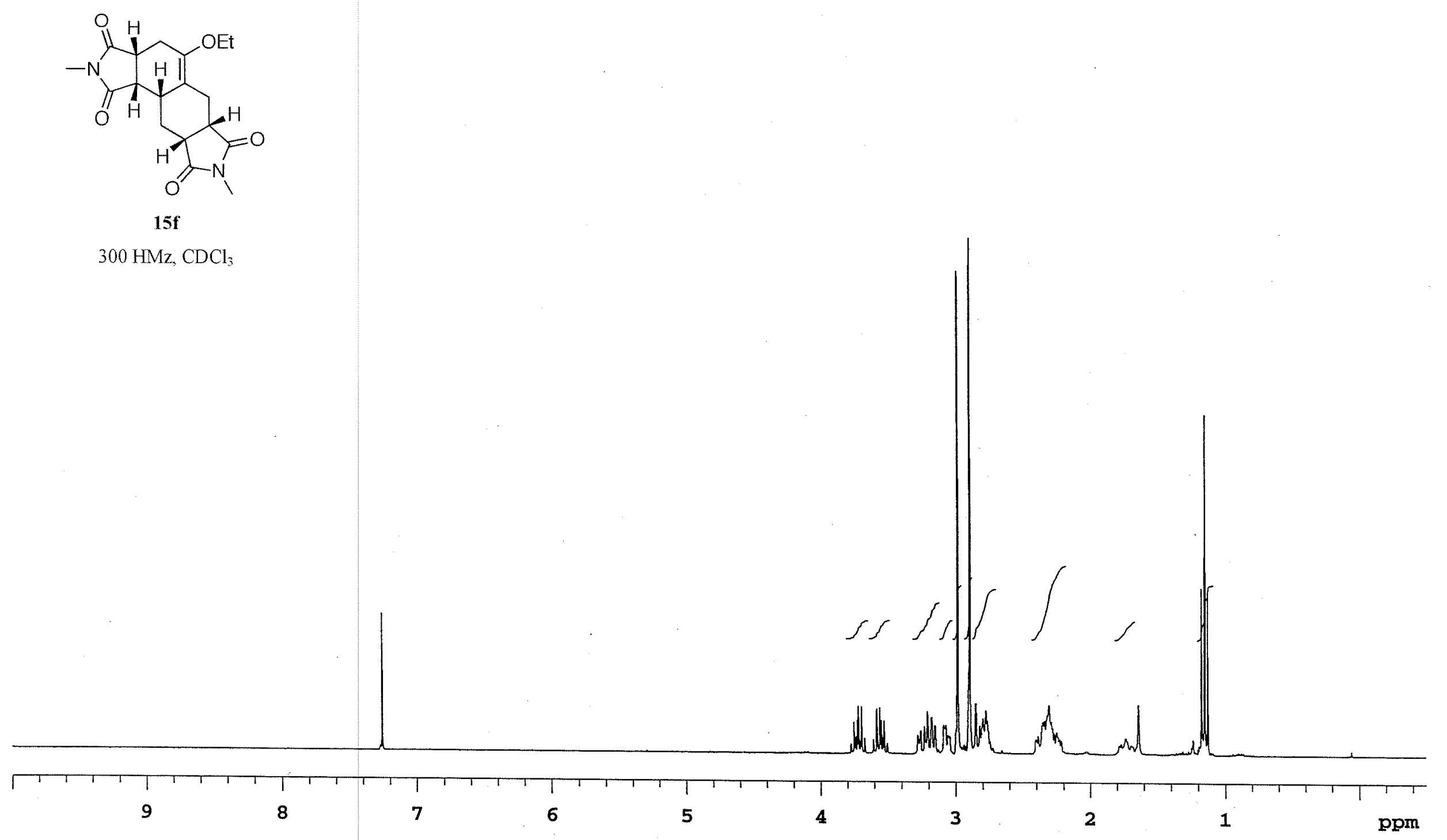




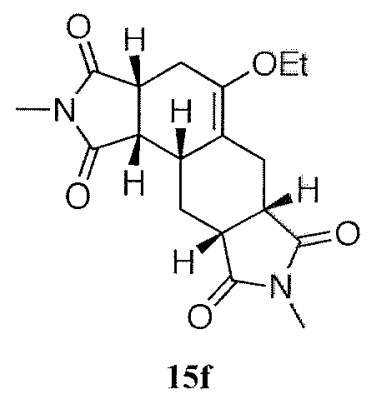

$125 \mathrm{HMz} \mathrm{CDCl}_{3}$

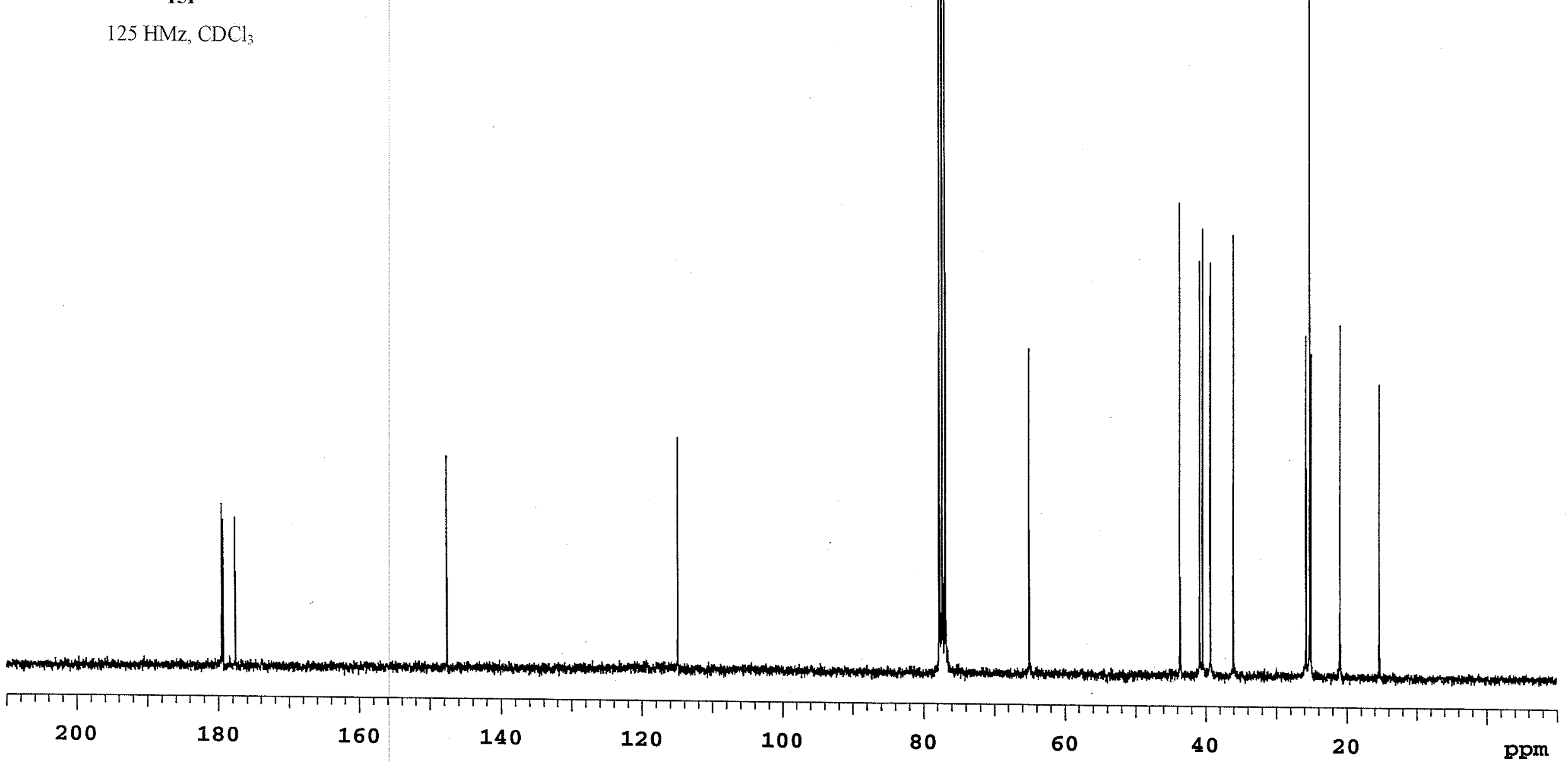

S106 


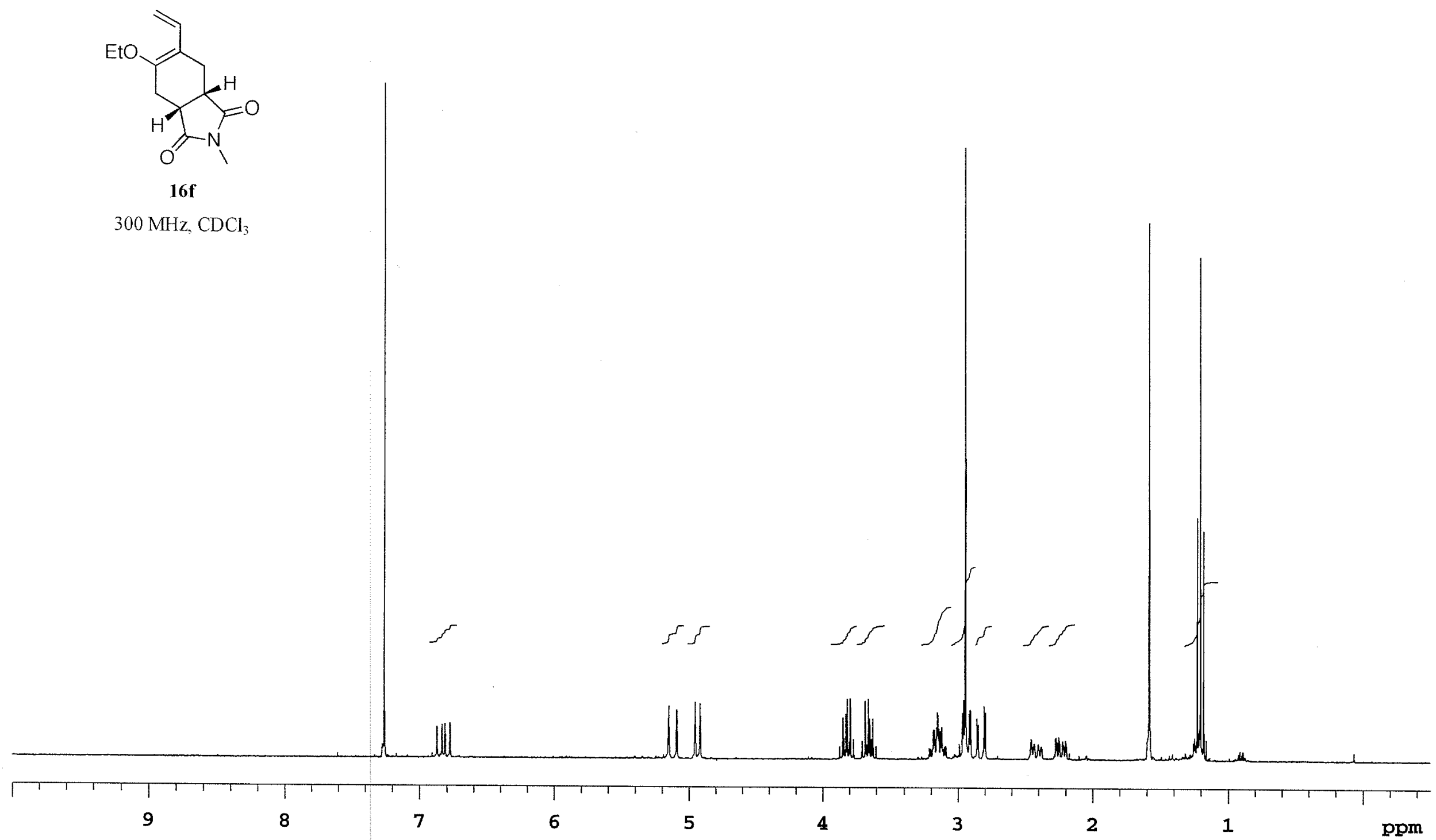




$$
{ }^{2}
$$




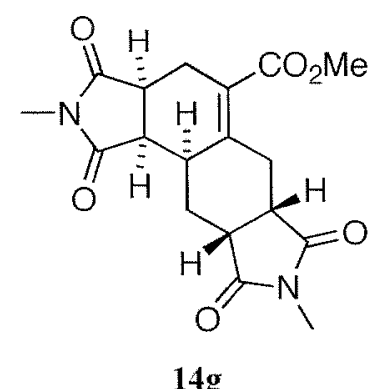

$300 \mathrm{MHz}_{2} \mathrm{CDCl}_{3}$

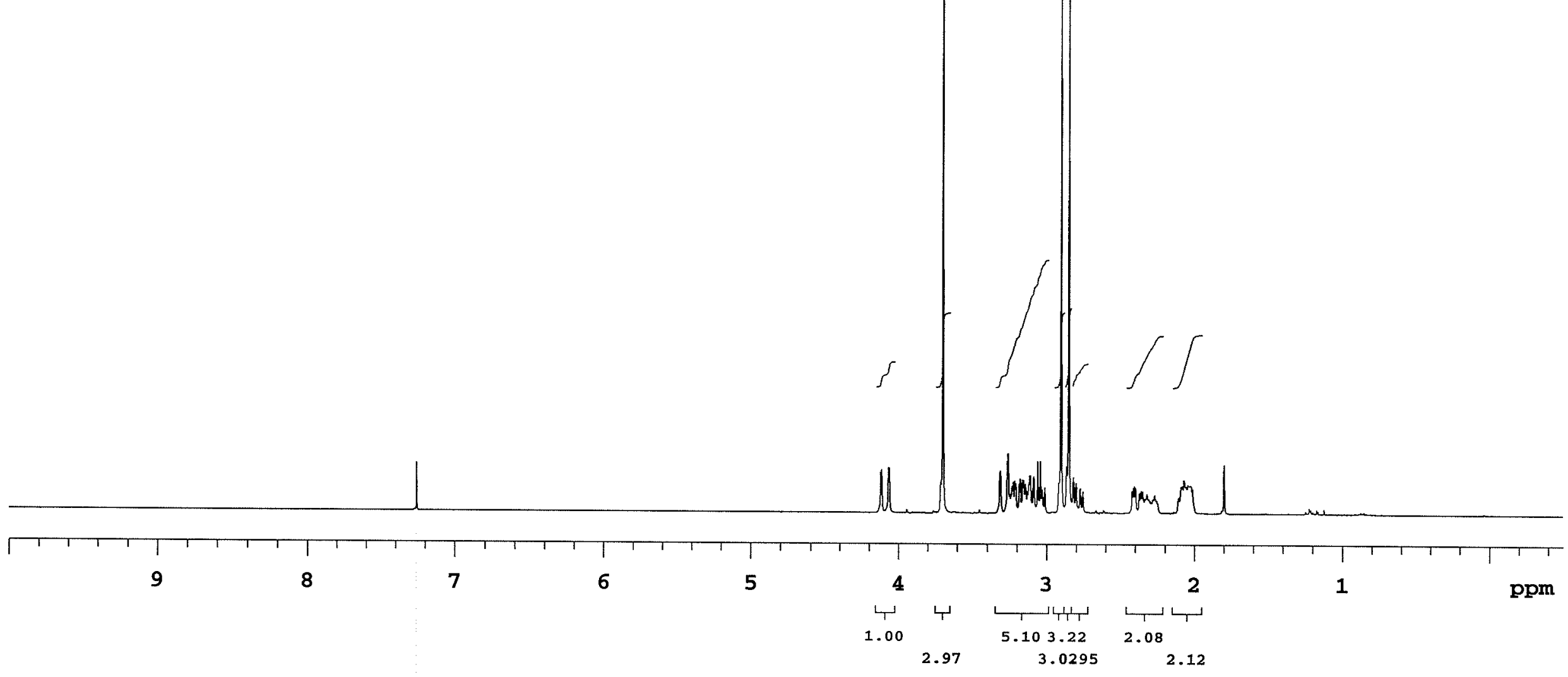




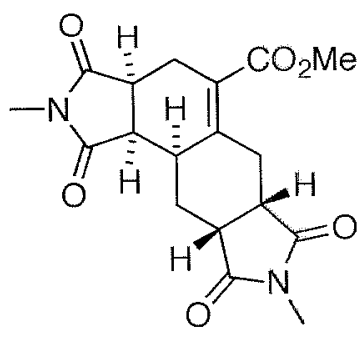

$14 \mathrm{~g}$

$75 \mathrm{MHz}, \mathrm{CDCl}_{3}$

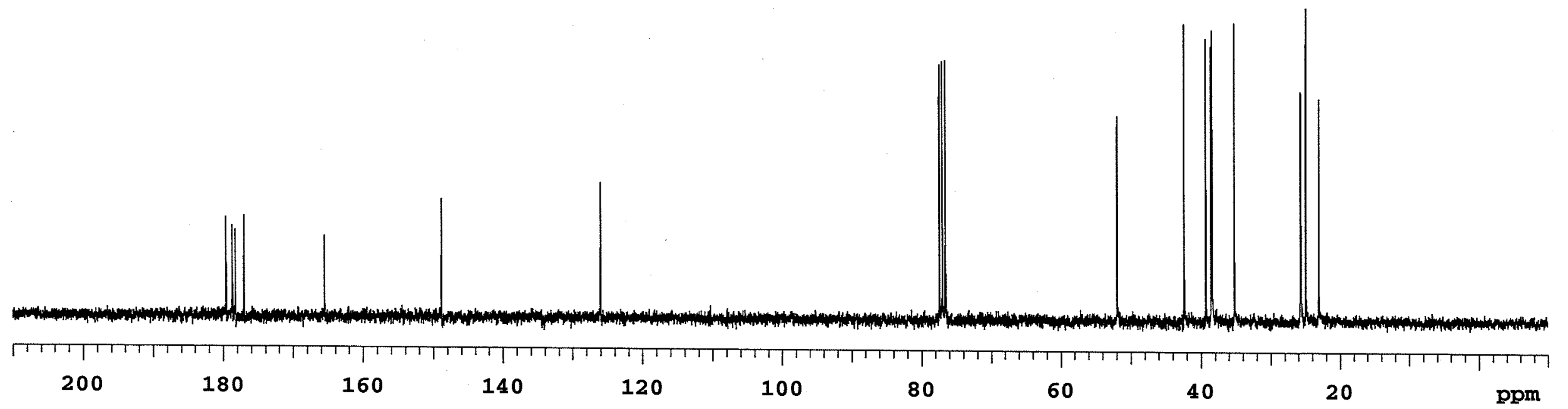




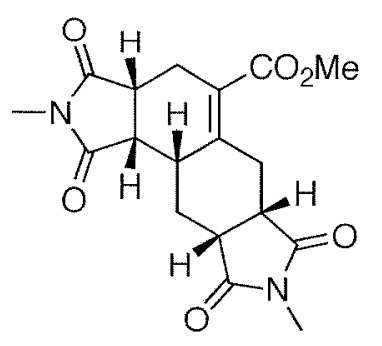

$15 \mathrm{~g}$

$800 \mathrm{MHz}_{2} \mathrm{CDCl}_{3}$

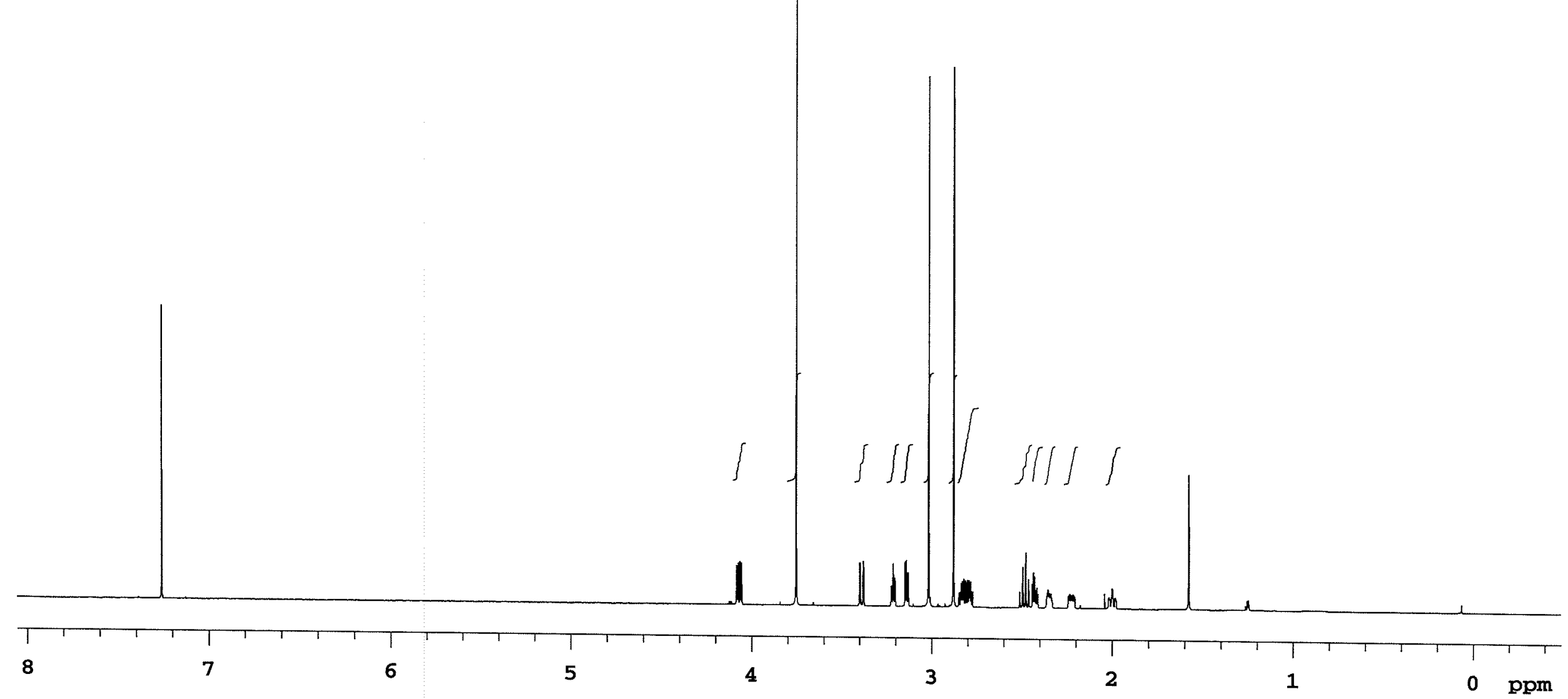




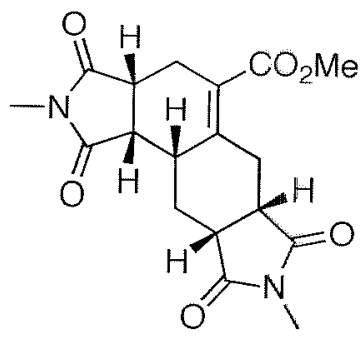

$15 \mathrm{~g}$

$75 \mathrm{MHz}_{2} \mathrm{CDCl}_{3}$

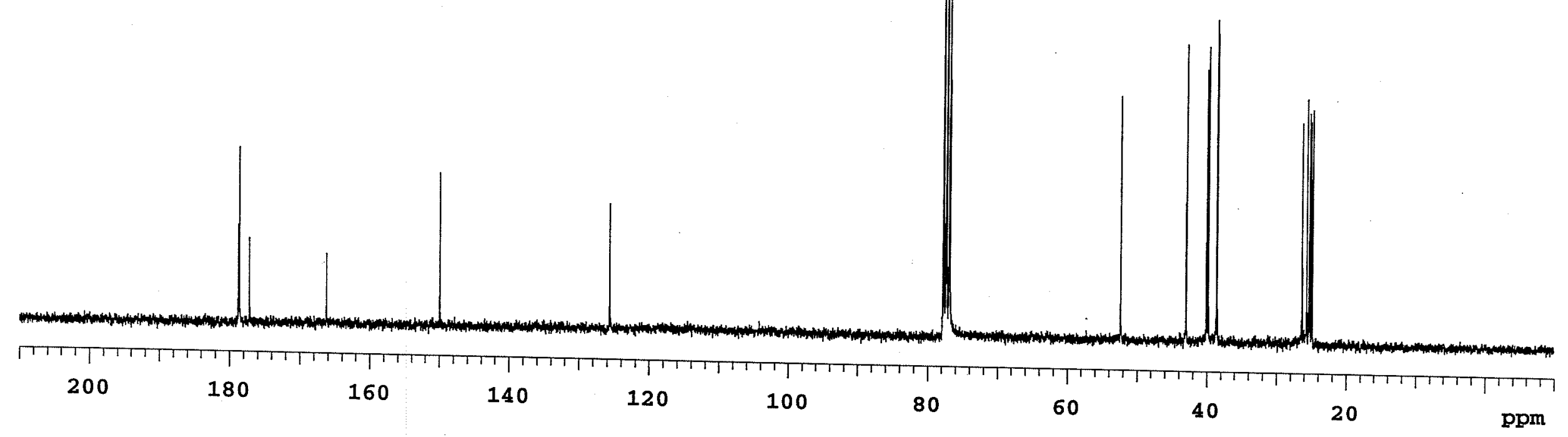




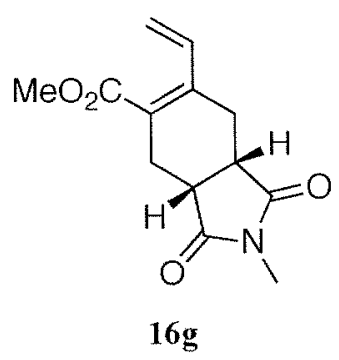

$300 \mathrm{MHz}_{2} \mathrm{CDCl}_{3}$

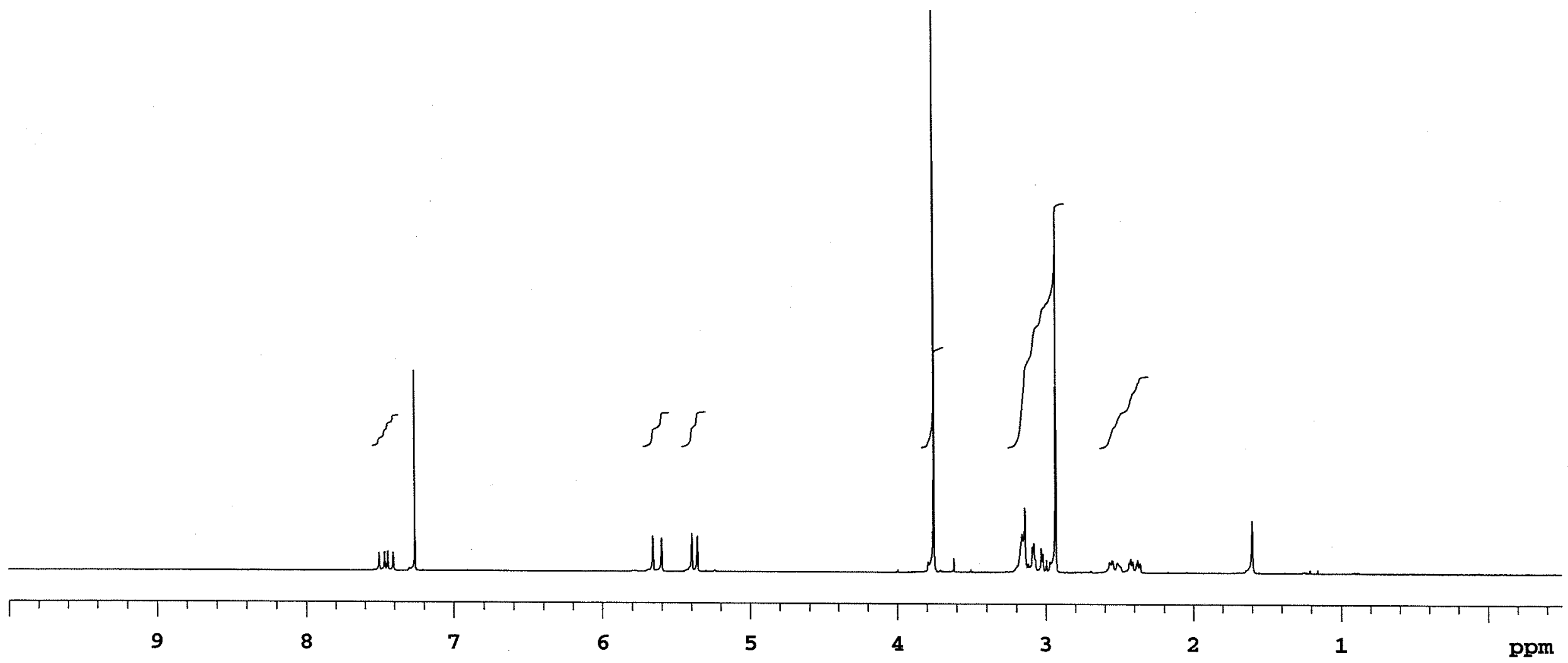

S113 
<smiles>C=CC1=C(C(C)=O)C[C@H]2C(=O)N(C)C(=O)[C@H]2C1</smiles>

$75 \mathrm{MHz}_{2} \mathrm{CDCl}_{3}$

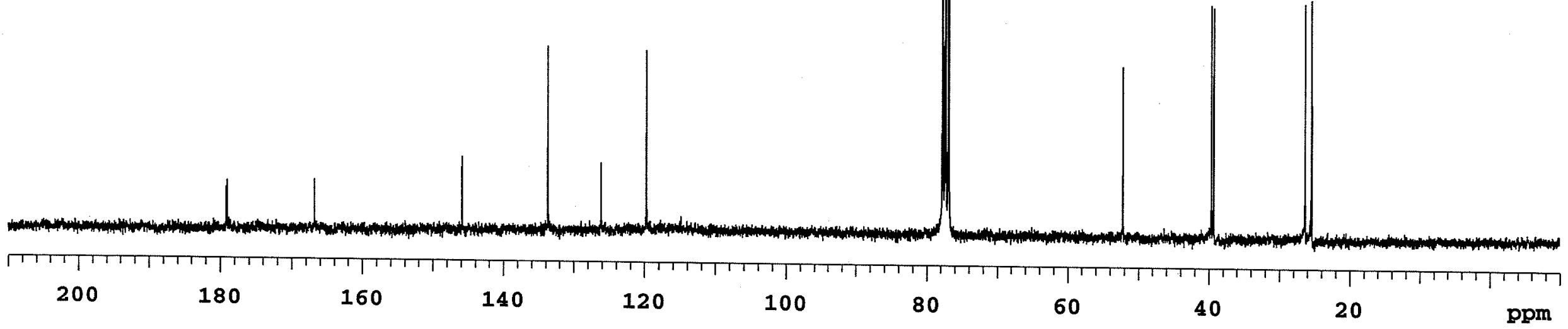

S114 


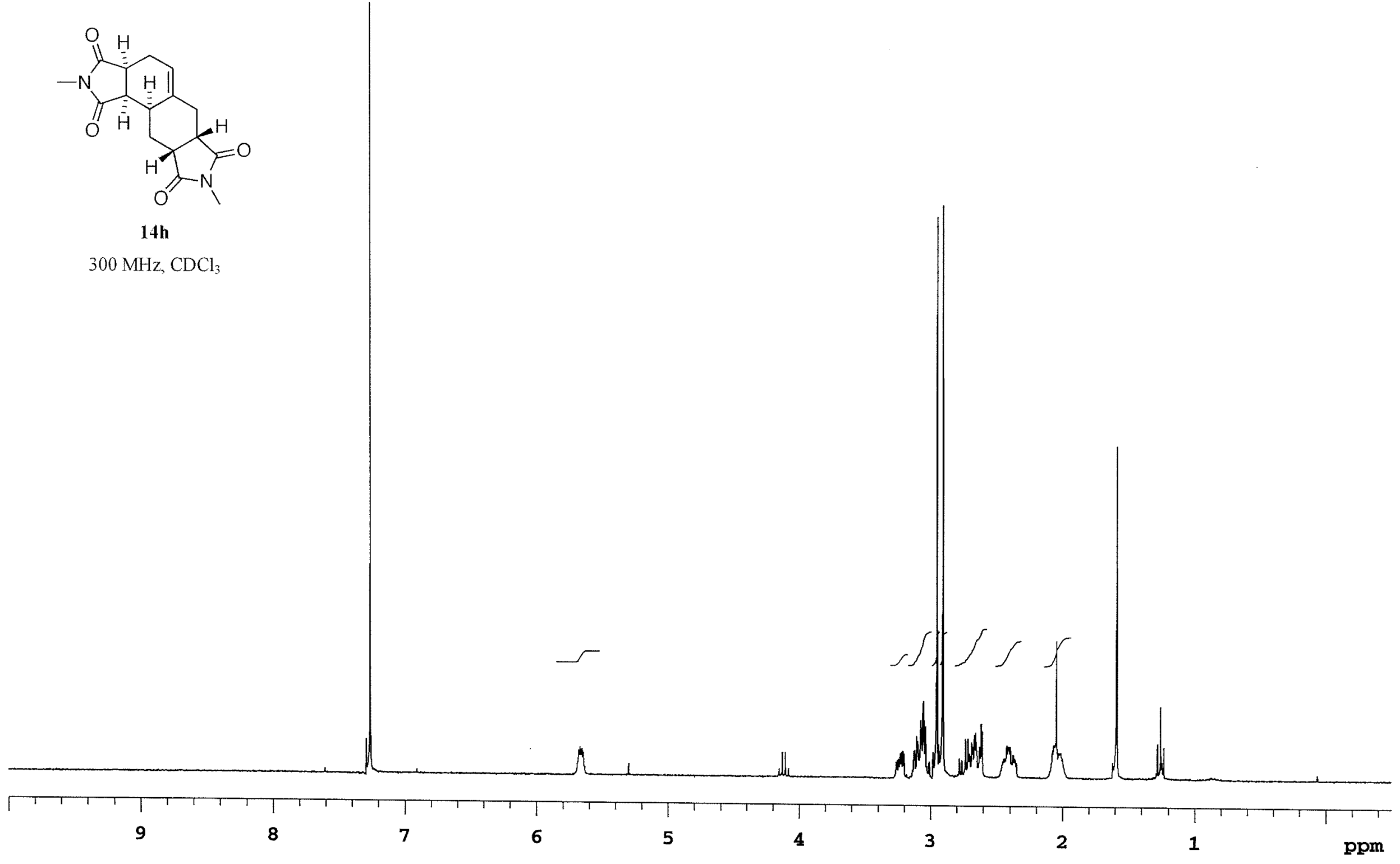




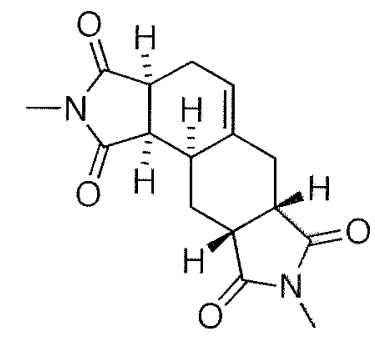

14h

$75 \mathrm{MHz}, \mathrm{CDCl}_{3}$

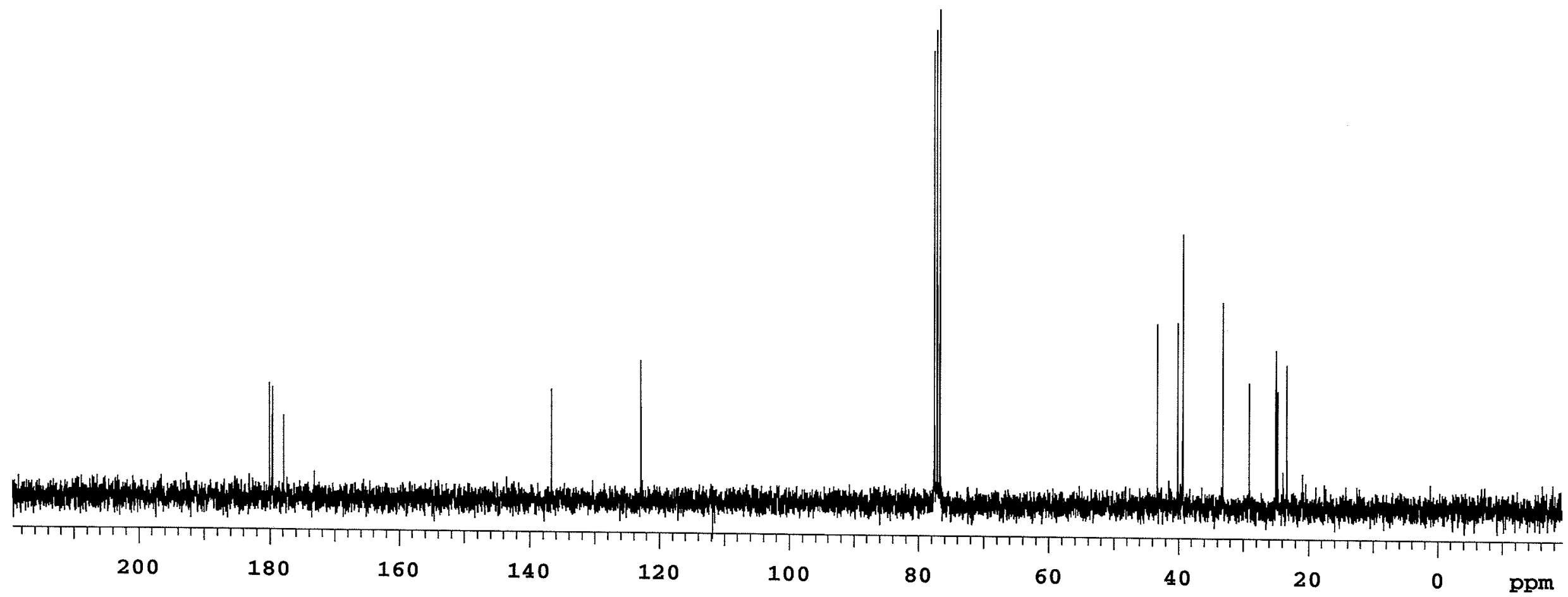




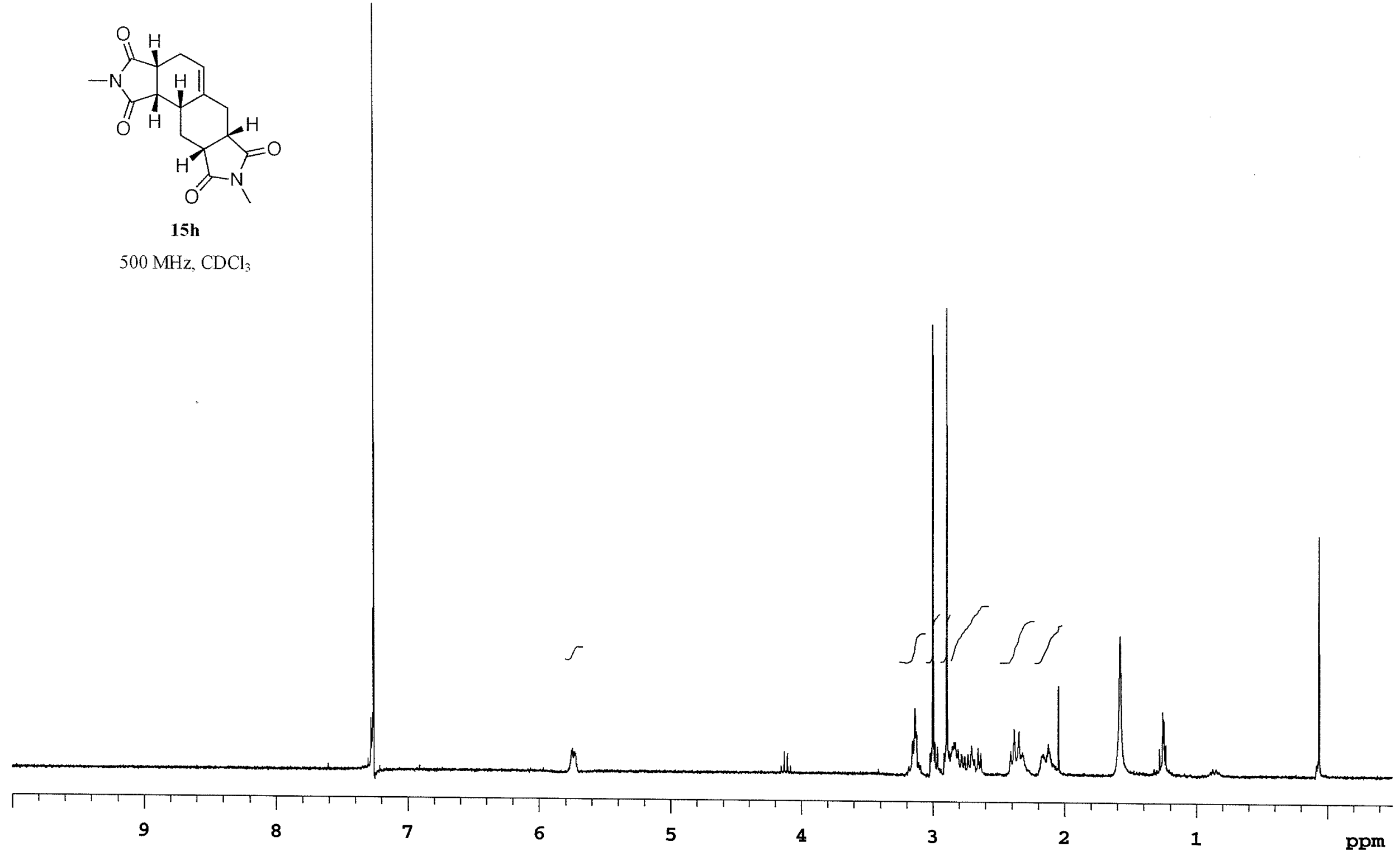




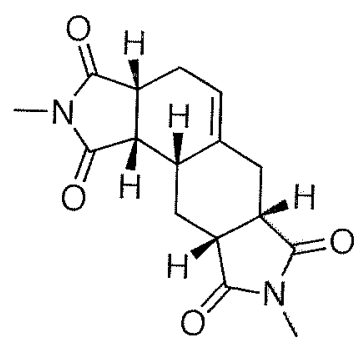

$15 \mathrm{~h}$

$125 \mathrm{MHz}_{2} \mathrm{CDCl}_{3}$

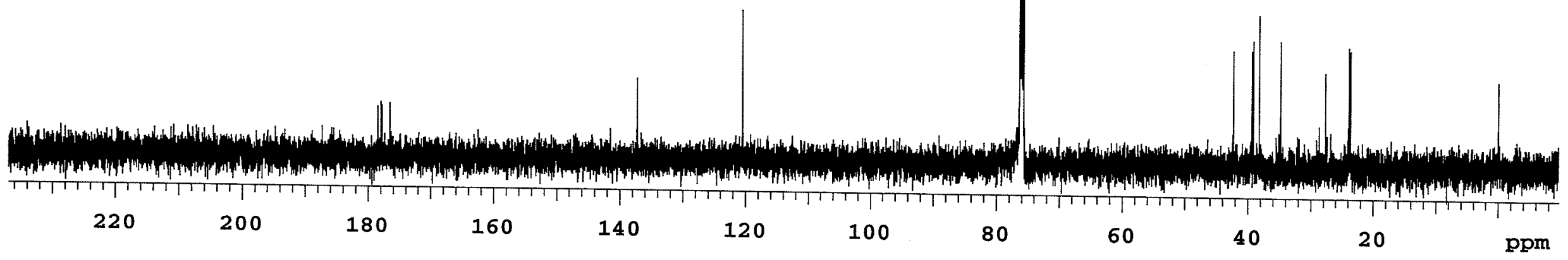

